\title{
Correlation of Site Variability from SASW and CPT Measurements
}

\author{
A Thesis \\ presented to
}

the Faculty of the Graduate School

at the University of Missouri

In Partial Fulfillment

of the Requirements of the Degree

Master of Science

By

JESSE F. MOYLES

Dr. Brent L. Rosenblad, P. E., Thesis Supervisor

MAY 2018 
The undersigned, appointed by the dean of the Graduate School, have examined the thesis entitled

\section{CORRELATION OF SITE VARIABILITY FROM SASW AND CPT MEASUREMENTS}

Presented by Jesse F. Moyles

A candidate for the degree of Master of Science

And hereby certify that in their opinion it is worthy of acceptance

Dr. Brent L. Rosenblad, P.E., Chair

Dr. John J. Bowders, P.E.

Dr. Allen L. Thompson, P.E. 


\section{ACKNOWLEDGEMENTS}

There are several people, and a few institutions, to whom I owe a great debt of gratitude. I'll start by thanking the entire University of Missouri Geotechnical Engineering faculty and staff for facilitating a fantastic graduate program at MU. I am especially thankful to my faculty advisors, Dr. Brent Rosenblad and Dr. John Bowders, who were involved with this project from start to finish and are the source of much professional guidance and personal inspiration. I very much appreciate that they let me take this thesis my own direction, though they well knew it was the wrong one at times. I would also like to thank Dr. J. Erik Loehr and Dr. Ahmed Abu El-Ela for taking the time to examine, discuss, and make meaningful suggestions regarding this project along the way. Additionally, I must thank my fellow students, Mohammed Khan, Nathaniel Dummerth, Hashim Al-Sumaiday, Ghaith Al-Sharify, and Nimer (The Hammer) Alselami who not only helped me with this project, but with all of my studies at MU.

None of this research would have been possible without the generosity of Ameren Missouri and Reitz \& Jens, Inc. I am especially thankful to Mike Wagstaff, of Ameren Missouri, and Jeff Fouse, of Reitz \& Jens, Inc., for coordinating access to the UWL site and associated CPT data. I must also thank my boss, Captain Kevin King (USN), for granting me the flexibility to complete this thesis while working full time.

On a more personal note, I am incredibly grateful for the support of my wonderful wife, Lauren, without whom I could never have completed this project. Her time and energy spent facilitating my work greatly outweighs what is reflected in the text. Finally, and most importantly, I would like to thank God for bringing together all things that made this work possible. 


\section{TABLE OF CONTENTS}

ACKNOWLEDGEMENTS..................................................ii

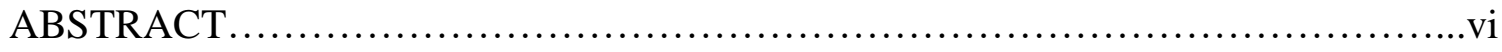

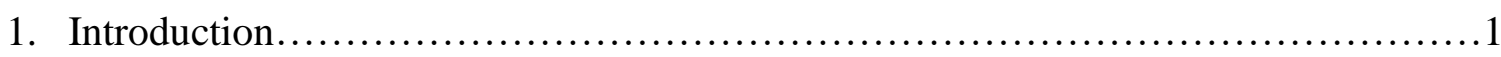

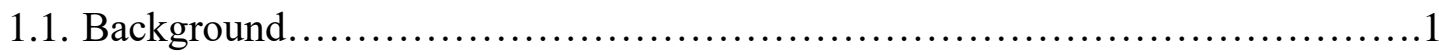

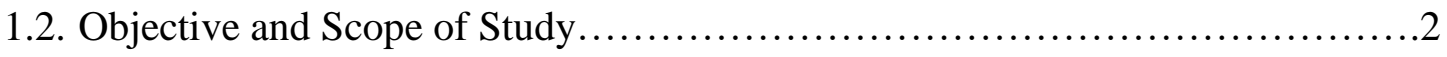

1.3. Thesis Organization................................................. 3

2. Literature Review.....................................................4

2.1. Overview.......................................................4

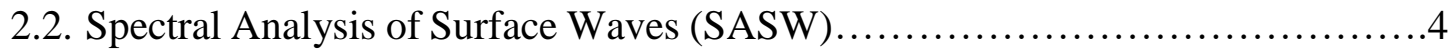

2.2.1. SASW \& Site Characterization....................................6

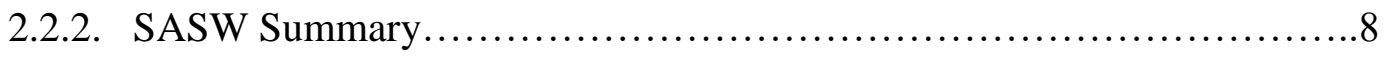

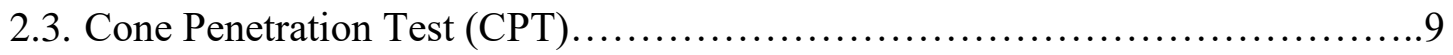

2.4. Boring and Sampling in Site Characterization Practice.......................11

2.5. Summary........................................................ 14

3. Site Description...................................................... 15

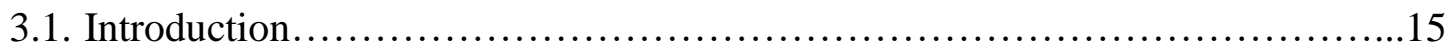

3.2. Labadie Unified Waste Landfill (UWL) Site............................ 15

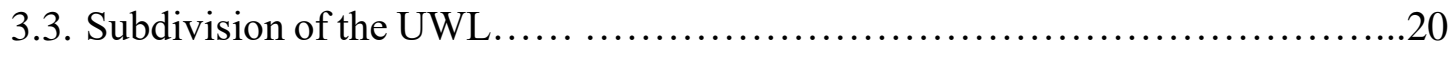

4. Methods............................................................... 22

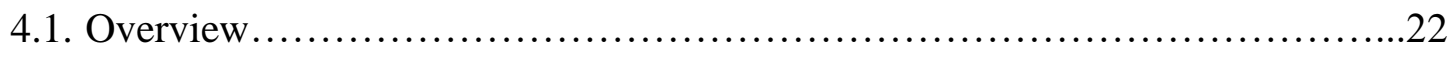

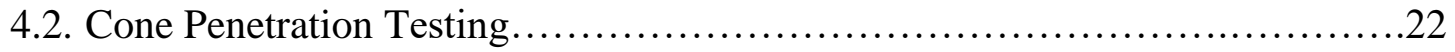




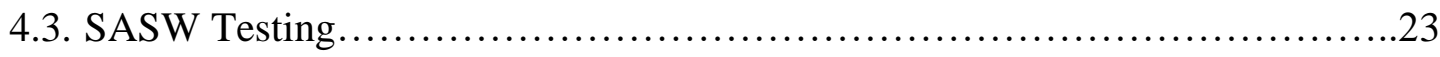

4.3.1. Equipment...................................................... 24

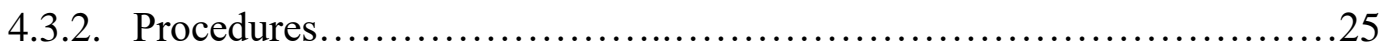

4.4. SASW Data Reduction and Dispersion Curve Fitting.........................27

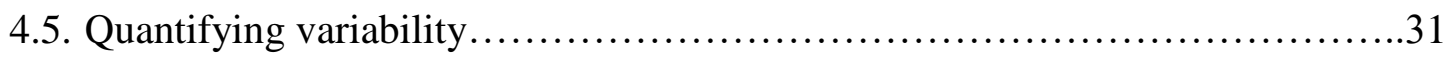

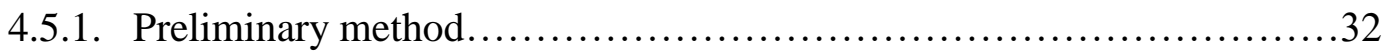

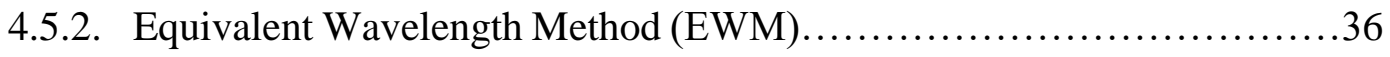

4.5.3. EWM Example Calculation...................................40

5. Results........................................................... 45

5.1. Overview ........................................................ 45

5.2. CPT Measurements and Initial Comparison.............................45

5.2.1. Verification of Selected CPT Soundings.............................51

5.2.2. Preliminary Variability Analysis of CPT Data........................53

5.3. SASW Dispersion Curves.......................................... 54

5.3.1. Verification of Selected SASW Tests..............................59

5.4. Preliminary Variability Correlation.....................................62

5.5. Equivalent Wavelength Method.......................................63

5.5.1. EWM Variability Correlation ....................................66

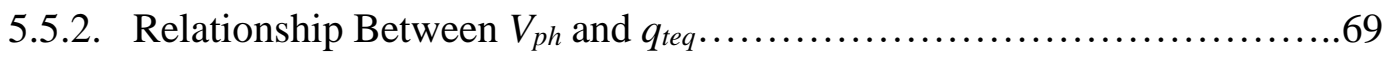

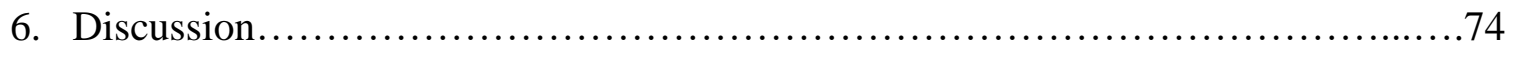

6.1. Overview........................................................ 74

6.2. Challenges in Comparing Variability of SASW and CPT Data...................74

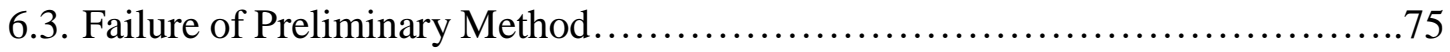


6.4. Variability Correlation with the EWM.

6.4.1. Exclusion of Point $147 \mathrm{~A}$ and $\lambda \leq 5 \mathrm{ft} \ldots \ldots \ldots \ldots \ldots \ldots \ldots \ldots \ldots \ldots \ldots$

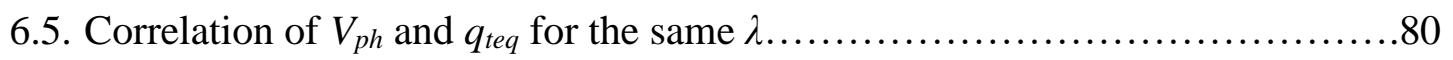

6.6. Limitations...................................................... 81

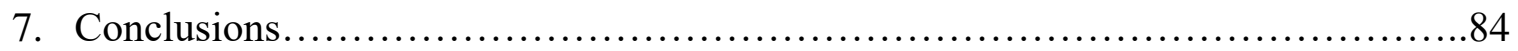

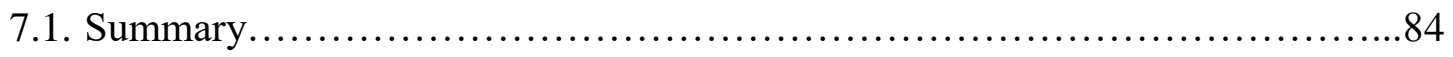

7.2. Recommendations for Future Research................................85

References............................................................. 87

Appendices............................................................... 92

A. Point plots ( $V_{p h}$ vs $q_{t e q}, V_{p h}$ vs $\lambda, q_{t}$ vs $z, q_{t e q}$ vs $\lambda, V_{p h}$ vs $\left.q_{t e q}\right) \ldots \ldots \ldots \ldots \ldots \ldots . \ldots 92$

B. Group plots ( $V_{p h}$ Vs $q_{t e q}, V_{p h}$ vs $\lambda, q_{t}$ Vs $z, q_{t e q}$ Vs $\lambda, V_{p h}$ Vs $\left.q_{t e q}\right) \ldots \ldots \ldots \ldots \ldots \ldots 143$

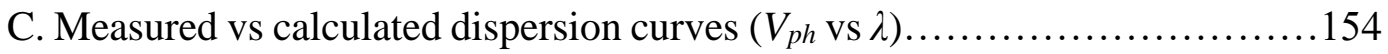

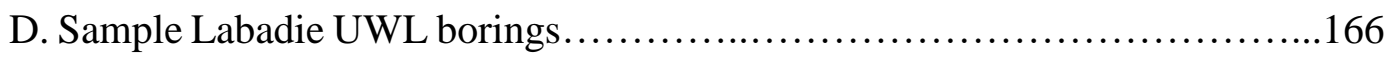




\begin{abstract}
Geotechnical site characterization programs are designed to reduce uncertainty and maximize the efficiency of geotechnical design. Ironically, however, many site characterization programs are inefficient themselves as they call for extensive intrusive testing conducted at regular intervals. This thesis attempts to improve the efficiency of such programs by drawing a correlation between variability in CPT and SASW measurements across a geotechnical site. With such a correlation, the practicing engineer could potentially modify the extent (and cost) of intrusive testing based on observed variability in surface wave dispersion. To examine this correlation, CPT and SASW data from the Labadie Utility Waste Landfill (UWL) near Labadie, Missouri were analyzed. Variability was quantified through coefficients of variation (COV) calculated amongst CPT measurements $\left(q_{t}\right.$ and $\left.f_{s}\right)$ for a particular depth $(z)$ and SASW phase velocity $\left(V_{p h}\right)$ for a particular wavelength $(\lambda)$ at five locations within the UWL. Positive correlations were achieved utilizing a method developed in this thesis wherein $q_{t}$ was converted to an equivalent corrected tip resistance $\left(q_{t e q}\right)$ by weighting $q_{t}$ in the same manner that Rayleigh wave energy is weighted with depth. Utilizing this method, a linear regression between the mean COV of $V_{p h}$ and $q_{t e q}$ yields a slope of 0.18 , an intercept of 0.02 , a coefficient of determination $\left(\mathrm{R}^{2}\right)$ of 0.751 , and a p-value of 0.057 . Removing outliers and COVs calculated in the highly variable upper $5 \mathrm{ft}$ of soil yields a slope of 0.19 , an intercept of 0.02 , a $\mathrm{R}^{2}$ of 0.961 , and a p-value of 0.003 . Finally, a relationship between $q_{t e q}$ and $V_{p h}$ was developed that facilitates the estimation of dispersion curves from $q_{t}$ alone. Utilizing this relationship, dispersion curves estimated from $q_{t}$ were, on average, within $10 \%$ of those measured.
\end{abstract}




\section{Introduction}

\subsection{Background}

At its core, site characterization is about reducing uncertainty in order to enhance geotechnical design. Most commonly, sites are characterized based on existing information (geologic maps, soil surveys, past engineering records, local knowledge, etc.) and a finite number of borings, standard penetration tests (SPT), cone penetration tests (CPT) or other intrusive geotechnical tests. Perhaps counterintuitively, however, the primary objective of many site characterization measures is not only to obtain the engineering properties of the subsurface, but to more generally determine the site's subsurface stratigraphy. Once the general composition of the subsurface is known, a few additional tests to determine engineering properties are often all that is needed for geotechnical design.

Site characterization state-of-practice generally recommends intrusive testing be conducted at regular intervals depending on the site and the type of project (FHWA ED88-053 2003). However, because geologic materials are typically deposited over large expanses relative to geotechnical sites, it is often the case that for a given site, relatively little variation is seen in the soil profile and the engineering properties thereof. Therefore, instead of providing information that leads to substantial changes in geotechnical design, excessive intrusive testing more often serves as a mere validation of subsurface consistency.

This research aims to improve the efficiency of site characterization programs by utilizing a surface wave method (SWM), in this case the Spectral Analysis of Surface Waves (SASW) method, to quantify subsurface variability. Though SWMs only determine the dispersive nature of surface waves, they can provide valuable insight into the spatial 
variability of the subsurface. This is especially true when the inversion process is omitted and phase velocity dispersion curves are considered alone. As stated by Stokoe et al. (1994) “dispersion curves alone can be valuable in quickly evaluating spatial variability at a given site".

\subsection{Objective and Scope of Study}

As intuitive as Stokoe's stated principle is, to date, no specific procedure or research has verified or quantified its utility as a geotechnical site characterization tool. This thesis will evaluate Stokoe's statement by examining variability in SASW dispersion curves relative to variability in CPT measurements. In doing so, this thesis will determine whether SASW dispersion curves, in conjunction with traditional site characterization methods, may improve the efficiency of site characterization practice by reducing the number of intrusive tests needed to sufficiently characterize a site.

Specifically, this thesis sets out to test the following hypothesis: variability in SASW dispersion curves is correlated to variability in CPT measurements. In order to achieve this goal, the following tasks were identified:

- Determine a suitable geotechnical site with extensive CPT data

- Develop an appropriate method to compare variability in SASW dispersion curves and CPT measurements

- Conduct SASW testing

- Quantify variability in SASW and CPT measurements

- Accept or reject the hypothesis

- Provide recommendations for engineering application and further research 


\subsection{Thesis Organization}

Chapter 2 provides a literature review of the state-of-practice for SASW and CPT testing as well as their current role in site characterization. A brief description of the Labadie UWL site including the geologic history, generalized soil profiles, and geotechnical testing history is provided in Chapter 3. Chapter 4 outlines the methods utilized during CPT and SASW testing as well as those applied to data reduction and variability analyses. Results of CPT, SASW, and variability analyses are presented in Chapter 5. Chapter 6 includes discussion of the methodology, results, and limitations of the study. Finally, a summary of this research project and the conclusions drawn from it are presented in Chapter 7. 


\section{Literature Review}

\subsection{Overview}

Literature regarding a direct correlation between variability in SASW dispersion curves and CPT measurements is not currently available. However, much work has been done that considers SASW and CPT independently with respect to site characterization. Section 2.2 provides an overview of SASW as well as its limitations, advancements, and current use in site characterization. Section 2.3 is a brief overview of the current state of practice of CPT. Finally, Section 2.4 summarizes state-of-practice site exploration guidelines for intrusive testing.

\subsection{Spectral Analysis of Surface Waves (SASW)}

Spectral Analysis of Surface Waves (SASW) was pioneered in the early 1980s following the advent of field-portable digital data acquisition and signal-processing equipment. The principal utility of SASW is that shear wave velocities are directly proportional to the stiffness of the material through which they propagate. Thus, from them intrinsic properties of engineering significance may be obtained. In the 1980's and 90's the most common method for analyzing shear waves in geo-materials was cross-hole and down-hole methods. However, both of these techniques are intrusive, costly, and time consuming. Because SASW measures the propagation of Rayleigh waves at the surface, it is significantly more cost effective (Stokoe et al. 1994).

Before SASW, the steady-state Rayleigh wave method was the primary surface method used to obtain a shear wave velocity profile. For this method, vibrations are emitted at constant frequency and sensors are moved further and further away from each other until 
they are in phase. When two receivers are in phase, shear wave velocities are obtained by simply multiplying the frequency by the receiver spacing. After repeating this process for several frequencies, velocities are plotted against their respective frequency or wavelength and a dispersion curve takes form (Stokoe et a. 1994).

As is outlined in Stokoe et al. (1994), by 1994 advances in data analysis and on-site computing made it possible to use impulse, swept-sinusoidal, or random noise as inputs. Utilizing two receivers and a data analyzer, a Fast Fourier Transform (FFT) is performed on-site and the input signal from each receiver is separated into individual frequencies with varying phases. The phase difference $(\varphi)$ for each frequency $(f)$ between the two receivers is then calculated and travel time $(t)$ between receivers for each frequency can be determined (Eq. 2.1). It then follows that the Rayleigh wave velocity $\left(V_{R}\right)$ for a given frequency is the receiver spacing $(S)$ (assuming the distance from the first receiver to the source is the same) divided by the travel time (Eq. 2.2). Finally, the wavelength $\left(\lambda_{R}\right)$ for each frequency is obtained by dividing $V_{R}$ by the frequency (Eq. 2.3). This process is carried out for the available range of frequencies and a dispersion curve is produced.

$$
\begin{aligned}
& t(f)=\frac{\varphi(f)}{2 \pi f} \\
& V_{R}=\frac{S}{t(f)} \\
& \lambda_{R}=\frac{V_{R}}{f}
\end{aligned}
$$

In SASW, shear wave velocity $\left(V_{s}\right)$ profiles are achieved by forward modeling potential velocity profiles to fit the measured dispersion curve. The $V_{s}$ profile is therefore not a unique solution, but one of several possible solutions. Further, because Rayleigh waves must propagate through shallow layers on their way to deeper layers, resolution of 
shear wave velocities decreases with depth. As a rule of thumb, SASW is only able to discern soil/rock layers with thicknesses greater than about $20 \%$ of the depth (Stokoe et al. 1994). That is, at $10 \mathrm{ft}$ below the surface, one cannot expect to discern layers thinner than $2 \mathrm{ft}$. Finally, it is important to note that SASW is not a point measurement. Rather it provides the globally averaged $V_{s}$ for given depths as measured from the source to the furthest receiver.

\subsubsection{SASW \& Site Characterization}

The SASW method outlined by Stokoe et al. (1994) was one of the most widely used surface methods in geotechnical site characterization. However, during the late 90's researchers made a number of improvements to surface wave methods (SWM) which eventually led to the development of Multichannel Analysis of Surface Waves (MASW). These improvements included advances in spatial array processing, using low-frequency passive energy, utilizing multiple modes of wave propagation, and data smoothing (Rix et al. 2001). Though only one of these methods (smoothing) was implemented while using SASW for this thesis, all have enhanced the potential of surface waves for site characterization.

Today, the most common application of SWM in site characterization is seismic site response analysis. However, until recently doubt has existed in the geotechnical community as to the suitability of surface methods for such analyses. The chief complaint is that lack of resolution and non-uniqueness in $V_{s}$ profiles can yield unconservative site response spectra. Foti et al. (2009) disproved this notion showing that while subsurface

profiles, as determined by SWM, are non-unique and sensitive to initial model inputs, they 
still provide an accurate global account of seismic site response. The underlying principle is that sites with similar global surface wave propagation are likely to be similar with respect to seismic response. That is to say, sites with similar dispersion curves will behave similarly during seismic events.

In order to test their hypothesis, the authors utilized robust Monte Carlo analyses of a synthetic site as well as two actual sites. The analyses were performed by creating several random models that fit a given dispersion curve. Figure 2.1 shows six shear wave velocity profiles that were randomly generated to fit a given dispersion curve. Each of these profiles were then analyzed in Shake91 for seismic site response. Figure 2.2 shows that the difference in seismic site response for all six profiles is negligible. From all of the analyses performed, it was determined that response spectra and amplification factors for all randomly generated sites fall within 5-10\% of the exact solution. Similar Monte Carlo simulations were created for two actual sites, Torre Pellice and La Salle, Italy which both yielded the same results as the synthetic case.

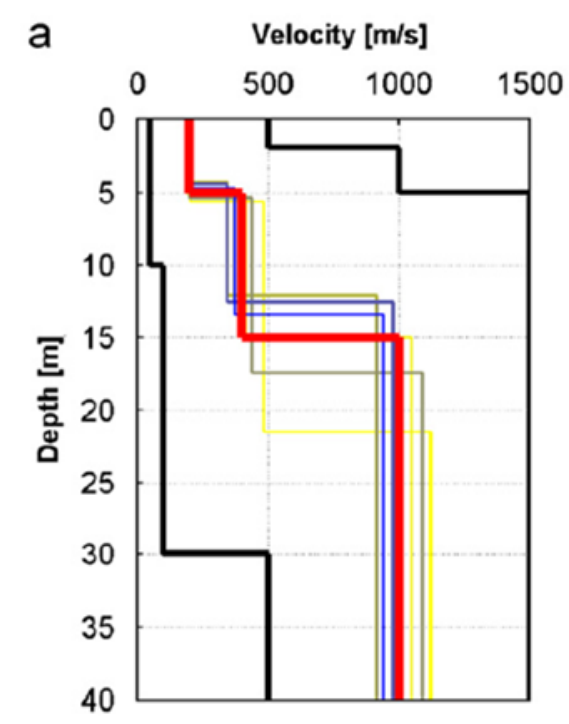

b

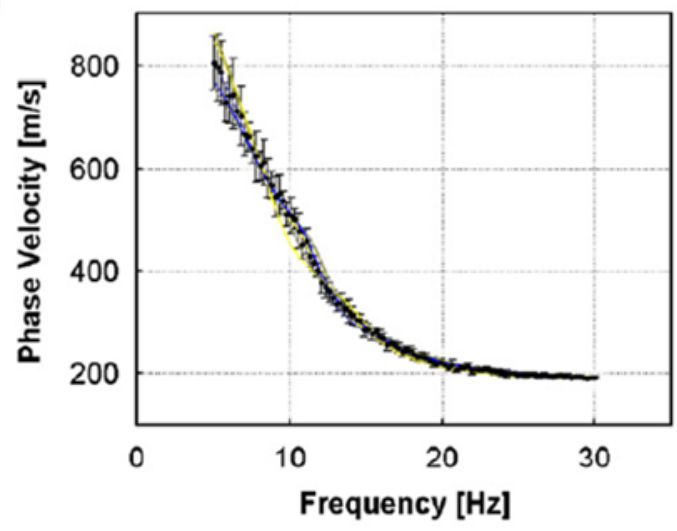

Figure 2.1 - a.) Six randomly generated velocity profiles b.) Corresponding dispersion curves (Foti et al. 2009). 

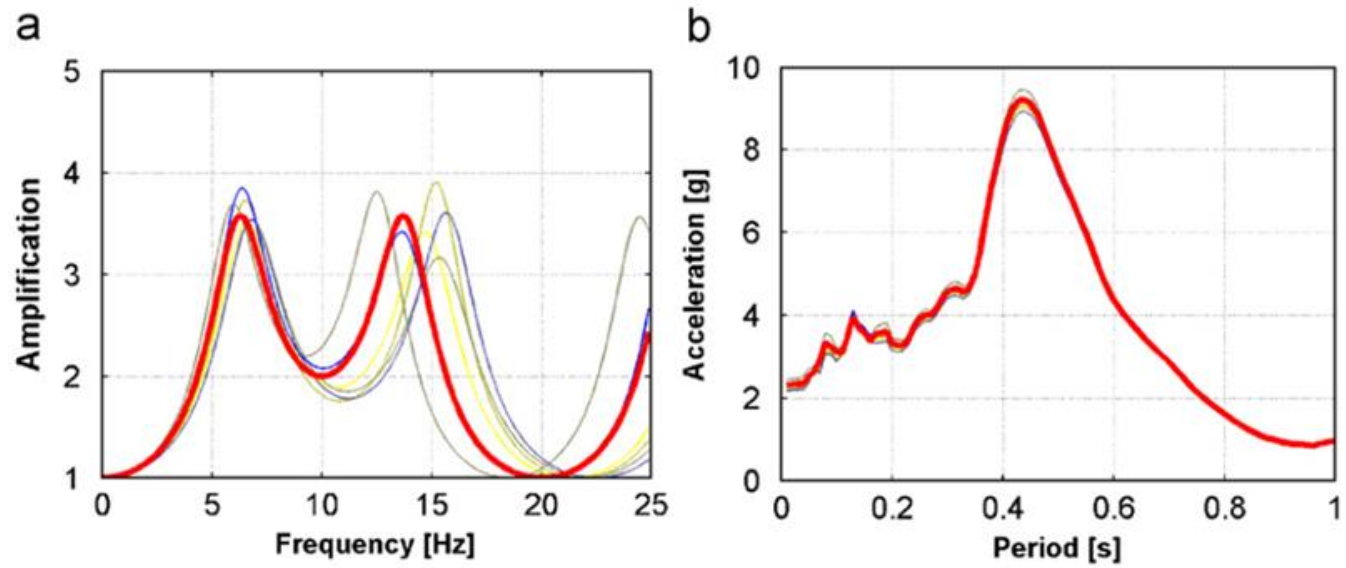

Figure 2.2 - a.) Amplification functions b.) Response spectra (Foti et al. 2009).

In conducting their study, Foti et al. (2009) countered the most widespread criticism of SWM for seismic site response. While it is certain that stiffness and velocity profiles achieved via SWM are not unique solutions, their non-uniqueness bears little significance with respect to seismic performance. That is to say, if sites are equivalent with respect to Rayleigh wave dispersion, they too are equivalent with respect to seismic site response. Furthermore, SWM are well-suited for such investigations.

\subsubsection{SASW Summary}

For nearly 60 years, surface waves have been used to characterize geotechnical sites. Advances in data analysis and computing eventually facilitated on-site FFT analyses and SASW supplanted the steady-state Rayleigh wave method. State-of-the-art SWM now incorporates MASW utilizing both active and passive energy. With respect to site characterization, SWM are typically used to supplement data obtained from conventional (SPT, CPT, borings, etc.) analyses or serve as a baseline for choosing the type and quantity of conventional site investigation methods. They are particularly effective in terms of 
seismic site response and recent research has discredited claims that solution nonuniqueness may yield unconservative site response spectra.

\subsection{Cone Penetration Test}

The cone penetration test (CPT) is a widely-used, efficient, and reliable method for geotechnical site characterization. Today, most CPT systems are outfitted with pore-water pressure transducers and are referred to as a piezocone penetration tests (CPTU) (Figure 2.3). Another common CPT variation is the seismic piezocone penetration test (SCPTU) which includes at least one geophone and is capable of measuring shear wave velocities with depth. Less common are CPT systems capable of measuring resistivity (RCPTU) or taking photographs or video of the soil at depth.

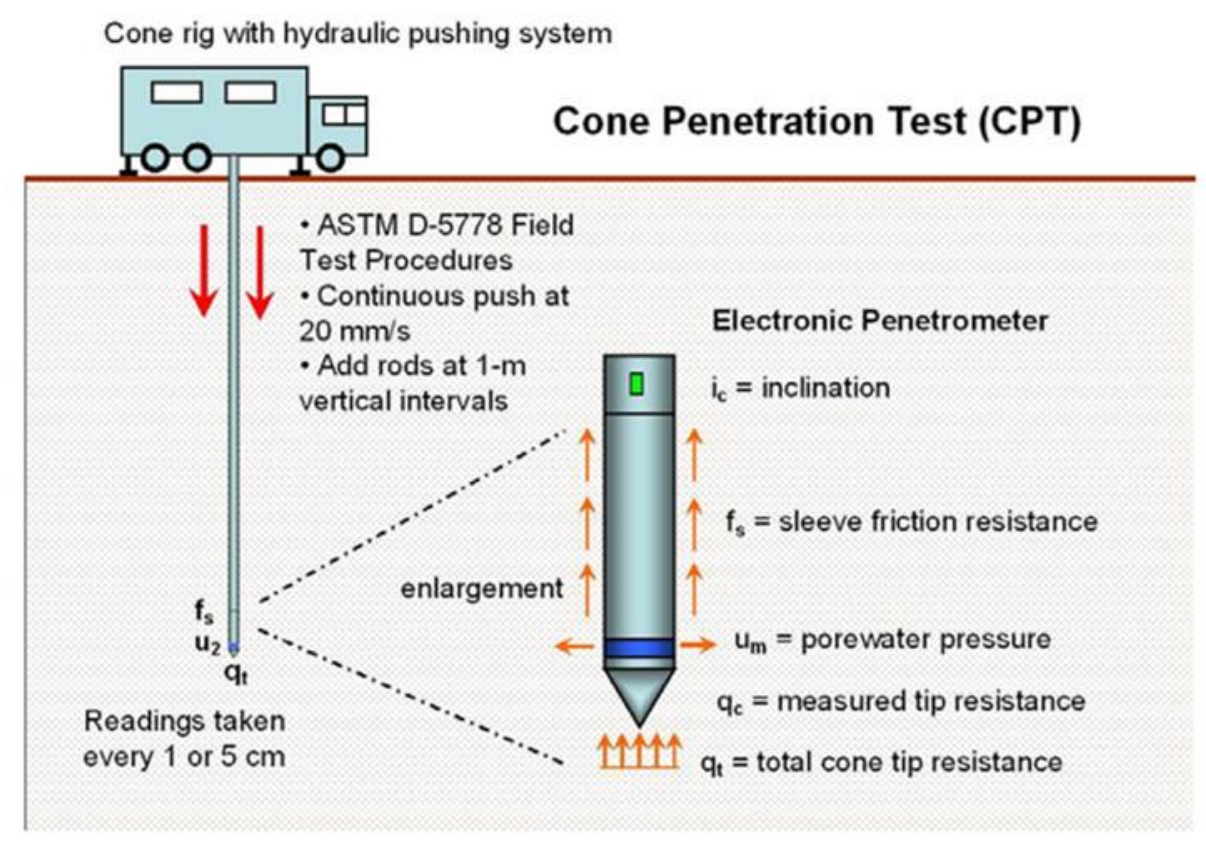

Figure 2.3 - Cone penetration test (Mayne 2007). 
From the three primary CPTU measurements $\left(q_{c}, f_{s}, u_{m}\right)$ a wide range of correlations for geotechnical parameters (soil type, unit weight, stress history, shear wave velocity, undrained shear strength, friction angle etc.) have been well-established. Economically the CPT is quite efficient with costs ranging from $\$ 6$ to $\$ 13.5$ per foot depending on test size, location, material specific requirements, and post-grouting etc. (Mayne 2007). This compares to $\$ 12$ to $\$ 33$ per foot for traditional borings, depending on similar factors, making the CPT about half the cost of a traditional boring (Mayne 2007).

Several correlations have also been developed for estimating $V_{s}$ from CPT measurements. While many are available for all soils, most have been developed for a specific soil type and are functions of various combinations of $q_{c}, q_{t}, f_{s}$, confining stress $\left(\sigma_{v}\right)$, effective confining stress $\left(\sigma_{v}^{\prime}\right)$, and depth (Table 2.1). Overall, the range of equations and their coefficients of determination vary widely and are ultimately a function of the applied methods and input data. The theoretical relationship between $V_{s}$ and CPT measurements resides in the fact that many of the factors that affect small-strain $\left(G_{m a x}\right)$ and large-strain stiffness are the same. Hardin and Drnevich (1972) describe $G_{\max }$ as a function of void ratio (e), mean principal effective stress $\left(\sigma_{o}^{\prime}\right)$, and overconsolidation ratio (OCR), all of which affect large-strain stiffness in the same manner. That is, both small-strain and large-strain moduli increase with decreasing $e$ and increasing $\sigma_{o}^{\prime}$ and OCR. Other soil properties that have proportional effects on large and small-strain moduli include soil type, geologic age, and cementation (Wair, DeJong, \& Shantz 2012). 
Table 2.1 - CPT- $V_{s}$ correlations (from Wair et al. 2012).

\begin{tabular}{|c|c|c|c|c|c|c|}
\hline Soil Type & Study & Geologic Age & $\begin{array}{l}\text { Number of } \\
\text { Data Pairs } \\
\end{array}$ & $\mathrm{r}^{2}$ & $V_{\mathrm{S}}(\mathrm{m} / \mathrm{s})$ & $(\mathrm{Eq} \#)$ \\
\hline \multirow{5}{*}{ All Soils } & Hegazy \& Mayne (1995) & Quaternary & 323 & 0.70 & $\left(10.1 \log \left(\mathrm{q}_{2}\right)-11.4\right)^{1.67}\left(100 \mathrm{f}_{3} / \mathrm{q}_{2}\right)^{0.3}$ & $(5.6)$ \\
\hline & Mayne (2006) & Quaternary & 161 & 0.82 & $118.8 \log \left(\mathrm{f}_{\mathrm{s}}\right)+18.5$ & $(5.7)$ \\
\hline & Piratheepan (2002) & Holocene & 60 & 0.73 & $32.3 \mathrm{q}^{0.099} \mathrm{f}_{\mathrm{s}}^{0.121} \mathrm{D}^{0.215}$ & $(5.8)$ \\
\hline & Andrus et al. (2007) & $\begin{array}{c}\text { Holocene \& } \\
\text { Pleistocene }\end{array}$ & 185 & \begin{tabular}{|l|} 
(H) 0.71 \\
(P) 0.43 \\
\end{tabular} & $2.62 \mathrm{q}_{\mathrm{t}}^{0.395} \mathrm{I}_{\mathrm{C}}^{0.912} \mathrm{D}^{0.124} \mathrm{SF}^{d}$ & $(5.9)$ \\
\hline & Robertson (2009) & Quaternary & 1,035 & - & {$\left[\left(10^{(0.551 \mathrm{r}+1.68)}\right)\left(\mathrm{q}_{\mathrm{t}}-\sigma_{\mathrm{v}}\right) / \mathrm{p}_{\mathrm{z}}\right]^{0.5}$} & $(5.10)$ \\
\hline \multirow{5}{*}{ Sand } & Sykora \& Stokoe (1983) & - & 256 & 0.61 & $134.1+0.0052 \mathrm{q}_{\mathrm{c}}$ & $(5.11)$ \\
\hline & Baldi et al. (1989) & Holocene & - & - & $17.48 \mathrm{q}_{\mathrm{e}}^{0.13 \sigma_{\mathrm{v}} 0.27}$ & $(5.12)$ \\
\hline & Hegazy \& Mayne (1995) & Quaternary & 133 & 0.68 & $13.18 \mathrm{q}^{0.192} \sigma_{\mathrm{v}}^{0.179}$ & $(5.13)$ \\
\hline & Hegazy \& Mayne (1995) & Quaternary & 92 & 0.57 & $12.02 \mathrm{q}_{\mathrm{e}}^{0.319} \mathrm{f}_{\mathrm{s}}^{-0.0466}$ & $(5.14)$ \\
\hline & Piratheepan (2002) & Holocene & 25 & 0.74 & $25.3 \mathrm{q}^{0.163} \mathrm{f}_{\mathrm{s}}^{0.029} \mathrm{D}^{0.155}$ & $(5.15)$ \\
\hline \multirow{5}{*}{ Clay } & Hegazy \& Mayne (1995) & Quaternary & 406 & 0.89 & $14.13 \mathrm{q}_{\mathrm{c}}^{0.359} \mathrm{e}_{0}^{-0.473}$ & $(5.16)$ \\
\hline & Hegazy \& Mayne (1995) & Quaternary & 229 & 0.78 & $3.18 \mathrm{q}_{\mathrm{c}}^{0.549} \mathrm{f}_{\mathrm{s}}^{0.025}$ & $(5.17)$ \\
\hline & Mayne \& Rix (1995) & Quaternary & 339 & 0.83 & $9.44 \mathrm{q}^{0.435} \mathrm{e}_{0}^{-0.532}$ & $(5.18)$ \\
\hline & Mayne \& Rix (1995) & Quaternary & 481 & 0.74 & $1.75 \mathrm{q}^{0.627}$ & $(5.19)$ \\
\hline & Piratheepan (2002) & Holocene & 20 & 0.91 & $11.9 \mathrm{q}_{\mathrm{c}}^{0.269} \mathrm{f}_{\mathrm{s}}^{0.108} \mathrm{D}^{0.127}$ & $(5.20)$ \\
\hline
\end{tabular}

Units: $q_{c}, q_{v}, f_{v}, \sigma_{v}$, and $\sigma_{v}$ are measured in kilopascals $(\mathrm{kPa})$, and depth $(\mathrm{D})$ is measured in meters $(\mathrm{m}) . \mathrm{p}_{\mathrm{a}}=100 \mathrm{kPa}$.

${ }^{\mathrm{s}} \mathrm{SF}=0.92$ for Holocene and 1.12 for Pleistocene

\subsection{Boring and Sampling in Site Characterization Practice}

Of particular interest to this study is the manner in which inherent uncertainties are dealt with in practice. In cases where deterministic design is applied, inherent uncertainties are generally handled by incorporating factors of safety and conducting boring and sampling on a regular interval. Less commonly, reliability-based design methods simultaneously account for inherent and measurement uncertainties based on statistical 
models of soil parameters and, even less commonly, soil stratigraphy. These methods also require boring and sampling on a regular, and often smaller interval.

The Federal Highway Administration (FHWA) outlines minimum standards for boring and sampling in FHWA ED-88-053 (2003) (Table 2.2). The FHWA publication also specifies that rigid rules cannot be established and the guidelines contained therein are considered the reasonable minimum; ultimately leaving the problem to engineering judgement. Table 2.2 shows that borings are generally recommended every hundred to few hundred feet depending on the type of project. The FHWA publication goes on to recommend types of borings depending on the specific geomaterial. For sands, SPTs are recommended with samples taken every $5 \mathrm{ft}$ or at major changes in soil strata. For clays, SPT and thin wall tube samples should also be taken every $5 \mathrm{ft}$ and field vane tests are recommended to obtain in-situ shear strengths. For rock, continuous cores should be taken. Finally, for borrow sites any equipment that allows direct observation of the material is recommended. 


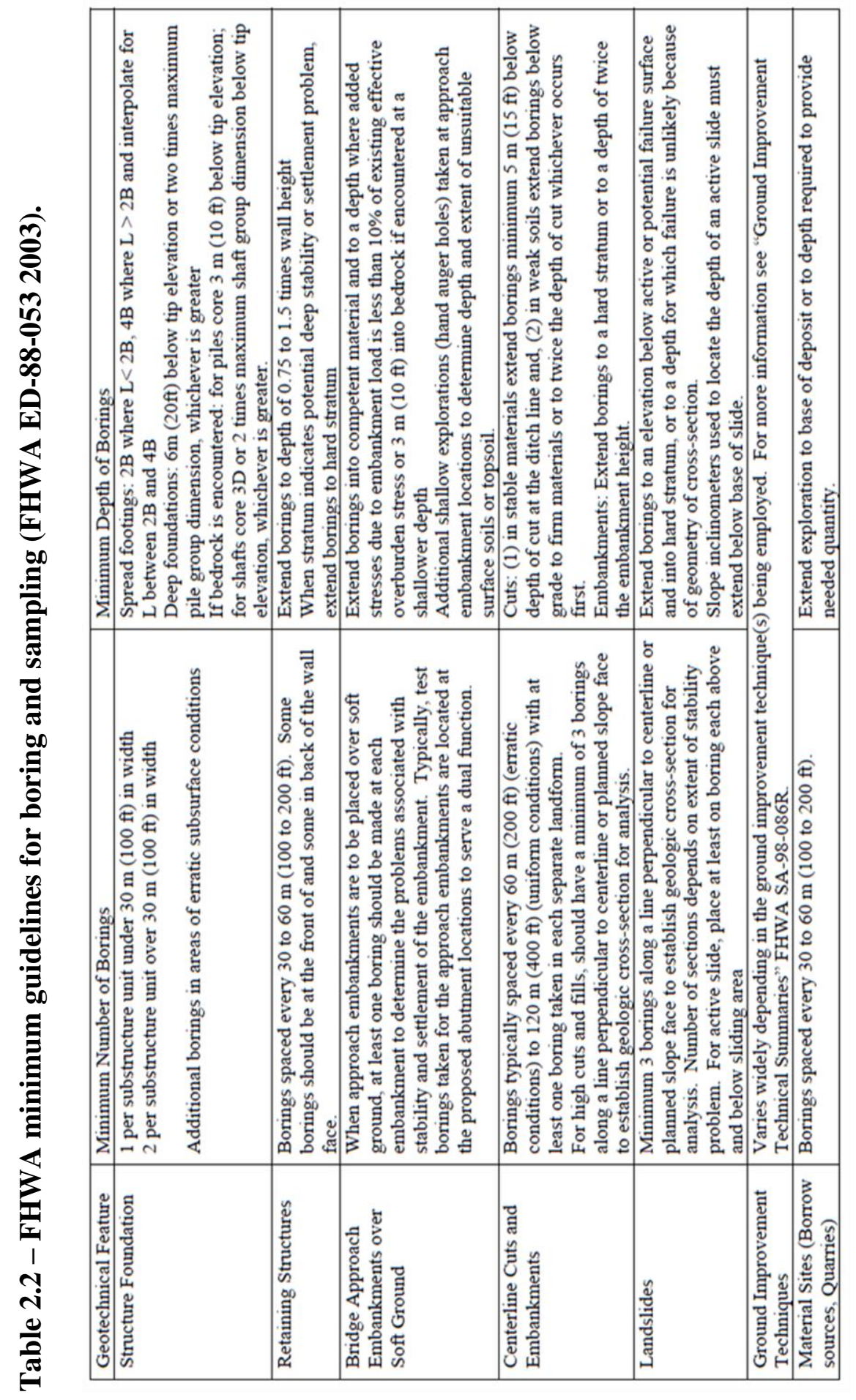




\subsection{Summary}

In order to best compare variability in SASW dispersion curves and CPT measurements, it is important to examine the state-of-practice of each. Since the 1980's, SWM have been a cheap and reliable method for non-intrusive subsurface investigation. Recent developments in SWM such as spatial array processing, data smoothing, and utilization of low-frequency passive energy and multiple modes of wave propagation have significantly enhanced SWM capabilities (Rix et al. 2001). Furthermore, work by Foti et al. (2009) shows that inverted shear wave velocity profiles are less significant to seismic site response than the dispersive nature of the soil.

Similar to SWM, CPT technology continues to improve and now incorporates continuous and precise sampling of upwards of ten independent measurements (Mayne 2007). Combined with advancements in data processing, CPT now provides what is arguably the most cost-effective and reliable site exploration tool in the geotechnical inventory. The efficiency with which CPT (and SWM) are used for site characterization, however, has seen little change. Advanced methods for geotechnical site characterization such as the Bayesian approach and random field theory have been developed, but are seldom used in practice. Ultimately, state-of-practice site characterization continues to handle inherent uncertainty through broad recommendations and engineering judgement. 


\section{Site Description}

\subsection{Introduction}

Several factors were considered while identifying potential geotechnical sites for this study. First, in order to perform enough testing to make a statistically significant correlation, a large site (at least 100 acres) with several CPT soundings taken at regular intervals was preferred. The site should also have relatively flat topography in order to make comparison of spatial variability more simplistic. Consistent groundwater conditions were also desirable so that the fairest comparison may be made between recent SASW measurements and older CPT soundings. Finally, the site should be relatively free from excessive vegetation, standing water, be accessible for foot and vehicular traffic, and have otherwise favorable surface conditions for SASW testing.

\subsection{Labadie Utility Waste Landfill (UWL) Site}

Fortunately for this study, the Labadie Power Plant utility waste landfill (UWL) meets virtually all of the desirable site criteria. The plant is located on the Missouri River floodplain approximately 2.5 miles northeast of Labadie, Missouri. It is owned and operated by Ameren Missouri, an electric and gas utility company, headquartered in St. Louis, Missouri. In May of 2009, a detailed site investigation (DSI) plan was approved by the Missouri Department of Natural Resources (MDNR) for a UWL adjacent to the plant for the disposal of coal combustion residuals (CCR). The plan was prepared by Reitz \& Jens, Inc. of St. Louis, Missouri and GREDELL Engineering Resources, Inc. of Jefferson City, Missouri. The field investigation began in September 2009 and was completed in January 2010 and the DSI report was submitted in February 2011. Today, 
the site remains largely unused with only the westernmost 40 acres of the site being used for the UWL. The remainder of the site is currently being leased for agricultural purposes.

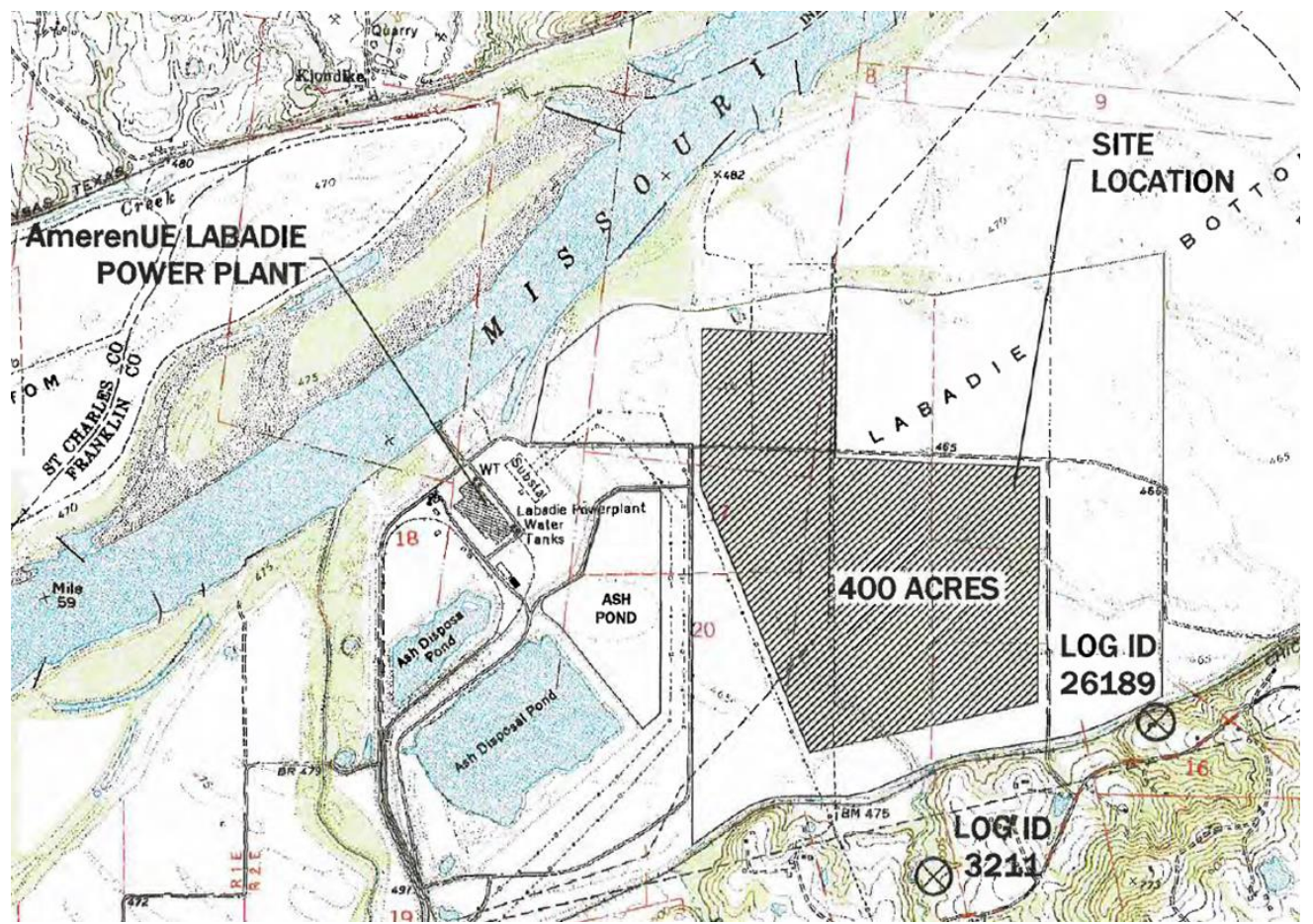

Figure 3.1 - Labadie UWL site (GREDELL Engineering Resources, Inc. and Reitz \& Jens, Inc. 2011).

As described in the 2011 DSI report by GREDELL Engineering Resources, Inc. and Reitz \& Jens, Inc., the UWL site is approximately 400 acres of Holocene Missouri River alluvial deposits. The site is remarkably flat with total topographic relief less than $10 \mathrm{ft}$. The site is bounded to the north by a flood levee and Labadie Bottom Road, to the east by Davis Road, to the west by Labadie Bottom Road, and to the south by Becker Creek (Figure 3.1). As is typical of Missouri River alluvial deposits, the upper $15 \mathrm{ft}$ generally consist of fine sands, silts and clays. From 15 to $50 \mathrm{ft}$, fairly homogenous course grained 
sands are prevalent. From 50 to $100 \mathrm{ft}$, sandy gravels, gravel, and even cobbles are present as particle size generally increases with depth. Middle Ordovician sedimentary deposits consisting of dolomites with occasional beds of limestone and sandstone are encountered at approximately $100 \mathrm{ft}$ across the site. Finally, 2007 boring records have the groundwater table at 10 to $20 \mathrm{ft}$ below the ground surface. More recent piezometer readings from June 2016 have the groundwater table ranging from about 5 to $9 \mathrm{ft}$, and those from December 2016 have it at 9 to $18 \mathrm{ft}$.

The field investigation was initiated in the summer of 2009 and completed in January of 2010 under the supervision of senior geologist Mikel Carlson, R. G., of GREDELL Engineering Resources, Inc. and senior project manager Jeffrey Fouse, P. E., of Reitz \& Jens, Inc. In total, it consisted of 24 borings, 76 CPT soundings, and the instillation of 100 piezometers, all of which were done in accordance with applicable ASTM standards and spaced on a grid pattern across the 400-acre site (Figure 3.2). All borings and CPT soundings were taken to a minimum depth of $35 \mathrm{ft}$ which is approximately $20 \mathrm{ft}$ below the proposed depth of the UWL. Three borings were extended to auger refusal which occurred at approximately $100 \mathrm{ft}$ (Appendix D). For cases where cone refusal occurred at less than $35 \mathrm{ft}$, a second sounding was taken in an attempt to increase penetration.

Finally, a few randomly selected CPT soundings were verified by performing an additional sounding or boring at approximately the same location. All samples were logged in accordance with applicable ASTMs and moisture content tests were performed on all fine-grained samples. Additional testing such as unconsolidated-undrained and consolidated-undrained triaxial tests, one-dimensional consolidation tests, and flexiblewall hydraulic conductivity tests were conducted at the discretion of the senior project 
manager in accordance with stated site characterization goals (GREDELL Engineering Resources, Inc. and Reitz \& Jens, Inc. 2011). 


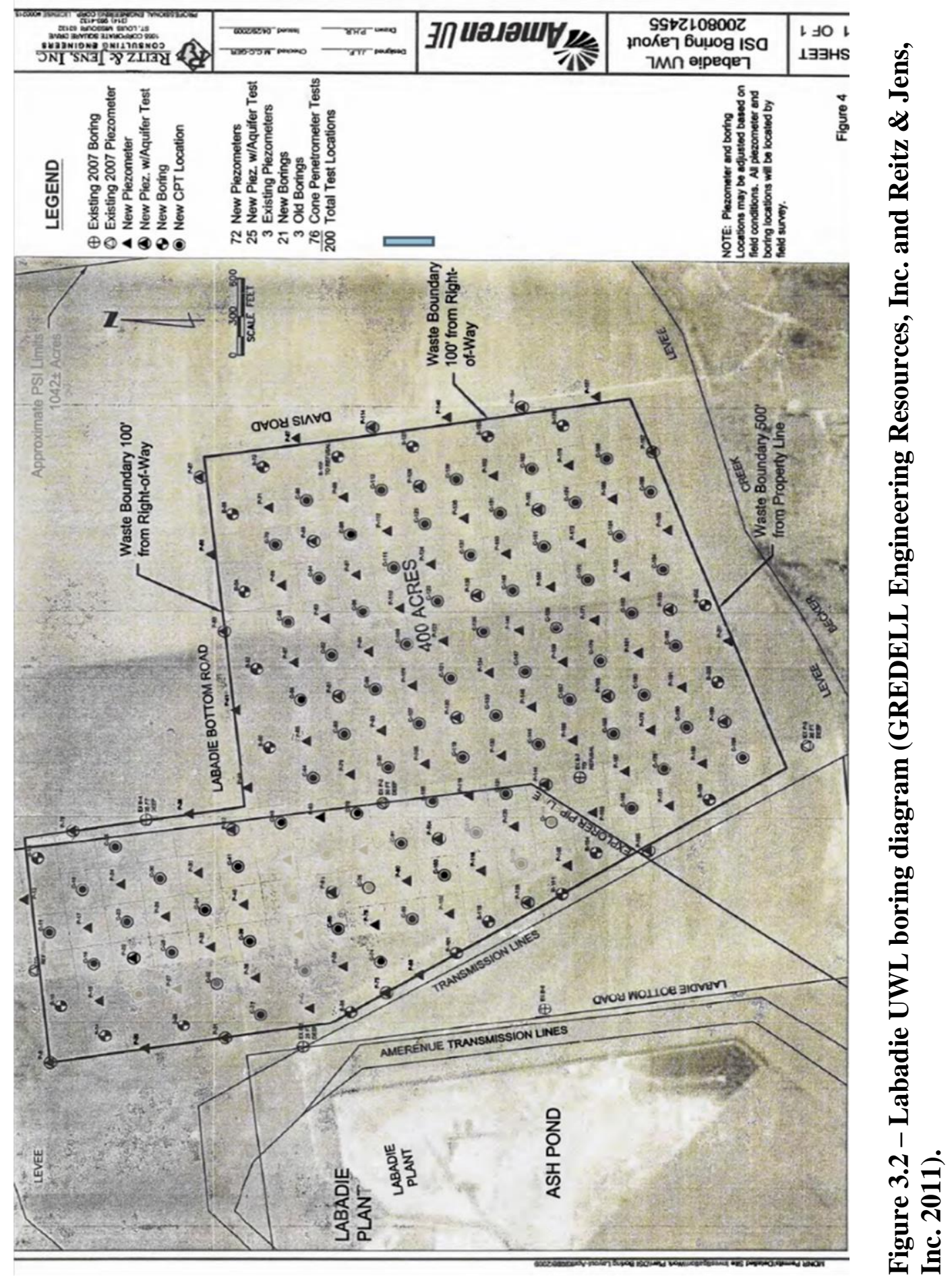




\subsection{Subdivision of the UWL}

Correlation of variability in SASW dispersion curves and CPT tip resistance is best examining utilizing several geotechnical sites. However, field testing at several geotechnical sites was impractical due to this study's limited budget. The UWL site was therefore broken into several smaller "sites" which will be referred to as "groups" from this point forward. In total, six, eight-acre groups consisting of at least five points where CPT soundings were performed were created (Figure 3.3). Groups were chosen based on size, availability of CPT soundings, and the need to have enough groups to determine a statistically significant variability correlation. All points within the groups are numbered consistent with the 2011 DSI report and groups are numbered based on their center point. Because there are points where more than one CPT sounding was taken, some groups contain more than 5 points. For example, Group 80 consists of Points 64, 66, 66A, 80, 92, and 94, however tests 66 and 66A were done at approximately the same location.

The northernmost group, and the only group north of Labadie Bottom Road, is Group 23 and consists of Points 16, 18, 23, 28, and 30. Group 80 is the northwesternmost group south of Labadie Bottom Road, and as previously mentioned consists of Points 64, 66, 66A, 80, 92, and 94. Group 84 is the northeasternmost group and consists of Points 68, 70, 84, 96, and 98. Group 135 is the center group south of Labadie Bottom Road and includes Points 121, 123, 135, 135A, 147, 147A, and 149. The southwesternmost group is Group 180 and consists of Points 168, 168A, 170, 180, 190, and 192. Finally, the southeasternmost group is Group 184 and includes Points 172, 174, 184, 194, and 196. A more detailed diagram with exact locations for each point is also provided in Chapter 4. 


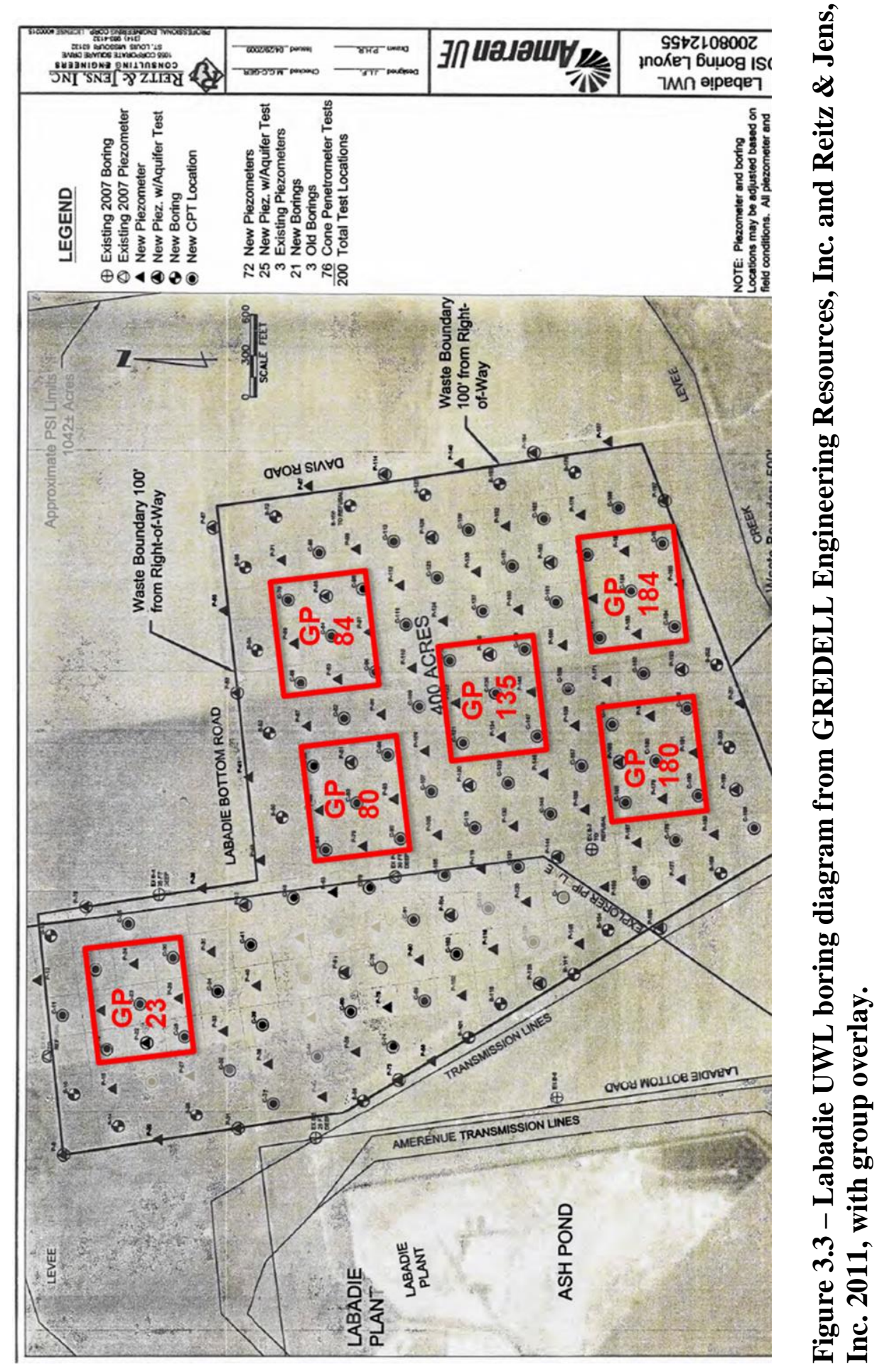




\section{Methods}

\subsection{Overview}

This chapter provides a detailed account of the methods utilized to determine a correlation between variability in SASW dispersion curves and CPT measurements. CPT procedures used by Reitz \& Jens, Inc. are briefly outlined in Section 4.2. SASW field testing procedures, data reduction and dispersion curve fitting are provided in Sections 4.3 and 4.4. The preliminary method utilized for quantifying variability in SASW dispersion curves and CPT data is explained in Section 4.5. Finally, an explanation of the equivalent wavelength method (EWM) and an example variability quantification are provided in Section 4.6.

\subsection{Cone Penetration Testing}

As is detailed in the 2011 DSI report, cone penetration testing was conducted in October, November, and December of 2009 by Reitz \& Jens, Inc. All tests were conducted in accordance with ASTM D5778 "Electronic Friction Cone and Piezocone Penetration Testing of Soils" utilizing an AMS-probe rig with 1.5-in cone. The CPT rig was operated by Terra Drill, Inc. of Dupo, Illinois under the supervision of Reitz \& Jens geotechnical engineer Christopher Cook, P. E. For this thesis, the processed CPT measurements provided in the 2011 DSI report were utilized. Prior to their utilization, processed measurements were selectively verified from raw data and found to be correct in all cases. 


\subsection{SASW Testing}

A total of 29 SASW tests were conducted at the Labadie UWL site on the 19th, 21st, and 22nd of November 2016. Figure 4.1 shows the location, general surface conditions, and outcome of each planned test. Testing began in the northwestern corner of the site and progressively moved south and east. Weather was fairly consistent each day with highs around $45^{\circ}$ and lows around $30^{\circ}$ with no precipitation. On the 19th, wind was approximately $10 \mathrm{mph}$ out of the northwest, while winds on the $21 \mathrm{st}$ and 22 nd were calm.

Originally, 34 tests were planned but heavy overgrowth in the northeastern section made SASW testing impractical (Figure 4.1). Of the 29 tests, 26 resulted in usable dispersion curves. Dispersion curves for Points 123 and 147 could not be identified due to scatter in phase diagrams, and incorrect test procedures resulted in inverted data for Point 92.

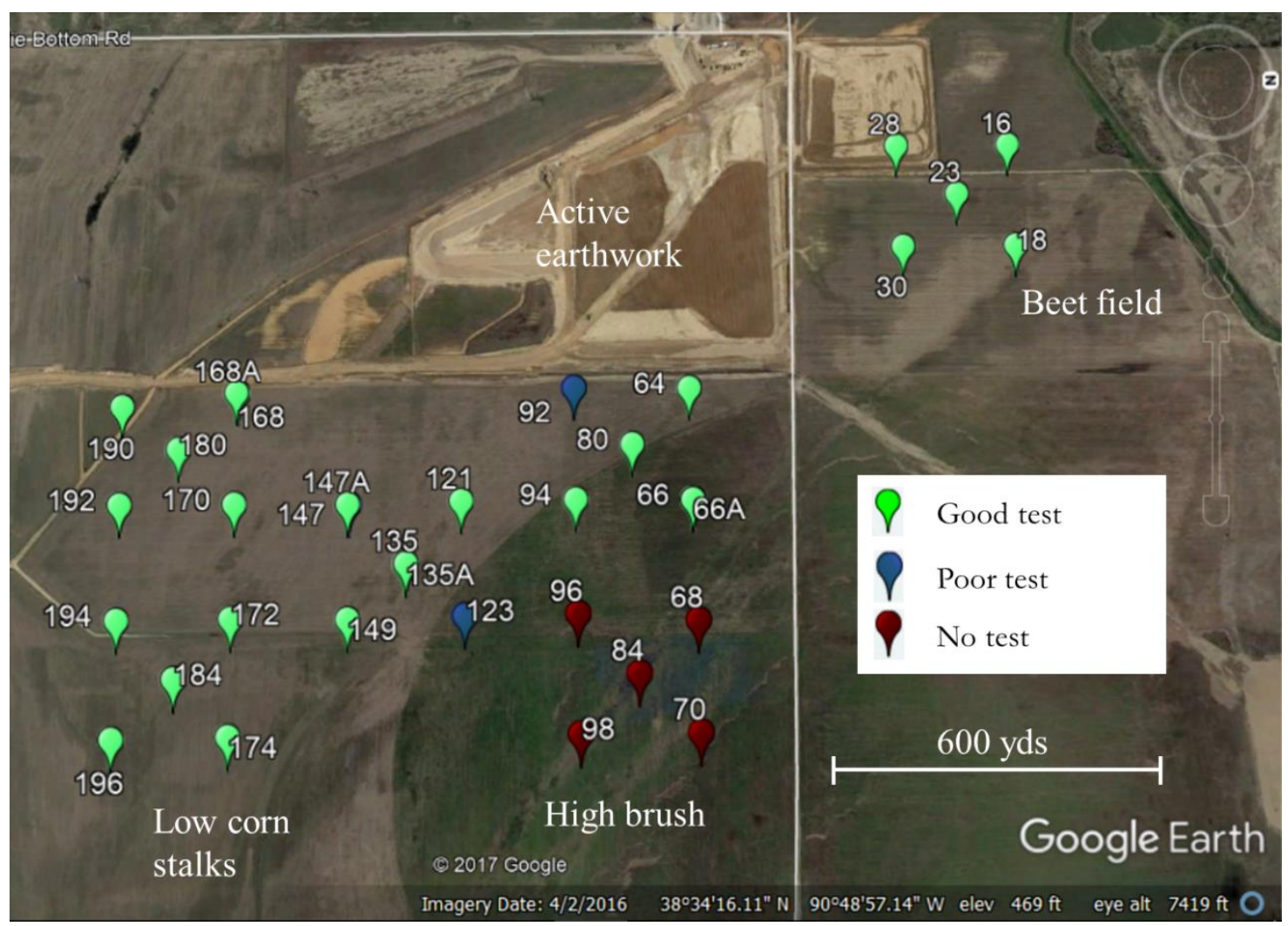

Figure 4.1 - Labadie UWL SASW testing overview. 


\subsubsection{Equipment}

SASW testing was completed utilizing a SignalCalc ACEII dynamic signal analyzer version 4.8.309 and Geospace GS-11D 4.5 Hz rotating coil geophones. Geophones were housed in waterproof hard cases with 3-in spikes and connected with $300 \mathrm{ft}$ of twoconductor, twisted, shielded pair wire (Figure 4.2). A 100-ft tape was used to mark source and receiver locations and a 10-lb sledge hammer and 50-lb drill bit were used to generate surface waves. Prior to field testing, all geophones were checked for phase consistency.

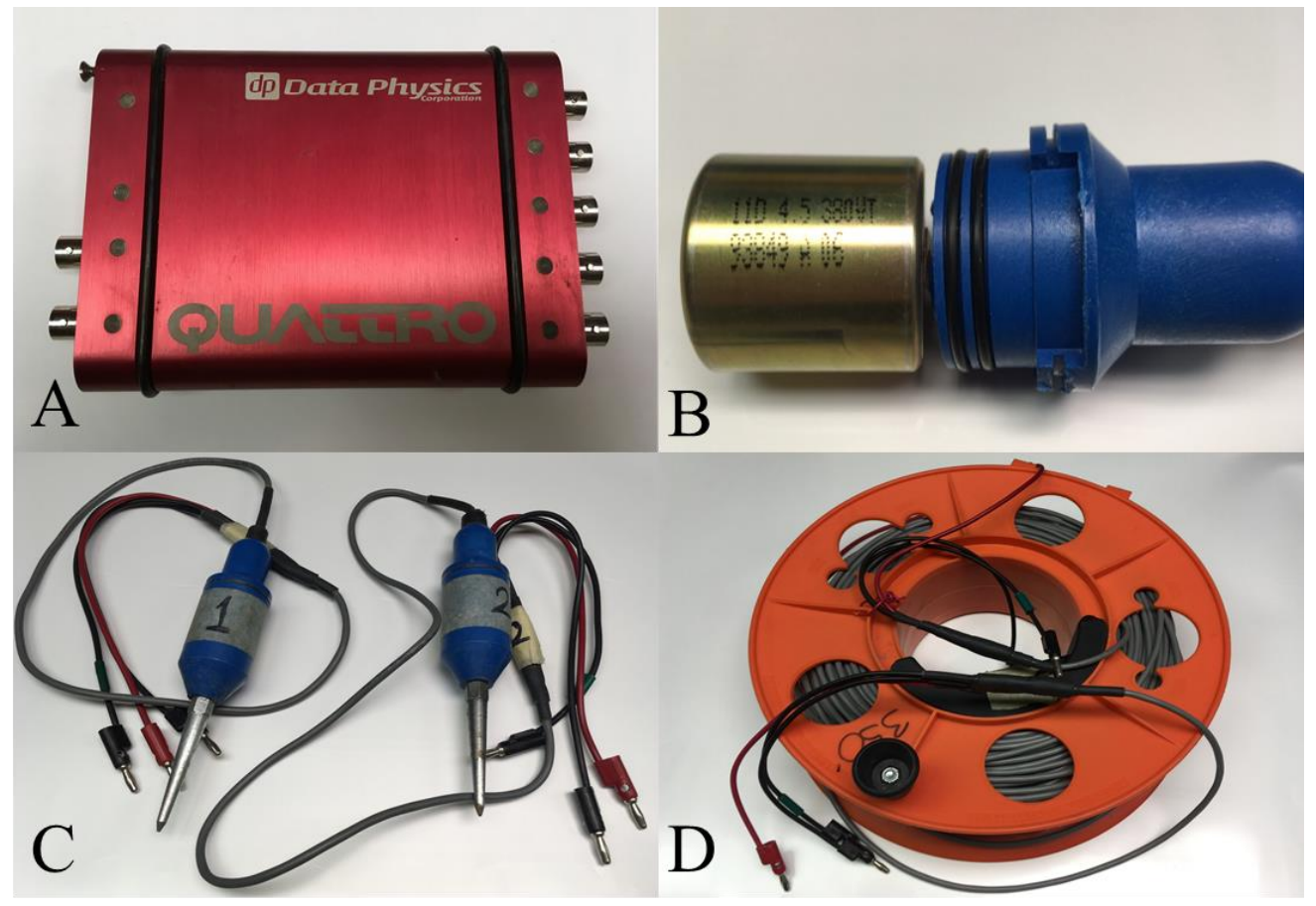

Figure 4.2 - SASW testing equipment A.) Data Physics signal analyzer B.) Geospace $4.5 \mathrm{~Hz}$ geophone $\mathrm{C}$.) Geophones in waterproof casing with seating spikes D.) $300 \mathrm{ft}$ of two-conductor, twisted, shielded pair wire. 


\subsubsection{Procedures}

SASW tests were carried out at 29 points following the procedure outlined by Stokoe et al. (1994). Each center point for SASW testing corresponds to the location of a CPT sounding taken during the UWL site investigation. Exact locations were determined utilizing a Garmin Fortrex 401 global positioning system accurate (95\%) from 15 to $30 \mathrm{ft}$. After determining a center point, pre-marked tape was laid with markings for each receiver spacing and source location (Figure 4.3). To mitigate the effects of background noise from UWL earthwork, receivers were oriented in-line with the direction of the background source. For points north of Labadie Bottom Road, testing was arrayed south to north and for points south of Labadie Bottom Road, west to east.

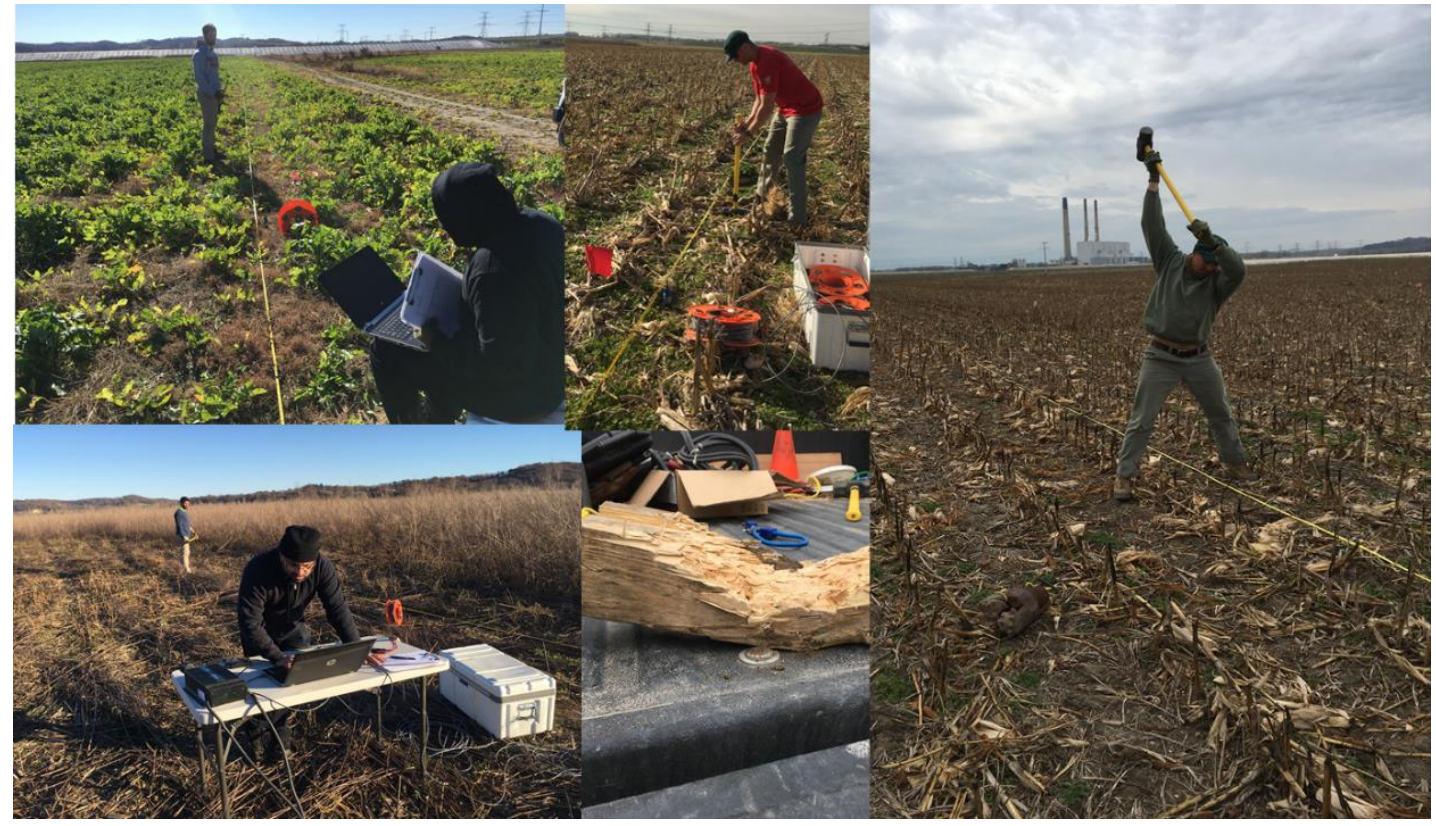

Figure 4.3 - SASW testing at Labadie UWL site, November 2016. 
A target depth of $40 \mathrm{ft}$ was chosen to coincide with CPT soundings typically taken to $36 \mathrm{ft}$. Receiver spacings of 5, 10, 20, and $40 \mathrm{ft}$ were utilized for all tests with the only exceptions being Points 66, 66A, and 64. At Points 66 and 64, soft topsoil coupled with high background noise (bulldozers working approximately 350 yards west) limited receiver spacing to $30 \mathrm{ft}$. At Point $66 \mathrm{~A}$, tests were done with receiver spacings of both 30 and $40 \mathrm{ft}$ to coincide with testing at Point 66.

Impulses were generated by impacting the 10-lb sledge hammer or 50-lb drop weight into blocks of wood seated firmly on the ground (Figure 4.3). For receiver spacings of 5 and $10 \mathrm{ft}$, the 10-lb sledge hammer generally produced sufficient energy when applied directly to the ground. Most tests at 20-ft receiver spacings required the wood block to effectively transfer energy from the hammer to the soil. At $40-\mathrm{ft}$ receiver spacings, nearly all tests required the 50-lb drop weight be dropped from 5 to $7 \mathrm{ft}$ onto the block of wood. Each site required careful selection of impulse trigger settings and responded differently to applied energy. While no specific observations were made regarding applied energy, it was generally noted that sites north of Labadie Bottom Road with firm silty topsoil required less energy, and those immediately south with mushy loam and tall vegetation required the most energy.

A minimum of three impulse signals were stacked for every test. For each receiver spacing, tests were deemed of sufficient quality when smooth wrapped phase diagrams were achieved across the first two full cycles of phase. If large jumps, significant skew, or low signal-to-noise ratios were observed, tests were restarted or additional impulses were stacked. Roughly half of the tests were completed with the minimum number of impulses and half required re-testing or additional stacking. 


\subsection{SASW Data Reduction and Dispersion Curve Fitting}

Data were reduced and dispersion curves fitted utilizing WinSASW Version 2.2. Text (.txt) files were exported from SignalCalc for each test point and receiver spacing combination and subsequently loaded into WinSASW. Interactive masking was performed for each test and the first $180^{\circ}$ of phase were generally disregarded (to avoid near field effects) while the three subsequent cycles of phase were used $\left(180^{\circ}\right.$ to $900^{\circ}$ unwrapped). Exceptions to these generalities were taken and more cycles of phase were used if phase diagrams displayed exceptionally low scatter. Similarly, phase diagrams were truncated within the targeted area if they exhibited unusual jumps or significant scatter.

Figure 4.4 shows selective masking of phase diagrams from Point 30. Shaded areas were discarded while unshaded areas were utilized to calculate the dispersion curve. At this location, for receiver spacings of 10 and $20 \mathrm{ft}$, the first three full cycles of phase were utilized and the rest discarded. For the 5-ft receiver spacing, an abnormality is present in the third cycle of phase resulting in a truncated target area. Similarly, the $40-\mathrm{ft}$ receiver spacing exhibits a large jump in the second cycle of phase causing subsequent cycles of phase to be discarded. 


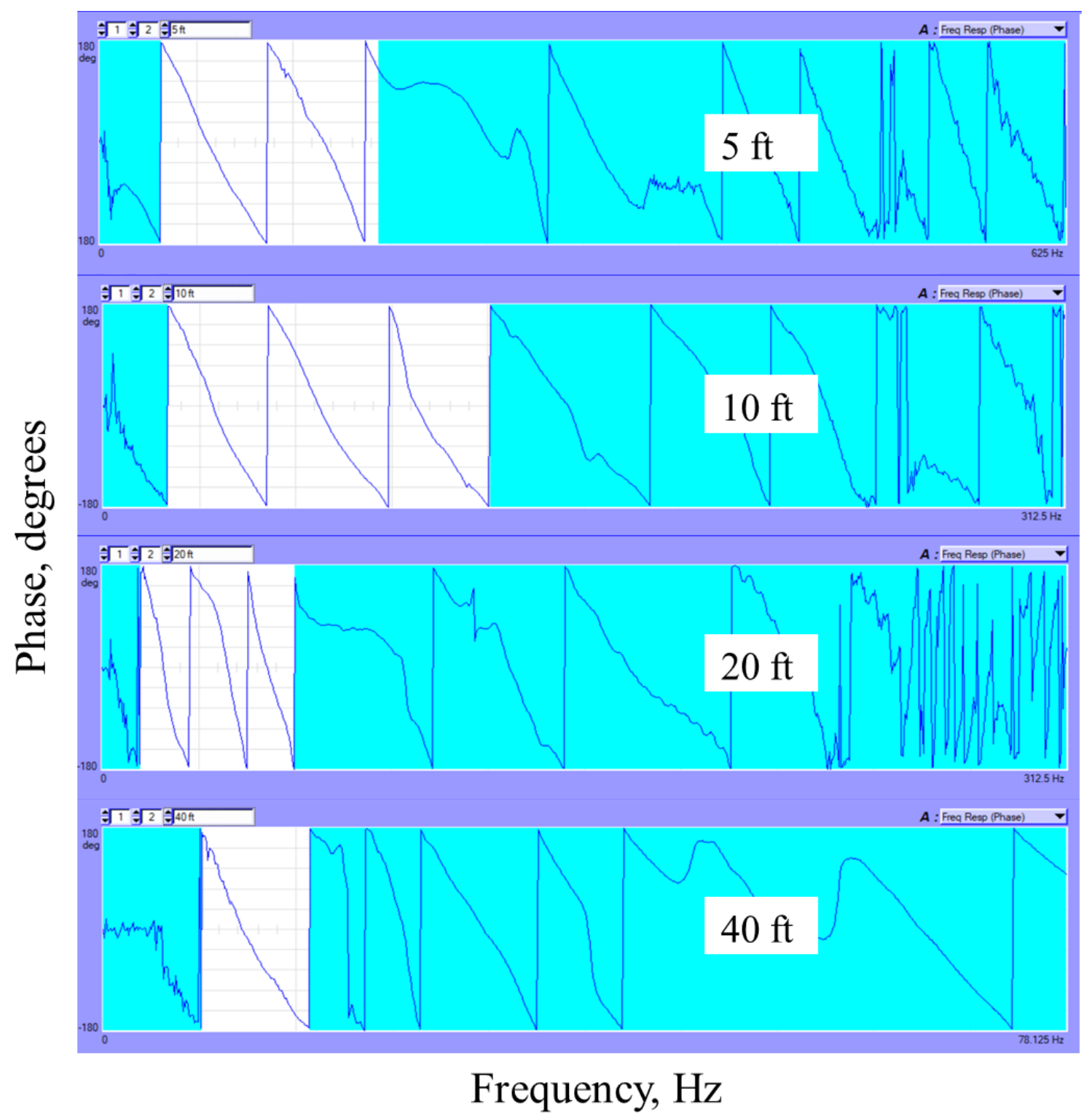

Figure 4.4 - WinSASW selective masking for Point 30. Vertical axis in degrees of phase (-180 to 180), horizontal axis in $\mathrm{Hz}$. 
Ц Representative Disp. Curve

Project: Project

General

GLOBAL Disp. Curve

Span Width: $\quad \sqrt[4]{40}$
No. of Segments: Domain: Freq $\square$ [III WaveL

Polynomial Order: $\sqrt[4]{4}$

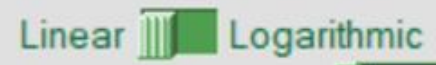

Increment Ratio: $\quad \hat{1.07}$

ARRAY Disp. Curve Polynomial Order. $\sqrt[4]{5}$

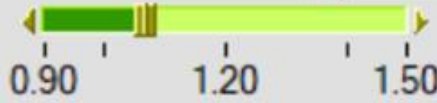
for $5 \mathrm{ft}[1: 2]$

$\times$
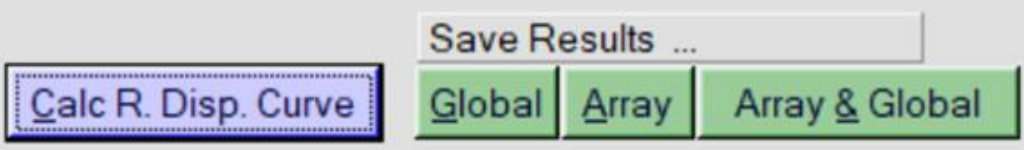

Close

Figure 4.5 - WinSASW 2.2 representative dispersion curve settings.

Global dispersion curves were fitted according to wavelength and phase velocity utilizing default settings in WinSASW. The only exception to default settings is that dispersion curves were calculated in the wavelength domain (Figure 4.5). Figure 4.6 shows the composite measured dispersion curve for Point 30 consisting of individual dispersion curves for each receiver spacing ( $5 \mathrm{ft}, 10 \mathrm{ft}, 20 \mathrm{ft}, 40 \mathrm{ft}$ ). The global dispersion curve for Point 30 is shown in Figure 4.7. 


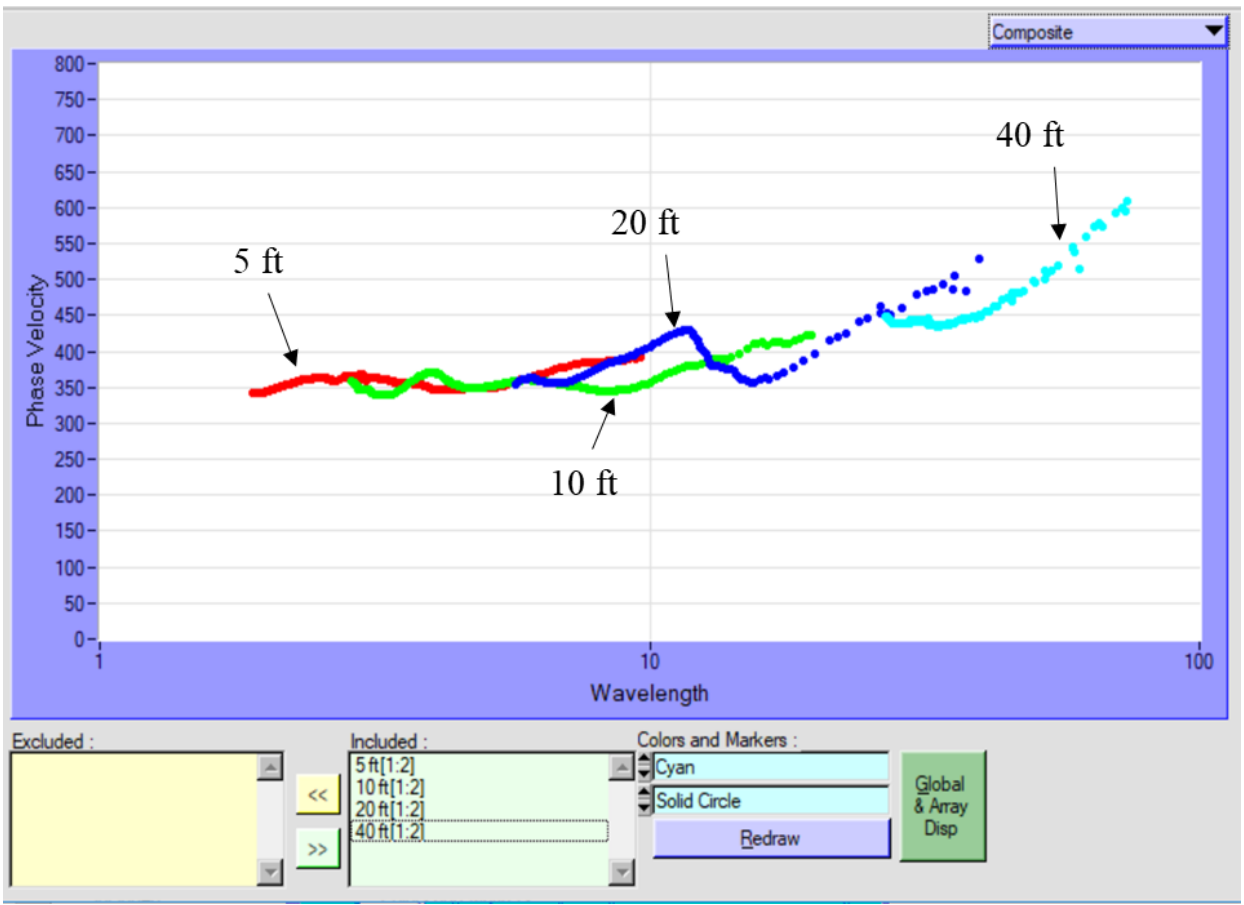

Figure 4.6 - Point 30 composite dispersion curve with associated receiver spacings.

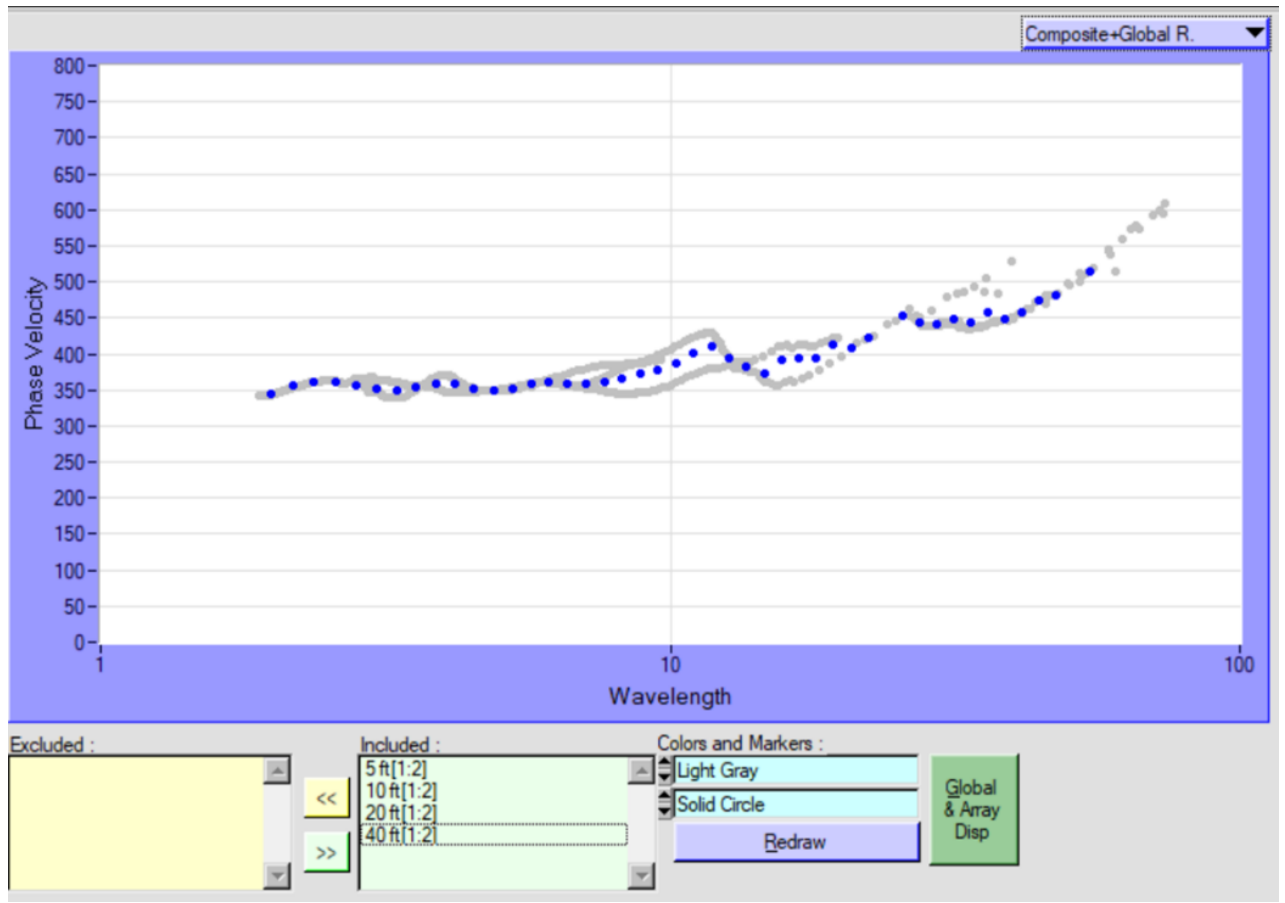

Figure 4.7 - Point 30 global dispersion curve (blue dots). 
Finally, global dispersion curves were occasionally calculated with missing receiver spacings. This was only possible if the remaining segments sufficiently overlapped (Figure 4.8). All global dispersion curves were exported from WinSASW as text files containing phase velocity $\left(V_{p h}\right)$, wavelength $(\lambda)$, and frequency $(f)$ for further processing in Microsoft Excel.

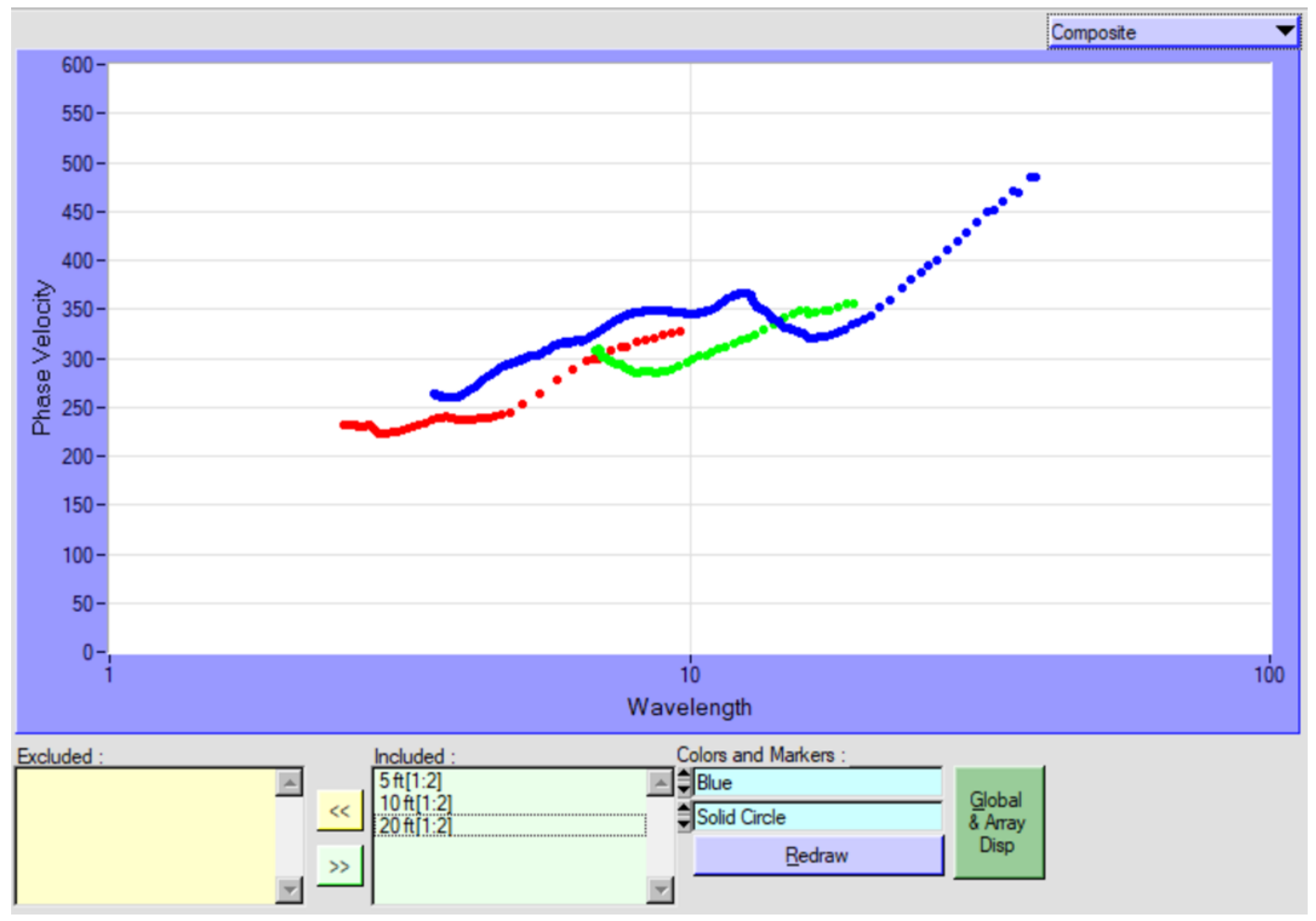

Figure 4.8 - Point 28 composite dispersion curve (no 40-ft receiver spacing).

\subsection{Quantifying variability}

Two approaches were utilized to quantify and compare variability in CPT and SASW data. The first method will be referred to as the "preliminary method" and the second the "equivalent wavelength method" (EWM). The preliminary method is a straightforward approach that was intended as a starting point for the development of a more refined 
method. The EWM was developed only after the limitations of the preliminary method were realized through data analysis. In essence, the EWM incorporates the same framework as the preliminary method but improves it by weighting CPT data as a function of Rayleigh wave energy with depth. In doing so, the EWM ensures that an equitable comparison is made between measurements taken across approximately the same depths.

\subsubsection{Preliminary Method}

The preliminary method quantifies variability by calculating and averaging COVs of SASW and CPT data. Recall from Section 4.4 that measured SASW dispersion curves consist of phase velocity $\left(V_{p h}\right)$ and their corresponding wavelength $(\lambda)$. Put simply, the preliminary method quantifies variability of dispersion curves by calculating COVs amongst $V_{p h}$ for the same $\lambda$ measured at several test points within a group. A challenge in utilizing this method is that in WinSASW, dispersion curves are not calculated at constant

$\lambda$. In order to compare $V_{p h}$ at the same $\lambda$ for each dispersion curve, $V_{p h}$ must therefore be interpolated for desired values of $\lambda$. For the preliminary method, $V_{p h}$ was interpolated every $0.5 \mathrm{ft}$ for $\lambda$ less than $10 \mathrm{ft}$, every $1 \mathrm{ft}$ for $\lambda$ between 10 and $20 \mathrm{ft}$, and every $5 \mathrm{ft}$ thereafter (Figure 4.9, Table 4.2). 


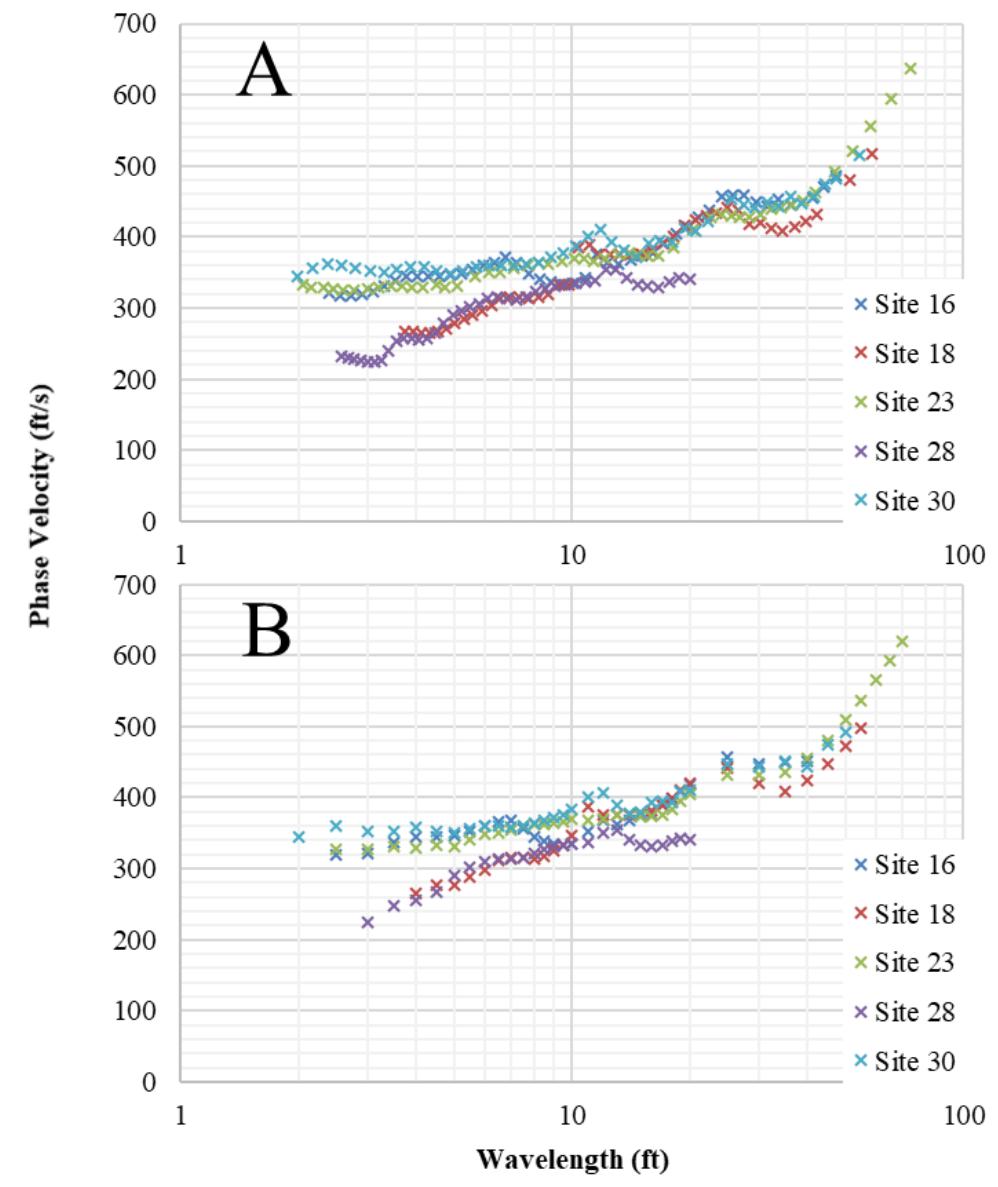

Figure 4.9 - Group 23 global dispersion curves A.) Direct from WinSASW B.) Interpolated for same wavelengths.

Once all $V_{p h}$ values have been interpolated for every test point within a group, COVs are calculated for each $\lambda$ (Table 4.2). However, because not all dispersion curves yield $V_{p h}$ over the same range of $\lambda$, cases exist where $V_{p h}$ for a particular $\lambda$ are not available at every test point within a group. In such cases, COVs are calculated if three or more $V_{p h}$ are available for any particular $\lambda$. This concept is illustrated in Table 4.2 where COVs are calculated when three or more $V_{p h}$ values are available ( $\lambda$ ranging from 2.5 to $50 \mathrm{ft}$ ). Finally, COVs for each $\lambda$ are averaged to provide the overall mean COV for the group (Table 4.2). 
Table 4.2 - Group 23 dispersion curve variability calculation.

\begin{tabular}{|c|c|c|c|c|c|c|}
\hline$\lambda(\mathrm{ft})$ & $\begin{array}{c}\text { Point } 16 \\
V_{p h}(\mathrm{ft} / \mathrm{s})\end{array}$ & $\begin{array}{c}\text { Point } 18 \\
V_{p h}(\mathrm{ft} / \mathrm{s})\end{array}$ & $\begin{array}{c}\text { Point } 23 \\
V_{p h}(\mathrm{ft} / \mathrm{s})\end{array}$ & $\begin{array}{c}\text { Point } 28 \\
V_{p h}(\mathrm{ft} / \mathrm{s})\end{array}$ & $\begin{array}{c}\text { Point 30 } \\
V_{p h}(\mathrm{ft} / \mathrm{s})\end{array}$ & COV $V_{p h}$ \\
\hline 2.0 & & & & & 345 & \\
\hline 2.5 & 320 & & 327 & & 361 & 0.065 \\
\hline 3.0 & 321 & & 328 & 224 & 353 & 0.184 \\
\hline 3.5 & 337 & & 330 & 248 & 353 & 0.149 \\
\hline 4.0 & 345 & 266 & 329 & 256 & 358 & 0.151 \\
\hline 4.5 & 345 & 278 & 332 & 267 & 353 & 0.126 \\
\hline 5.0 & 348 & 278 & 330 & 291 & 350 & 0.104 \\
\hline 5.5 & 353 & 288 & 341 & 302 & 355 & 0.094 \\
\hline 6.0 & 360 & 299 & 349 & 310 & 360 & 0.086 \\
\hline 6.5 & 367 & 312 & 351 & 314 & 360 & 0.076 \\
\hline 7.0 & 368 & 316 & 355 & 313 & 359 & 0.075 \\
\hline 7.5 & 356 & 315 & 359 & 315 & 361 & 0.071 \\
\hline 8.0 & 344 & 314 & 363 & 322 & 363 & 0.066 \\
\hline 8.5 & 339 & 317 & 363 & 328 & 368 & 0.064 \\
\hline 9.0 & 336 & 325 & 364 & 331 & 373 & 0.061 \\
\hline 9.5 & 332 & 332 & 367 & 333 & 376 & 0.062 \\
\hline 10.0 & 334 & 347 & 370 & 335 & 383 & 0.062 \\
\hline 11.0 & 353 & 388 & 368 & 338 & 402 & 0.070 \\
\hline 12.0 & 369 & 376 & 369 & 350 & 406 & 0.055 \\
\hline 13.0 & 363 & 376 & 375 & 354 & 389 & 0.036 \\
\hline 14.0 & 368 & 376 & 377 & 340 & 378 & 0.043 \\
\hline 15.0 & 373 & 375 & 374 & 333 & 379 & 0.053 \\
\hline 16.0 & 376 & 382 & 373 & 331 & 392 & 0.063 \\
\hline 17.0 & 388 & 390 & 377 & 332 & 394 & 0.068 \\
\hline 18.0 & 400 & 399 & 384 & 339 & 393 & 0.066 \\
\hline 19.0 & 409 & 411 & 394 & 343 & 408 & 0.073 \\
\hline 20.0 & 418 & 419 & 405 & 340 & 411 & 0.083 \\
\hline 25.0 & 458 & 442 & 431 & & 447 & 0.025 \\
\hline 30.0 & 447 & 420 & 432 & & 444 & 0.029 \\
\hline 35.0 & 449 & 409 & 435 & & 451 & 0.044 \\
\hline 40.0 & 452 & 424 & 455 & & 443 & 0.032 \\
\hline 45.0 & & 447 & 481 & & 475 & 0.039 \\
\hline 50.0 & & 473 & 509 & & 493 & 0.036 \\
\hline 55.0 & & 498 & 537 & & & \\
\hline 60.0 & & & 565 & & & \\
\hline 65.0 & & & 592 & & & \\
\hline 70.0 & & & 619 & & & \\
\hline Avg & - & - & - & & & \\
\hline & & & & & & \\
\hline
\end{tabular}


The preliminary method compares CPT data in the same manner as SASW dispersion curves, with depth (z) replacing $\lambda$, and $q_{t}$ or $f_{s}$ replacing $V_{p h}$ (Table 4.3). For each group of CPT soundings, a COV is calculated from $q_{t}$ or $f_{s}$ at a given depth. For depths where less than three measurements are available, no COV is calculated. Coefficients of variation are then averaged across the range of available depths to provide the overall mean COV of $q_{t}$ or $f_{s}$ for the group. The mean COV of CPT data may then be compared to the mean COV of $V_{p h}$ from dispersion curves measured at the same physical locations.

Table 4.3 - Group 23 CPT $q_{t}$ variability calculations.

\begin{tabular}{|c|c|c|c|c|c|c|}
\hline \multicolumn{7}{|c|}{ Group 23 (16,18,23,28,30) } \\
\hline \multirow{2}{*}{ Depth (ft) } & $\begin{array}{c}\text { Point } \\
16 q_{t} \\
(\mathrm{psi})\end{array}$ & $\begin{array}{c}\text { Point } \\
18 q_{t} \\
(\mathrm{psi})\end{array}$ & $\begin{array}{c}\text { Point } 23 q_{t} \\
(\mathrm{psi})\end{array}$ & $\begin{array}{c}\text { Point } \\
28 q_{t} \\
(\mathrm{psi})\end{array}$ & $\begin{array}{c}\text { Point } \\
30 q_{t} \\
(\mathrm{psi})\end{array}$ & COV $q_{t}$ \\
\hline 1.25 & 266 & 148 & 150 & 265 & 136 & 0.34 \\
\hline 3.75 & 207 & 194 & 215 & 174 & 177 & 0.09 \\
\hline 6.25 & 281 & 179 & 367 & 155 & 299 & 0.34 \\
\hline 8.75 & 384 & 610 & 314 & 333 & 943 & 0.51 \\
\hline 11.25 & 368 & 571 & 770 & 394 & 1513 & 0.65 \\
\hline 13.75 & 762 & 1191 & 1279 & 169 & 2495 & 0.73 \\
\hline 16.25 & 1007 & 1189 & 1201 & 972 & 693 & 0.20 \\
\hline 18.75 & 1272 & 826 & 552 & 1208 & 1321 & 0.32 \\
\hline 21.25 & 1768 & 776 & 1567 & 1242 & 1441 & 0.28 \\
\hline 23.75 & 2664 & 1404 & 2454 & 1803 & 1617 & 0.27 \\
\hline 26.25 & 1865 & 2413 & 2387 & 1835 & 2550 & 0.15 \\
\hline 28.75 & 2898 & 3011 & 1518 & 1838 & 2323 & 0.28 \\
\hline 31.25 & 2463 & 1603 & 2953 & 1591 & 2085 & 0.27 \\
\hline 33.75 & 4296 & 2457 & 2775 & 2679 & 1270 & 0.40 \\
\hline 36.25 & 4684 & 1948 & 4116 & 1496 & 2568 & 0.47 \\
\hline Avg & - & - & - & - & - & $\mathbf{0 . 3 5}$ \\
\hline
\end{tabular}




\subsubsection{Equivalent Wavelength Method (EWM)}

The second method utilized to compare CPT and SASW data was developed based on the shortcomings of the preliminary method. An in-depth discussion of those shortcomings and the need to enhance the preliminary method is provided in Chapter 6 . The equivalent wavelength method $(\mathrm{EWM})$ utilizes much of the same procedure as the preliminary method, with the following improvements:

1. CPT data $\left(q_{t}, f_{s}\right)$ are weighted proportionally to Rayleigh wave energy distribution as a function of depth.

2. The same number of CPT and SASW measurements, taken across the same depths, are compared.

The principle behind the first improvement lies in the way Rayleigh wave energy dissipates exponentially with depth (Figure 4.10). This phenomenon causes the stiffness of upper soil layers to have a larger effect on Rayleigh wave propagation than stiffness of lower layers. For example, in a Rayleigh wave with $\lambda_{R}=10 \mathrm{ft}$, the overwhelming majority of wave energy (more than $70 \%$ ) propagates through the upper $5 \mathrm{ft}$ of soil. A simple approximation for quantifying the weight or percent of Rayleigh wave energy bounded between any two depths for a given $\lambda$ is given below from Leong and Aung (2012).

$$
\begin{aligned}
& W_{i}=\frac{\int_{Z_{i-1}}^{Z_{i}} f\left(\frac{z}{\lambda}\right) d z}{\int_{0}^{\lambda} f\left(\frac{z}{\lambda}\right) d z} \\
& f\left(\frac{z}{\lambda}\right)=1-(z / \lambda)^{3 / 2}
\end{aligned}
$$


Where $Z_{i-1}$ is the depth at the top of layer $i$, and $Z_{i}$ is the depth at the bottom of layer $i$ for a particular $\lambda$. For example, the weight $W_{i}$ (or percent) of Rayleigh wave energy transmitted between 2.5 and $5 \mathrm{ft}$ below the surface for $\lambda_{R}=10 \mathrm{ft}$ is calculated as follows:

$$
\begin{gathered}
W_{i}=\frac{\int_{Z_{i-1}}^{Z_{i}} f\left(\frac{Z}{\lambda}\right) d z}{\int_{0}^{\lambda} f\left(\frac{Z}{\lambda}\right) d z}=\frac{\int_{Z_{i-1}}^{Z_{i}}\left[1-\left(\frac{Z}{\lambda}\right)^{\frac{3}{2}}\right] d z}{\int_{0}^{\lambda}\left[1-\left(\frac{Z}{\lambda}\right)^{\frac{3}{2}}\right] d z} \\
=\frac{\left[Z_{i}-(2 / 5) Z_{i}\left(\frac{Z_{i}}{\lambda}\right)^{3 / 2}\right]-\left[Z_{i-1}-(2 / 5) Z_{i-1}\left(\frac{Z_{i-1}}{\lambda}\right)^{3 / 2}\right]}{\lambda-\frac{2}{5} \lambda}= \\
\frac{\left[5-(2 / 5) 5\left(\frac{5}{10}\right)^{3 / 2}\right]-\left[2.5-\left(\frac{2}{5}\right) 2.5\left(\frac{2.5}{10}\right)^{3 / 2}\right]}{10-\frac{2}{5} 10}=0.32 \text { or } 32 \%
\end{gathered}
$$

This simple energy approximation can be used to weight CPT data with depth in approximately the same manner that Rayleigh wave energy is distributed. To achieve such an approximation, several "equivalent wavelengths" $\left(\lambda_{e q}\right)$ and corresponding CPT measurements $\left(\mathrm{CPT}_{e q}\right)$ consisting of the equivalent corrected tip resistance $\left(q_{\text {teq }}\right)$ or the equivalent skin friction $\left(f_{\text {seq }}\right)$ are calculated for each site. $\mathrm{CPT}_{e q}$ is calculated by summing the product of each CPT measurement $\left(q_{t}\right.$ or $\left.f_{s}\right)$ for a given depth and its corresponding weight $\left(W_{i}\right)$ (Eq. 4.3). Thus, each CPT sounding is converted into several $\mathrm{CPT}_{e q}$ measurements $\left(q_{t e q}, f_{\text {seq }}\right)$ each corresponding to a particular $\lambda_{\text {eq. }}$. Finally, variability in $q_{\text {teq }}$ or $f_{s e q}$ for each $\lambda_{e q}$ may be compared in the same manner that variability in $V_{p h}$ is compared for a specific $\lambda$ in the preliminary method. 


$$
C P T_{e q\left(\lambda_{e q}\right)}=\Sigma\left(C P T_{i} \cdot W_{i\left(\lambda_{e q}\right)}\right)
$$

Table 4.3 shows the values of $W_{i}$ applied to $\mathrm{CPT}_{i}$ in calculating $\mathrm{CPT}_{e q}$ for a particular $\lambda_{e q}$. For example, $\mathrm{CPT}_{e q}$ for $\lambda_{e q}=2.5 \mathrm{ft}$ is calculated by multiplying the $\mathrm{CPT}$ measurement taken at $1.25 \mathrm{ft}$ (center of interval from 0 to $2.5 \mathrm{ft}$ ) by 1.0. Similarly, $\mathrm{CPT}_{e q}$ for $\lambda_{e q}=5 \mathrm{ft}$, is calculated by summing the CPT measurement at $1.25 \mathrm{ft}$ multiplied by 0.715 , and the CPT measurement at $3.75 \mathrm{ft}$ multiplied by 0.285 . This process is repeated and $\mathrm{CPT}_{e q}$ is calculated for as many as $15 \lambda_{e q}$ depending on the depth of the CPT sounding.

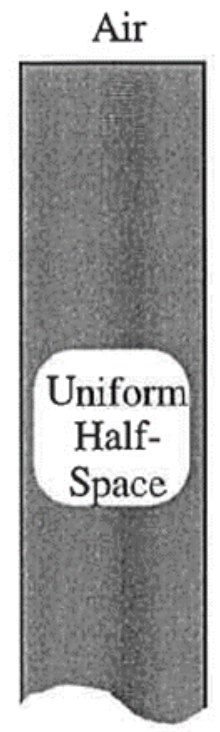

a) Material
Profile

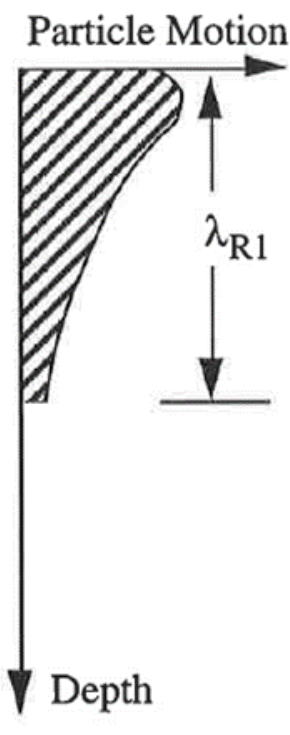

b) Shorter Wavelength, $\lambda_{\mathrm{R} 1}$

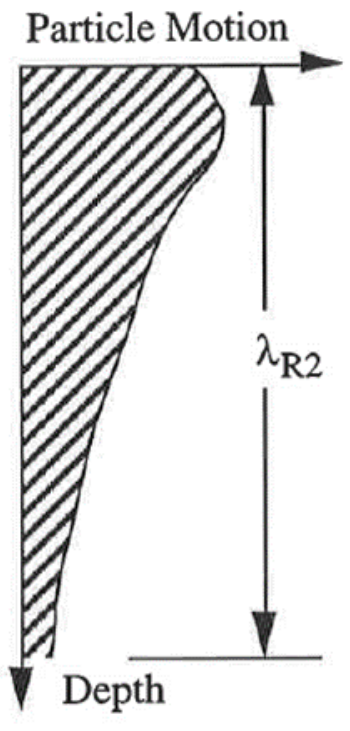

c) Longer Wavelength, $\lambda_{\mathrm{R} 2}$

Figure 4.10 - Approximate distribution of vertical particle motion with depth for different length Rayleigh waves (from Stokoe et al. 1994). 


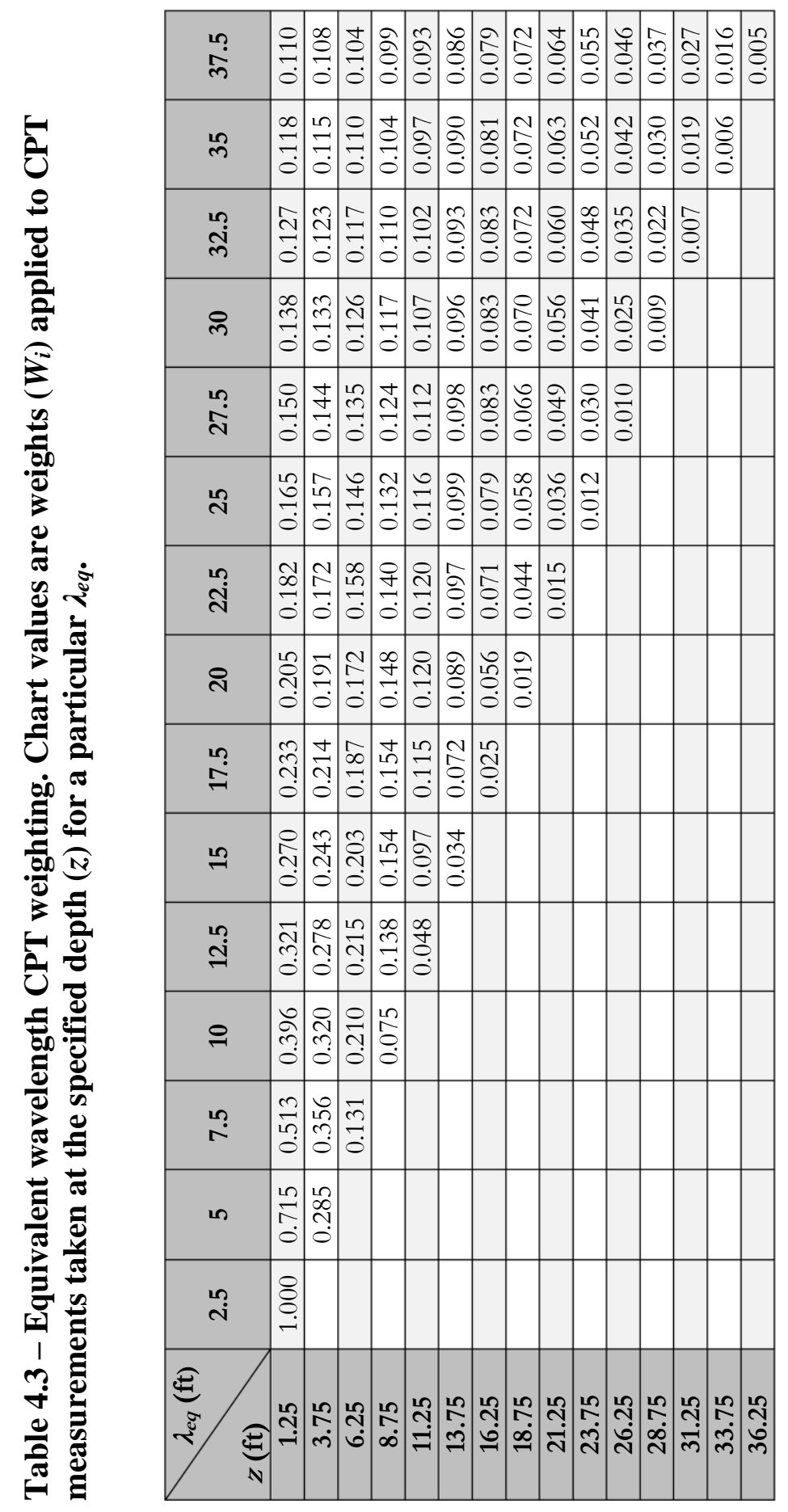


Finally, the second improvement to the preliminary method is implemented in the EWM by comparing variability in $\mathrm{CPT}_{e q}$ to variability in $V_{p h}$ across the same $\lambda$ or $\lambda_{e q}$. That is, for every calculated $\mathrm{CPT}_{e q}$ at a specific location, $V_{p h}$ for the same $\lambda$ is interpolated from SASW data. For situations where either $\mathrm{CPT}_{e q}$ or $V_{p h}$ for the same $\lambda$ are unavailable due to a lack of CPT or SASW data, no comparison is made.

\subsubsection{EWM Example Calculation}

Recall that Group 23 consists of Points 16, 18, 23, 28, and 30, and that each point represents a specific surface location where a CPT sounding and SASW test have been conducted. First, after ensuring that CPT and SASW data are available for all points in Group 23, $q_{\text {teq }}$ is calculated for $\lambda_{e q}$ ranging from 2.5 to $37.5 \mathrm{ft}$ on a 2.5 -ft interval. Table 4.4 shows $q_{\text {teq }}$ calculations for Point 16 and Table 4.5 shows $q_{\text {teq }}$ calculations for all points in Group 23. Once all $q_{\text {teq }}$ values are calculated, a COV for each $\lambda_{e q}$ may be determined. In the case of Group 23, the COV of $q_{t e q}$ is calculated for all $15 \lambda_{e q}$ because $q_{t e q}$ is available for three or more points for all $\lambda_{e q}$. However, final COVs and the overall mean COV for the group cannot be calculated until the availability of $V_{p h}$ from SASW testing is known. 
Table 4.4 - EWM calculation of $\boldsymbol{q}_{t e q}$ for Point 16.

\begin{tabular}{|c|c|c|c|c|c|c|}
\hline \multirow[b]{2}{*}{$\begin{array}{c}\text { Depth } \\
z \text { (ft) }\end{array}$} & \multicolumn{3}{|c|}{ Measured CPT data } & \multicolumn{3}{|c|}{ EWM } \\
\hline & $\begin{array}{c}q_{t} \\
(\mathbf{p s i})\end{array}$ & $\begin{array}{c}f_{s} \\
(\mathrm{psi})\end{array}$ & $\underset{(p s i)}{u}$ & $\begin{array}{l}\lambda_{e q} \\
(\mathbf{f t})\end{array}$ & $\begin{array}{c}\boldsymbol{q}_{\text {teq }} \\
(\mathbf{p s i})\end{array}$ & $\begin{array}{c}f_{\text {seq }} \\
(\mathrm{psi})\end{array}$ \\
\hline 1.25 & 266 & 2.9 & -0.8 & 2.5 & 266 & 2.9 \\
\hline 3.75 & 207 & 4.6 & -1.3 & 5.0 & 249 & 3.4 \\
\hline 6.25 & 281 & 3.8 & -2.0 & 7.5 & 247 & 3.6 \\
\hline 8.75 & 384 & 2.8 & -2.7 & 10.0 & 259 & 3.6 \\
\hline 11.25 & 368 & 2.9 & -3.5 & 12.5 & 274 & 3.6 \\
\hline 13.75 & 762 & 3.9 & -0.4 & 15.0 & 299 & 3.5 \\
\hline 16.25 & 1007 & 6.8 & 1.3 & 17.5 & 340 & 3.6 \\
\hline 18.75 & 1272 & 5.9 & 3.5 & 20.0 & 392 & 3.7 \\
\hline 21.25 & 1768 & 9.9 & 3.4 & 22.5 & 455 & 3.9 \\
\hline 23.75 & 2664 & 10.0 & 5.0 & 25.0 & 536 & 4.2 \\
\hline 26.25 & 1865 & 5.5 & 6.5 & 27.5 & 623 & 4.5 \\
\hline 28.75 & 2898 & 10.0 & 7.3 & 30.0 & 710 & 4.7 \\
\hline 31.25 & 2463 & 5.8 & 8.7 & 32.5 & 799 & 4.9 \\
\hline 33.75 & 4296 & 12.6 & 9.3 & 35.0 & 894 & 5.1 \\
\hline 36.25 & 4684 & 17.0 & 10.0 & 37.5 & 1002 & 5.3 \\
\hline
\end{tabular}

Table 4.5 - EWM calculation of $q_{t e q}$ for Group 23 .

\begin{tabular}{|c|c|c|c|c|c|c|}
\hline$\lambda_{\text {eq }}(\mathbf{f t})$ & $\begin{array}{c}\text { Point 16 } \\
\boldsymbol{q}_{\text {teq }}(\mathbf{p s i})\end{array}$ & $\begin{array}{c}\text { Point 18 } \\
\boldsymbol{q}_{\text {teq }}(\mathbf{p s i})\end{array}$ & $\begin{array}{c}\text { Point 23 } \\
\boldsymbol{q}_{\text {teq }}(\mathbf{p s i})\end{array}$ & $\begin{array}{c}\text { Point 28 } \\
\boldsymbol{q}_{\text {teq }}(\mathbf{p s i})\end{array}$ & $\begin{array}{c}\text { Point 30 } \\
\boldsymbol{q}_{\text {teq }}(\mathbf{p s i})\end{array}$ & $\begin{array}{c}\text { COV } \\
\boldsymbol{q}_{\text {teq }}\end{array}$ \\
\hline 2.5 & 266 & 148 & 150 & 265 & 136 & 0.39 \\
\hline 5.0 & 249 & 161 & 168 & 239 & 148 & 0.25 \\
\hline 7.5 & 247 & 168 & 201 & 218 & 172 & 0.16 \\
\hline 10.0 & 259 & 204 & 228 & 218 & 244 & 0.09 \\
\hline 12.5 & 274 & 252 & 267 & 232 & 360 & 0.18 \\
\hline 15.0 & 299 & 313 & 333 & 240 & 516 & 0.31 \\
\hline 17.5 & 340 & 385 & 409 & 261 & 642 & 0.35 \\
\hline 20.0 & 392 & 446 & 466 & 303 & 722 & 0.34 \\
\hline 22.5 & 455 & 491 & 516 & 355 & 787 & 0.31 \\
\hline 25.0 & 536 & 533 & 583 & 414 & 844 & 0.24 \\
\hline 27.5 & 623 & 588 & 663 & 481 & 906 & 0.21 \\
\hline 30.0 & 710 & 660 & 737 & 548 & 973 & 0.18 \\
\hline 32.5 & 799 & 733 & 811 & 611 & 1036 & 0.16 \\
\hline 35.0 & 894 & 802 & 888 & 674 & 1089 & 0.13 \\
\hline 37.5 & 1002 & 866 & 972 & 736 & 1137 & 0.11 \\
\hline Avg & -- & -- & -- & -- & - & $\mathbf{0 . 2 3}$ \\
\hline
\end{tabular}


Next, $V_{p h}$ values are interpolated from SASW dispersion curves for the same $\lambda$ utilized in calculating $q_{t e q}$ (Table 4.6). Once $V_{p h}$ values have been interpolated for all points, they are compared to available $q_{\text {teq }}$ from CPT testing. Table 4.7 shows that in the case of Group 23, $V_{p h}$ values are missing at $\lambda$ of $2.5 \mathrm{ft}$ for Point 18 , as well as $\lambda$ of $2.5 \mathrm{ft}$ and 22.5 to 37.5 $\mathrm{ft}$ for Point 28. Therefore, mean COV calculations for both $q_{\text {teq }}$ and $V_{p h}$ will exclude COVs for $\lambda$ of $2.5 \mathrm{ft}$ at Point 18 , and $\lambda$ of $2.5 \mathrm{ft}$ and 22.5 to $37.5 \mathrm{ft}$ at Point 28 (Table 4.8).

Table 4.6 - Interpolation of target $V_{p h}$ values for Point 16 .

\begin{tabular}{|c|c|c|c|c|c|c|c|c|c|}
\hline \multicolumn{3}{|c|}{$\begin{array}{c}\text { Raw Dispersion } \\
\text { Data } \\
\end{array}$} & \multicolumn{2}{|c|}{ Interpolation } & \multicolumn{3}{|c|}{$\begin{array}{c}\text { Raw Dispersion } \\
\text { Data } \\
\end{array}$} & \multicolumn{2}{|c|}{ Interpolation } \\
\hline $\begin{array}{c}V_{p h} \\
(\mathbf{f t} / \mathbf{s})\end{array}$ & $\underset{(\mathbf{H z})}{f}$ & $\lambda(\mathbf{f t})$ & $\begin{array}{c}\lambda \\
\text { target } \\
(\mathbf{f t})\end{array}$ & $\begin{array}{c}V_{p h} \\
(\lambda \text { target }) \\
(\mathbf{f t} / \mathbf{s})\end{array}$ & $\begin{array}{c}V_{p h} \\
(\mathbf{f t} / \mathbf{s})\end{array}$ & $\underset{(\mathbf{H z})}{f}$ & $\lambda(\mathbf{f t})$ & $\begin{array}{c}\lambda \\
\text { target } \\
\text { (ft) }\end{array}$ & $\begin{array}{c}V_{p h} \\
(\lambda \text { target }) \\
(\mathbf{f t} / \mathbf{s})\end{array}$ \\
\hline 322 & 134.0 & 2.4 & & & 343 & 31.8 & 10.8 & & \\
\hline 318 & 124.4 & 2.6 & 2.5 & 320 & 376 & 32.6 & 11.5 & & \\
\hline 317 & 116.4 & 2.7 & & & 363 & 29.5 & 12.3 & & \\
\hline 319 & 109.8 & 2.9 & & & 363 & 27.5 & 13.2 & 12.5 & 363 \\
\hline 323 & 104.3 & 3.1 & & & 368 & 26.1 & 14.1 & & \\
\hline 330 & 99.9 & 3.3 & & & 374 & 24.8 & 15.1 & 15.0 & 373 \\
\hline 338 & 95.9 & 3.5 & & & 377 & 23.4 & 16.1 & & \\
\hline 344 & 91.5 & 3.8 & & & 392 & 22.7 & 17.2 & & \\
\hline 345 & 86.1 & 4.0 & & & 404 & 21.9 & 18.4 & 17.5 & 394 \\
\hline 345 & 80.6 & 4.3 & & & 415 & 21.1 & 19.7 & & \\
\hline 345 & 75.6 & 4.6 & & & 427 & 20.3 & 21.1 & 20.0 & 418 \\
\hline 347 & 71.1 & 4.9 & & & 437 & 19.4 & 22.5 & 22.5 & 437 \\
\hline 349 & 67.0 & 5.2 & 5.0 & 348 & 456 & 18.9 & 24.1 & & \\
\hline 354 & 63.6 & 5.6 & & & 459 & 17.8 & 25.8 & 25.0 & 458 \\
\hline 360 & 60.5 & 5.9 & & & 459 & 16.6 & 27.6 & 27.5 & 459 \\
\hline 365 & 57.4 & 6.3 & & & 449 & 15.2 & 29.5 & & \\
\hline 371 & 54.7 & 6.8 & & & 443 & 14.0 & 31.5 & 30.0 & 447 \\
\hline 365 & 50.3 & 7.2 & & & 453 & 13.4 & 33.7 & 32.5 & 447 \\
\hline 349 & 45.0 & 7.7 & 7.5 & 356 & 445 & 12.3 & 36.1 & 35.0 & 449 \\
\hline 340 & 41.1 & 8.3 & & & 454 & 11.0 & 41.3 & 37.5 & 447 \\
\hline 337 & 38.1 & 8.8 & & & 470 & 10.6 & 44.2 & & \\
\hline 332 & 35.2 & 9.4 & & & 485 & 10.3 & 47.3 & & \\
\hline 335 & 33.2 & 10.1 & 10.0 & 334 & -- & -- & -- & -- & -- \\
\hline
\end{tabular}


Table 4.7 - Variability of $V_{p h}$ at selected $\lambda$ for Group 23.

\begin{tabular}{|c|c|c|c|c|c|c|}
\hline $\boldsymbol{\lambda}$ (ft) & $\begin{array}{c}\text { Point } \\
\mathbf{1 6} \boldsymbol{V}_{\boldsymbol{p h}} \\
(\mathbf{f t} / \mathbf{s})\end{array}$ & $\begin{array}{c}\text { Point } \\
\mathbf{1 8} \boldsymbol{V}_{\boldsymbol{p h}} \\
(\mathbf{f t} / \mathbf{s})\end{array}$ & $\begin{array}{c}\text { Point } \\
\mathbf{2 3} \boldsymbol{V}_{\boldsymbol{p h}} \\
(\mathbf{f t} / \mathbf{s})\end{array}$ & $\begin{array}{c}\text { Point } \\
\mathbf{2 8} \boldsymbol{V}_{\boldsymbol{p h}} \\
(\mathbf{f t} / \mathbf{s})\end{array}$ & $\begin{array}{c}\text { Point } \\
\mathbf{3 0} \boldsymbol{V}_{\boldsymbol{p h}} \\
(\mathbf{f t} / \mathbf{s})\end{array}$ & $\begin{array}{c}\mathbf{C O V} \\
\boldsymbol{V}_{\boldsymbol{p h}}\end{array}$ \\
\hline 2.5 & 320 & & 327 & & 361 & 0.065 \\
\hline 5.0 & 348 & 278 & 330 & 291 & 350 & 0.104 \\
\hline 7.5 & 356 & 315 & 359 & 315 & 361 & 0.071 \\
\hline 10.0 & 334 & 347 & 370 & 335 & 383 & 0.062 \\
\hline 12.5 & 363 & 376 & 372 & 355 & 395 & 0.041 \\
\hline 15.0 & 373 & 375 & 374 & 333 & 379 & 0.053 \\
\hline 17.5 & 394 & 394 & 379 & 337 & 394 & 0.066 \\
\hline 20.0 & 418 & 419 & 405 & 340 & 411 & 0.083 \\
\hline 22.5 & 437 & 431 & 427 & & 425 & 0.013 \\
\hline 25.0 & 458 & 442 & 431 & & 447 & 0.025 \\
\hline 27.5 & 459 & 429 & 428 & & 444 & 0.033 \\
\hline 30.0 & 447 & 420 & 432 & & 444 & 0.029 \\
\hline 32.5 & 447 & 412 & 439 & & 446 & 0.038 \\
\hline 35.0 & 449 & 409 & 435 & & 451 & 0.044 \\
\hline 37.5 & 447 & 416 & 448 & & 452 & 0.038 \\
\hline Avg & -- & -- & -- & -- & -- & $\mathbf{0 . 0 5 1}$ \\
\hline
\end{tabular}

Finally, after COV calculations are made for available $\lambda$ where three or more values are present in each case, the overall mean COV of $q_{t e q}$ and $V_{p h}$ is calculated for the group. For Group 23, the mean COV of $V_{p h}$ is 0.051 (Table 4.7) and the mean COV of $q_{\text {teq }}$ for the same $\lambda$ is 0.23 (Table 4.8). As previously stated, this example excludes some $q_{\text {teq }}$ measurements because no corresponding $V_{p h}$ is available. It should be noted, however, that cases also exist where $V_{p h}$ values are not utilized because no corresponding $q_{t e q}$ is available. 
Table 4.8 - Variability of $q_{t e q}$ at selected $\lambda_{e q}$ for Group 23. Struck through values not included in $\mathrm{COV} q_{\text {teq }}$ calculation.

\begin{tabular}{|c|c|c|c|c|c|c|}
\hline$\lambda_{\text {eq }}$ (ft) & $\begin{array}{c}\text { Point 16 } \\
\boldsymbol{q}_{\text {teq }}(\mathbf{p s i})\end{array}$ & $\begin{array}{c}\text { Point 18 } \\
\boldsymbol{q}_{\text {teq }}(\mathbf{p s i})\end{array}$ & $\begin{array}{c}\text { Point 23 } \\
\boldsymbol{q}_{\text {teq }} \text { (psi) }\end{array}$ & $\begin{array}{c}\text { Point 28 } \\
\boldsymbol{q}_{\text {teq }}(\mathbf{p s i )}\end{array}$ & $\begin{array}{c}\text { Point 30 } \\
\boldsymbol{q}_{\text {teq }}(\mathbf{p s i})\end{array}$ & COV $\boldsymbol{q}_{\text {teq }}$ \\
\hline 2.5 & 266 & 148 & 150 & 265 & 136 & 0.39 \\
\hline 5.0 & 249 & 161 & 168 & 239 & 148 & 0.25 \\
\hline 7.5 & 247 & 168 & 201 & 218 & 172 & 0.16 \\
\hline 10.0 & 259 & 204 & 228 & 218 & 244 & 0.09 \\
\hline 12.5 & 274 & 252 & 267 & 232 & 360 & 0.18 \\
\hline 15.0 & 299 & 313 & 333 & 240 & 516 & 0.31 \\
\hline 17.5 & 340 & 385 & 409 & 261 & 642 & 0.35 \\
\hline 20.0 & 392 & 446 & 466 & 303 & 722 & 0.34 \\
\hline 22.5 & 455 & 491 & 516 & 355 & 787 & 0.31 \\
\hline 25.0 & 536 & 533 & 583 & 414 & 844 & 0.24 \\
\hline 27.5 & 623 & 588 & 663 & 481 & 906 & 0.21 \\
\hline 30.0 & 710 & 660 & 737 & 548 & 973 & 0.18 \\
\hline 32.5 & 799 & 733 & 811 & 614 & 1036 & 0.16 \\
\hline 35.0 & 894 & 802 & 888 & 674 & 1089 & 0.13 \\
\hline 37.5 & 1002 & 866 & 972 & 736 & 1137 & 0.11 \\
\hline Avg & -- & -- & -- & -- & -- & $\mathbf{0 . 2 3}$ \\
\hline
\end{tabular}




\section{Results}

\subsection{Overview}

This chapter provides the results of all CPT and SASW tests as well as the results of all variability and additional analyses. Section 5.2 contains plots of $q_{t}$ vs $z$ for all CPT soundings as well as an initial variability analysis. Detailed CPT results for all points including all associated measurements and correlations can be found in the 2011 DSI report. Section 5.3 provides the results of SASW testing in the form of phase velocity dispersion curves for all points and all groups. Variability correlations between dispersion curves and CPT data utilizing the preliminary method are provided in Section 5.4. Finally, variability correlations, general results, and additional correlations utilizing the EWM are presented in Section 5.5.

\subsection{CPT Measurements and Initial Comparison}

After establishing the UWL as a viable site and obtaining CPT measurements from Reitz \& Jens, Inc., a preliminary examination of the raw and processed data was conducted. Processed data were selectively verified from raw data and found to be accurate in all cases. For each point, processed CPT data incudes $q_{t}, f_{s}, u_{m}$, and several other correlated values given at 2.5 -ft intervals starting at a depth of $1.25 \mathrm{ft}$ and extending to a depth of $36.25 \mathrm{ft}$ or cone refusal. For each depth, given measurements are not point measurements, but averages across the 2.5 -ft interval. For example, measurements $\left(q_{t}, f_{s}, u_{m}\right)$ provided for a depth of $1.25 \mathrm{ft}$ are average measurements across the interval from 0 to $2.5 \mathrm{ft}$ just as measurements provided for $21.25 \mathrm{ft}$ are average measurements across the interval from 20 
to $22.5 \mathrm{ft}$. Plots of $q_{t}$ vs $z$ for all points are included in Appendix A. Additionally, CPT measurements and all associated plots can be found in the 2011 DSI report.

Generally, CPT soundings across the UWL show low $q_{t}(0-300 \mathrm{psi})$ for depths from 0 to $15 \mathrm{ft}$ and then progressively increase to around 3000 psi at a depth of $35 \mathrm{ft}$. Plots of $q_{t}$ vs $z$ for Group 23, the northwesternmost group, are provided in Figure 5.1. Most points initially show $q_{t}$ of around 300 psi or less for the first 10 to $15 \mathrm{ft}$. After reaching 10 to 15 $\mathrm{ft}$ in depth, stiffness gradually increases to around 2600 psi at $35 \mathrm{ft}$ with Points 16 and 23 having $q_{t}$ as great as 4400 psi. One exception to the general trend is Point 30 which becomes stiff at around $10 \mathrm{ft}$, then rapidly drops to around 700 psi (Appendix A, Figure A.18). Finally of all $q_{t}$ vs $z$ plots in Group 23, the most scatter is observed in Points 18 and 30 (Appendix A, Figures A.6 \& A.18) with $q_{t}$ varying in excess of 1500 psi over only $2.5 \mathrm{ft}$ in each case.

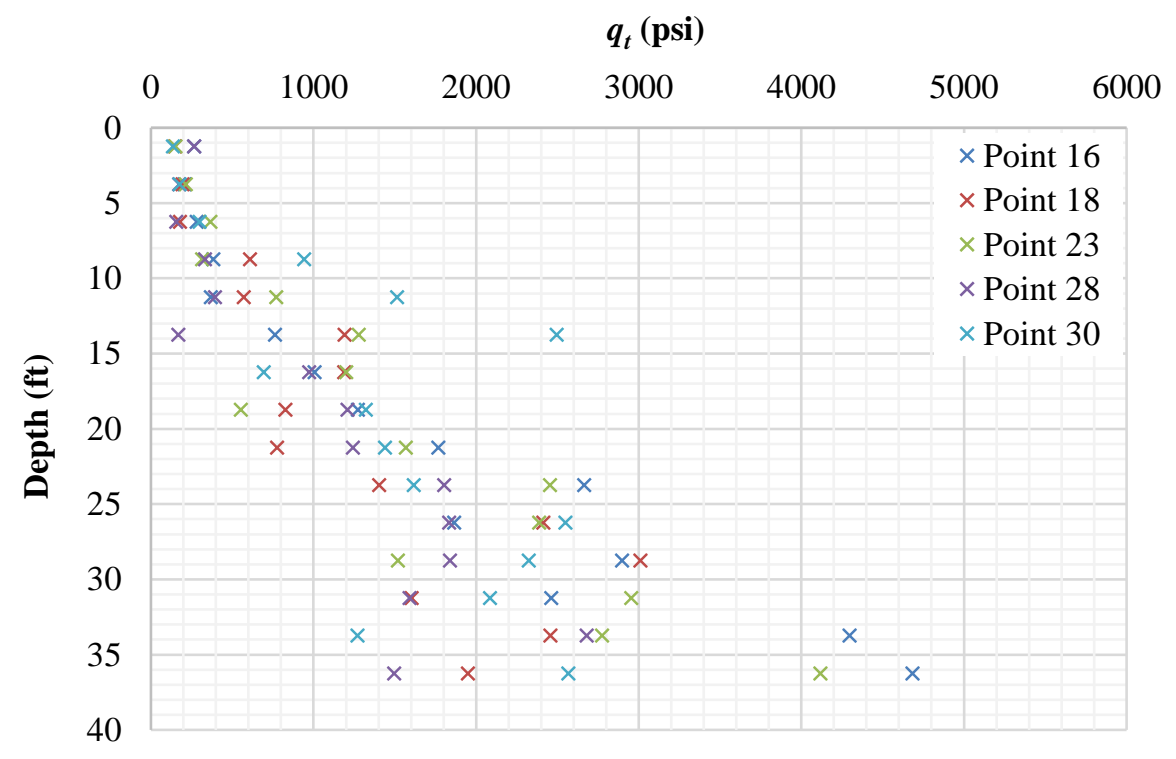

Figure 5.1 - Group 23 tip resistance $\left(q_{t}\right)$ vs depth $(z)$. 


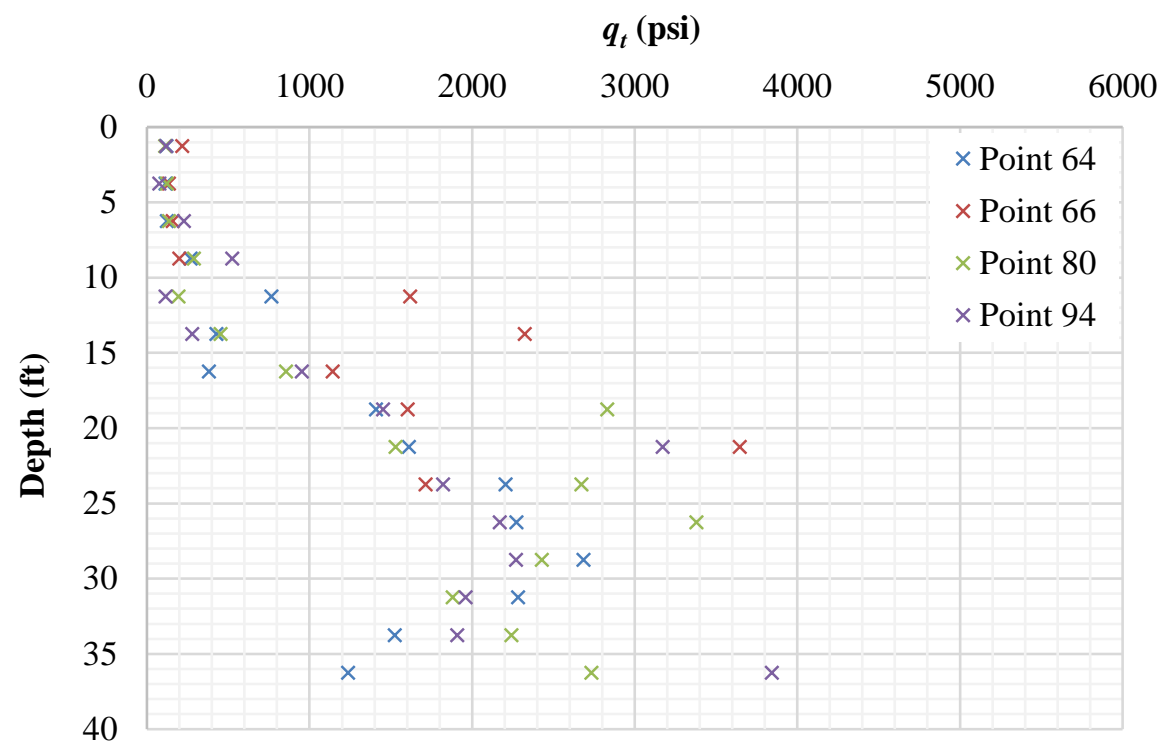

Figure 5.2 - Group 80 tip resistance $\left(q_{t}\right)$ vs depth $(z)$.

CPT soundings for the northwesternmost group south of Labadie Bottom Rd., Group 80 , are also quite soft in the upper 12 to $15 \mathrm{ft}$ with most $q_{t}$ measurements less than $400 \mathrm{psi}$ (Figure 5.2). Corrected tip resistance then progressively increases to approximately 2400 psi at $35 \mathrm{ft}$. Two of the four points in Group 80 show significant scatter with more than 1500 psi variation in $q_{t}$ across less than $2.5 \mathrm{ft}$ in depth. The most atypical sounding in Group 80 is Point 64 (Appendix A, Figure A.22), which shows a typical increase in $q_{t}$ until about $30 \mathrm{ft}$ at which point $q_{t}$ gradually decreases to about $1200 \mathrm{psi}$ at $35 \mathrm{ft}$ of depth. 


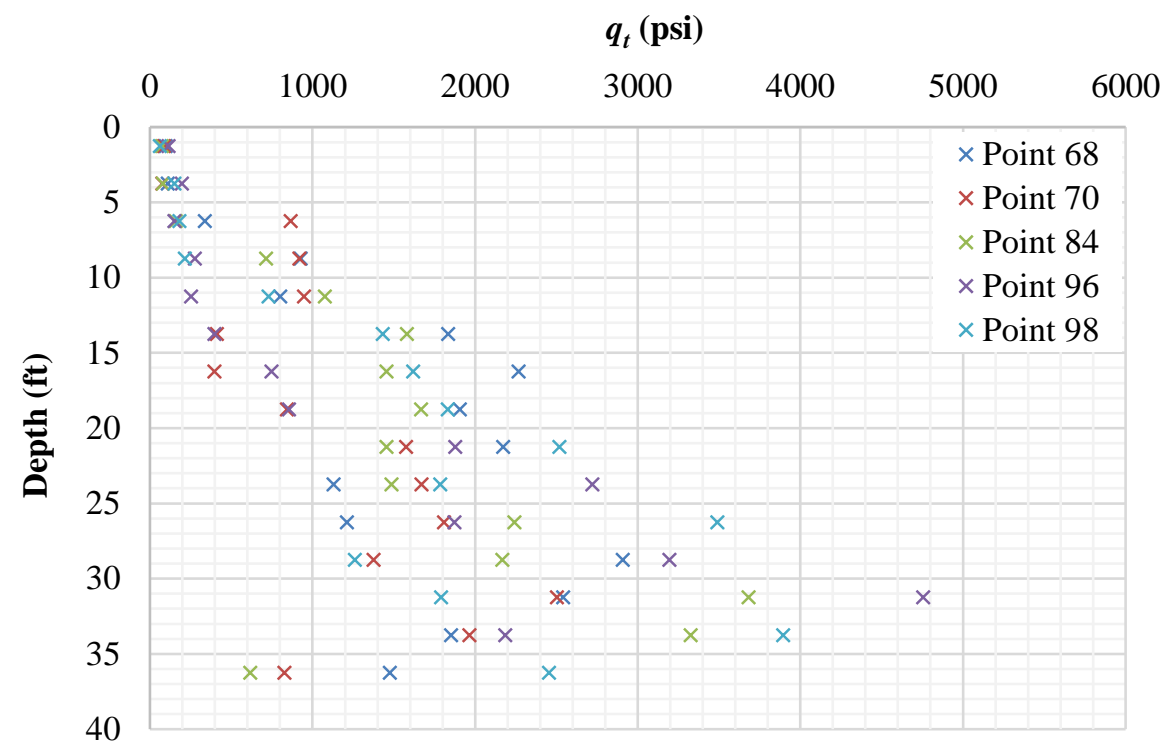

Figure 5.3 - Group 84 tip resistance $\left(q_{t}\right)$ vs depth $(z)$.

Group 84 is the northeasternmost group south of Labadie Bottom Rd. For this group, $q_{t} \mathrm{Vs} z$ displays significant scatter with four of the five points having variation in $q_{t}$ greater than 1500 psi over less than $2.5 \mathrm{ft}$ of depth (Figure 5.3). Atypical soundings include Points 70 and 84 which both have soft material with $q_{t}$ of approximately 700 psi at $35 \mathrm{ft}$ (Appendix A, Figure A.38 \& A.39). Also, Points 84 and 96 have stiff layers with $q_{t}$ in excess of 3800 psi at a depth of approximately $32 \mathrm{ft}$ (Appendix A, Figures A.39 \& A.40). Finally, the most atypical sounding is Point 98 where variation in excess of 2000 psi is repeatedly encountered from 20 to $35 \mathrm{ft}$ (Appendix A, Figure A.41). 


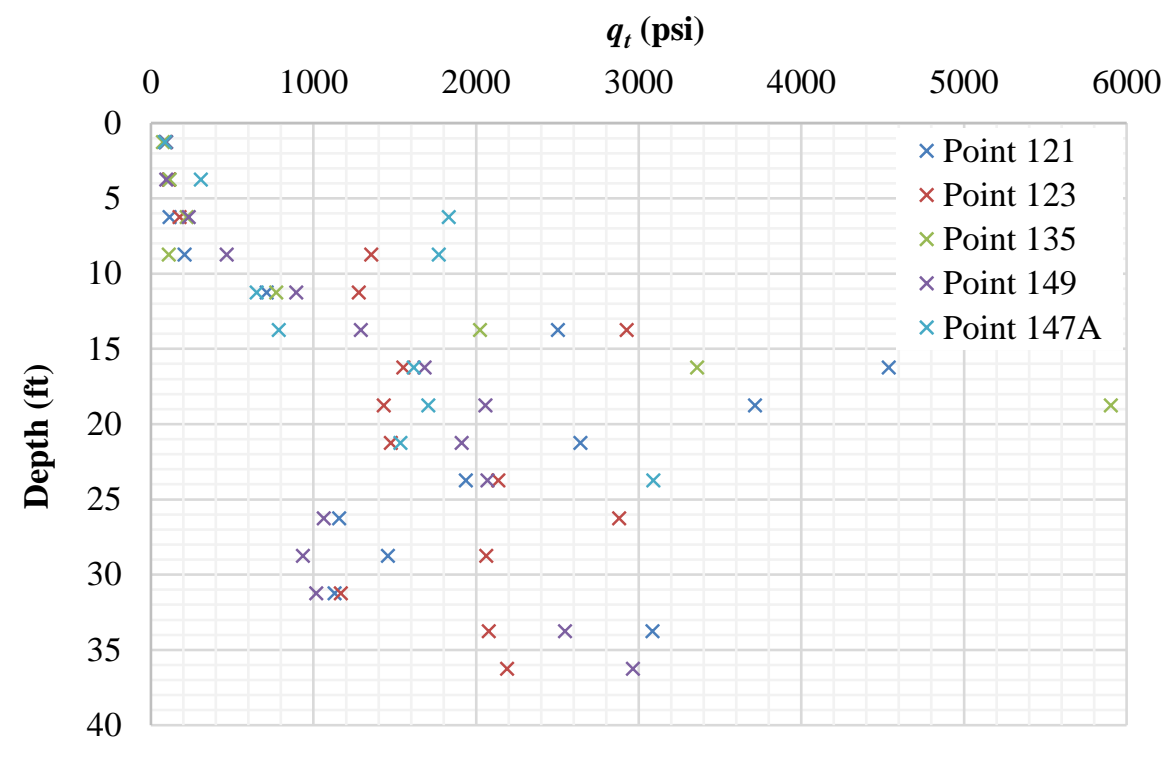

Figure 5.4 - Group 135 tip resistance $\left(q_{t}\right)$ vs depth $(\mathrm{z})$.

Group 135, the center group south of Labadie Bottom Rd., shows the most variability of all group $q_{t}$ vs $z$ plots (Figure 5.4). Two of the five soundings were refused at less than $35 \mathrm{ft}$ with Point 135 having refusal at $16 \mathrm{ft}$ and Point 147A having refusal at $25 \mathrm{ft}$ (Appendix A, Figures A.51 \& A55). Point 147A is also unusually stiff from 6 to $9 \mathrm{ft}$ with $q_{t}$ of around 1800 psi. Point 121 shows $q_{t}$ rapidly increase from 200 psi at $9 \mathrm{ft}$ to 4500 psi at $15 \mathrm{ft}$ followed by a decline to only $1200 \mathrm{psi}$ at $30 \mathrm{ft}$ (Appendix A, Figure A.43). Point 149 also exhibits unusual behavior with a soft section (1000 psi) from about 25 to $32 \mathrm{ft}$ (Appendix A, Figure A.47). Of the five soundings in Group 135, four show changes in $q_{t}$ in excess of 1500 psi over approximately $2.5 \mathrm{ft}$ in depth. 


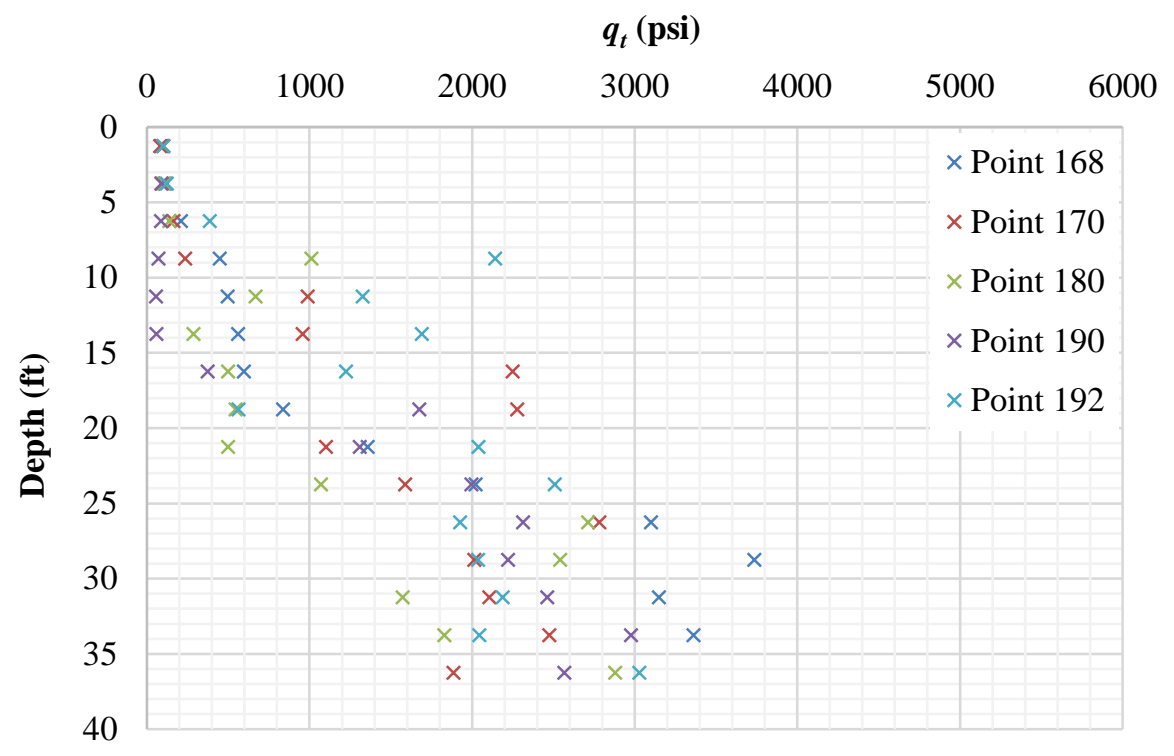

Figure 5.5 - Group 180 tip resistance $\left(q_{t}\right)$ vs depth $(z)$.

The southwesternmost group, Group 180, shows more consistency in the general trend of $q_{t}$ vs $z$ while individual soundings display some scatter (Figure 5.5). The most atypical sounding is Point 190 where $q_{t}$ is less than 100 psi from the surface to $15 \mathrm{ft}$, then sharply increases to 1700 psi at $20 \mathrm{ft}$ (Appendix A, Figure A.75). Of the five soundings in Group 180, two have changes in $q_{t}$ greater than 1500 psi across less than $2.5 \mathrm{ft}$ of depth.

Finally, the southeasternmost group, Group 184, is typical until about $25 \mathrm{ft}$ at which point soundings show more variability (Figure 5.6). Of the five soundings in Group 180, three have greater than 1500 psi variability in $q_{t}$ across less than $2.5 \mathrm{ft}$ of depth. Point 184 has an extremely stiff inclusion at $25 \mathrm{ft}$ with $q_{t}$ near 5500 psi followed by below average stiffness of only about 1500 psi at $35 \mathrm{ft}$ (Appendix A, Figure A.91). Point 196 is also atypical with a sharp decrease in $q_{t}$ from 2300 psi at $29 \mathrm{ft}$ to only 400 psi at $34 \mathrm{ft}$ (Appendix A, Figure A.99). 


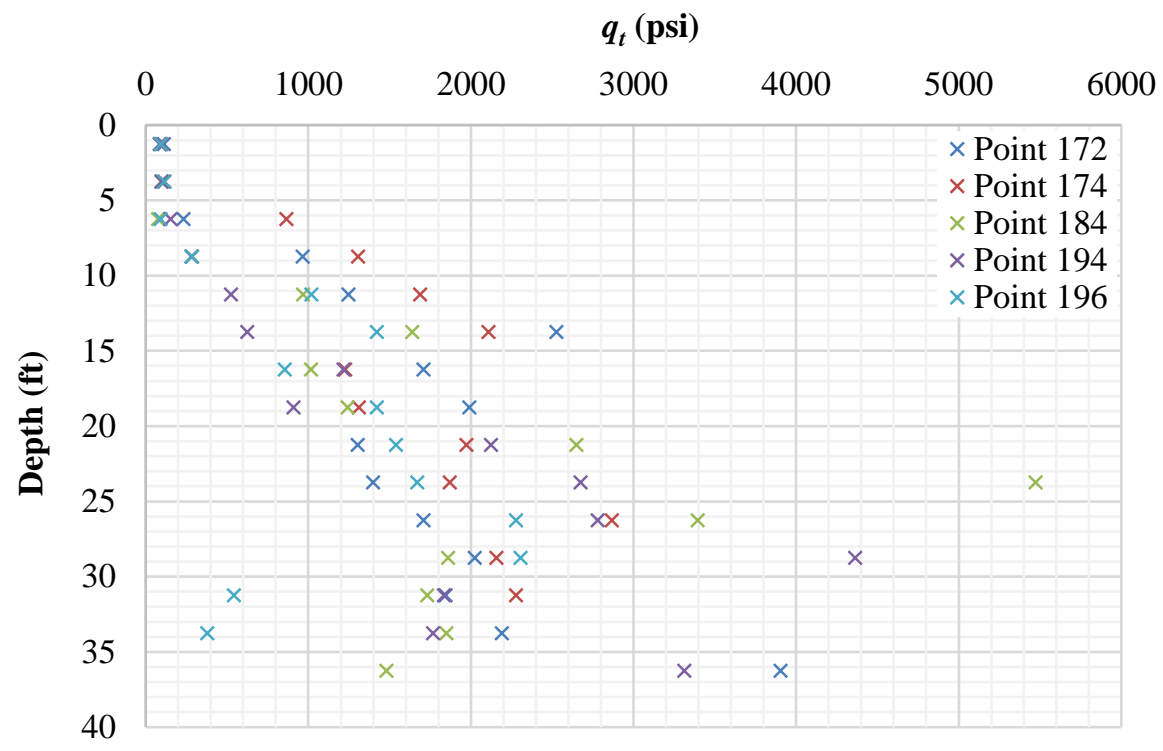

Figure 5.6 - Group 184 tip resistance $\left(q_{t}\right)$ vs depth $(z)$.

\subsubsection{Verification of Selected CPT Soundings}

The 2011 DSI report includes several CPT soundings taken at approximately the same physical locations (within $15 \mathrm{ft}$ ). In many cases, the additional test was conducted to verify shallow tip refusal, but there are also cases where additional tests were performed to verify the precision of the measurements. Figures 5.7, 5.8, and 5.9 show side-by-side CPT measurements. The average difference in CPT measurements taken at the same depth for Points 66, 135, and 168 is approximately $30 \%$. This $30 \%$ difference, however, is largely due to differences in $q_{t}$ at depths greater than $25 \mathrm{ft}$ for Point 168 . When those specific measurements are not considered, the average difference is reduced to approximately $18 \%$. For all nine locations where a redundant CPT sounding was taken, the mean difference in $q_{t}$ is approximately $22 \%$. 


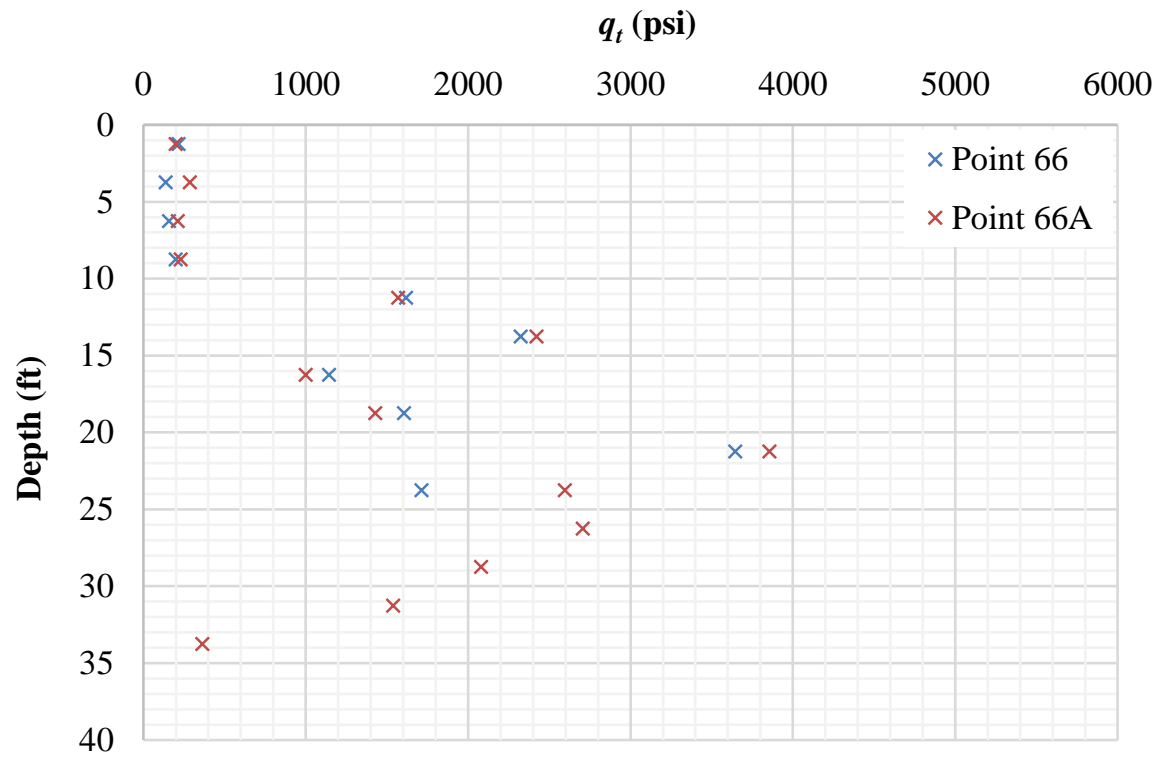

Figure 5.7 - Point 66 and 66A tip resistance $\left(q_{t}\right)$ vs depth $(z)$.

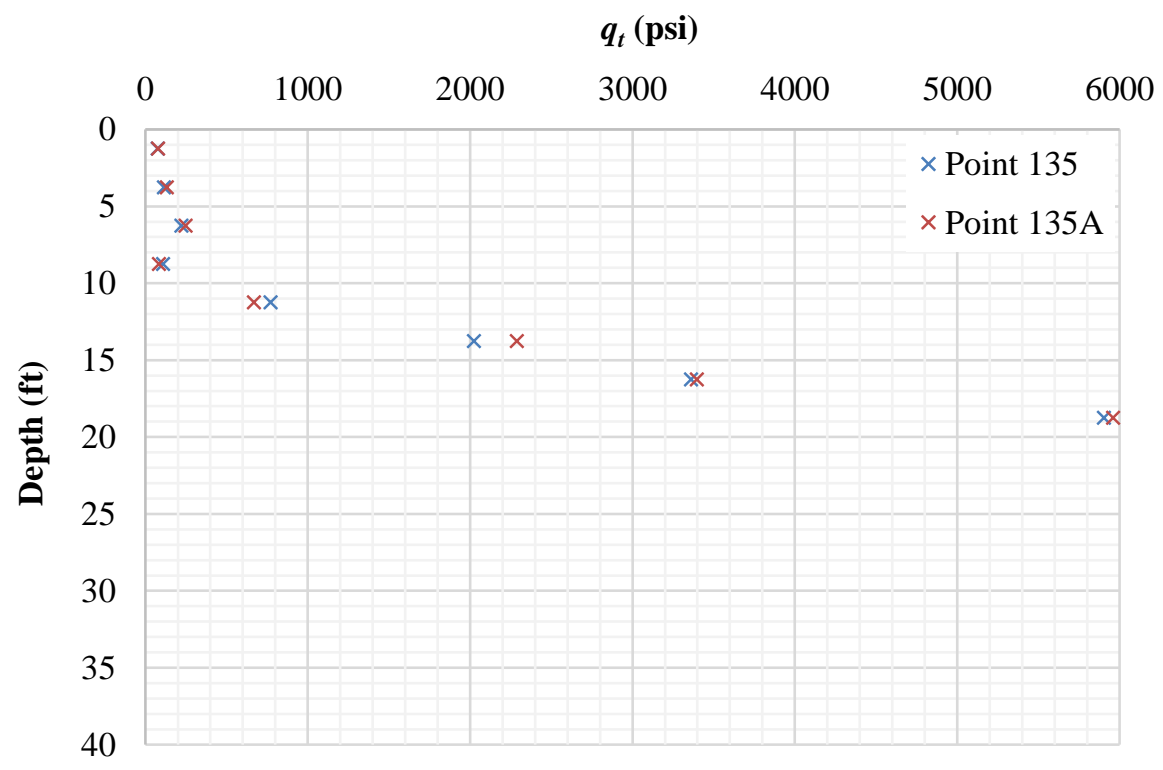

Figure 5.8 - Point 135 and 135A tip resistance $\left(q_{t}\right)$ vs depth $(\mathrm{z})$. 


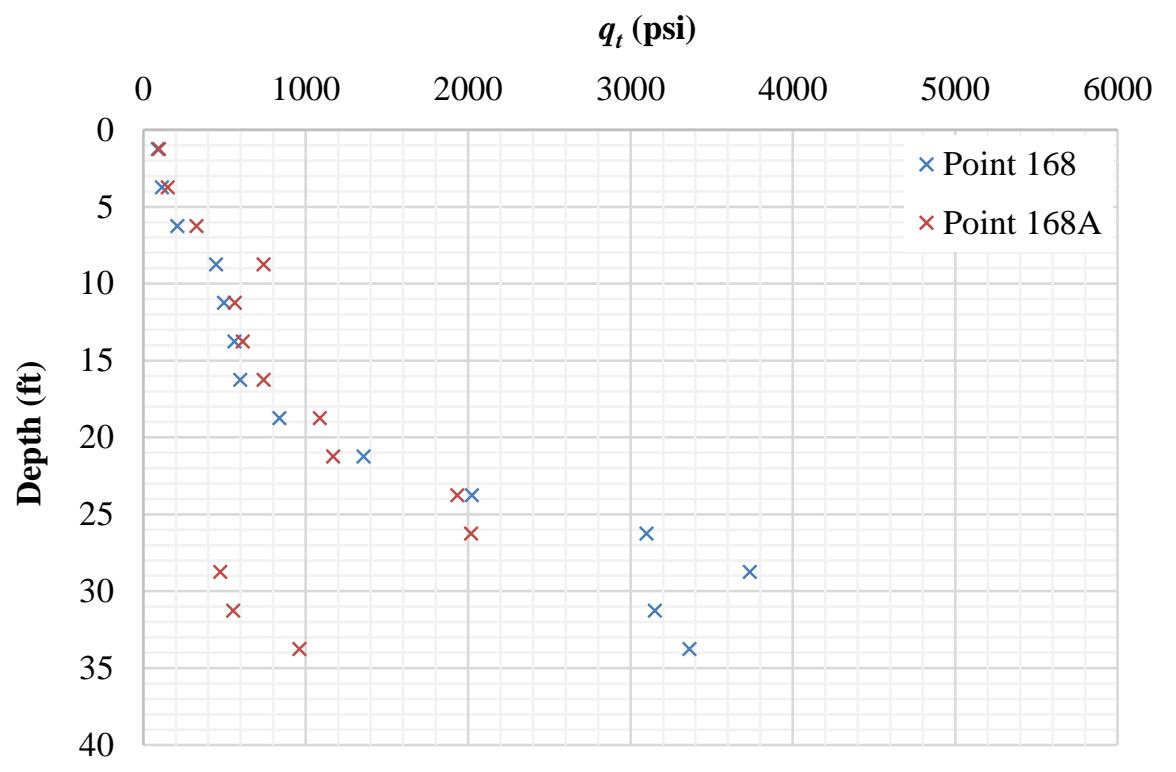

Figure 5.9 - Point 168 and 168A tip resistance $\left(q_{t}\right)$ vs depth $(z)$.

\subsubsection{Preliminary Variability Analysis of CPT Data}

Prior to any SASW testing, a brief variability analysis of CPT data was conducted to examine the suitability of the UWL site and the preliminary method. Specifically, the analysis was intended to determine whether a sufficient range in variability existed amongst groups of CPT measurements. This variability analysis was carried out as described in Section 4.5.1 and considered variability in $q_{t}$ and $f_{s}$. Figure 5.10 shows that among the six groups analyzed, the average $\mathrm{COV}$ in $q_{t}$ for each group ranges from about 0.35 to 0.56 . Similarly, the average $\mathrm{COV}$ in $f_{s}$ ranges from 0.35 to 0.54 . It also shows that utilizing this method, variability in $q_{t}$ is related to variability in $f_{s}$ at an approximate $1: 1$ ratio. Based on these results, the range in variability at the UWL site was deemed sufficient to proceed with SASW testing. Further, the approximate 1:1 correlation between mean COVs for $q_{t}$ and $f_{s}$ served as an initial validation of the preliminary method. 


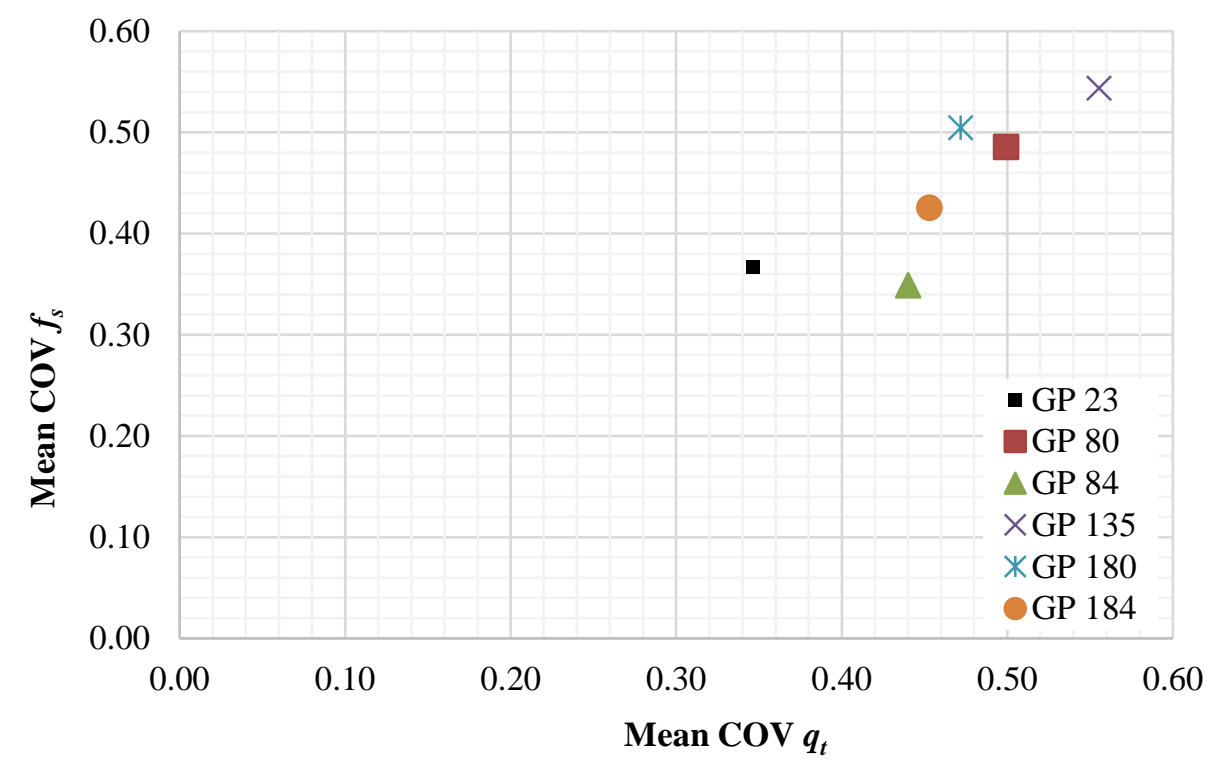

Figure 5.10 - Preliminary variability analysis of UWL CPT data.

\subsection{SASW Dispersion Curves}

Phase velocity dispersion curves were obtained from SASW testing as described in Section 4.3 and are provided for each point in Appendix A. Generally, the UWL site yields $V_{p h}$ between 150 and $350 \mathrm{ft} / \mathrm{s}$ for the smallest $\lambda(1-5 \mathrm{ft}$ ) and gradually increases to 490 to $730 \mathrm{ft} / \mathrm{s}$ for the largest wavelengths $(50-75 \mathrm{ft})$. When calculated utilizing the preliminary method, however, dispersion curves show much less variability than CPT data with mean COVs ranging from approximately 0.07 to 0.1 . 


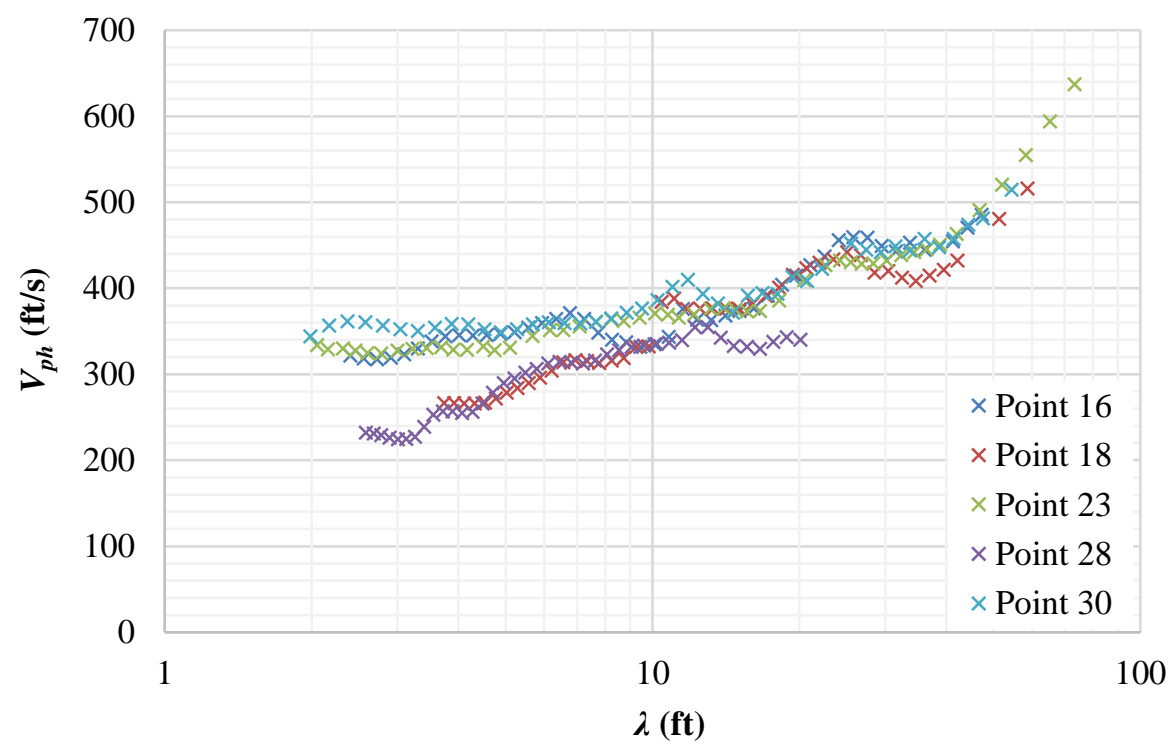

Figure 5.11 - Group 23 dispersion curves.

The northwesternmost group, Group 23, has two distinct trends in dispersion curves for $\lambda$ less than $20 \mathrm{ft}$ (Figure 5.11). The first trend is exhibited in Points 16, 23, and 30 with $V_{p h}$ starting at around $350 \mathrm{ft} / \mathrm{s}$ and increasing at a rate of about $4 \mathrm{ft} / \mathrm{s} / \mathrm{ft}$ to around $450 \mathrm{ft} / \mathrm{s}$ at $\lambda$ of $25 \mathrm{ft}$. From $\lambda$ of 25 to $35 \mathrm{ft}, V_{p h}$ decreases slightly and at $\lambda$ greater than $35 \mathrm{ft}, V_{p h}$ increases at a rate of about $4.3 \mathrm{ft} / \mathrm{s} / \mathrm{ft}$. The second trend is seen in Points 18 and 28 which have $V_{p h}$ that are 15 to $25 \%$ lower than the rest of the group. However, for Point $28, V_{p h}$, for $\lambda$ longer than approximately $10 \mathrm{ft}$ is not available due to poor data quality. The COV in $V_{p h}$ for Group 23 as calculated utilizing the preliminary method is 0.072 . 


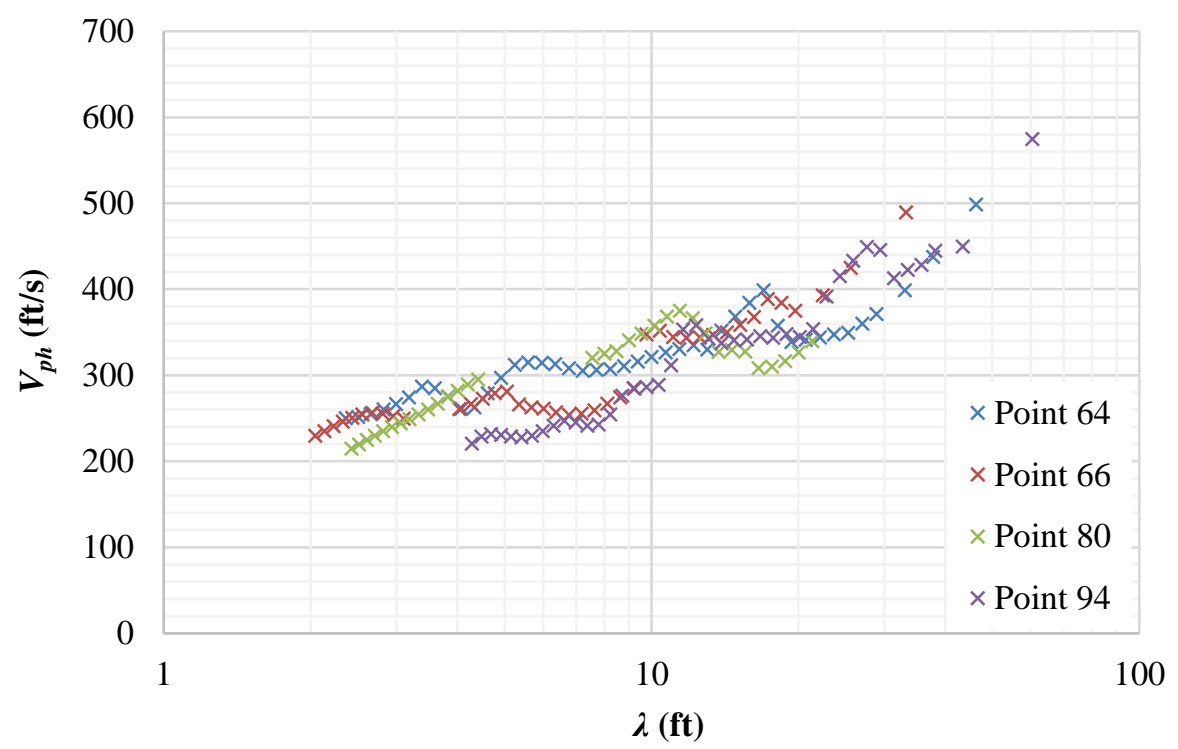

Figure 5.12 - Group 80 dispersion curves.

Group 80, the northwesternmost group south of Labadie Bottom Rd., shows an increase in $V_{p h}$ from about $250 \mathrm{ft} / \mathrm{s}$ at small $\lambda$ to around $375 \mathrm{ft} / \mathrm{s}$ at $\lambda$ of $15 \mathrm{ft}$ (Figure 5.12). From $\lambda$ of 15 to $25 \mathrm{ft}, V_{p h}$ remains fairly constant but increase at a rate of approximately 4 $\mathrm{ft} / \mathrm{s} / \mathrm{ft}$ for longer wavelengths. Point 94 is notably different from the rest of the group in that $V_{p h}$ is around $50 \mathrm{ft} / \mathrm{s}$ slower from $\lambda$ of 4 to $8 \mathrm{ft}$. Utilizing the preliminary method, the $\mathrm{COV}$ of $V_{p h}$ for Group 80 is 0.085 . 


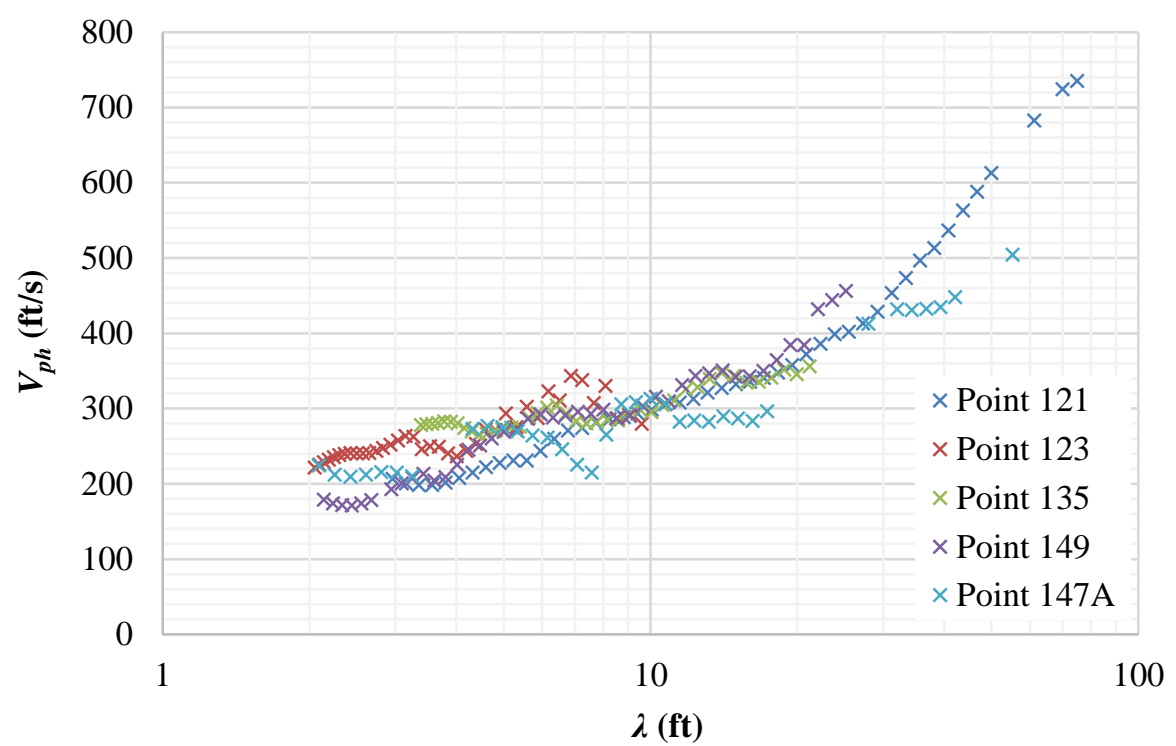

Figure 5.13 - Group 135 dispersion curves.

Dispersion curves in Group 135, the center group south of Labadie Bottom Rd., have $V_{p h}$ of around $200 \mathrm{ft} / \mathrm{s}$ at small $\lambda$ and increase to around $350 \mathrm{ft} / \mathrm{s}$ over the first $15 \mathrm{ft}$ of $\lambda$ (Figure 5.13). Of the five SASW tests in Group 135, only two (121 \& 147A) yielded results beyond $\lambda$ of $30 \mathrm{ft}$. At these wavelengths, Point 147A has $V_{p h}$ approximately $10 \%$ lower than Point 121. Point 121 also yields the highest $V_{p h}$ of all UWL tests at $735 \mathrm{ft} / \mathrm{s}$ for $\lambda$ of $75 \mathrm{ft}$. Using the preliminary method, the COV of $V_{p h}$ for Group 135 is 0.079 . 


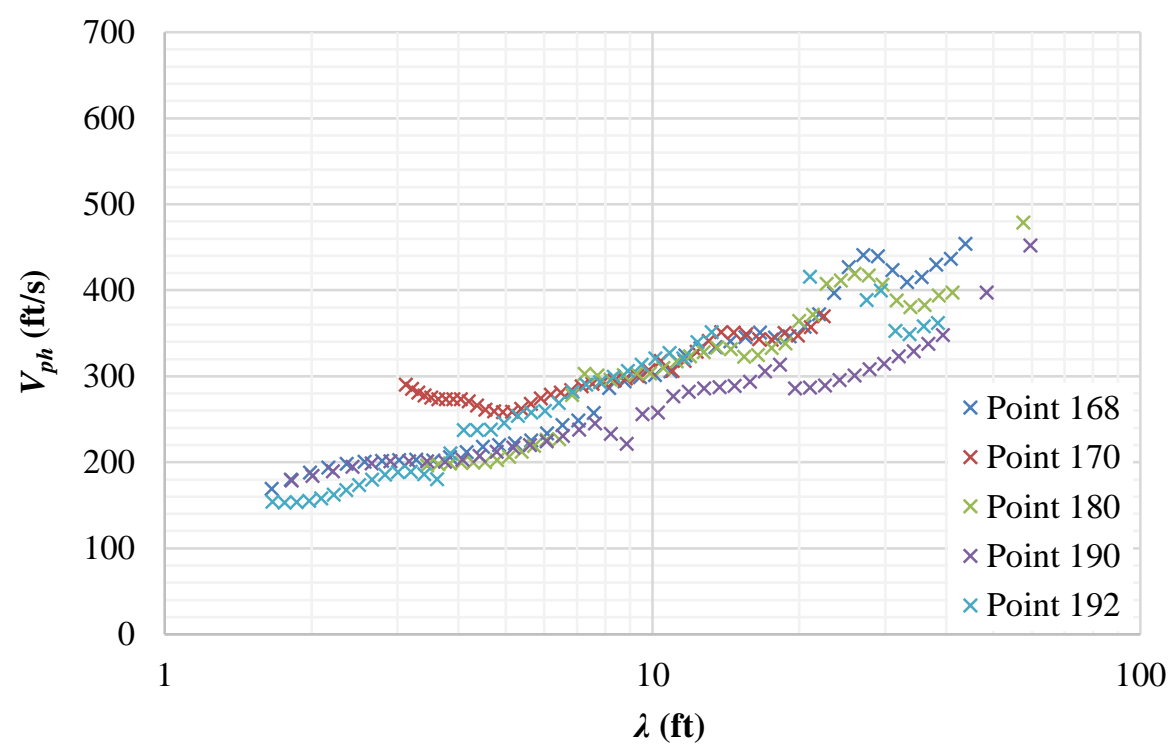

Figure 5.14 - Group 180 dispersion curves.

Group 180 is marked by significant variation in $V_{p h}$ for $\lambda$ less than $5 \mathrm{ft}$ and greater than $20 \mathrm{ft} . V_{p h}$ for $\lambda$ less than $5 \mathrm{ft}$ varies from $275 \mathrm{ft} / \mathrm{s}$ for Point 170 to $153 \mathrm{ft} / \mathrm{s}$ for Point 192 (Figure 5.14). $V_{p h}$ for all points except 190 then converge at $300 \mathrm{ft} / \mathrm{s}$ for $\lambda$ of $8 \mathrm{ft}$. At this $\lambda$, $V_{p h}$ for Point 190 is approximately $10 \%$ lower at $260 \mathrm{ft} / \mathrm{s}$. Beyond $\lambda$ of $20 \mathrm{ft}, V_{p h}$ varies by as much as $130 \mathrm{ft} / \mathrm{s}$ between points, but in all cases, increases at a rate of approximately $2.5 \mathrm{ft} / \mathrm{s} / \mathrm{ft}$. Group $180 V_{p h}$ has a COV of 0.099 as calculated with the preliminary method. 


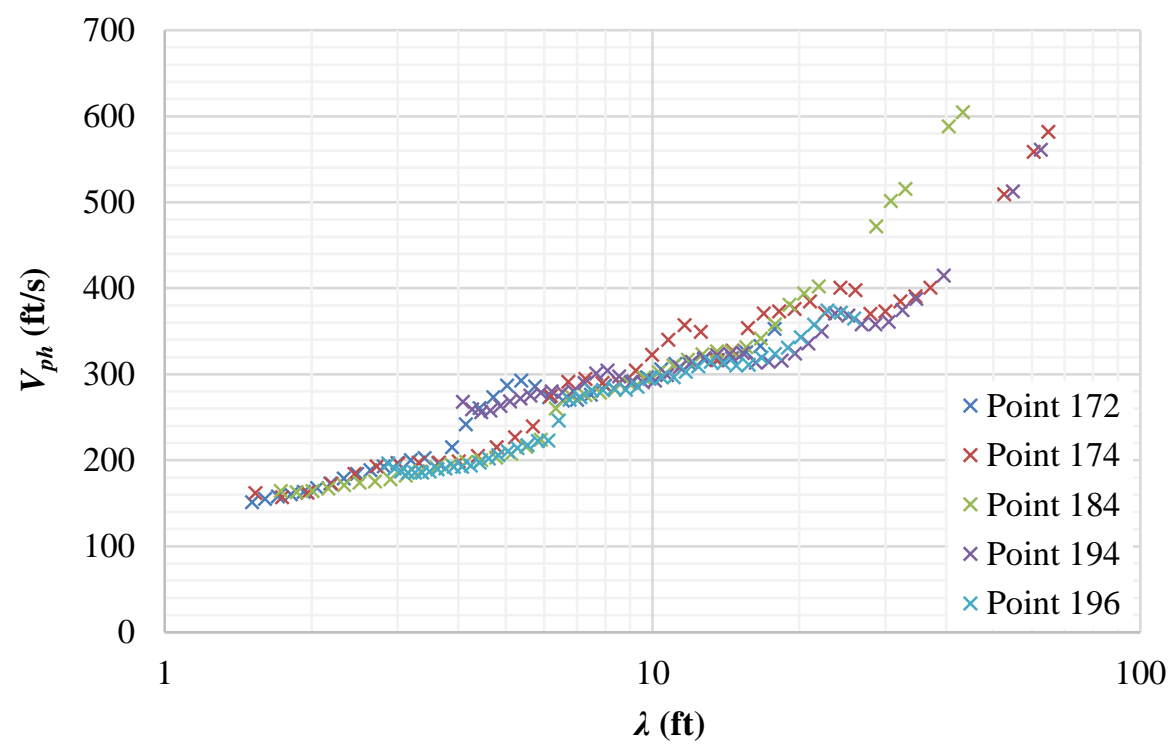

Figure 5.15 - Group 184 dispersion curves.

Finally, Group 184 dispersion curves present the least amount of variability with a $\mathrm{COV}$ of 0.071 . In all cases, $V_{p h}$ at small $\lambda$ is around $160 \mathrm{ft} / \mathrm{s}$ and increases at a rate of 12 $\mathrm{ft} / \mathrm{s} / \mathrm{ft}$ to around $200 \mathrm{ft} / \mathrm{s}$ at $\lambda$ of $4 \mathrm{ft}$ (Figure 5.15). All points then exhibit a marked increase in $V_{p h}$ to approximately $275 V_{p h}$ at $\lambda$ of $7 \mathrm{ft}$. $V_{p h}$ then increases at a rate of approximately 5 $\mathrm{ft} / \mathrm{s} / \mathrm{ft}$ to $313 \mathrm{ft} / \mathrm{s}$ at $\lambda$ of $15 \mathrm{ft}$. At $\lambda$ of $15 \mathrm{ft}$, Group 184 increases at a rate of $12 \mathrm{ft} / \mathrm{s} / \mathrm{ft}$ to around $375 \mathrm{ft} / \mathrm{s}$ at $\lambda$ of $20 \mathrm{ft}$ where $V_{p h}$ decreases slightly until $\lambda$ of $30 \mathrm{ft}$. Beyond $\lambda$ of $30 \mathrm{ft}$, Point 184 yields $V_{p h}$ about $40 \%$ higher than Points 194 and 174, however, $V_{p h}$ increases at approximately the same rate in all three cases.

\subsubsection{Verification of Selected SASW Tests}

For points where side-by-side CPT soundings were taken, side-by-side SASW tests were also conducted. Additional SASW tests were generally taken by moving the SASW array approximately 5 to $10 \mathrm{ft}$ perpendicular to the long axis of the array. Figures 5.16 to 
5.18 show the results of side-by-side SASW testing. The figures indicate that a close association exists in all cases with only a couple of exceptions. Figure 5.17 shows that for Point 135A, $V_{p h}$ varies significantly for $\lambda$ up to $6 \mathrm{ft}$. However, dispersion curves of Points 135 and $135 \mathrm{~A}$ are essentially the same for $\lambda$ greater than $6 \mathrm{ft}$. This deviation is most likely due to the stiffness of the upper two to three feet of topsoil. Also, Figure 5.18 shows that for Point 168, there is about a $50 \mathrm{ft} / \mathrm{s}$ difference in $V_{p h}$ for $\lambda$ of approximately $10 \mathrm{ft}$. This difference could be the result of variability in the subsurface or poor test data in the $10-\mathrm{ft}$ receiver spacing.

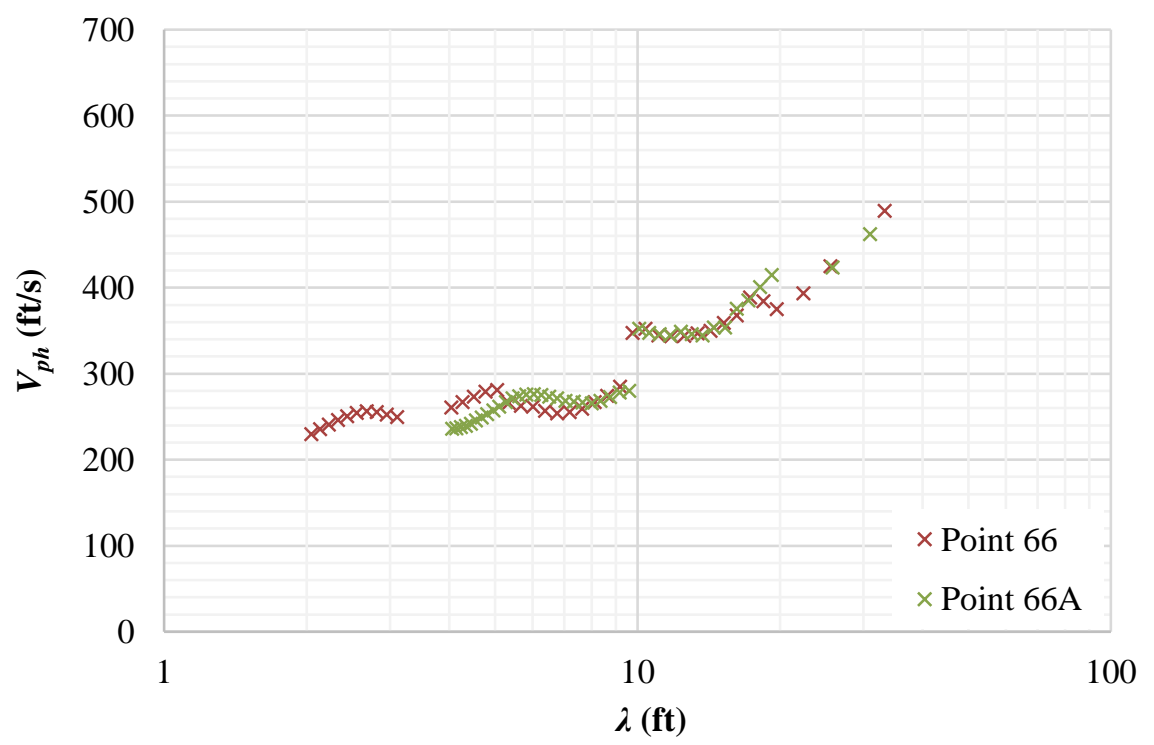

Figure 5.16 - Point 66 SASW verification. 


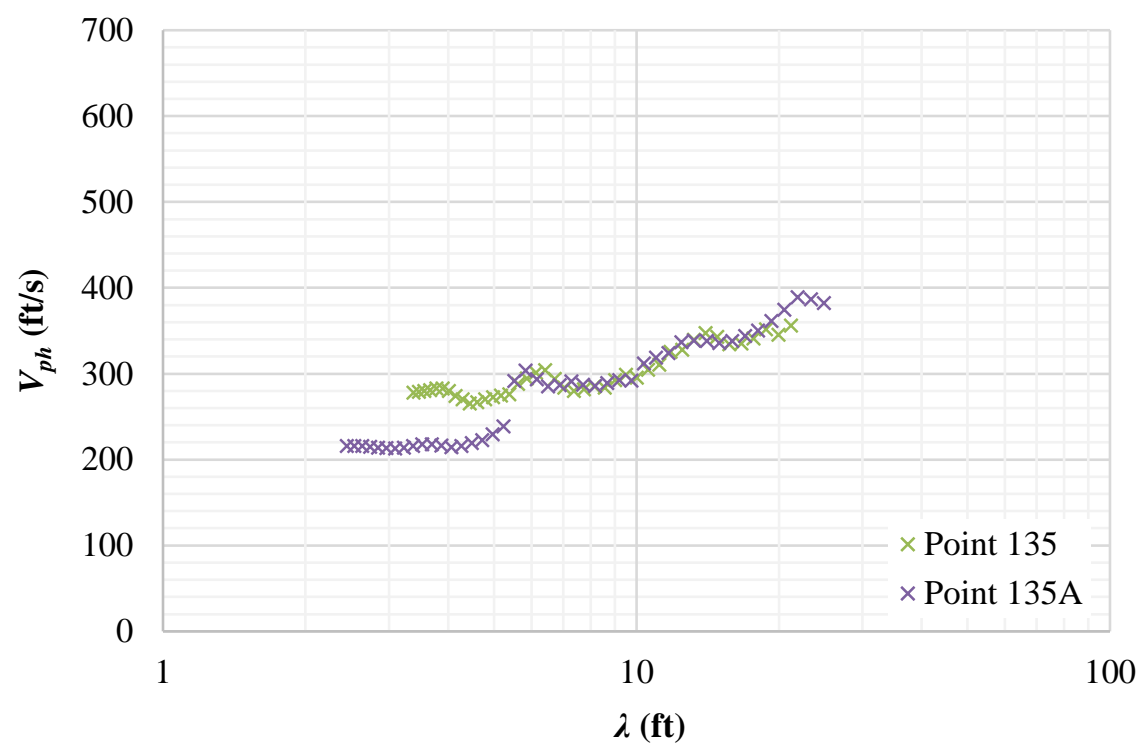

Figure 5.17 - Point 135 SASW verification.

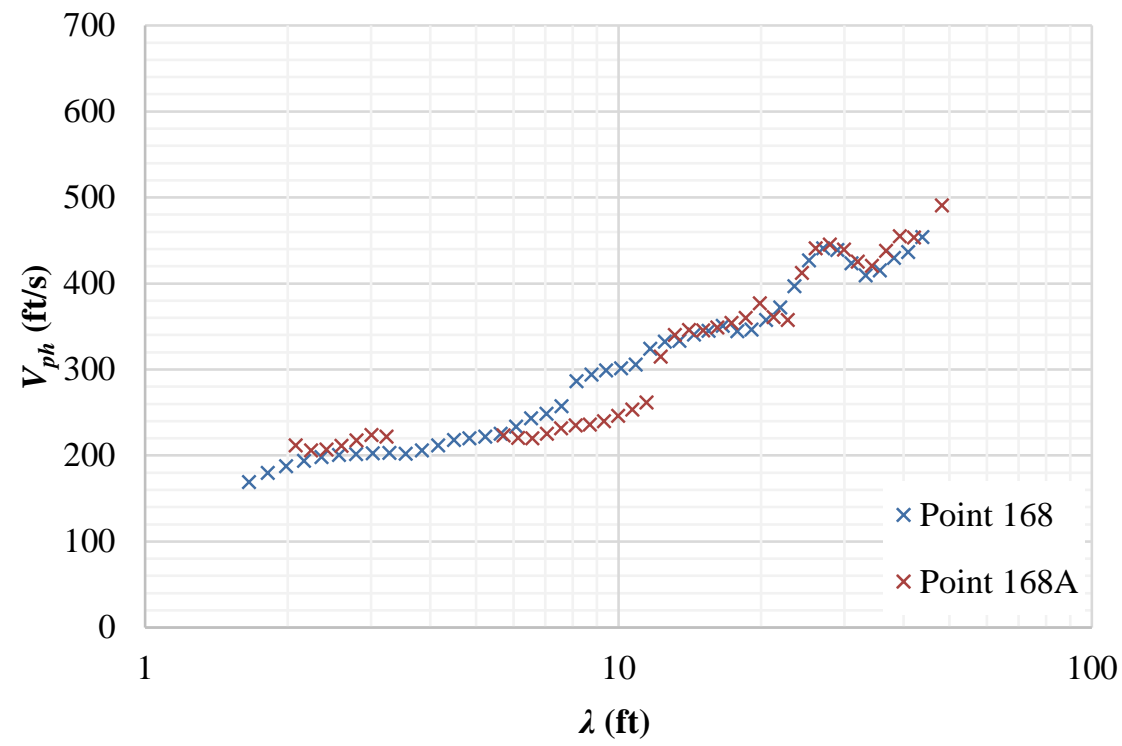

Figure 5.18 - Point 168 SASW verification. 


\subsection{Preliminary Variability Correlation}

After achieving COVs for CPT and SASW data for each group utilizing the preliminary method, COVs were plotted to determine whether a correlation exists and/or what improvements could be made. For this analysis, the COV of $q_{t}$ and the COV of $f_{s}$ for each group were plotted against the COV of $V_{p h}$ for the same. In both cases, following two observations are made:

1. The COV of CPT data is much larger than the COV of $V_{p h}$.

2. A statistically significant correlation does not exist.

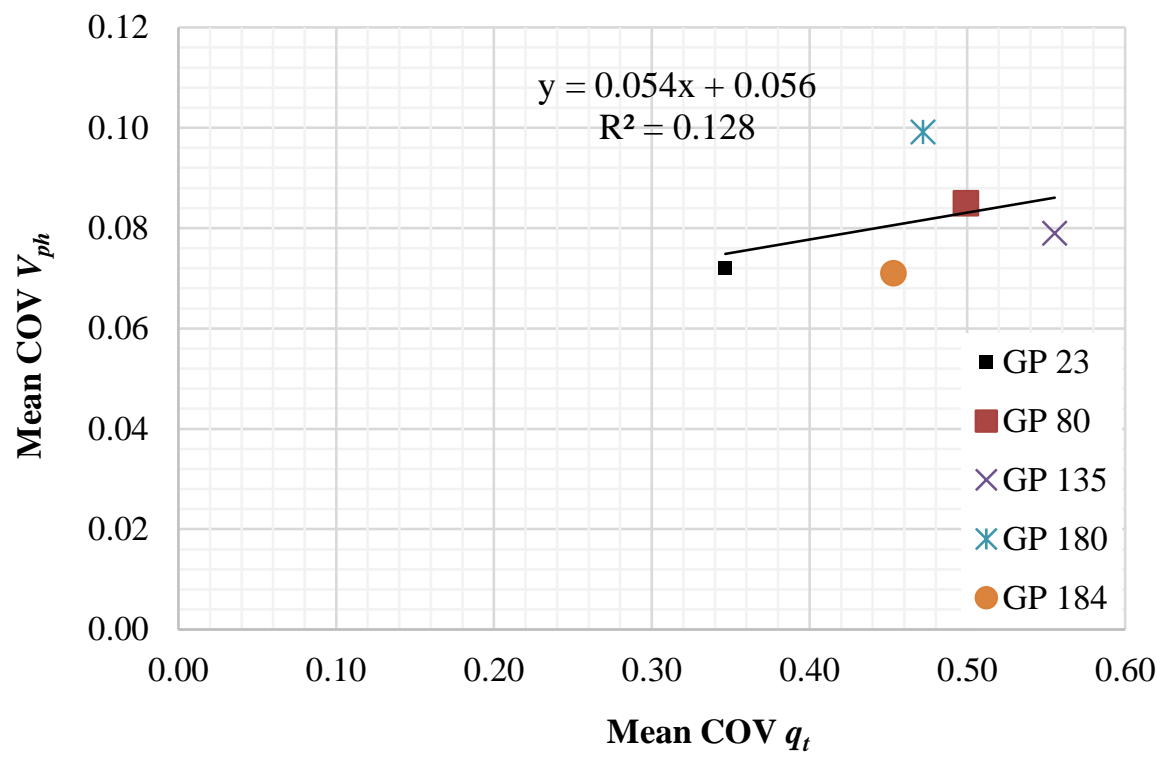

Figure 5.19 - Mean COV $V_{p h}$ vs Mean COV $q_{t}$.

A plot of the mean COV $q_{t}$ vs the mean COV $V_{p h}$ is shown in Figure 5.19. A regression analysis yields an $\mathrm{R}^{2}$ of 0.128 with a slope of 0.054 , an intercept of 0.056 , and a probability value (p-value) of 0.55 . The mean COV of $f_{s}$ vs the mean COV of $V_{p h}$ is shown in Figure 
5.20. In this case, the regression analysis is only slightly better with an $R^{2}$ of 0.36 , a slope of 0.099 , an intercept of 0.035 , and a p-value of 0.29 .

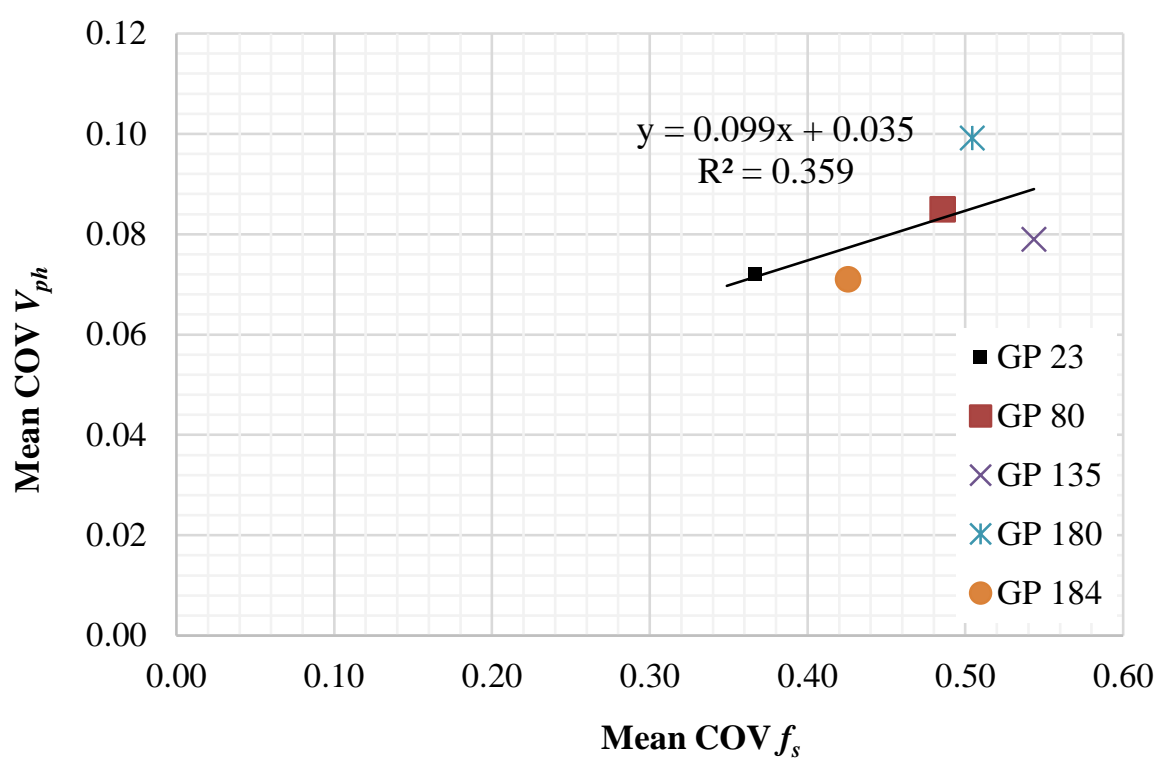

Figure 5.20 - Mean $\operatorname{COV} V_{p h}$ vs Mean $\operatorname{COV} f_{s}$.

\subsection{Equivalent Wavelength Method}

The methodology for the equivalent wavelength method (EWM) is described in detail in Section 4.5.2. The first step in application of the EWM is calculation of the equivalent corrected tip resistance $\left(q_{\text {teq }}\right)$ for desired $\lambda$. For this study, $q_{t e q}$ and $V_{p h}$ were calculated at each test point for wavelengths of 2.5 to $37.5 \mathrm{ft}$ on a 2.5 -ft interval. Individual plots of $q_{\text {teq }}$ vs $\lambda$ are available in Appendix A and group plots of $q_{\text {teq }}$ vs $\lambda$ are available in Appendix B.

Figures 5.21 and 5.22 are plots of $q_{t}$ vs $z$ and $q_{t e q}$ vs $\lambda$, respectively, for Point 30. Of note is the significant reduction in scatter in $q_{\text {teq }}$ vs $\lambda$ as well as the prominent increase in $q_{\text {teq }}$ at a wavelength of approximately $15 \mathrm{ft}$. Both of these phenomena are the result of weighted averaging of $q_{t}$ in accordance with Rayleigh wave energy distribution. In this 
case, a significant portion of energy for $\lambda$ greater than $15 \mathrm{ft}$ is propagated through the stiff soil layer encountered from 9 to $14 \mathrm{ft}$.

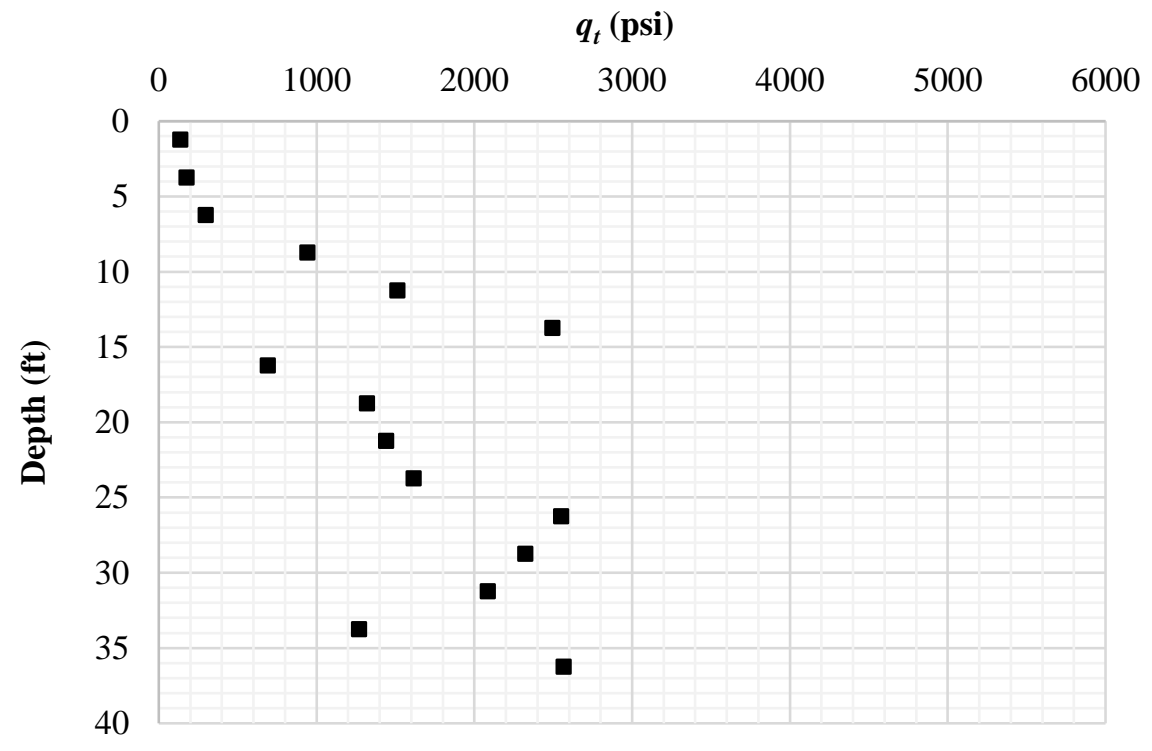

Figure 5.21 - Point $30 q_{t}$ vs $z$.

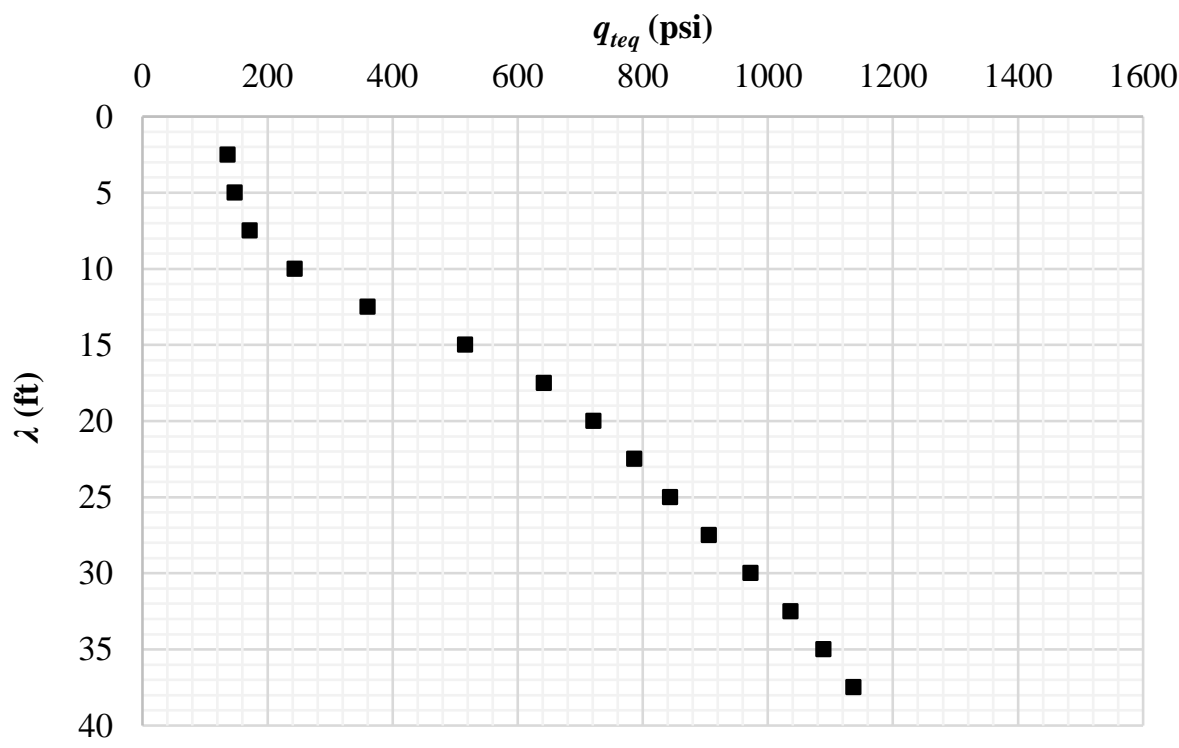

Figure 5.22 - Point $30 q_{t e q}$ vs $\lambda$. 
Applied to Group 23, the EWM transforms a scattered $q_{t}$ vs $z$ plot (Appendix B, Figure B.3) into a much more manageable $q_{\text {teq }}$ vs $\lambda$ plot as shown in Figure 5.23. The figure also shows, however, that for $\lambda$ less than $5 \mathrm{ft}$, there is significant variation in $q_{\text {teq }}$. This trend is also observed in Group 80 (Appendix B, Figure B.7) and is caused by significant variability in the stiffness of the upper $5 \mathrm{ft}$ of soil. At the UWL, variability in the upper $5 \mathrm{ft}$ is the result of several factors including time elapsed since last plowing, depth of plowing, root penetration, surface water and seepage conditions, and depth to the groundwater table. Beyond wavelengths of $10 \mathrm{ft}$, these factors are still incorporated in the calculation of $q_{\text {teq }}$, but their effect is less pronounced.

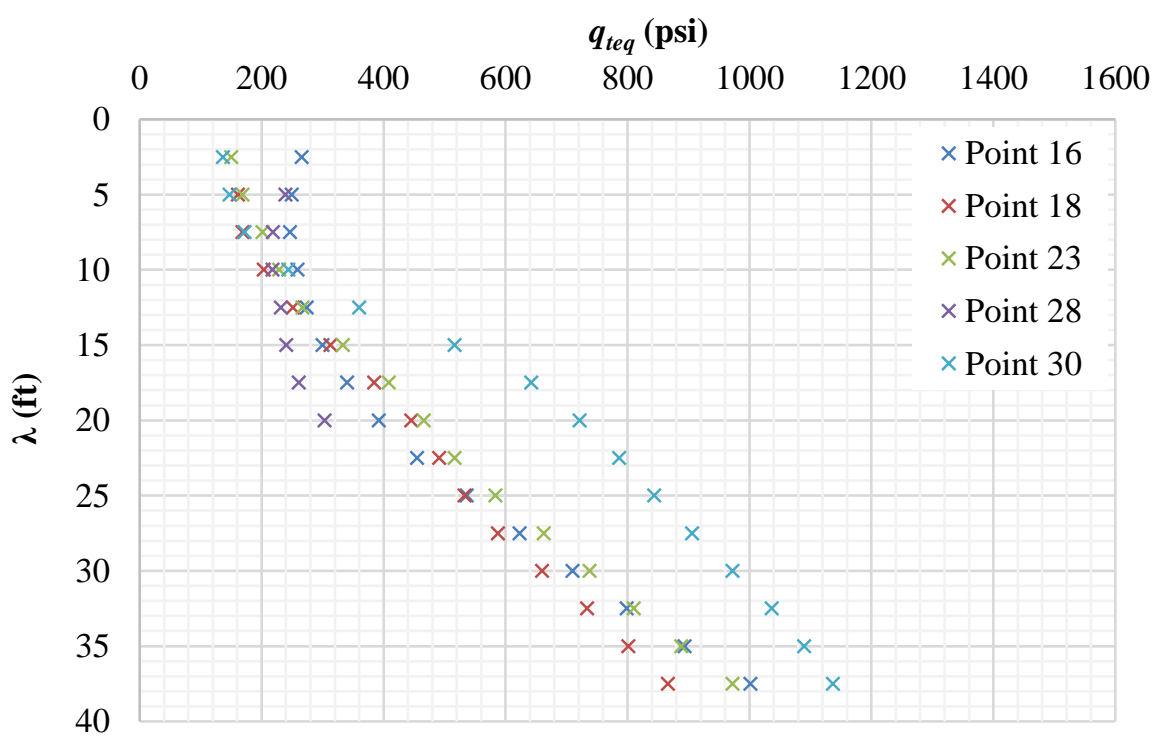

Figure 5.23 - Group 23 equivalent tip resistance $\left(q_{t e q}\right)$ vs wavelength $(\lambda)$.

Another trend observed in the Group $23 q_{t e q}$ vs $\lambda$ plot (Figure 5.23) is that significant spikes in $q_{t}$ at depths between 7 and $15 \mathrm{ft}$ result in a marked increase in $q_{t e q}$ for longer $\lambda$. This is especially noticeable for Points 30, 147A, and 192 of Groups 23, 135, and 180 
respectively (Appendix B). In each case, however, the increase in $q_{t e q}$ does not correspond to a noticeable increase in $V_{p h}$. This indicates that the CPT probe is passing through a relatively small, stiff inclusion or layer, that is not prevalent enough across the span of the site to cause an appreciable increase in $V_{p h}$.

\subsubsection{EWM Variability Correlation}

Utilizing the EWM, variability in $V_{p h}$ was compared to variability in $q_{\text {teq }}$ and $f_{\text {seq }}$. Figure 5.24 shows that in the case of $f_{\text {seq }}$, no discernable trend exists. The lowest mean $\mathrm{COV}$ in $f_{\text {seq }}$ is approximately 0.182 for Group 80 and the highest is 0.262 for Group 23. Groups 135 and 180 have similar COVs in $f_{\text {seq }}$ at 0.257 and 0.251 , respectively, and Group 184 has a COV of 0.208 . None of these values, however, are correlated to the mean COV of $V_{p h}$ in any meaningful way. Based on this finding and those of the preliminary method, no further evaluation of variability in $f_{s}$ or $f_{\text {seq }}$ relative to $V_{p h}$ will be presented.

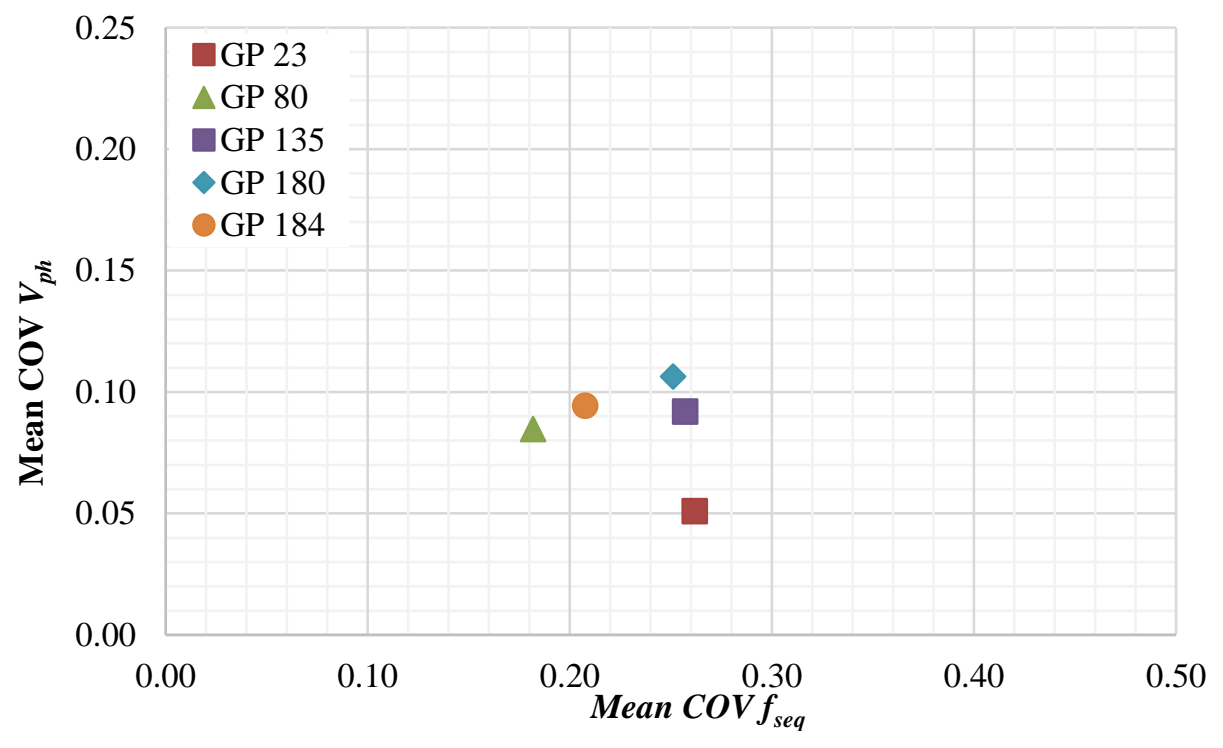

Figure 5.24 - Mean $\operatorname{COV} V_{p h}$ vs Mean $\operatorname{COV} f_{\text {seq. }}$ 
Unlike $f_{\text {seq }}$, variability in $q_{t e q}$ shows a much stronger correlation to variability in $V_{p h}$. Figure 5.25 shows the mean COV for $V_{p h}$ vs the mean COV of $q_{t e q}$ for each group. The lowest COVs for $q_{\text {teq }}$ and $V_{p h}$ are from Group 23 at 0.22 and 0.05 respectively. The highest mean COV for $q_{\text {teq }}$ is from Group 135 at 0.48 , however, this high COV is due almost exclusively to the high $q_{t e q}$ values of Point 147A (Appendix B, Figure B.12). The highest mean COV in $V_{p h}$ is from Group 180 where dispersion curves show consistent variation across the range of measured wavelengths. A regression analysis through the data presented in Figure 5.25 yields an $\mathrm{R}^{2}$ of 0.751 with a slope of 0.177 , an intercept of 0.019 , and a pvalue of 0.057 . In this case, a regression through the origin yields a slightly larger slope of 0.23 .

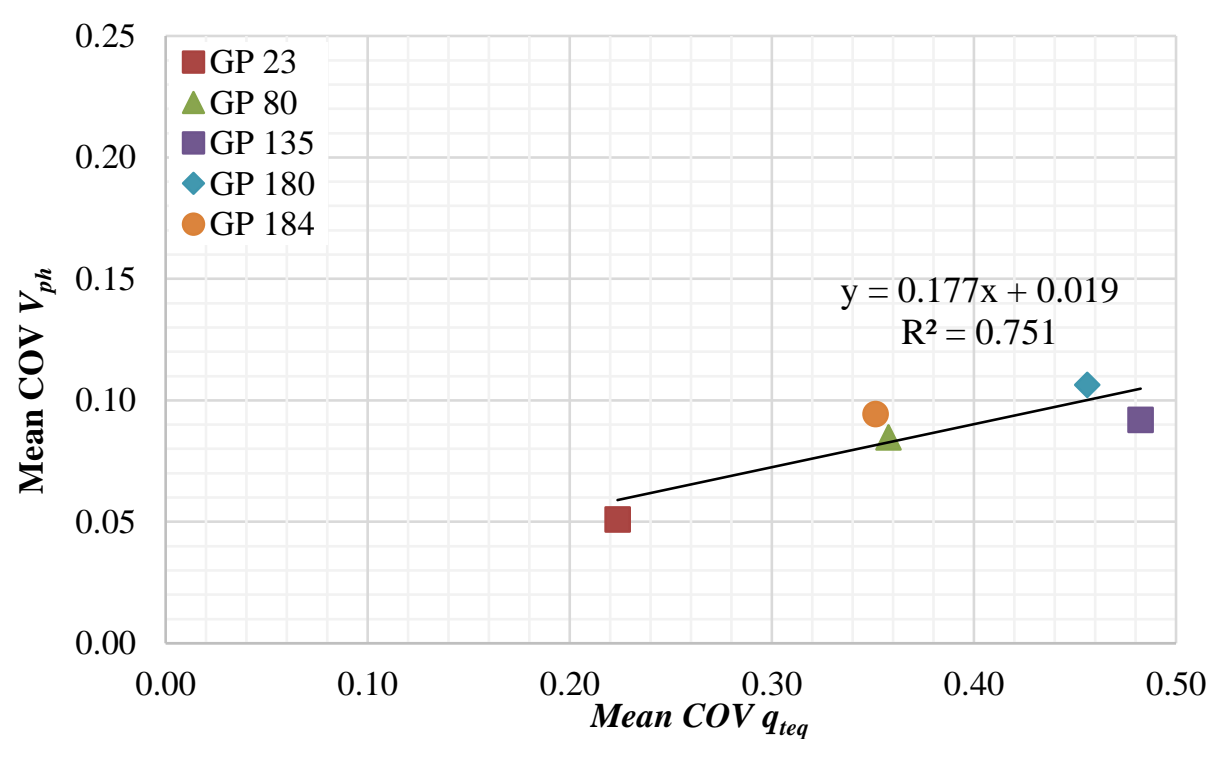

Figure 5.25 - Mean $\operatorname{COV} V_{p h}$ vs Mean $\operatorname{COV} q_{t e q}$. 
Several modifications to the data were made in an attempt to improve the EWM regression analysis. These modifications include removal of outlier data such as that of Points 30, 147A, and 192 as well as exclusion of COVs for $\lambda$ of 2.5 through $7.5 \mathrm{ft}$. Of all attempted modifications, the only case yielding a p-value less than 0.05 occurs when all of Point 147A as well as COVs for $\lambda$ of 2.5 and $5 \mathrm{ft}$ for all points are excluded (Figure 5.26). In this case, a regression analysis yields a $\mathrm{R}^{2}$ of 0.961 , a slope of 0.192 , an intercept of 0.013 , and a p-value of 0.003 .

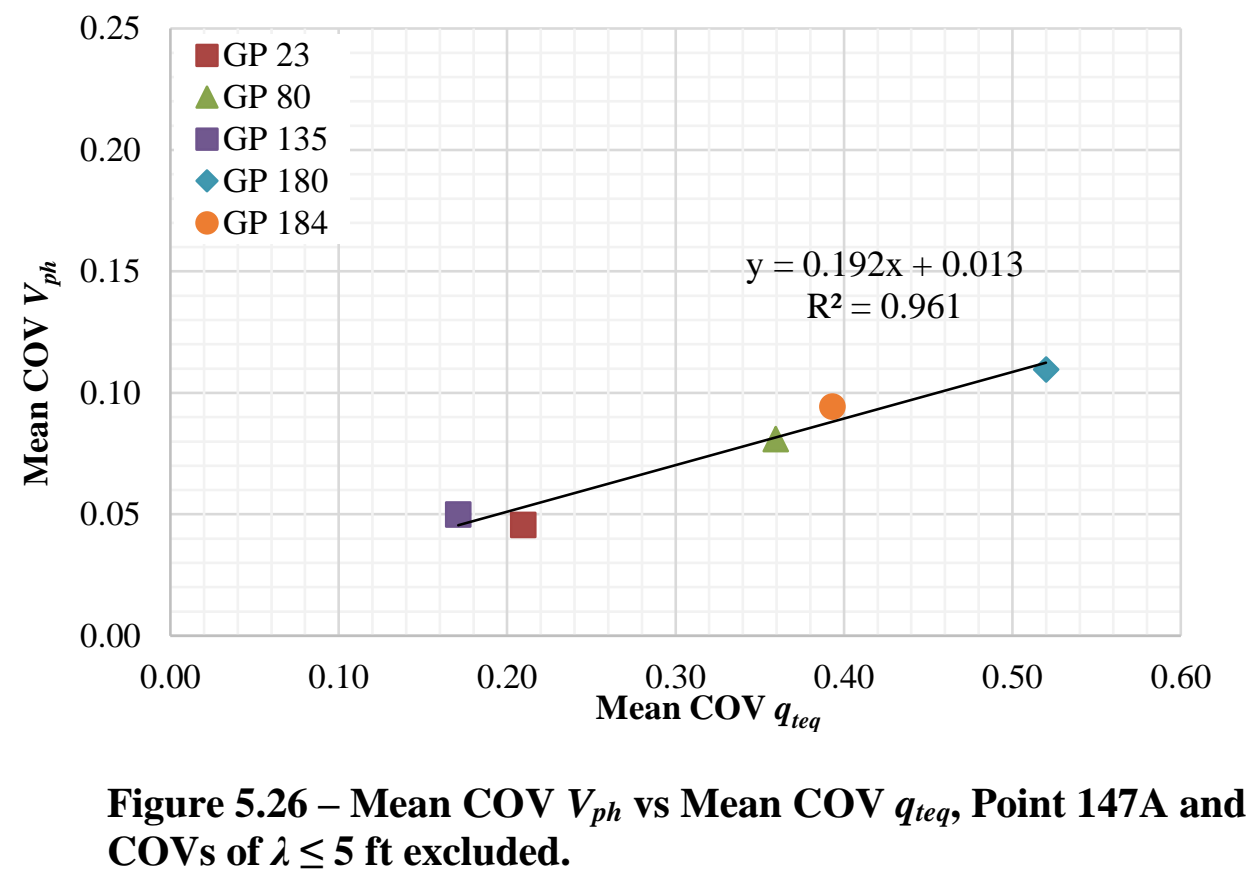


Table 5.1 - Summary of variability correlation results.

\begin{tabular}{|c|c|c|c|c|c|c|}
\hline \multirow{2}{*}{ Group } & \multicolumn{2}{|c|}{$\begin{array}{c}\text { Preliminary } \\
\text { Analysis }\end{array}$} & \multicolumn{2}{c|}{ EWM Analysis } & \multicolumn{2}{c|}{$\begin{array}{c}\text { EWM, Point 147 \& } \\
\text { all } \lambda \leq 5 \text { ft excluded }\end{array}$} \\
\cline { 2 - 7 } & $\begin{array}{c}\text { Mean } \\
\text { COV } \\
\boldsymbol{V}_{\boldsymbol{p h}}\end{array}$ & $\begin{array}{c}\text { Mean } \\
\mathbf{C O V} \boldsymbol{q}_{\boldsymbol{t}}\end{array}$ & $\begin{array}{c}\text { Mean } \\
\mathbf{C O V} \\
\boldsymbol{V}_{\boldsymbol{p h}}\end{array}$ & $\begin{array}{c}\text { Mean } \\
\mathbf{C O V} \\
\boldsymbol{q}_{\text {teq }}\end{array}$ & $\begin{array}{c}\text { Mean } \\
\mathbf{C O V} \\
\boldsymbol{V}_{\boldsymbol{p h}}\end{array}$ & $\begin{array}{c}\text { Mean } \\
\mathbf{C O V} \\
\boldsymbol{q}_{\text {teq }}\end{array}$ \\
\hline $\mathbf{2 3}$ & 0.07 & 0.35 & 0.05 & 0.22 & 0.05 & 0.21 \\
\hline $\mathbf{8 0}$ & 0.09 & 0.50 & 0.08 & 0.36 & 0.08 & 0.36 \\
\hline $\mathbf{8 4}$ & -- & 0.44 & -- & -- & -- & - \\
\hline $\mathbf{1 3 5}$ & 0.08 & 0.56 & 0.09 & 0.48 & 0.05 & 0.17 \\
\hline $\mathbf{1 8 0}$ & 0.10 & 0.47 & 0.11 & 0.46 & 0.11 & 0.52 \\
\hline $\mathbf{1 8 4}$ & 0.07 & 0.45 & 0.09 & 0.35 & 0.10 & 0.38 \\
\hline p-value & \multicolumn{2}{|c|}{0.554} & \multicolumn{2}{|c|}{0.057} & & 0.003 \\
\hline
\end{tabular}

\subsubsection{Relationship between $V_{p h}$ and $q_{t e q}$}

Utilizing the EWM to examine variability in phase velocity dispersion curves and CPT tip resistance involves the calculation of $V_{p h}$ and $q_{t e q}$ for several $\lambda$ at each test point. Plots of $V_{p h}$ vs $\lambda$ and $q_{\text {teq }}$ vs $\lambda$ for each point are provided in Appendix A. When $V_{p h}$ and $q_{t e q}$ for the same $\lambda$ are plotted, a positive and statistically significant relationship exists. This relationship is shown, in the $V_{p h}-q_{\text {teq }}$ plane, for Point 174 in Figure 5.27. The plot shows that $V_{p h}$ increases sharply relative to $q_{\text {teq }}$ across the lowest $\lambda(2.5-7.5 \mathrm{ft})$, but for $\lambda>7.5 \mathrm{ft}$ (third point from left to right), $V_{p h}$ increases consistently at a rate of about $0.1 \mathrm{ft} / \mathrm{s} / \mathrm{psi} . V_{p h}$ vs $q_{t e q}$ plots for all individual test points are provided in Appendix A and group plots of $V_{p h}$ vs $q_{t e q}$ are provided in Appendix B. 


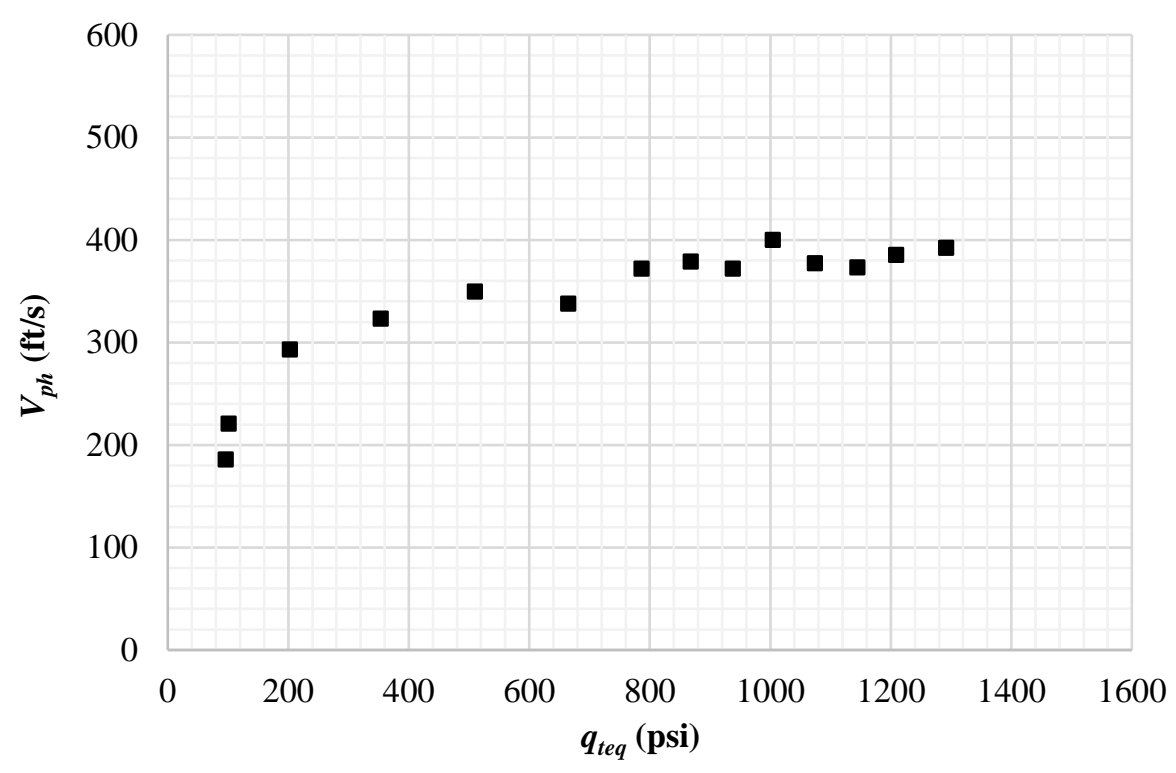

Figure 5.27 - $V_{p h}$ vs $q_{t e q}$ for Point 174.

Figure 5.28 shows that when $V_{p h}$ vs $q_{\text {teq }}$ (for the same $\lambda$ ) are plotted for all available UWL points, their relationship becomes more obvious. Inconsistency in topsoil conditions causes variability in the lowest measured $V_{p h}$ across the site, however, points tend to merge as $V_{p h}$ approaches $300 \mathrm{ft} / \mathrm{s}$ with a corresponding $q_{t e q}$ of approximately 175 psi. Figure 5.28 also shows that the gradual increase in $V_{p h}$ relative to $q_{\text {teq }}$ for $\lambda>7.5 \mathrm{ft}$ is also consistent across the UWL. When all available data are fitted with a power function, the resulting $\mathrm{R}^{2}$ is approximately 0.618 (Figure 5.28). Finally, Figures 5.29 and 5.30 show that $\mathrm{R}^{2}$ is improved when Point 147A and $\lambda \leq 5 \mathrm{ft}$ are removed from the dataset. 


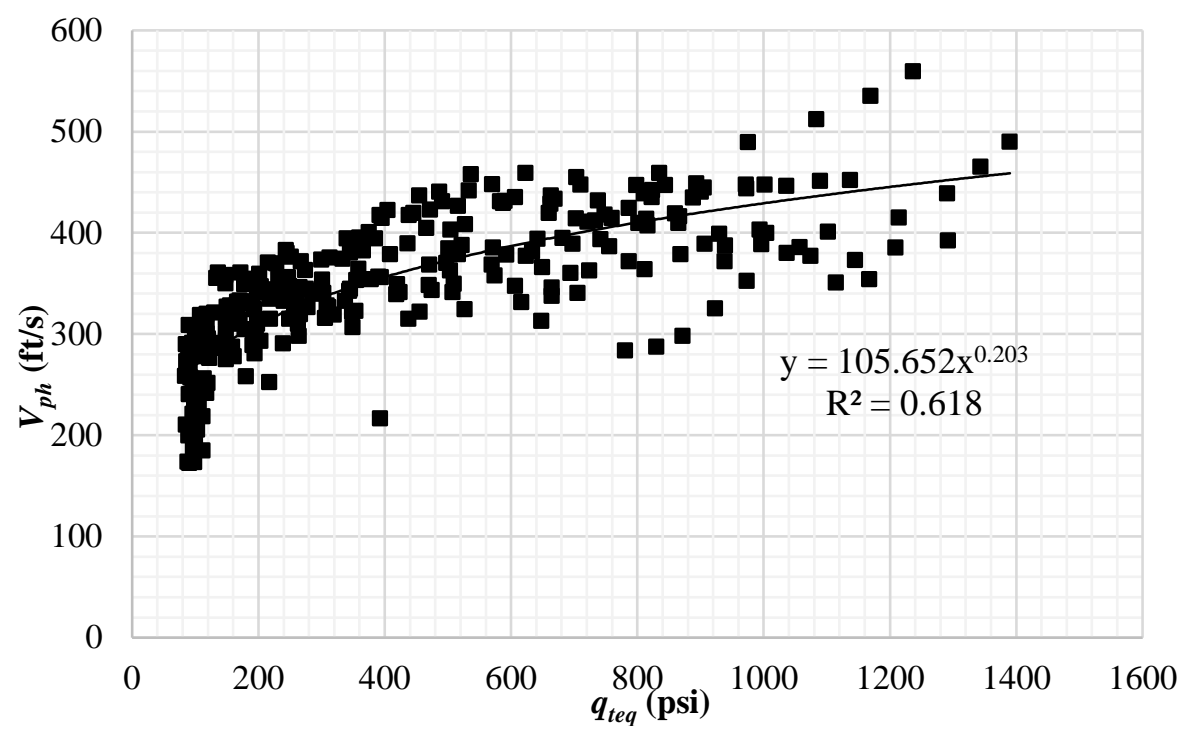

Figure 5.28 - $V_{p h}$ vs $q_{t e q}$, all available UWL points.

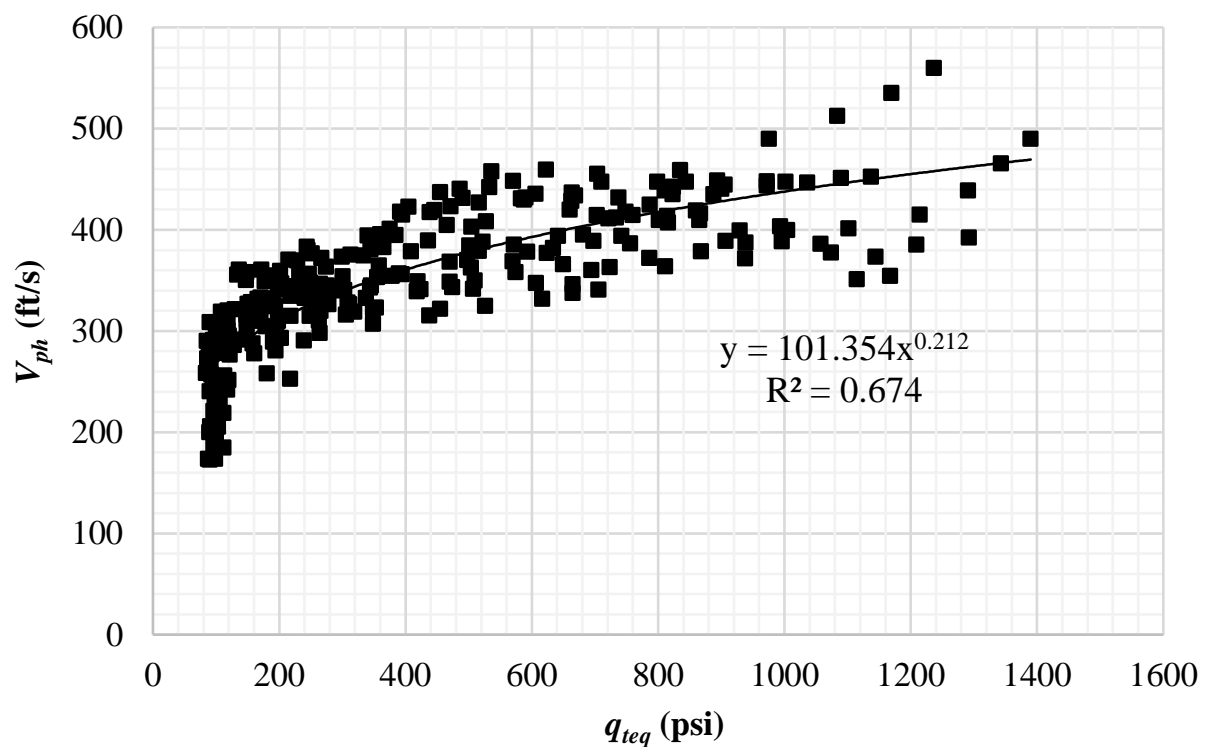

Figure 5.29 - $V_{p h}$ vs $q_{\text {teq }}$, Point 147A removed. 


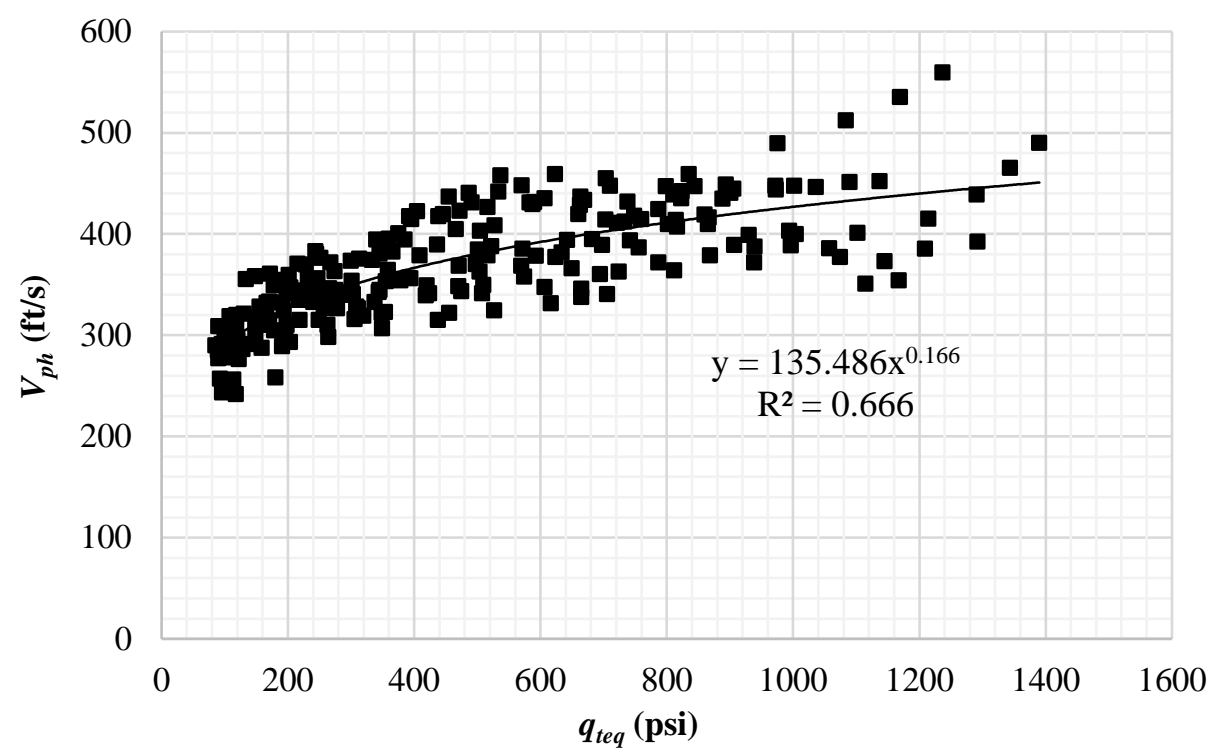

Figure $5.30-V_{p h}$ vs $q_{t e q}$, Point 147A and $\lambda \leq 5 \mathrm{ft}$ removed.

Utilizing the power functions presented in Figures 5.28, 5.29, and 5.30, it becomes possible to approximate dispersion curves from raw CPT measurements. The first step in this approximation is utilizing the EWM to transform $q_{t}$ into $q_{t e q}$ for desired $\lambda$. For each desired $\lambda$, the calculated $q_{t e q}$ and power function are then used to approximate $V_{p h}$. When the power function from Figure 5.29 is utilized to back-calculate $V_{p h}$ for all UWL test points, the mean difference between the back-calculated and measured $V_{p h}$ is less than $10 \%$. Figure 5.31 shows back-calculated and measured $V_{p h}$ for Point 194. In this case, the mean difference in measured and back-calculated $V_{p h}$ is approximately $6.5 \%$. Plots of all measured UWL dispersion curves and those back-calculated from raw CPT data utilizing this method are provided in Appendix C. 


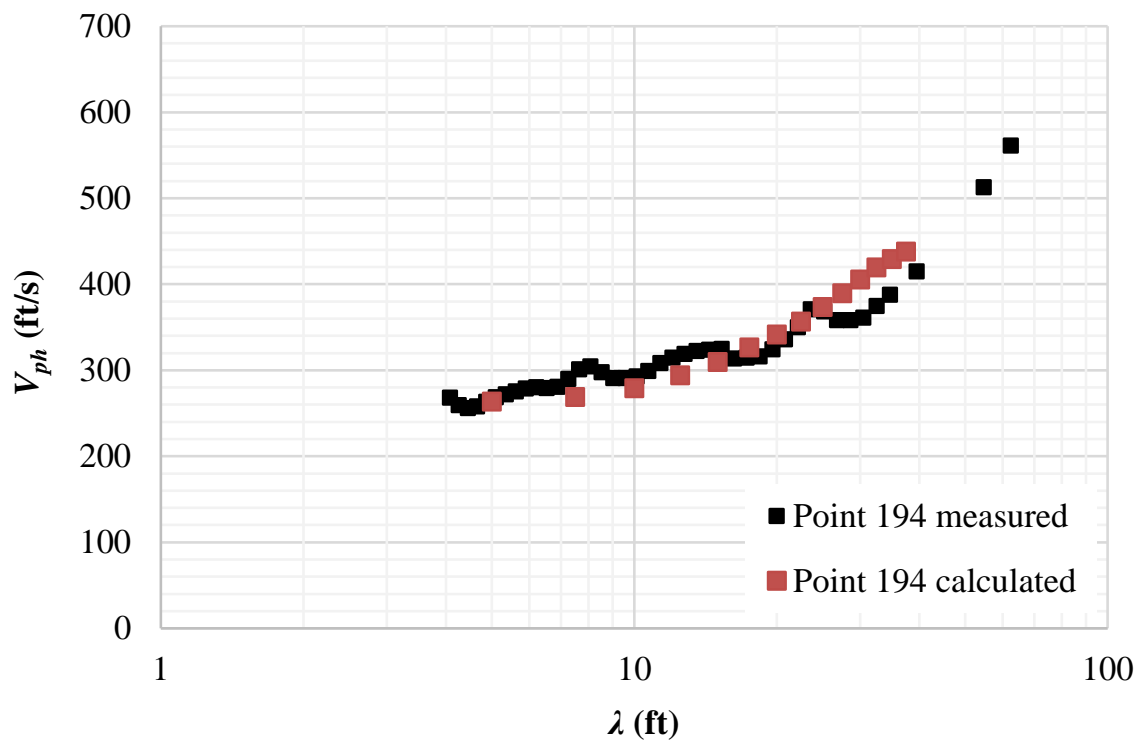

Figure 5.31 - Measured and back-calculated $V_{p h}$ for Point 194 . 


\section{Discussion}

\subsection{Overview}

While many aspects of this study warrant further discussion, this chapter covers only

those most directly related to the acceptance or rejection of the hypothesis. Section 6.2 discusses the most difficult aspect of this study: finding an appropriate method to compare variability in SASW and CPT data. The failure of the preliminary method is discussed in Section 6.3 and the success of the equivalent wavelength method (EWM) is discussed in Section 6.4. Section 6.5 covers the nature and utility of the correlation between $V_{p h}$ and $q_{t e q}$ that makes possible the estimation of SASW dispersion curves from CPT data alone. Finally, the practical and statistical limitations of this study are discussed in Section 6.7.

\subsection{Challenges in Comparing Variability of SASW and CPT Data}

Quantifying variability in CPT data relative to SASW data is difficult for a number of reasons. First, CPT measurements are generally taken every couple of centimeters in depth at a relatively well-defined point in lateral space. Across the range of measurements with depth, it is reasonable to assume that any individual measurement, say $q_{t}$ at a depth of $5 \mathrm{ft}$, is only affected by the properties of the soil within a small area surrounding the measurement. This contrasts greatly to SASW where $V_{p h}$ for a particular $\lambda$ is affected by the stiffness of the entire soil column over significant lateral expanses. As is shown in Table 4.3, the depth of penetration for a particular $\lambda$ is at least equal to $\lambda$ itself. Further, in the case of this study, global dispersion curves are the compilation of up to four smaller dispersion curves taken across lateral expanses ranging from 10 to $80 \mathrm{ft}$. 
Another non-trivial task is determining which CPT measurement(s) should be compared to phase velocity dispersion curves. Tip resistance $\left(q_{c}\right)$ is the most intuitive choice as it responds in the same manner as $V_{p h}$ to many of the same conditions. As mentioned in Section 2.3, both $V_{p h}$ and $q_{t}$ generally increase with confining pressure, geologic age, cementation, and overconsolidation ratio, and decrease with increasing void ratio. In practice, most correlations between $V_{s}$ and CPT measurements use a combination of $q_{c}$ and $f_{s}$ that are dependent on soil type and site-specific conditions (Table 2.1). Of the 15 equations presented in Table 2.1 , only two weight $f_{s}$ more heavily than $q_{c}$ or $q_{t}$ and only one relationship (Mayne 2006) relates $V_{s}$ to $f_{s}$ alone. Given the wide range of relationships between $V_{s}, q_{c}$, and $f_{s}$, comparing variability in $V_{s}$ and some combination of $q_{c}$ and $f_{s}$ would be dependent on the chosen $q_{c}-f_{s}$ combination. This study therefore only considers variability in $V_{s}$ as compared to variability in $q_{t}$ or $f_{s}$ individually. Finally, with respect to using $q_{t}$ or $q_{c}$, Wair et al. (2012) recommend that when pore pressure measurements are available, $q_{t}$ should be used in lieu of $q_{c}$.

\subsection{Failure of the Preliminary Method}

The preliminary method was the first attempt at overcoming the limitations and the fundamental differences between CPT and SASW measurements. Based on the results presented in Section 5.4, it is clear the preliminary method yields no discernable correlation between variability in CPT and SASW data. In this case, only the slightest indication of a potential correlation exists between $V_{p h}$ and $f_{s}$ while virtually no indicators of a correlation exist between $V_{p h}$ and $q_{t}$. Though it is interesting that a slightly more positive correlation occurs in comparing $f_{s}$ to $V_{p h}$, neither relationship is statistically significant and the 
disparity is most likely caused by one of the following: randomness of the data or less fluctuation in $f_{s}$ (relative to $q_{t}$ ) about the trend with depth.

While several mechanisms contribute to the failure of the preliminary method, the root cause is that relatively large fluctuations in CPT measurements are not reflected in SASW dispersion curves. This disparity is reflected in the data as roughly half of the CPT $q_{t}$ vs $z$ soundings have variability of more than 1500 psi over only a couple of feet in depth (Appendix A). This contrasts greatly to SASW dispersion curves which increase and decrease much more consistently. The difference is predominantly due to the fact that CPT soundings are very susceptible to variations in stiffness that occur over very small areas in space. Such variations in stiffness cause tremendous fluctuations in CPT data, but cannot be detected globally with SASW dispersion curves.

In order to improve the preliminary analysis, variability about the trend with depth for CPT measurements must be more comparable to those of $V_{p h}$ with respect to $\lambda$. The EWM accomplishes this by transforming CPT measurements into those that are weighted in approximately the same proportions as Rayleigh wave energy with respect to depth $\left(\mathrm{CPT}_{e q}\right)$. Though the EWM is still incapable of accounting for the global nature of SASW measurements (laterally), it significantly reduces variability in CPT data. Table 5.1 shows that the mean $\mathrm{COV}$ of $q_{t}$ ranges from 0.35 to 0.56 with the preliminary method while those of $q_{\text {teq }}(\mathrm{EWM})$ range from 0.22 to 0.46 . Further, like SASW dispersion curves, fluctuations in $\mathrm{CPT}_{e q}$ are much more gradual than those of raw CPT data.

The second major improvement of the EWM is that it uses the same number of measurements in the calculation of mean COVs. In the preliminary method, COVs are taken for all available depths (CPT) and wavelengths (SASW) where three or more values 
are available. Therefore, mean COVs of SASW incorporate around 35 data points whereas those of CPT are taken from only 10 to 15 . In converting CPT measurements to $\mathrm{CPT}_{e q}$ for the same $\lambda$ from SASW testing, each mean COV is based on the same number of data points and a more equitable comparison is achieved.

Another problem with the preliminary method is that there is no way to ensure that CPT and SASW measurements $\left(q_{t}, f_{s}, V_{p h}\right)$ are influenced by the properties of soil at the same (or similar) depths. For example, for which $\lambda$ should the COV of $V_{p h}$ be compared to the COV of $q_{t}$ taken at a depth of $6.25 \mathrm{ft}$ ? Based on the relative weighting of CPT data shown in Table 4.3, it is reasonable to say that the COV of $q_{t}$ taken at $6.25 \mathrm{ft}$ should be compared to the COV of $V_{p h}$ for $\lambda$ of $12.5 \mathrm{ft}$. This is because the highest concentration of wave energy is propagated through the depth interval from 5 to $7.5 \mathrm{ft}$. However, the table also shows that only around $20 \%$ of the total energy stored in a 12.5 -ft wave propagates through the same. The EWM accounts for this problem by only comparing $V_{p h}$ and $q_{t e q}$ for the same $\lambda$ or $\lambda_{e q}$.

Comparing $V_{p h}$ and $q_{\text {teq }}$ for the same $\lambda$ is critical to achieving a fair comparison. This is especially true for $V_{p h}$ as small fluctuations in mean COVs may produce significant changes in variability correlations (Figures $5.19,5.25,5.26$ ). The extent to which the mean $\mathrm{COV}$ for a particular group changes as a function of selected $\lambda$ is exemplified by the changes in the mean COV of $V_{p h}$ between the preliminary method and the EWM. Table 5.1 shows that while the range in mean COV of $V_{p h}$ is essentially the same in both cases, the mean COV of $V_{p h}$ for individual groups fluctuates by as little as $10 \%$ and as much as $28 \%$. Furthermore, the mean COV of Groups 23 and 80 increases while the mean COV of Groups 135,180 , and 184 decreases. The relative order of group variability, therefore, changes 
from $184,23,135,80,180$ in the preliminary method, to $23,184,80,180,135$ in the EWM. Such significant changes in the mean $\operatorname{COV}$ of $V_{p h}$, merely as a result of selected $\lambda$, underscores the importance of a consistent and equitable method of selection.

\subsection{Variability Correlation with the EWM}

Utilizing the EWM and including all available data, the correlation between the mean COV of $V_{p h}$ and the mean COV of $q_{\text {teq }}$ yields an $\mathrm{R}^{2}$ of 0.757 and a p-value of 0.057 (Figure 5.25). While the null hypothesis cannot be rejected in this case, achieving a p-value of 0.057 without removing any outliers or otherwise modifying the dataset is highly indicative of a correlation. Figure 5.25 shows that the mean $\mathrm{COV}$ of $V_{p h}$ increases at a rate of about 0.18 relative to the mean $\mathrm{COV}$ of $q_{\text {teq }}$ and the intercept is approximately 0.02 . Furthermore, the $95 \%$ confidence interval $(\mathrm{CI})$ on the intercept ranges from -0.05 to 0.09 and includes the theoretical intercept of zero.

When the dataset is improved by removal of Point $147 \mathrm{~A}$ as well as $V_{p h}$ and $q_{\text {teq }}$ from $\lambda$ of 2.5 and $5 \mathrm{ft}$ for each test, the regression is markedly improved. In this case, the null hypothesis can be rejected and a statistically significant correlation between the variability in $V_{p h}$ and $q_{t e q}$ for the same $\lambda$ does exist. The regression analysis shown in Figure 5.26 yields a p-value of 0.003 , an $\mathrm{R}^{2}$ of 0.961 , and a slope of 0.19 . In this case the $95 \% \mathrm{CI}$ on the intercept also includes the origin and ranges from -0.012 to 0.037 .

\subsubsection{Exclusion of Point 147A and $\lambda \leq 5 \mathrm{ft}$}

An examination of Figure 6.1 shows that CPT soundings at Points 147 and 147A measure a very stiff inclusion ( $q_{t}$ of approximately $1800 \mathrm{psi}$ ) between 5 and $10 \mathrm{ft}$ below the 
ground surface. While there are other cases with similarly high $q_{t}$ at shallow depths (Points $30,123,180,192)$, what makes Point 147 A particularly unusual is that the stiff inclusion is not reflected in SASW data. In fact, Figure B.10 shows that for $\lambda$ of 7 to $17 \mathrm{ft}, V_{p h}$ for Point $147 \mathrm{~A}$ is actually quite low relative to the rest of Group 135 . The combination of a such a high $q_{t}$ coupled with a low $V_{p h}$ make Point 147A an obvious outlier when $q_{t e q}$ vs $V_{p h}$ is plotted for the same $\lambda$ (Figure B.12). The most plausible explanation for this anomaly is that the stiff inclusion is localized and not prevalent across the span of SASW testing.

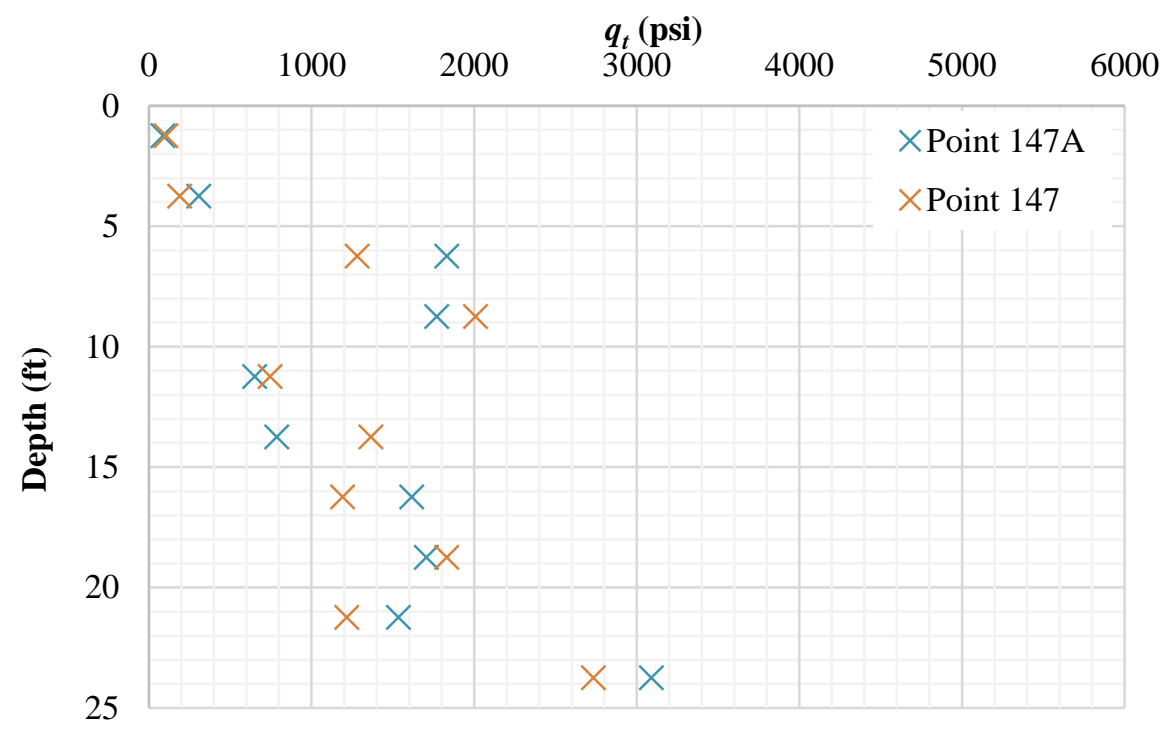

Figure 6.1 - Points 147 and 147A tip resistance $\left(q_{t}\right)$ vs depth $(\mathrm{z})$.

Regarding the exclusion of COVs taken at $\lambda$ of 2.5 and $5 \mathrm{ft}$, it is important to note that the exclusion of these COVs does not completely exclude individual measurements taken at $\lambda$ of 2.5 and $5 \mathrm{ft}$. Corrected tip resistance $\left(q_{t}\right)$ taken at depths from 0 to $6.25 \mathrm{ft}$ still influence $q_{\text {teq }}$ for $\lambda$ of $7.5 \mathrm{ft}$ or greater. The same concept applies to Rayleigh waves and $V_{p h}$ as stiffness in upper soil layers still influence $\lambda$ of $7.5 \mathrm{ft}$ or longer. The exclusion of $V_{p h}$ 
and $q_{\text {teq }}$ for $\lambda$ of 2.5 and $5 \mathrm{ft}$, in this case, serves as hedge against variability associated with fluctuating topsoil conditions at the UWL site.

\subsection{Correlation of $V_{p h}$ and $q_{t e q}$ for the same $\lambda$}

An additional result of this study is the development of a correlation between $V_{p h}$ and $q_{\text {teq }}$ for the same $\lambda$. For the UWL site, this correlation facilitated the estimation of phase velocity dispersion curves from $q_{t}$ within $10 \%$ of those measured (Section 5.5.2). This relationship is valuable because existing correlations only relate $q_{t}$ to $V_{s}$ (Table 2.1) and are particularly sensitive to local fluctuations in $q_{t}$. This can be problematic in the cases where cobbles, clasts, lenses, or other local inclusions cause significant changes in $q_{t}$ but are generally insignificant with respect to overall seismic site response. The EWM mitigates the effect of such fluctuations by weighting $q_{t}$ proportionally to Rayleigh wave energy with depth and thereby decreases the relative value of any single $q_{t}$ measurement.

Of the three equations presented in Figures 5.28, 5.29, and 5.30, the most useful for back-calculation of $V_{p h}$ from $q_{t e q}$ is that of Figure 5.29 (presented below as Equation 6.1). For this correlation, Point 147A is removed from the dataset as well as COVs calculated for $\lambda$ of 2.5 and $5 \mathrm{ft}$. The power function describing this relationship yields an $\mathrm{R}^{2}$ of 0.674 and is the best overall representation of the entire dataset. When Equation 6.1 is utilized to back-calculated dispersion curves for all associated points, the mean difference between measured and calculated dispersion curves is $9.3 \%$. A simplified version of the equation is presented as Equation 6.2. Utilizing this relationship, the mean difference between measured and back-calculated dispersion curves is $11.5 \%$. 


$$
\begin{aligned}
& V_{p h}(f t / s)=101\left(q_{t e q}(p s i)\right)^{0.212} \\
& V_{p h}(f t / s)=100\left(q_{t e q}(p s i)\right)^{0.2}
\end{aligned}
$$

A potential area of vulnerability for this relationship is what happens at longer $\lambda$ and stiffer material. This study only considers $V_{p h}$ and $q_{\text {teq }}$ for $\lambda$ up to $37.5 \mathrm{ft}$. Figures 5.28, 5.29, and 5.30 show that at long $\lambda$ and high $V_{p h}$, there are two cases where $V_{p h}$ increases sharply relative to $q_{\text {teq. }}$. The test points associated with these measurements are 121 and 184 and an examination of their dispersion curves (Figure A.42 \& A.90) show that both are fairly typical. In both cases $V_{p h}$ is in excess of $450 \mathrm{ft} / \mathrm{s}$ at $\lambda$ of $30 \mathrm{ft}$ and increasing at a rate of approximately $10 \mathrm{ft} / \mathrm{s} / \mathrm{ft}$. What is unusual, however, is that both $q_{t}$ vs $z$ plots (Figures A.43 \& A.91) have distinct stiff inclusions. Point 121 is stiff from depths of 12 to $20 \mathrm{ft}$ and Point 184 is stiff from 20 to $25 \mathrm{ft}$. These stiff inclusions, underlain by softer material, cause an increase in $V_{p h}$ that is not well-accounted for in $q_{t e q}$. However, if $q_{t}$ measurements were continued to deeper depths, $q_{t e q}$ would also increase as more energy is propagated through the stiff inclusions.

\subsection{Limitations}

The results presented in this thesis are limited in a number of ways. One of the largest limitations associated with any study is the precision and accuracy of the source measurements. The accuracy of CPT and SASW measurements are related to nature of the tests and the diligence with which they are carried out. In the case of this study, there are 
no obvious indications that any significant equipment or procedural errors were made and the results are therefore assumed to be reasonably accurate. Precision, on the other hand, can be examined in the side-by-side test results presented in Sections 5.2.1 and 5.3.1. Comparison of side-by-side CPT measurements show that differences in measured $q_{t}$ for the same depth are on average within about $20 \%$ of each other. A similar comparison of side-by-side SASW data found that $V_{p h}$ values were, on average, within about $7.6 \%$ for the same $\lambda$. Given the nature of the UWL site, which contains interbedded lenses of stiff and soft material and large particles or even cobbles, the level of precision achieved in both cases is commensurate with the nature of the measurement and material measured.

A key limitation of the variability correlations presented in this thesis are their statistical applicability. For this study, the Labadie UWL was divided into six groups and COVs for $V_{p h}$ and $q_{\text {teq }}$ are calculated with a sample size (n) of 5. Assuming lognormal distribution of $V_{p h}$ and $q_{t e q}$, the $95 \% \mathrm{CI}$ (of the COV) of $V_{p h}$ data with a COV of 0.1 ranges from approximately 0.06 to 0.3 (Verrill). Further, the $95 \%$ CI of $q_{\text {teq }}$ data with COV of 0.3 is approximately 0.17 to 0.95 (Verrill). Therefore, COVs calculated for $V_{p h}$ and $q_{t e q}$ (for a specific $\lambda$ ) may or may not be an accurate representation of the COV across the entire area of the group.

Granted, however, that COVs amongst $V_{p h}$ and $q_{\text {teq }}$ for a particular $\lambda$ are not a precise representation of the variability of the entire site, they are still a valid measure of variability amongst the five points measured. Furthermore, the mean $\mathrm{COV}$ of each group (for all $\lambda$ ) is a reasonable approximation of the collective variability between the 5 points. Finally, it should be noted that all tests were spaced in accordance with FHWA minimum guidelines for boring and sampling. Therefore, in order to increase $n$ for each calculated COV, either 
the number of tests must increase over the same area, or the area of each group must encompass more tests. The latter, of course, yields fewer data points (for the same site) in the mean $\mathrm{COV} q_{t e q}$ vs mean $\mathrm{COV} V_{p h}$ regression. 


\section{Conclusions}

\subsection{Summary}

The main goal of any geotechnical site characterization program is to reduce uncertainty in order to enhance the efficiency of geotechnical design. Ironically, however, many site characterization programs are inefficient themselves as they are often based on intrusive geotechnical testing at regular intervals. The goal of this thesis was to investigate a potential method for improving the efficiency of geotechnical site characterization programs by developing a correlation between variability in CPT and SASW measurements. Specifically, this thesis set out to test the following hypothesis: variability in SASW dispersion curves is correlated to variability in CPT measurements.

The Labadie UWL site was chosen to test the hypothesis based on its accessibility, relatively flat topography, and extensive existing CPT data. Two methods were employed to analyze CPT data obtained from Reitz \& Jens, Inc. and SASW data obtained through field testing. The first method, referred to as the preliminary method, ultimately failed to produce any meaningful relationship between variability in CPT and SASW data. Though many mechanisms were responsible for its failure, the most significant issue was that relatively large fluctuations in CPT data were not reflected in SASW dispersion curves. The second method, referred to as the EWM, overcame this shortcoming by weighting CPT measurements with respect to depth in the same manner that Rayleigh wave energy is weighted. In doing so, a measurement called the equivalent corrected tip resistances $\left(q_{\text {teq }}\right)$ was developed and calculated over the same $\lambda$ for which $V_{p h}$ measurements are available from SASW dispersion curves. 
A statistically significant correlation was achieved in comparing variability in $q_{t e q}$ to variability in $V_{p h}$ for the same $\lambda$. The first comparison, shown in Figure 5.25, yields an $\mathrm{R}^{2}$ of 0.757 and a p-value of 0.057 . Though the null hypothesis could not be rejected in this case, it warranted further investigation and a few modifications to the dataset were made. After removing Point 147A as an obvious outlier, as well as $V_{p h}$ and $q_{t e q}$ from $\lambda$ of 2.5 and $5 \mathrm{ft}$ for all tests due to fluctuations in topsoil conditions, the correlation was significantly improved. The regression analysis shown in Figure 5.26 yields a $\mathrm{p}$-value of 0.003 , an $\mathrm{R}^{2}$ of 0.961 , and a slope of 0.19 . Furthermore, the $95 \% \mathrm{CI}$ on the intercept ranges from -0.012 to 0.037 and encompasses the theoretical intercept of zero.

In addition to variability correlations, an equation relating $q_{t e q}$ to $V_{p h}$ (Eq. 6.1) was developed that facilitates the estimation of dispersion curves from $q_{t}$ alone. After converting raw $q_{t}$ values to $q_{t e q}$ utilizing Table 4.3, Equations 6.1 and 6.2 facilitate the transformation of $q_{t e q}$ for a particular $\lambda$ into an estimated $V_{p h}$ for the same. Utilizing Equation 6.1 to back-calculate dispersion curves at the UWL site resulted in a mean difference of only $9.3 \%$ from those that were measured. It is therefore possible to estimate the dispersive nature of the site with reasonable confidence based solely on $q_{t}$ vs $z$ data. However, until further research confirms or rejects the findings of this study, all correlations developed herein should be used with caution and limited to the Labadie UWL site or similar Holocene Missouri River deposits.

\subsection{Recommendations for Future Research}

There are many ways the research presented in this thesis can be expanded or improved. An attempt to reproduce the results of this study at a different geotechnical site 
is the first step in validating the findings. Future research should focus on improving the EWM by increasing the number of tests for a particular site or increasing the level of resolution for each set of tests (CPT and SWM). Test resolution may be improved by including $V_{p h}$ and $q_{\text {teq }}$ for more $\lambda$ or by utilizing an enhanced SWM such as MASW. More work is also needed to validate, or invalidate, the correlation equations developed between $V_{p h}$ and $q_{\text {teq }}$, specifically Equations 6.1 and 6.2. While these correlations are best tested though extensive testing at several sites, a comparison can be made (at a similar geotechnical site) with as little as one dispersion curve and one CPT sounding. Finally, while these equations make possible the estimation of dispersion curves from $q_{t}$ alone, a more valuable use would be to develop a method wherein $q_{t}$ may be estimated from dispersion curves alone. Some exploratory research was done to this end but ultimately failed due to low resolution in $V_{p h}$ as a function of $q_{\text {teq }}$. The combination of enhanced SWM and/or a different geotechnical site may increase the plausibility of such a correlation. 


\section{References}

Cai, G., Puppala, A. J., \& Liu, S. (2014). Characterization on the correlation between shear wave velocity and piezocone tip resistance of Jiangsu clays. Engineering Geology,171, 96-103. doi:10.1016/j.enggeo.2013.12.012

Cao, Z., Wang, Y., \& Li, D. (2016). Quantification of prior knowledge in geotechnical site characterization. Engineering Geology,203, 107-116.

United States, Department of Transportation, Federal Highway Administration. (2003). Checklist and Guidelines for Review of Geotechnical Reports and Preliminary Plans and Specifications (Vol. FHWA ED-88-053). Washington, DC: National Highway Institute.

Foti, S., Comina, C., Boiero, D., \& Socco, L. (2009). Non-uniqueness in surface-wave inversion and consequences on seismic site response analyses. Soil Dynamics and Earthquake Engineering,29(6), 982-993. doi:10.1016/j.soildyn.2008.11.004

Michigan Department of Transportation, Construction \& Technology Support Area Geotechnical Services Unit. (2004). Geotechnical Investigation and Analysis Requirements for Structures. MI.

GREDELL Engineering Resources, Inc. and Reitz \& Jens, Inc. (2011). Detailed Site Investigation, Proposed Utility Waste Disposal Area, Ameren Labadie Power Plant (Rep.). Jefferson City, MO. 
Hardin, B. O., \& Drnevich, V. P. (1972). Shear Modulus and Damping in Soils: Design Equations and Curves. Journal of the Soil Mechanics Foundation Division, ASCE, SM7, 667-692.

Hussien, M. N., \& Karray, M. (2016). Shear wave velocity as a geotechnical parameter: an overview. Canadian Geotechnical Journal,53(2), 252-272. doi:10.1139/cgj-20140524

Lai, C. G., Rix, G. J., Foti, S., \& Roma, V. (2002). Simultaneous measurement and inversion of surface wave dispersion and attenuation curves. Soil Dynamics and Earthquake Engineering,22, 923-930.

Leong, E., \& Aung, A. (2012). Weighted average velocity forward modelling of Rayleigh surface waves. Soil Dynamics and Earthquake Engineering,43, 218-228. doi:10.1016/j.soildyn.2012.07.030

Leong, E. C., \& Aung, A. M. (2013). Global Inversion of Surface Wave Dispersion Curves Based on Improved Weighted Average Velocity Method. Journal of Geotechnical and Geoenvironmental Engineering, 139(12), 2156-2169. doi:10.1061/(asce)gt.1943-5606.0000939

Lew, M., Ii, K. H., Davis, C. A., Ponnaboyina, H., Hudson, M. B., \& Perry, D. L. (2012). A Comparison of SASW Survey Results with In Situ Field Investigation Methods. GeoCongress 2012. doi:10.1061/9780784412121.279 
Mayne, P. W., Christopher, B. R., \& DeJong, J. (2002). Subsurface investigationsgeotechnical site characterization: reference manual (United States, Department of Transportation, Federal Highway Administration, National Highway Institute). Washington, DC.

Mayne, P. W. (2007). Cone Penetration Testing State-of-Practice (Rep. No. NCHRP Project 20-05). Washington, DC: Transportation Research Board Synthesis Study.

Park, C. B., Miller, R. D., \& Xia, J. (1999). Multichannel analysis of surface waves. Geophysics,64(3), 800-808.

Park, C. B., Miller, R. D., Xia, J., \& Ivanov, J. (2007). Multichannel analysis of surface waves (MASW) — active and passive methods. The Leading Edge,26(1), 60-64. doi:10.1190/1.2431832

Phoon, K., \& Kulhawy, F. (1999). Characterization of geotechnical variability. Canadian Geotechnical Journal,36, 612-624.

Phoon, K., \& Kulhawy, F. (1999). Evaluation of geotechnical property variability. Canadian Geotechnical Journal,36, 625-639.

Rix, G. J., Lai, C. G., Orozco, M. C., Hebeler, G. L., \& Roma, V. (2001). Recent Advances in Surface Wave Methods for Geotechnical Site Characterization. In $X V$ International Conference on Soil Mechanics and Geotechnical Engineering, 2001. Istanbul. 
Robertson, P. K., Sasitharan, S., Cunning, J. C., \& Sego, D. C. (1996). Shear-wave velocity to evaluate in-situ state of Ottawa sand. International Journal of Rock Mechanics and Mining Sciences \& Geomechanics Abstracts,33(2). doi:10.1016/01489062(96)83957-x

Robertson, P. K., \& Cabal, K. L. (2015). Guide to Cone Penetration Testing for Geotechnical Engineering (6th ed., Rep.). Signal Hill, CA: Gregg Drilling \& Testing, Inc.

Stokoe, K. H., II, Wright, S. G., Bay, J. A., \& Roesset, J. M. (1994). Characterization of Geotechnical Sites by SASW Method. New Delhi, India: 13th International Conference on Soil Mechanics and Foundation Engineering.

Thompson, E. M., Baise, L. G., \& Kayen, R. E. (2006). Spatial Correlation of ShearWave Velocity within San Francisco Bay Sediments. GeoCongress 2006. doi:10.1061/40803(187)134

Tokimatsu, K. (1995). Geotechnical Site Characterization Using Surface Waves (Rep.). Tokyo, Japan: Tokyo Institute of Technology.

Tran, K. T., \& Hiltunen, D. R. (2012). Two-Dimensional Inversion of Full Waveforms Using Simulated Annealing. Journal of Geotechnical and Geoenvironmental Engineering,138(9), 1075-1090. doi:10.1061/(asce)gt.1943-5606.0000685

Verrill, S. (2003). Confidence Bounds for Normal and Lognormal Distribution Coefficients of Variation (Rep. No. FPL-RP-609). Madison: Department of Agriculture. 
Wair, B. R., DeJong, J. T., \& Shantz, T. (2012). Guidelines for Estimation of Shear Wave Velocity Profiles (Rep. No. PEER 2012/08). CA: Pacific Earthquake Engineering Research Center Headquarters.

Zhang, J., Huang, H., Juang, C., \& Su, W. (2014). Geotechnical reliability analysis with limited data: Consideration of model selection uncertainty. Engineering Geology,181, 27-37. doi:10.1016/j.enggeo.2014.08.002 


\section{Appendices}

\section{Appendix A: Point plots}

\section{Group 23}

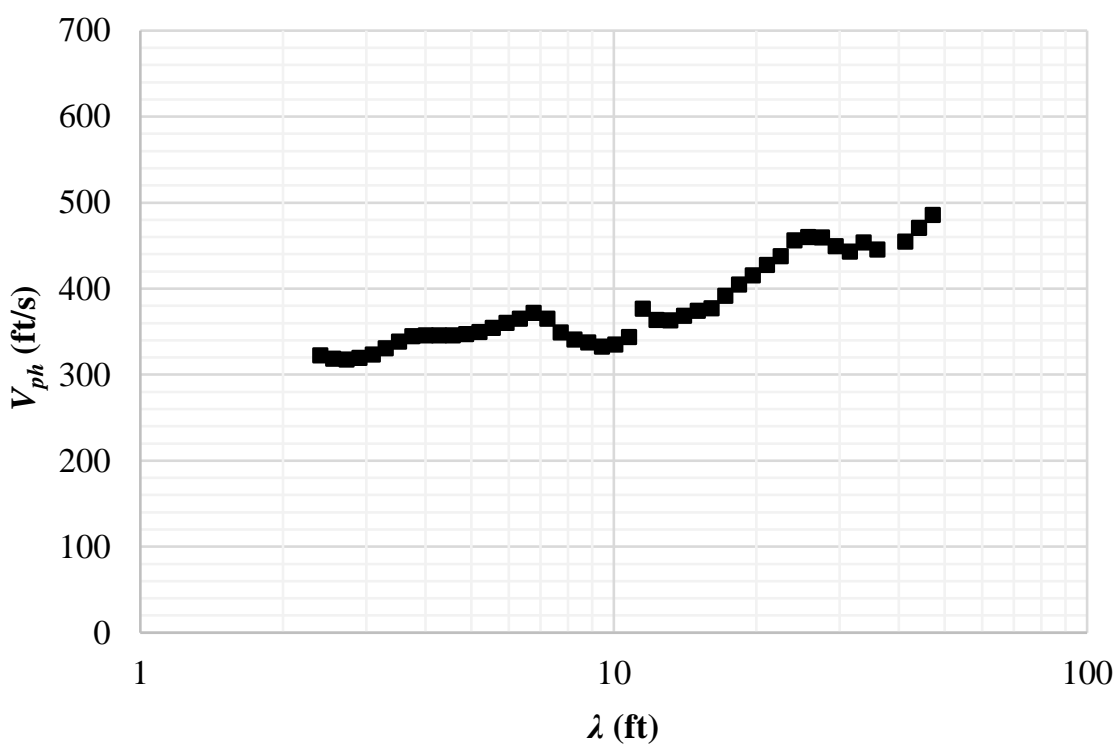

Figure A.1 - Point 16 dispersion curve.

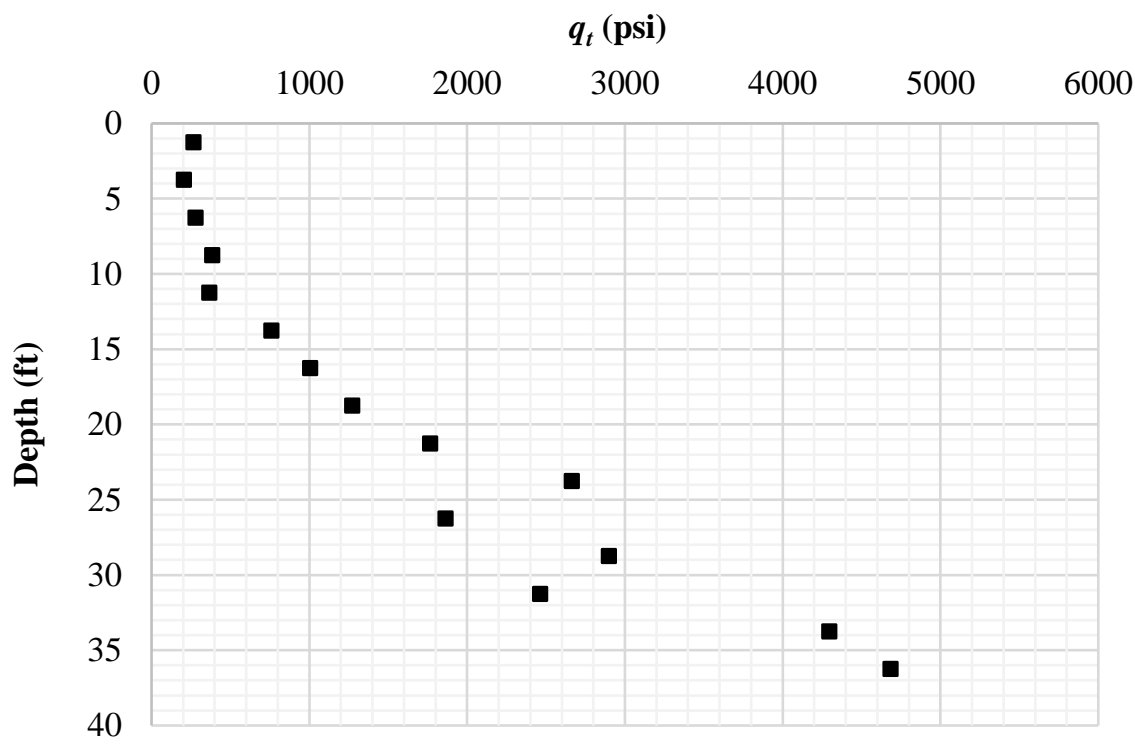

Figure A.2 - Point 16 tip resistance $\left(q_{t}\right)$ vs depth $(z)$. 


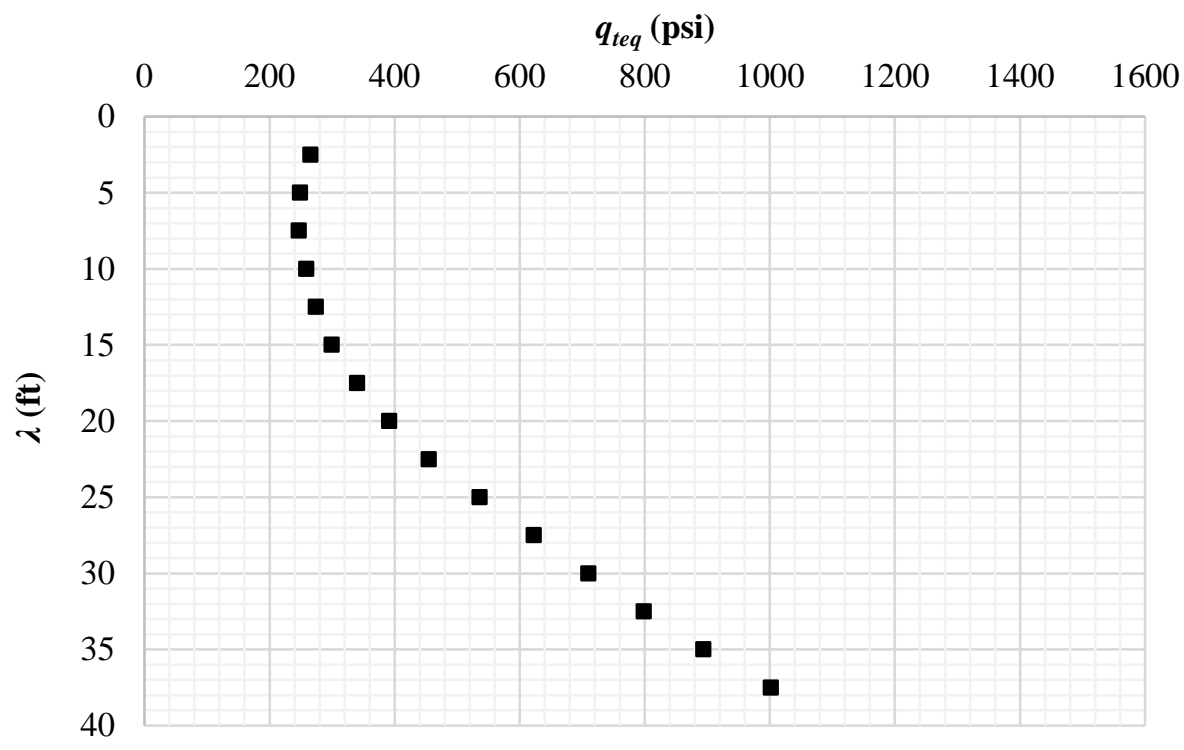

Figure A.3 - Point 16 equivalent tip resistance $\left(q_{t e q}\right)$ vs wavelength $(\lambda)$.

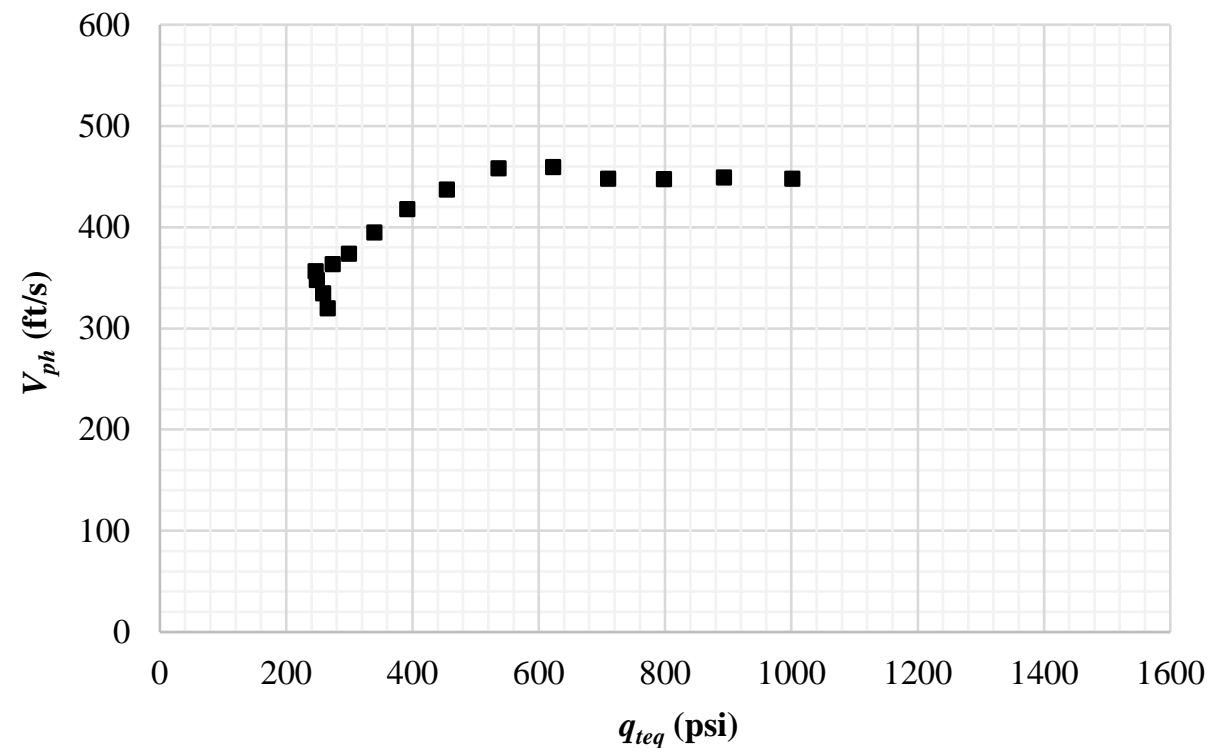

Figure A.4 - Pont 16 equivalent tip resistance $\left(q_{\text {teq }}\right)$ vs phase velocity $\left(V_{p h}\right)$ for the same $\lambda$. 


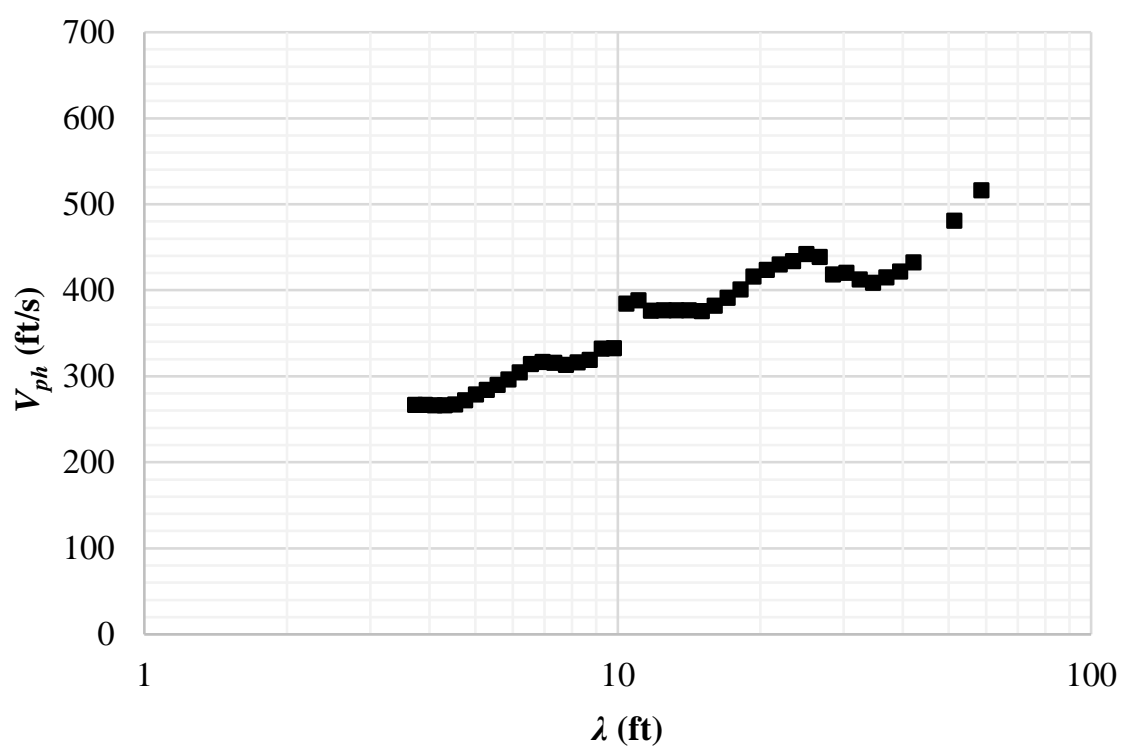

Figure A.5 - Point 18 dispersion curve.

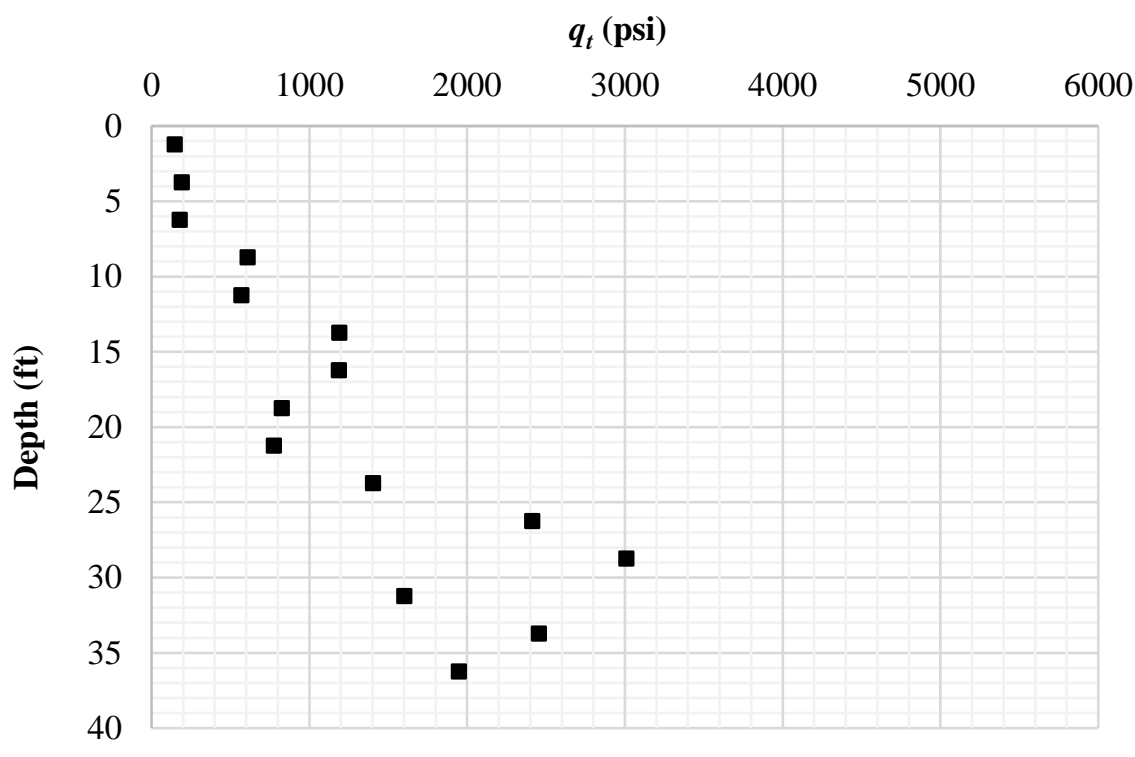

Figure A.6 - Point 18 tip resistance $\left(q_{t}\right)$ vs depth (z). 


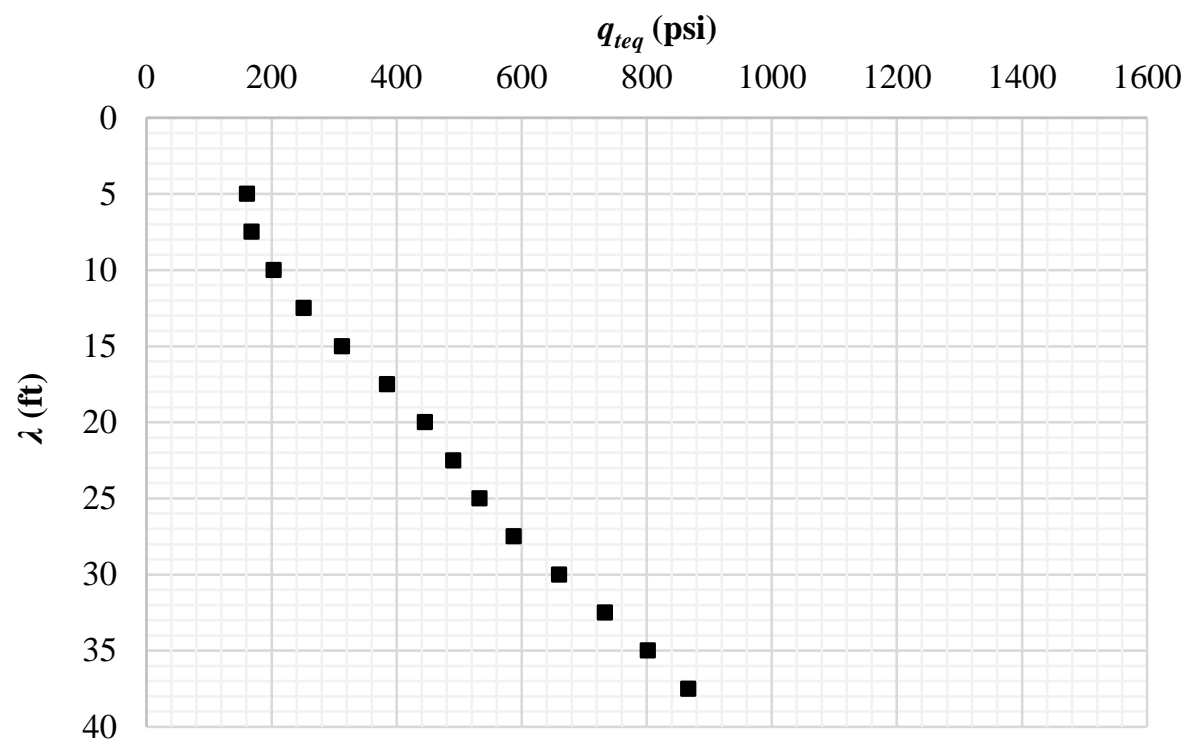

Figure A.7 - Point 18 equivalent tip resistance $\left(q_{\text {teq }}\right)$ vs wavelength $(\lambda)$.

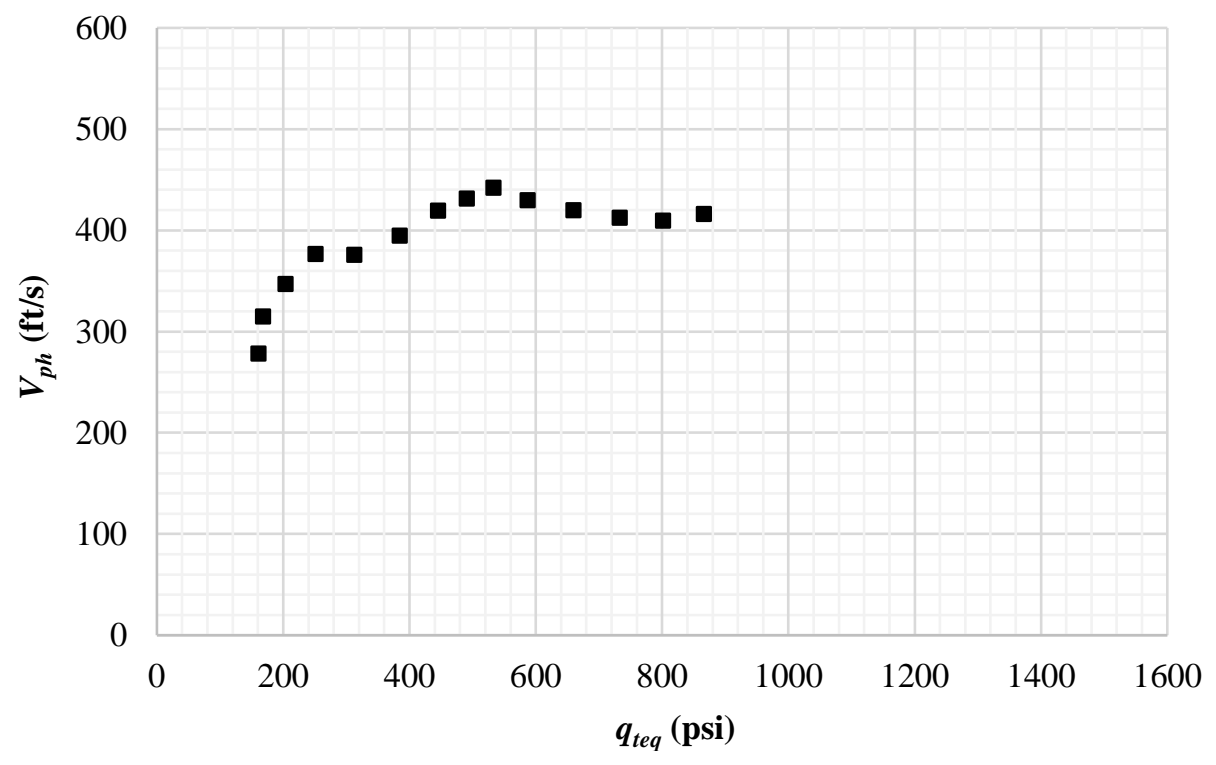

Figure A.8 - Pont 18 equivalent tip resistance $\left(q_{t e q}\right)$ vs phase velocity $\left(V_{p h}\right)$ for the same $\lambda$. 


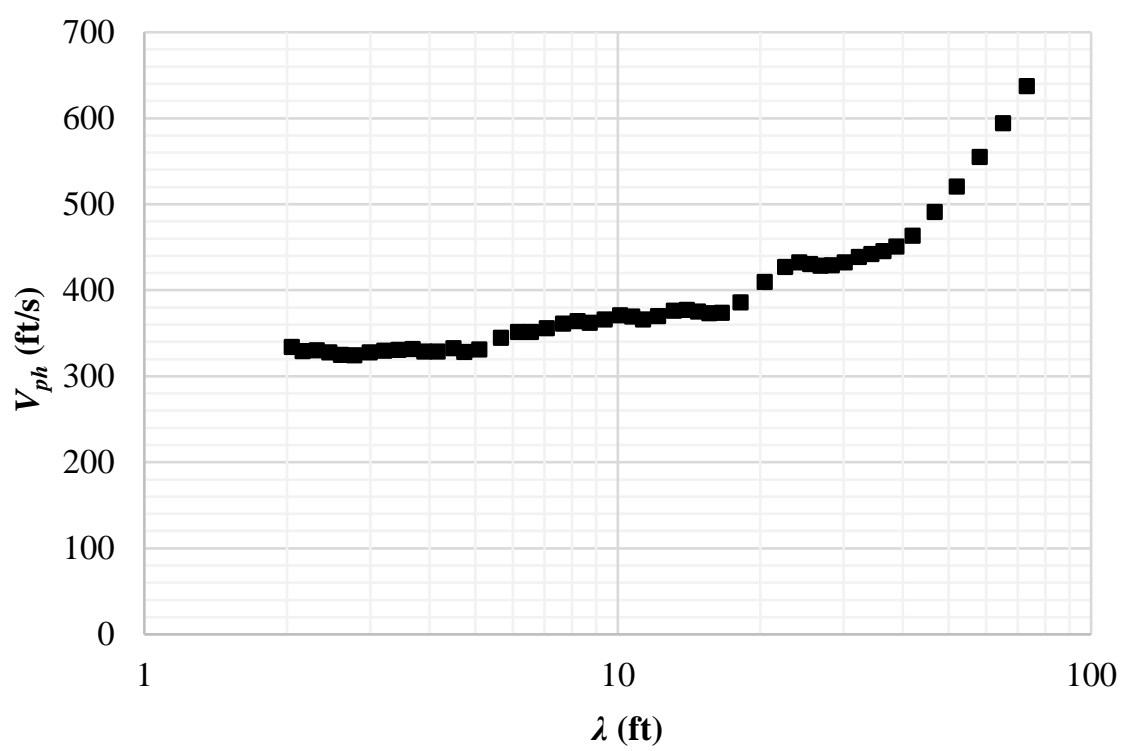

Figure A.9 - Point 23 dispersion curve.

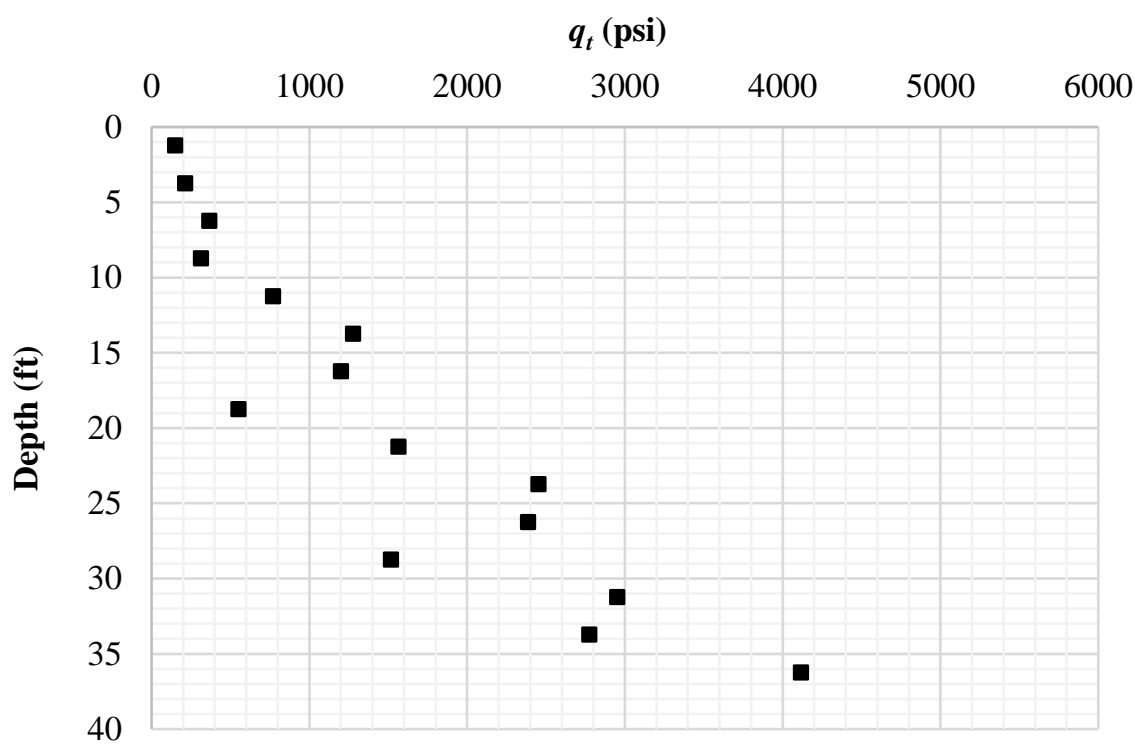

Figure A.10 - Point 23 tip resistance $\left(q_{t}\right)$ vs depth (z). 


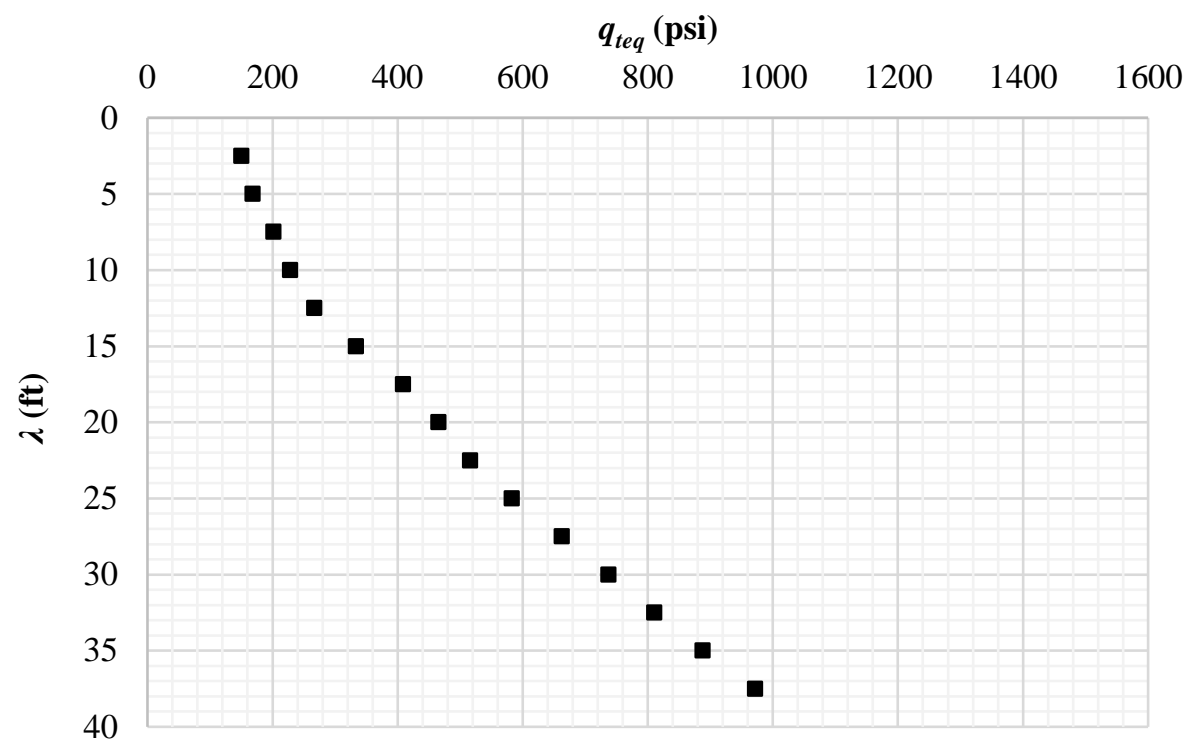

Figure A.11 - Point 23 equivalent tip resistance $\left(q_{t e q}\right)$ vs wavelength ( $\lambda$ ).

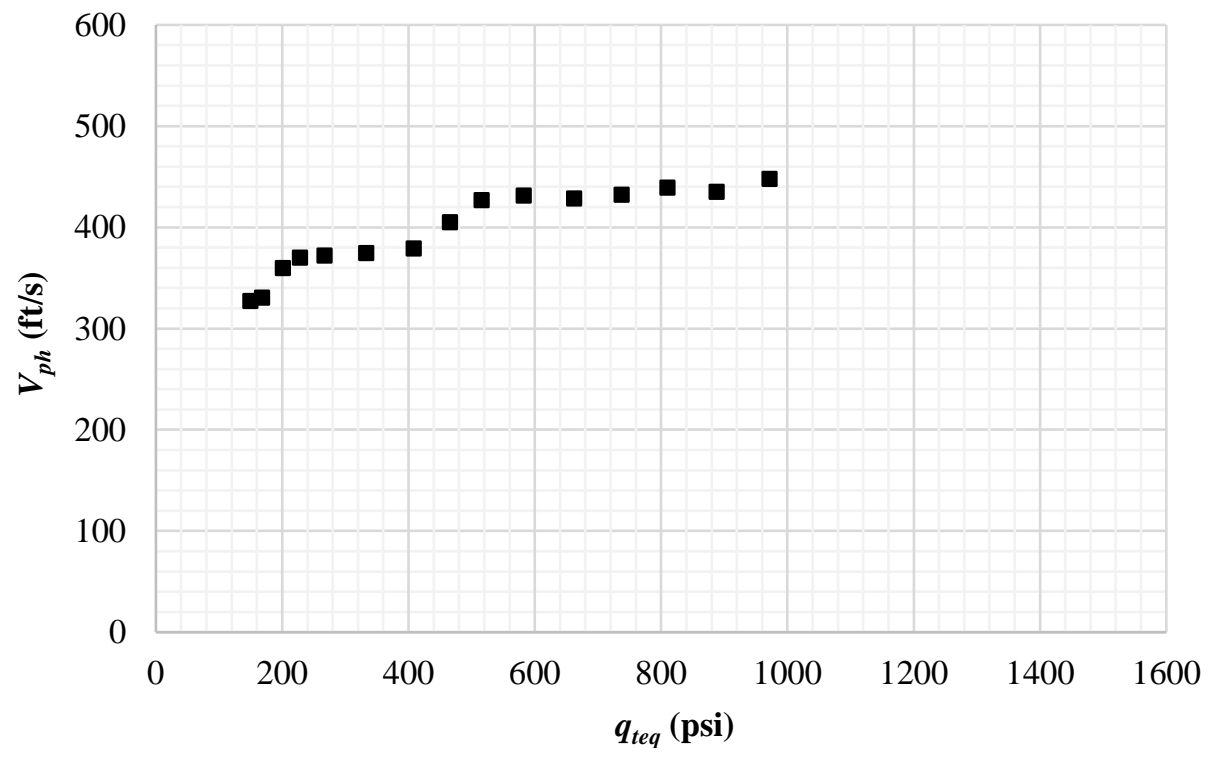

Figure A.12 - Pont 23 equivalent tip resistance $\left(q_{t e q}\right)$ vs phase velocity $\left(V_{p h}\right)$ for the same $\lambda$. 


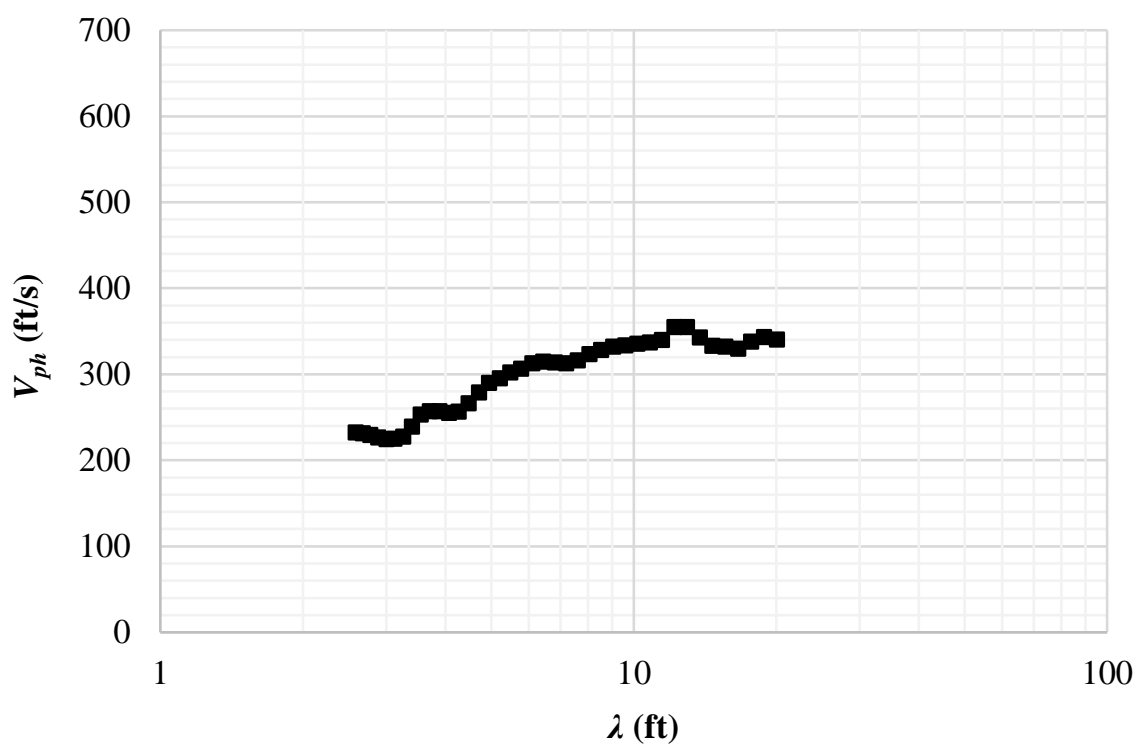

Figure A.13 - Point 28 dispersion curve.

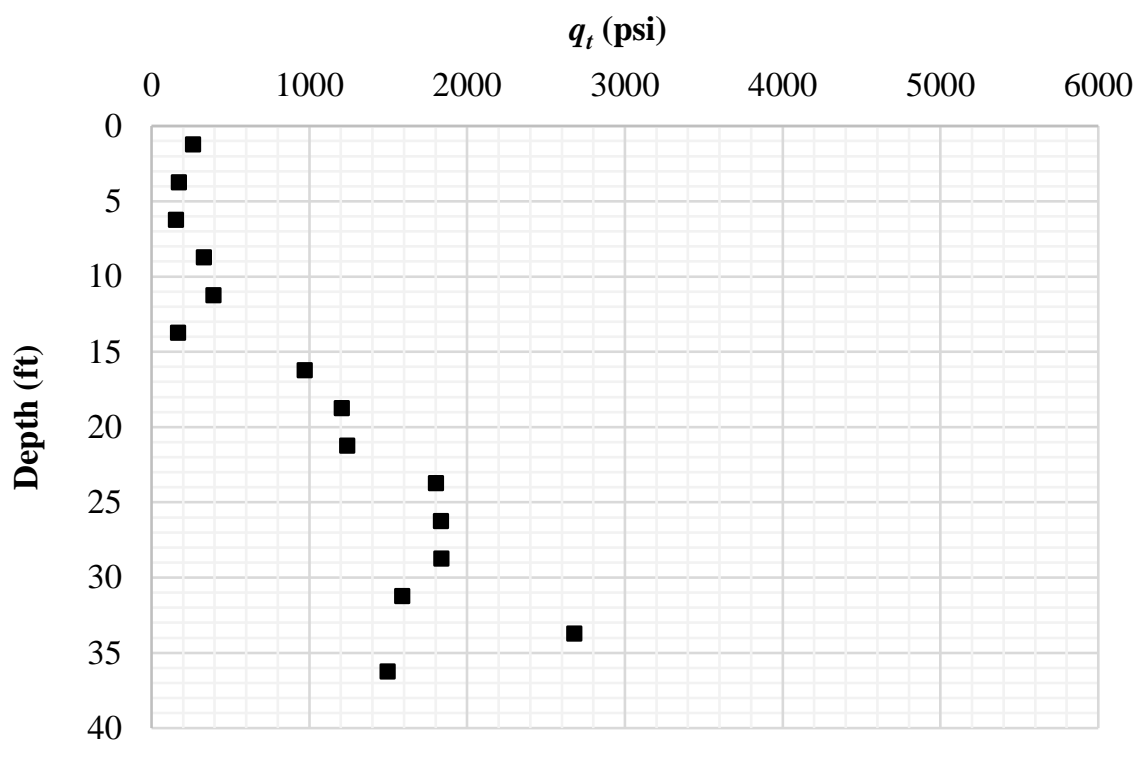

Figure A.14 - Point 28 tip resistance $\left(q_{t}\right)$ vs depth (z). 


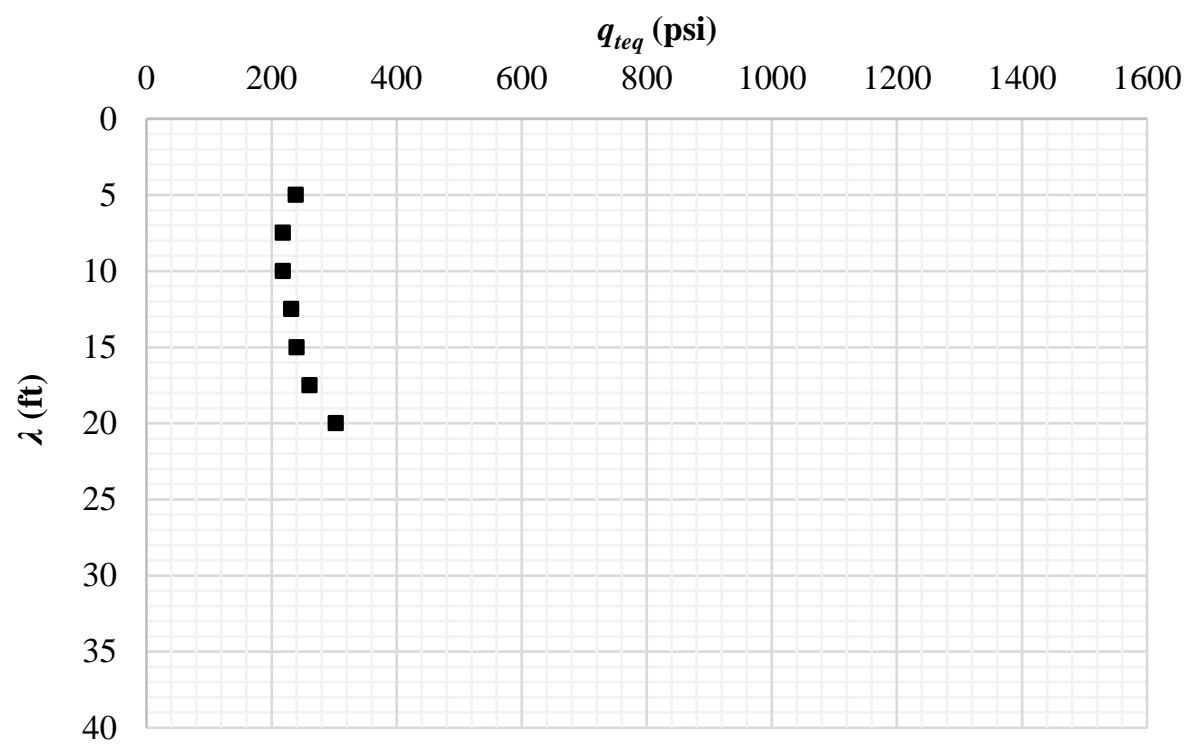

Figure A.15 - Point 28 equivalent tip resistance $\left(q_{t e q}\right)$ vs wavelength (ג).

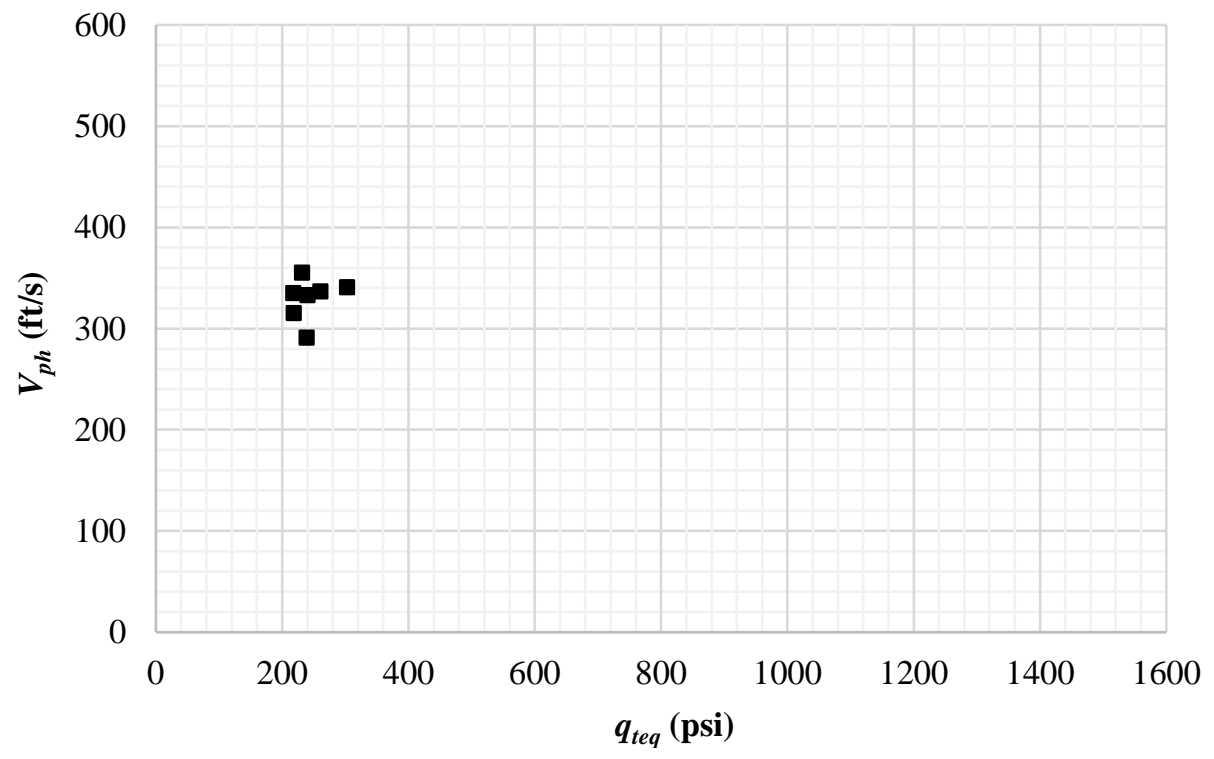

Figure A.16 - Pont 28 equivalent tip resistance $\left(q_{\text {teq }}\right)$ vs phase velocity $\left(V_{p h}\right)$ for the same $\lambda$. 


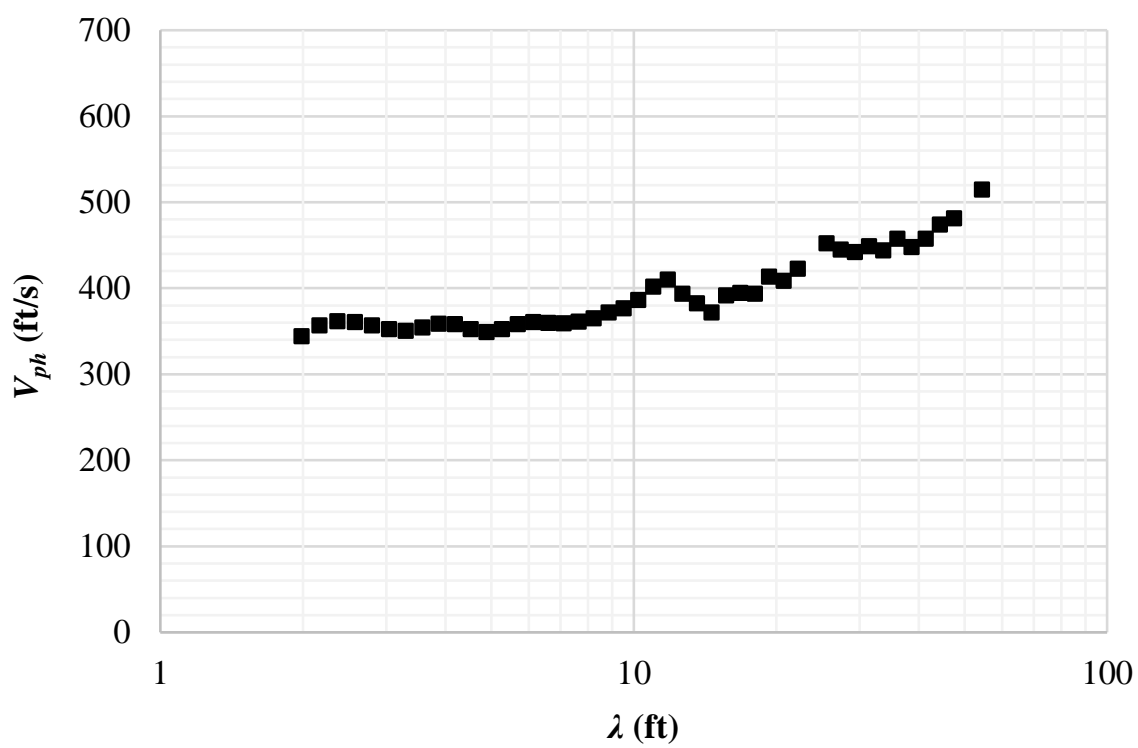

Figure A.17 - Point 30 dispersion curve.

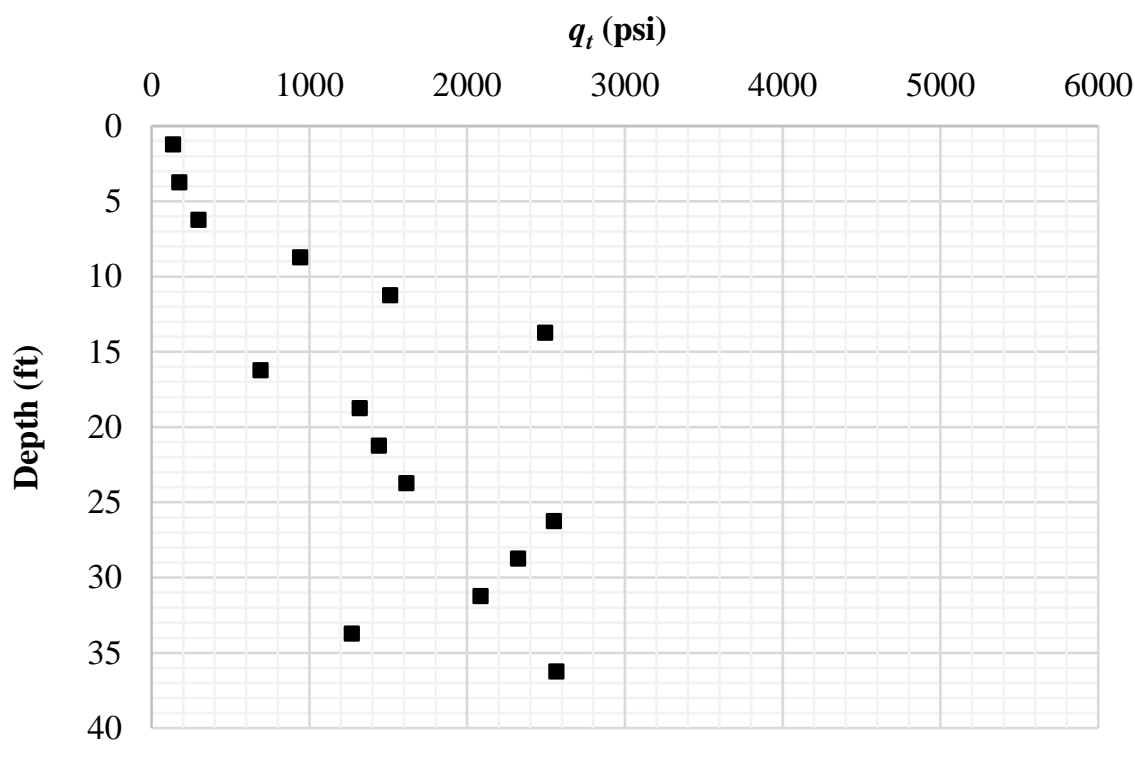

Figure A.18 - Point 30 tip resistance $\left(q_{t}\right)$ vs depth (z). 


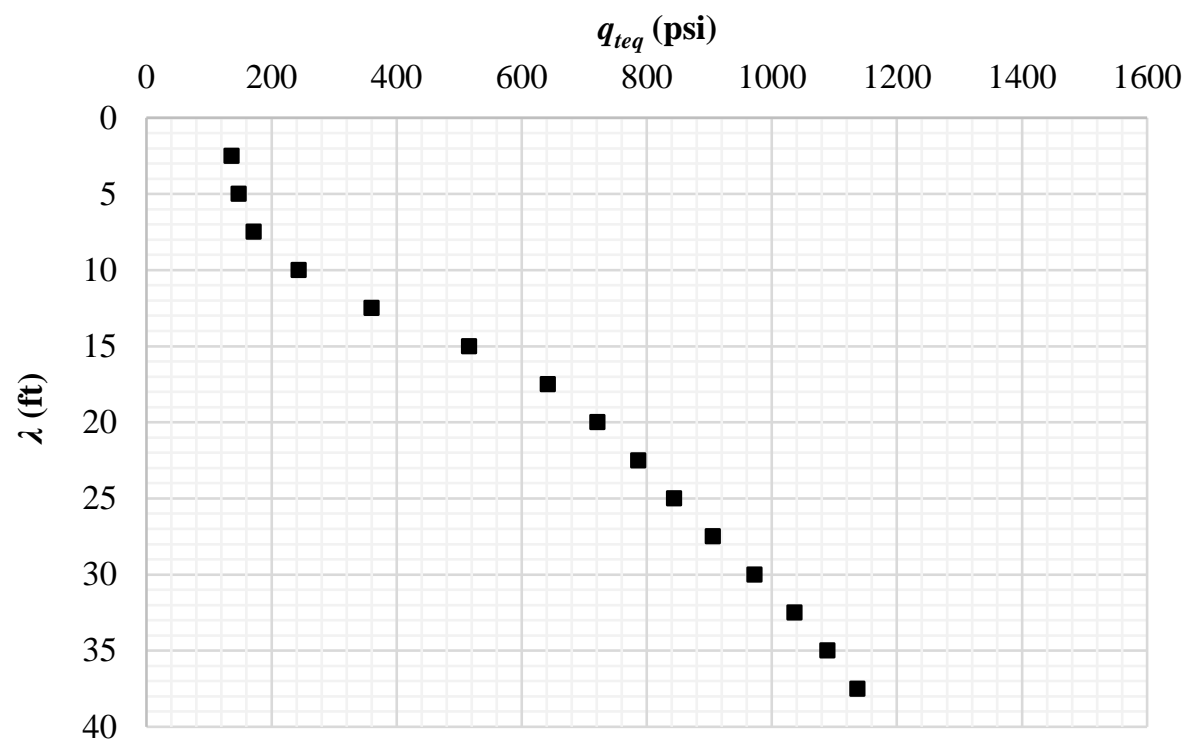

Figure A.19 - Point 30 equivalent tip resistance $\left(q_{t e q}\right)$ vs wavelength ( $\lambda$ ).

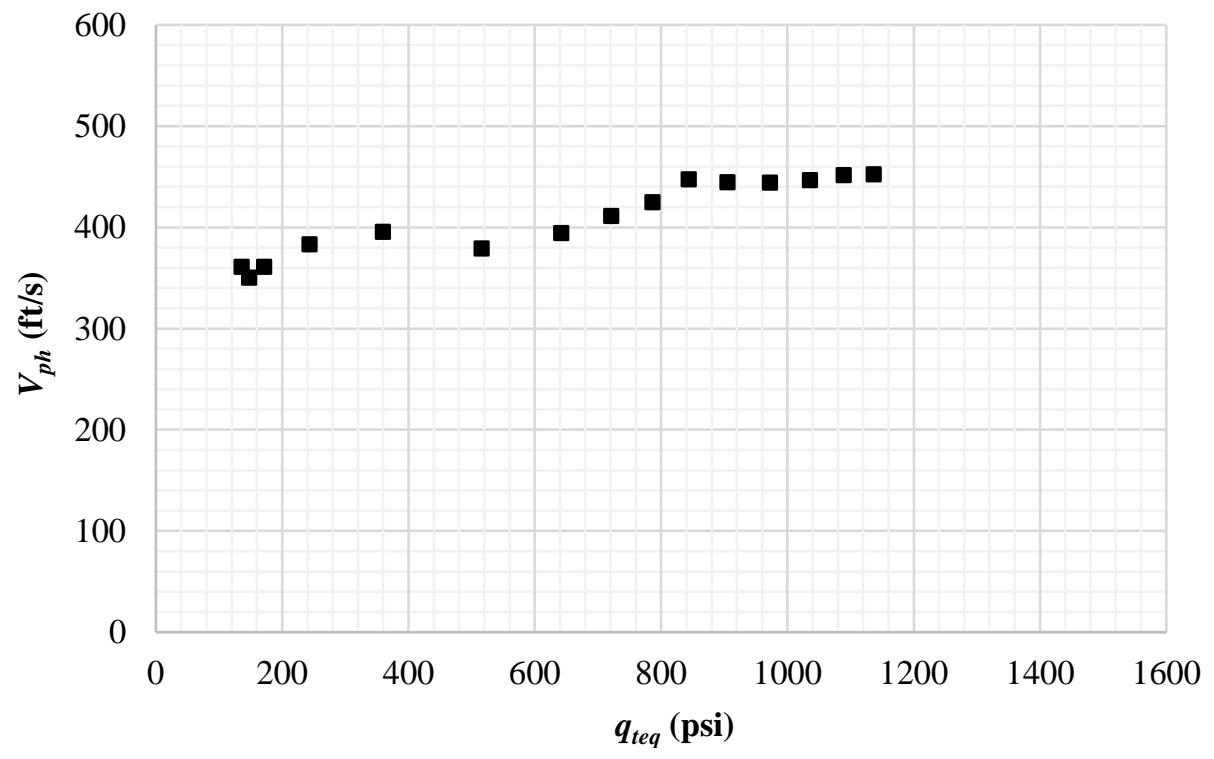

Figure A.20 - Pont 30 equivalent tip resistance $\left(q_{t e q}\right)$ vs phase velocity $\left(V_{p h}\right)$ for the same $\lambda$. 


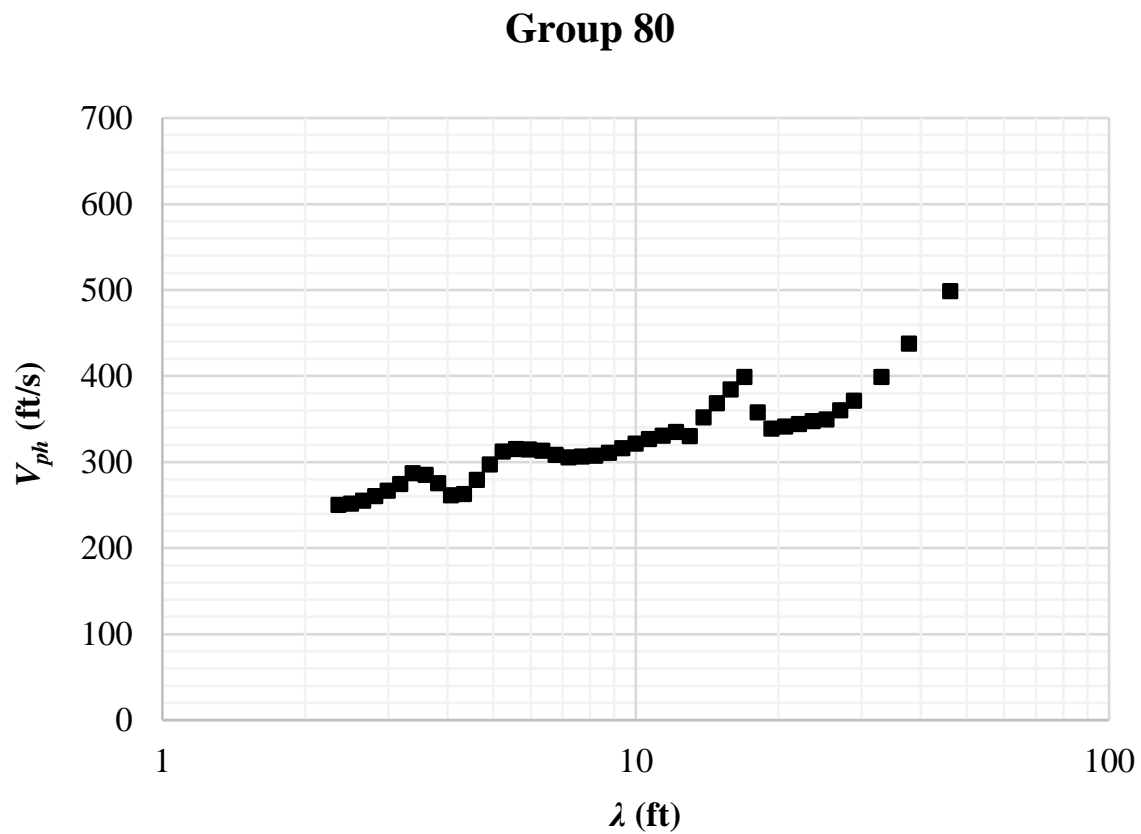

Figure A.21 - Point 64 dispersion curve.

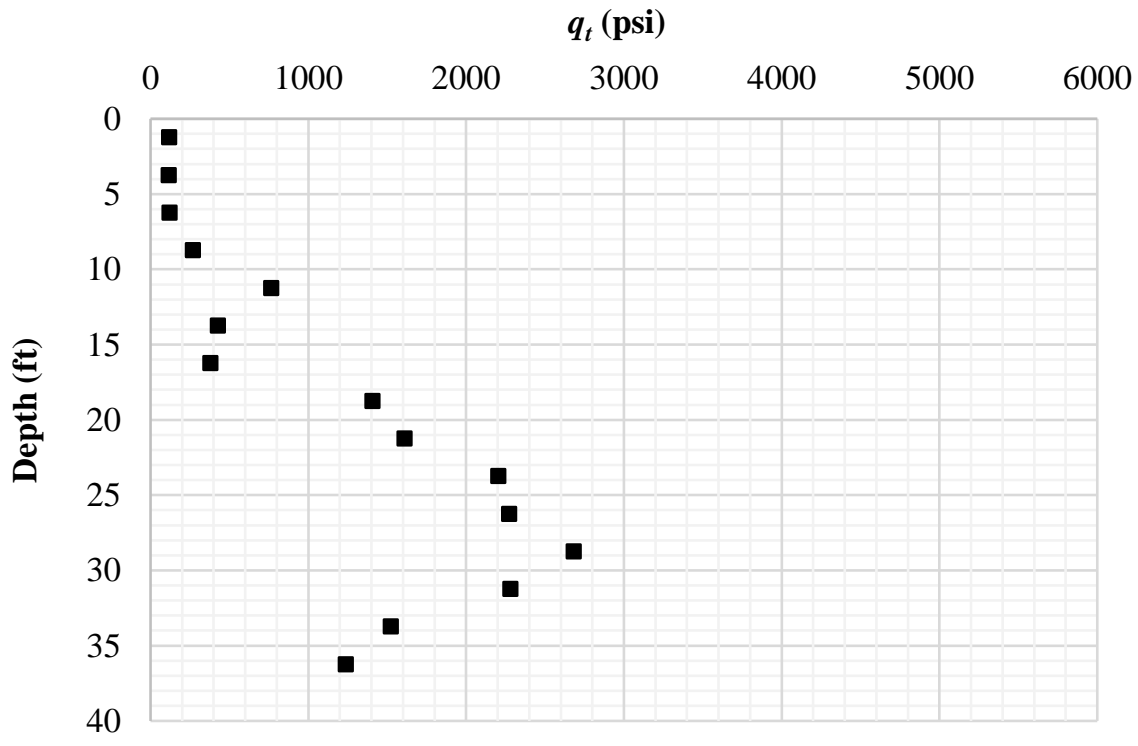

Figure A.22 - Point 64 tip resistance $\left(q_{t}\right)$ vs depth $(z)$. 


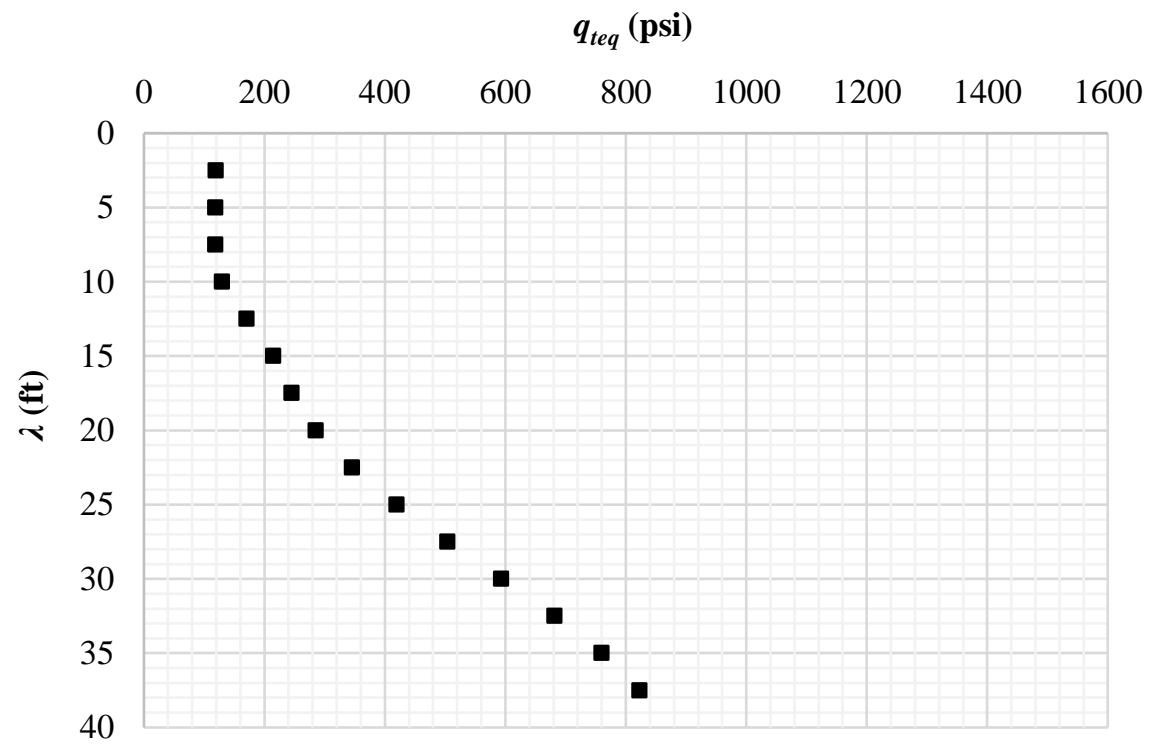

Figure A.23 - Point 64 equivalent tip resistance $\left(q_{\text {teq }}\right)$ vs wavelength (ג).

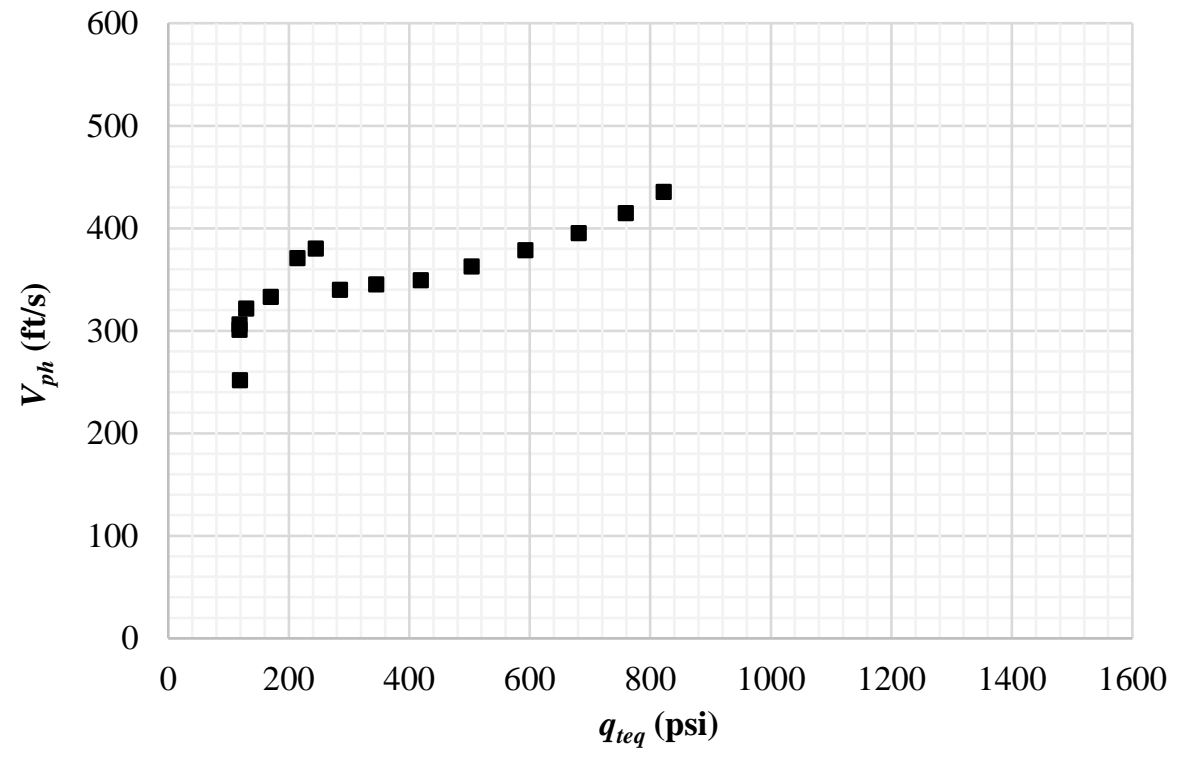

Figure A.24 - Pont 64 equivalent tip resistance $\left(q_{\text {teq }}\right)$ vs phase velocity $\left(V_{p h}\right)$ for the same $\lambda$. 


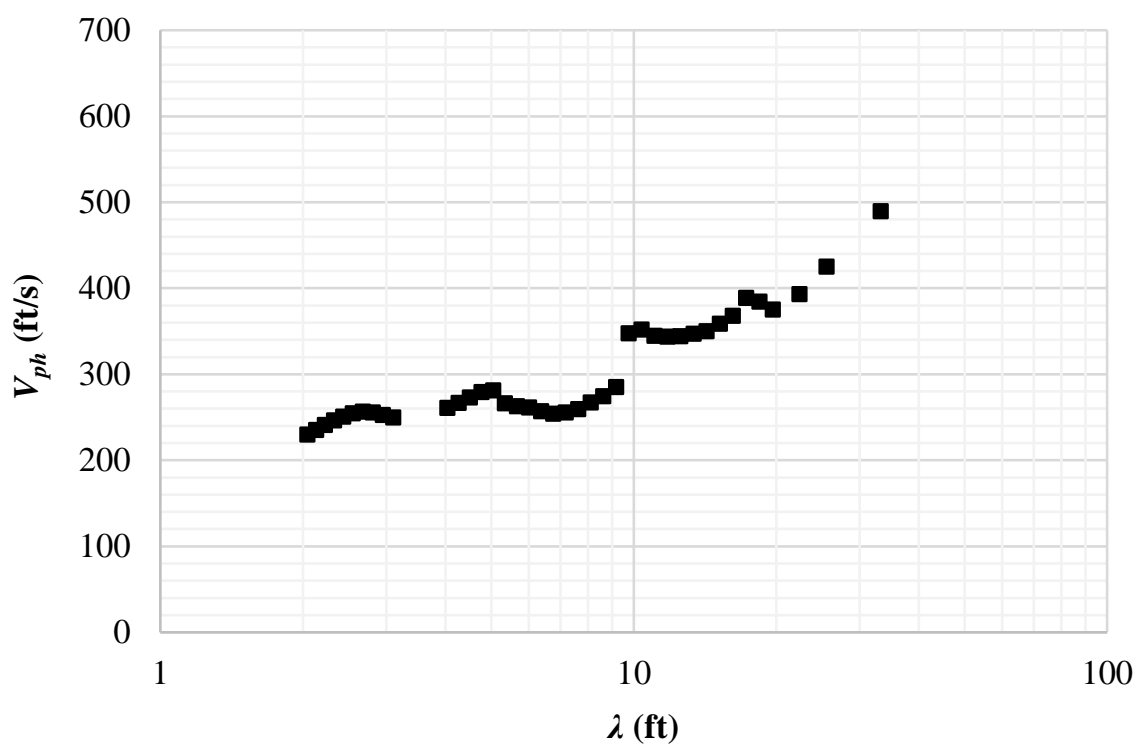

Figure A.25 - Point 66 dispersion curve.

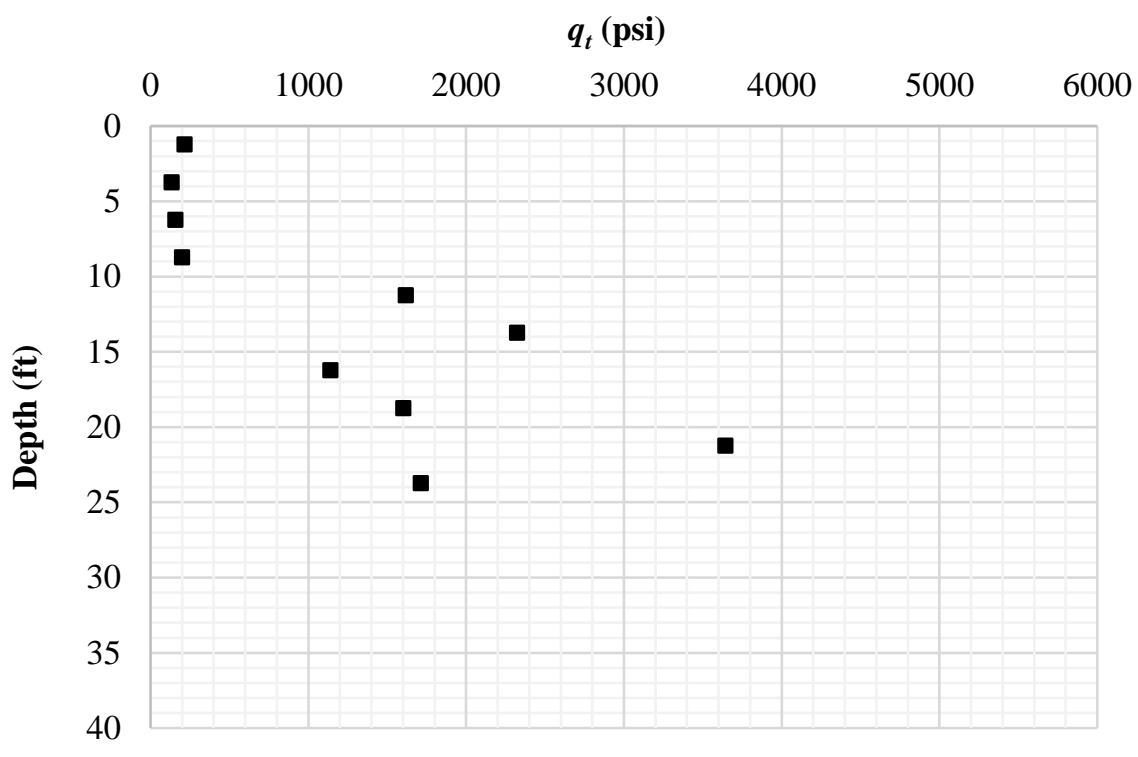

Figure A.26 - Point 66 tip resistance $\left(q_{t}\right)$ vs depth (z). 


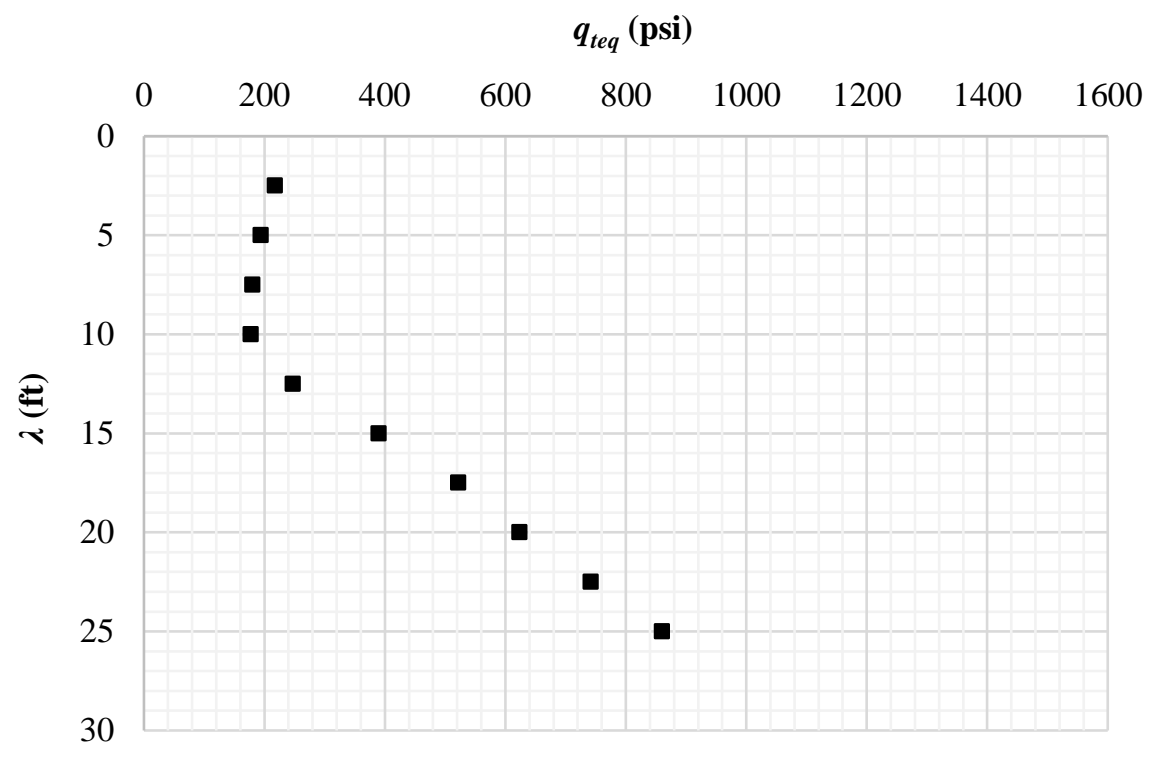

Figure A.27 - Point 66 equivalent tip resistance ( $\left.q_{t e q}\right)$ vs wavelength (ג).

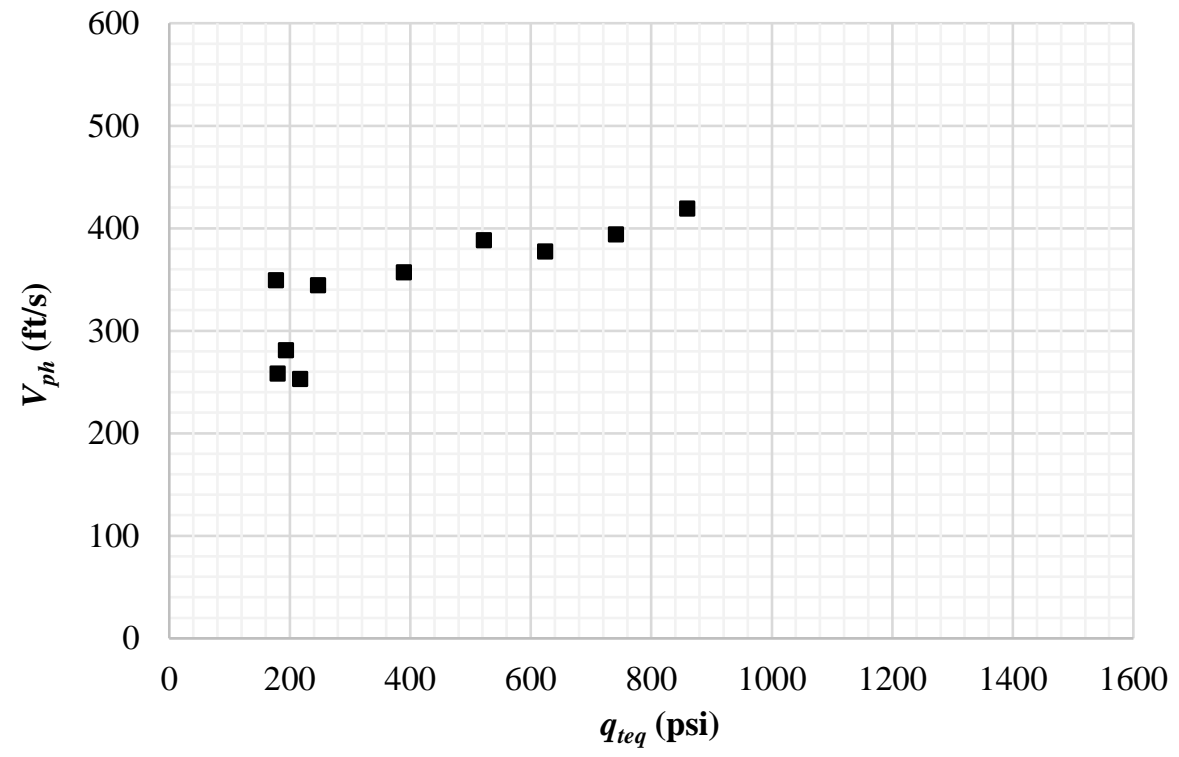

Figure A.28 - Pont 66 equivalent tip resistance $\left(q_{\text {teq }}\right)$ vs phase velocity $\left(V_{p h}\right)$ for the same $\lambda$. 


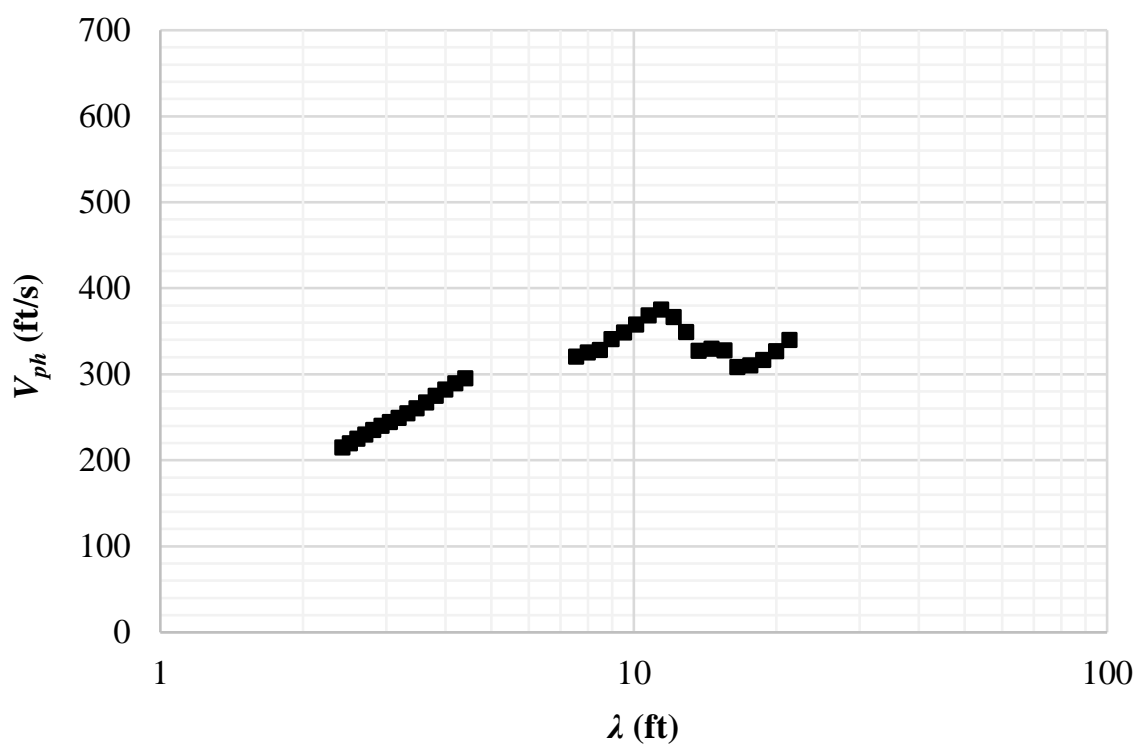

Figure A.29 - Point 80 dispersion curve.

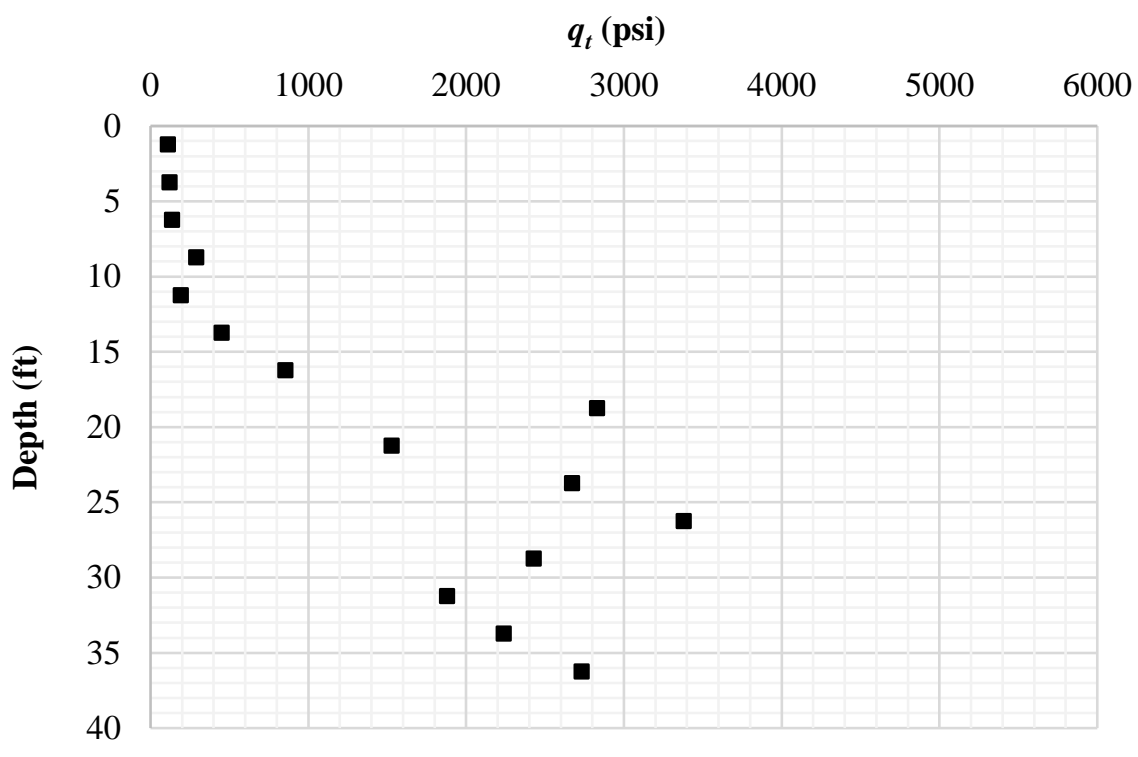

Figure A.30 - Point 80 tip resistance $\left(q_{t}\right)$ vs depth (z). 


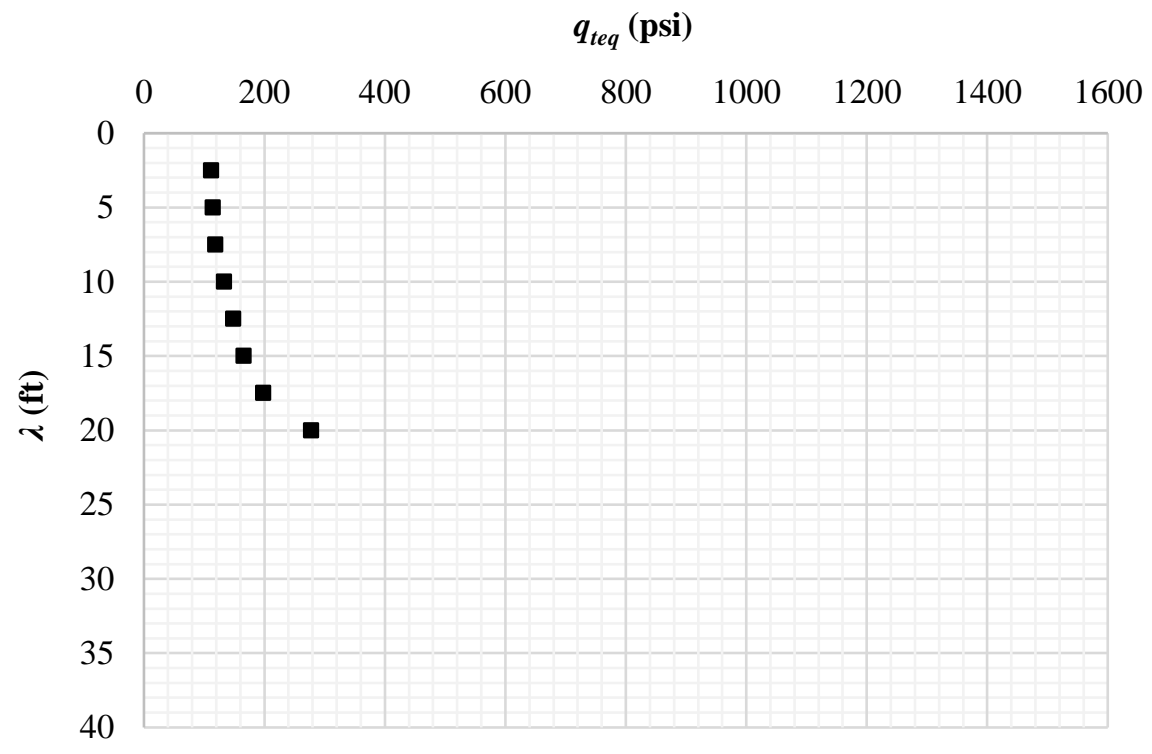

Figure A.31 - Point 80 equivalent tip resistance ( $\left.q_{t e q}\right)$ vs wavelength (ג).

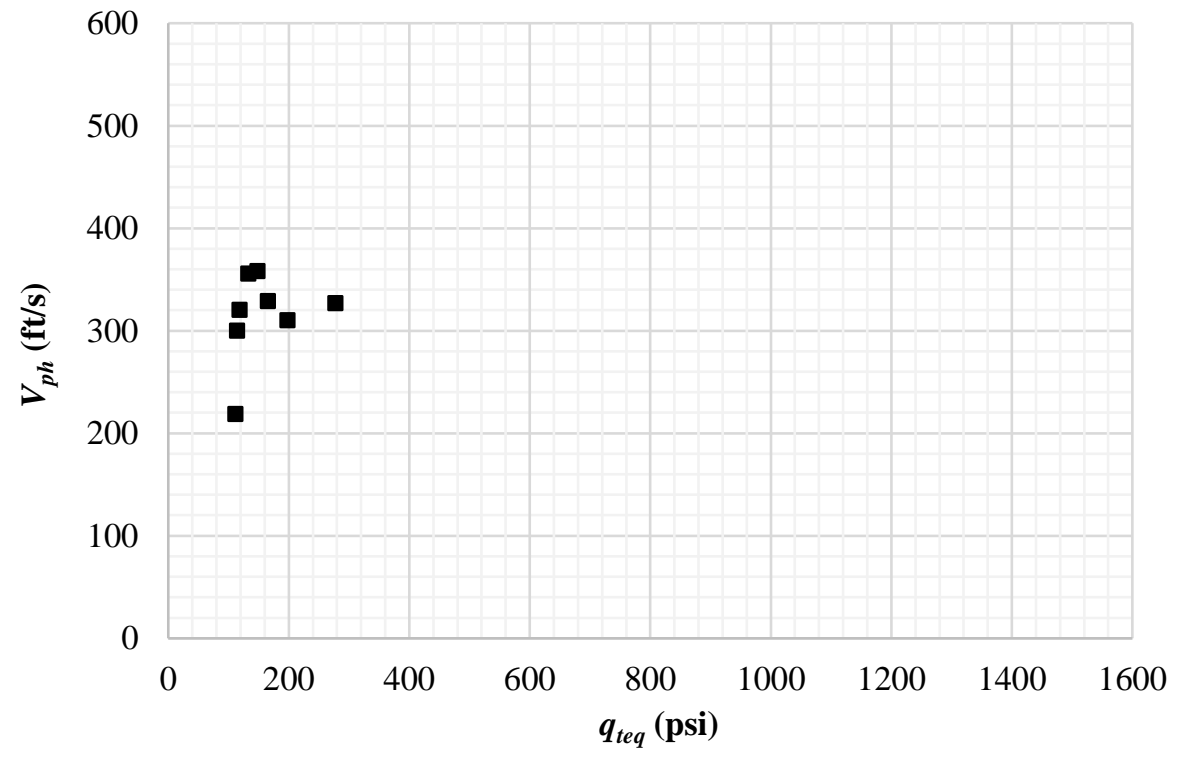

Figure A.32 - Pont 80 equivalent tip resistance $\left(q_{\text {teq }}\right)$ vs phase velocity $\left(V_{p h}\right)$ for the same $\lambda$. 


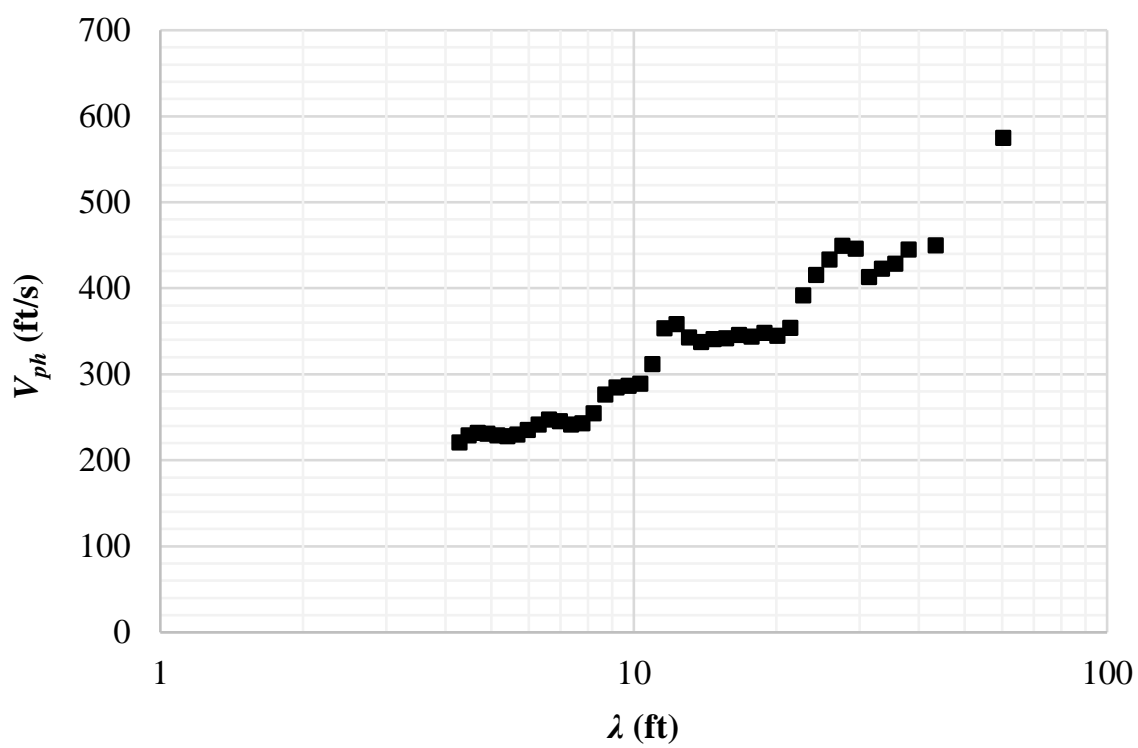

Figure A.33 - Point 94 dispersion curve.

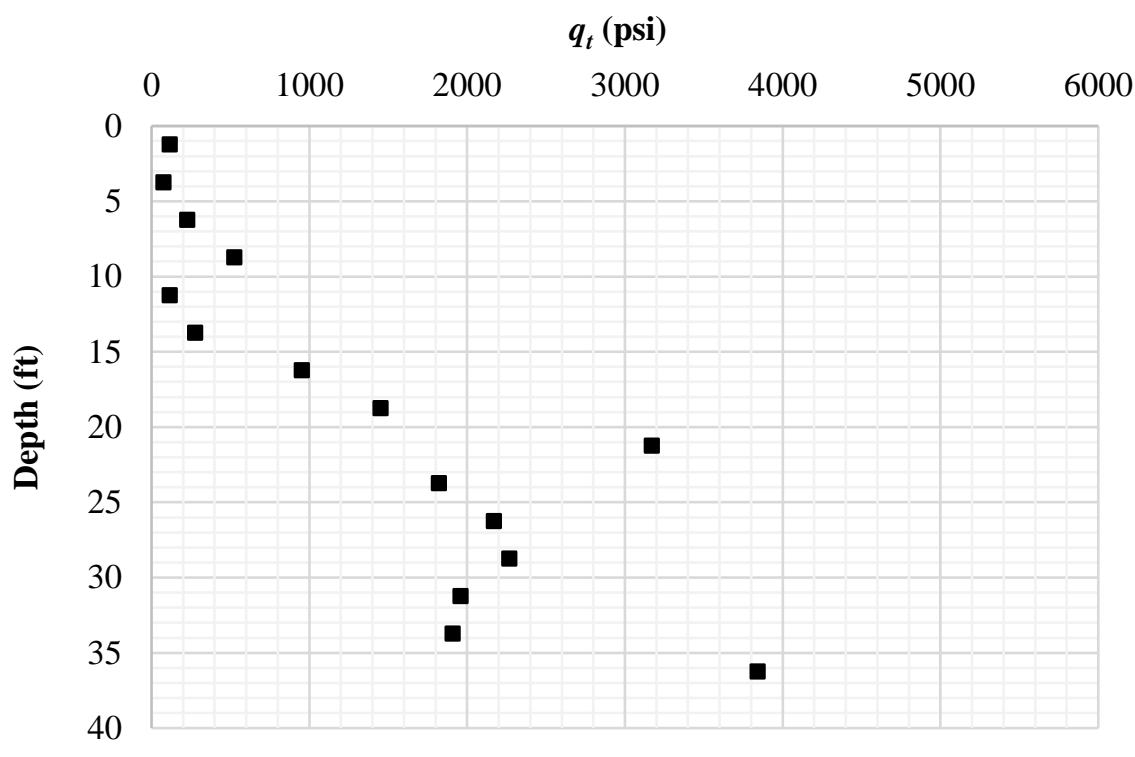

Figure A.34 - Point 94 tip resistance $\left(q_{t}\right)$ vs depth (z). 


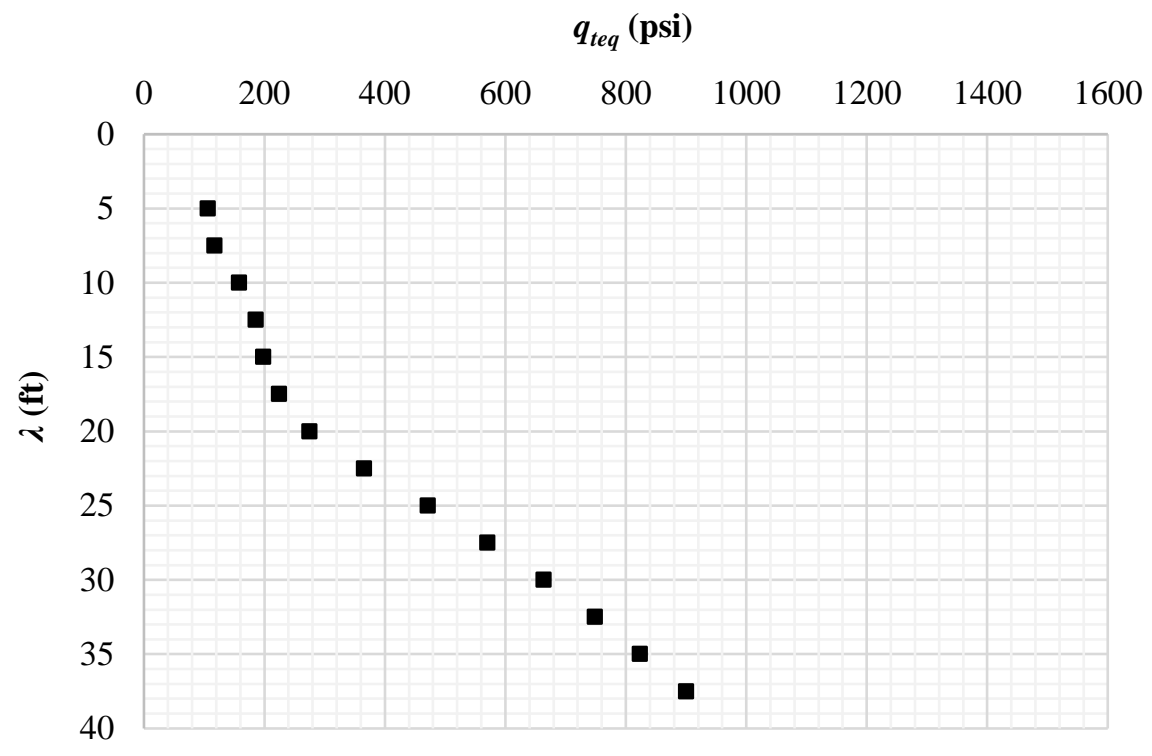

Figure A.35 - Point 94 equivalent tip resistance ( $\left.q_{t e q}\right)$ vs wavelength (ג).

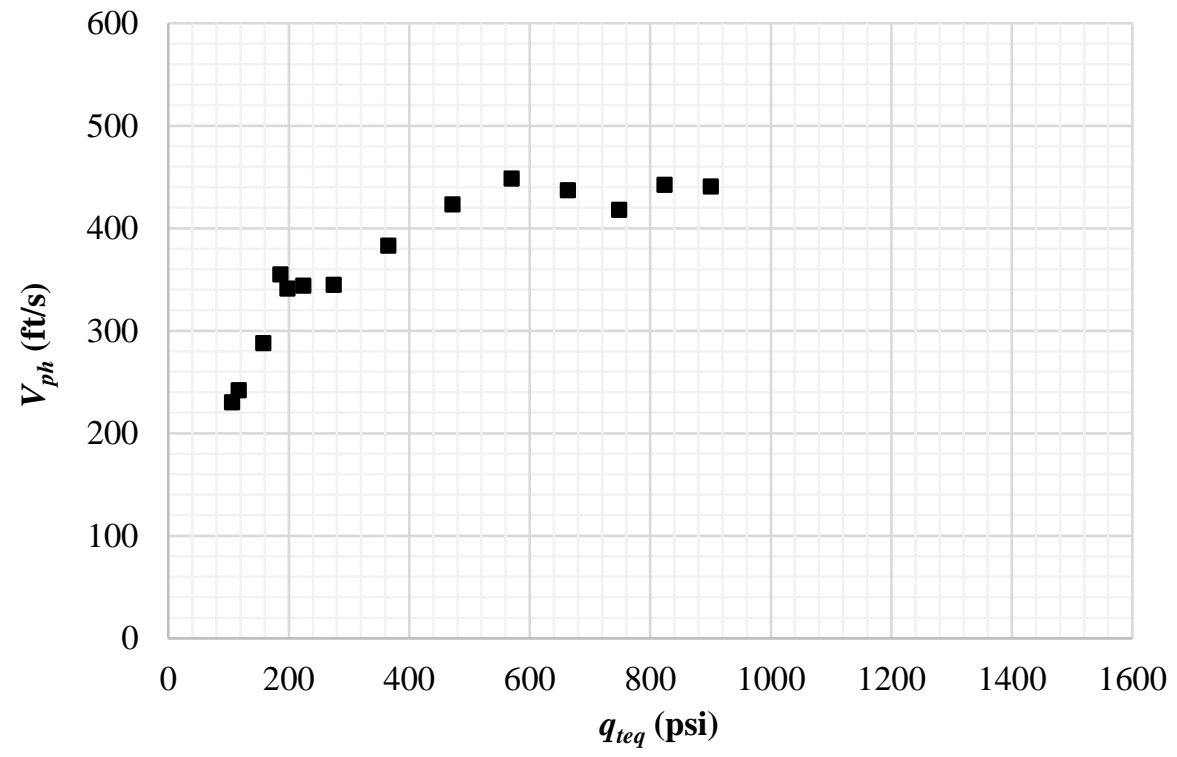

Figure A.36 - Pont 94 equivalent tip resistance $\left(q_{\text {teq }}\right)$ vs phase velocity $\left(V_{p h}\right)$ for the same $\lambda$. 


\section{Group $84 q_{t}$ vs depth}

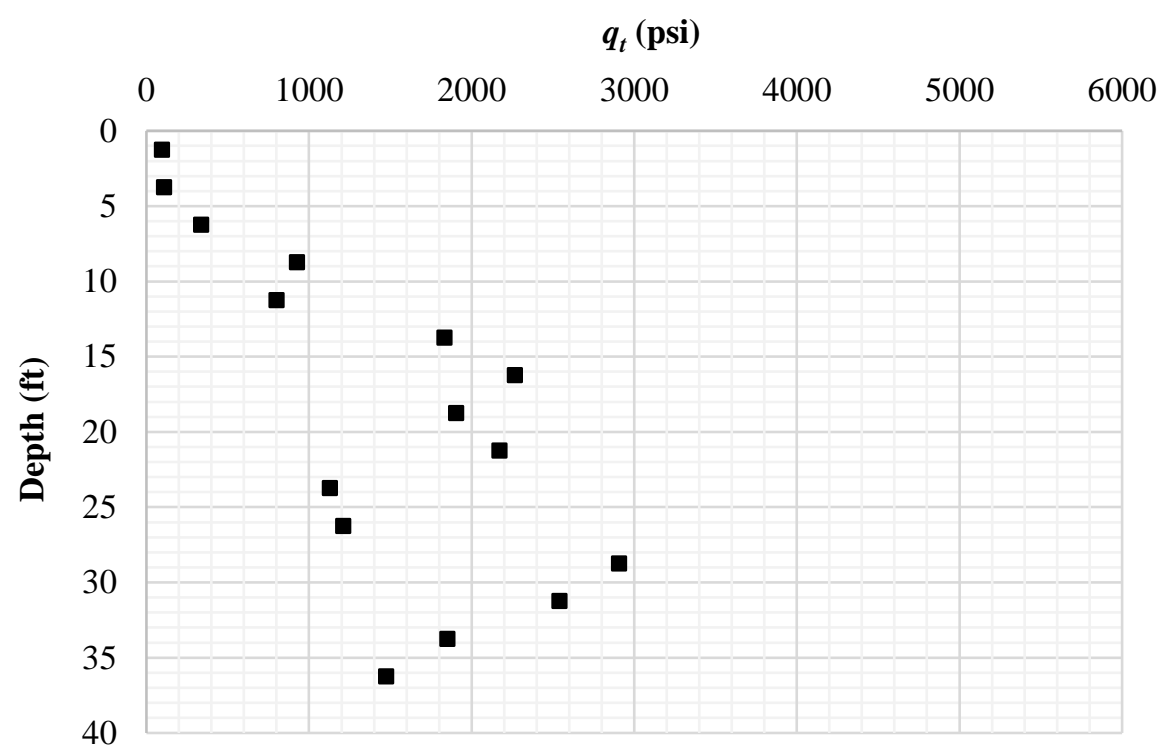

Figure A.37 - Point 68 tip resistance $\left(q_{t}\right)$ vs depth (z).

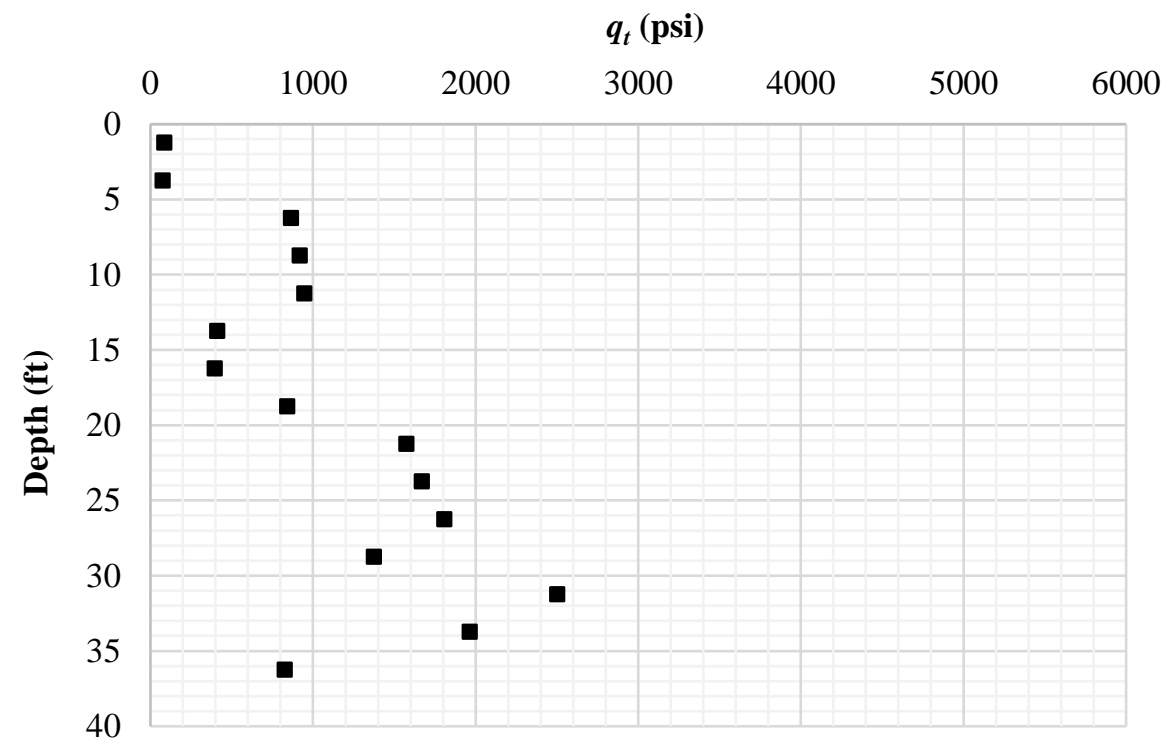

Figure A.38 - Point 70 tip resistance $\left(q_{t}\right)$ vs depth (z). 


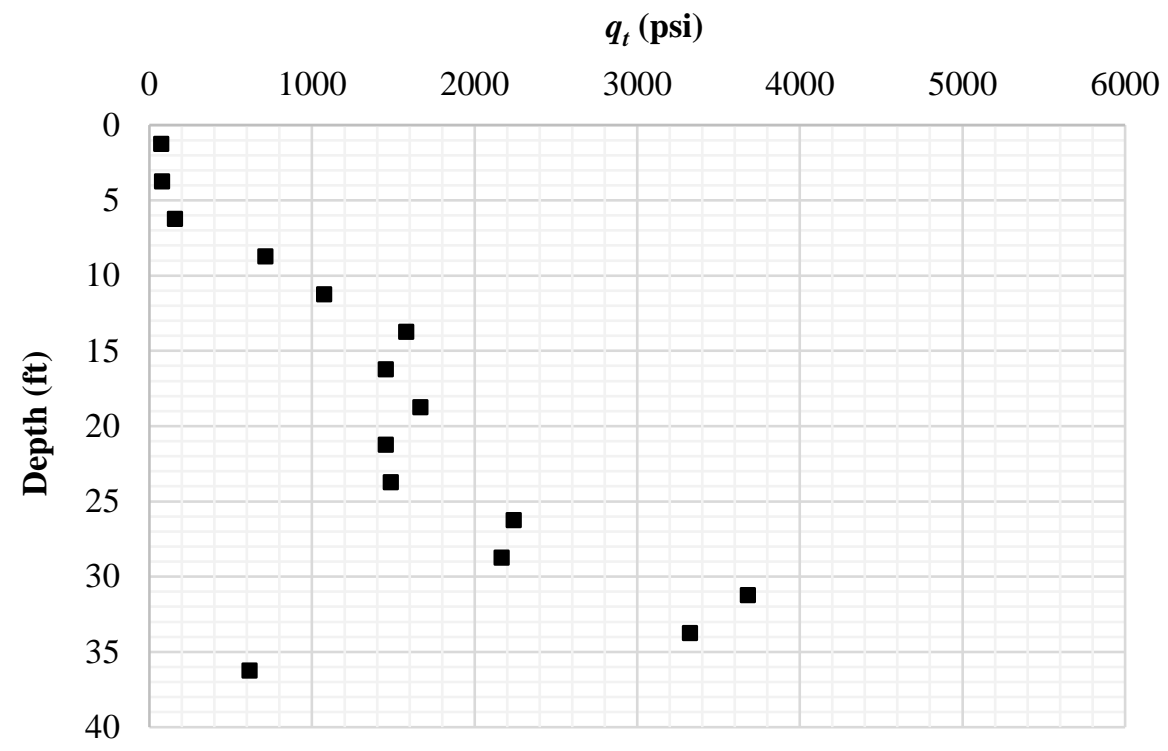

Figure A.39 - Point 84 tip resistance $\left(q_{t}\right)$ vs depth (z).

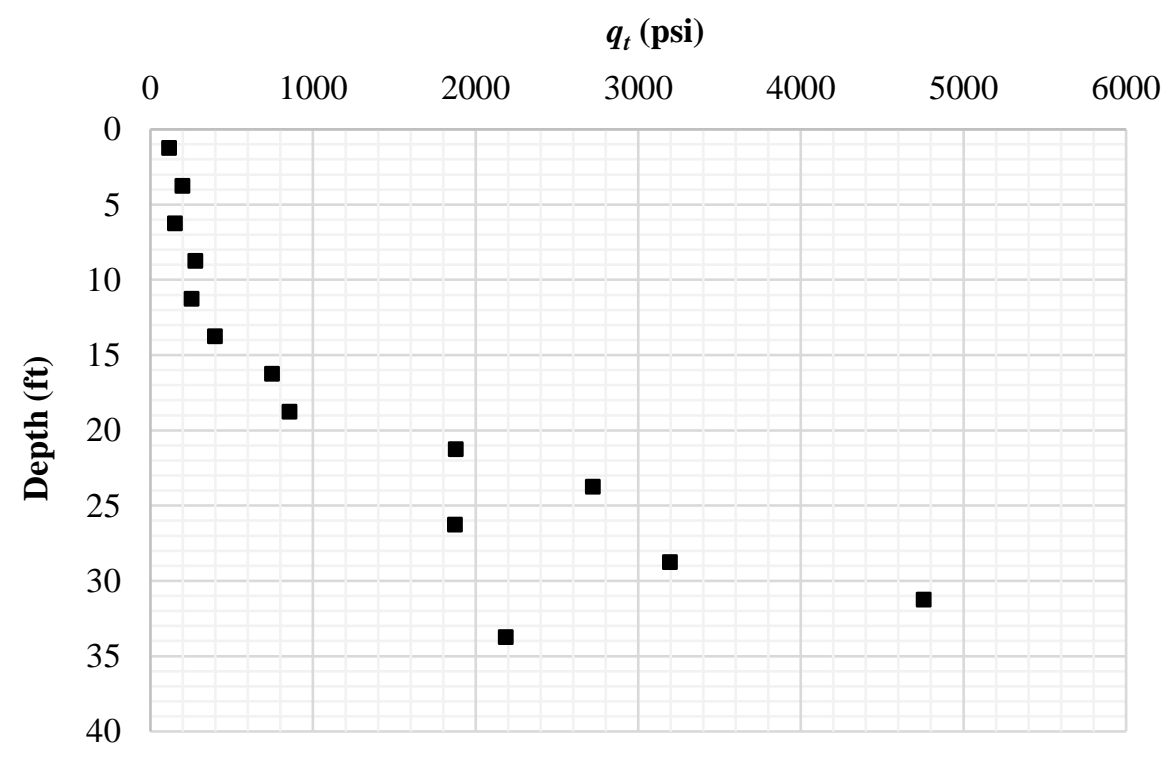

Figure A.40 - Point 96 tip resistance $\left(q_{t}\right)$ vs depth $(z)$. 


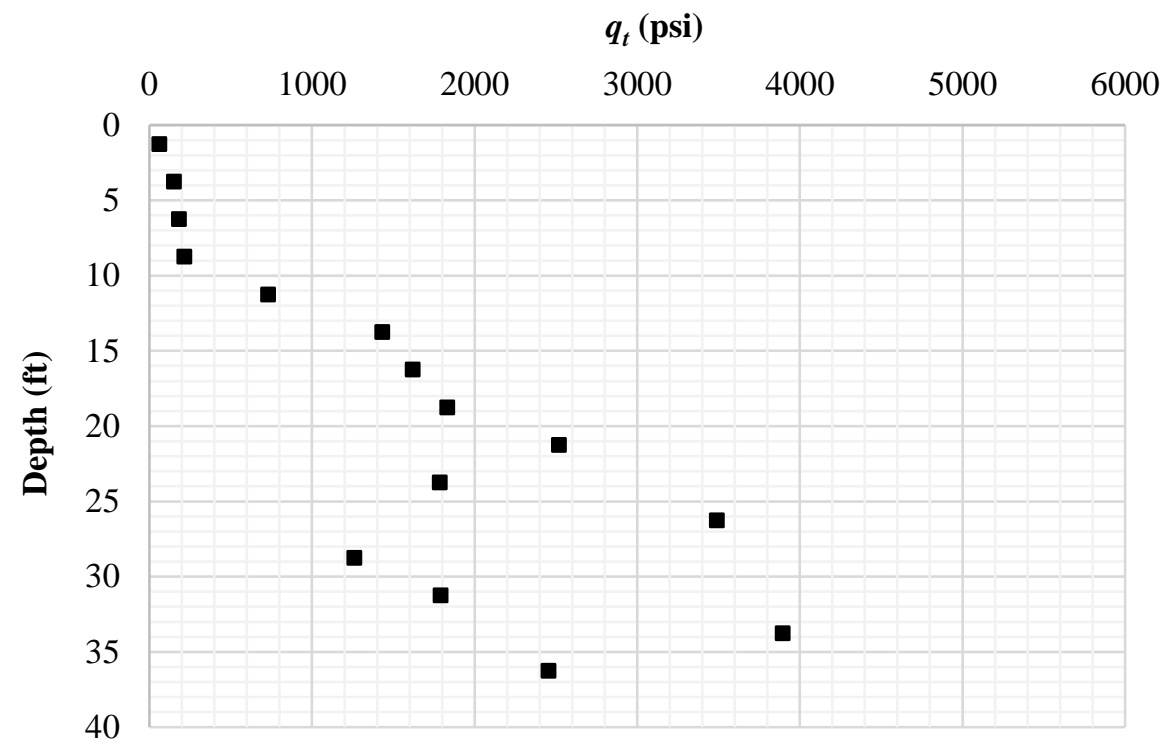

Figure A.41 - Point 98 tip resistance $\left(q_{t}\right)$ vs depth $(z)$. 


\section{Group 135}

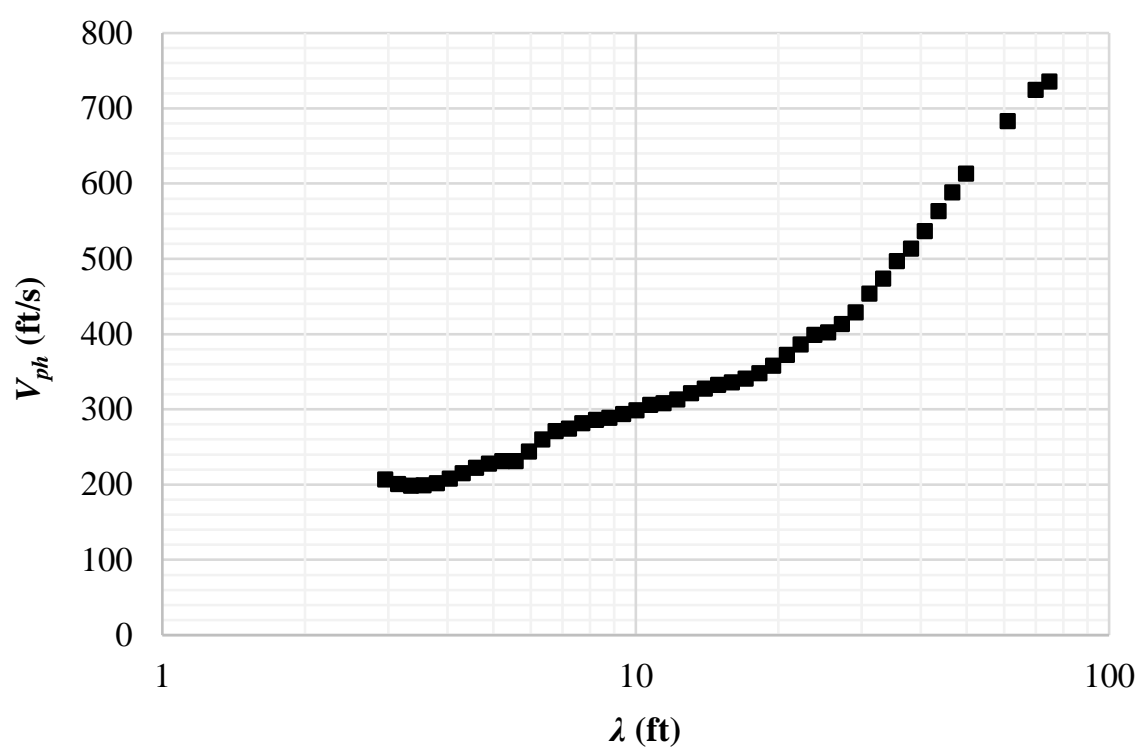

Figure A.42 - Point 121 dispersion curve.

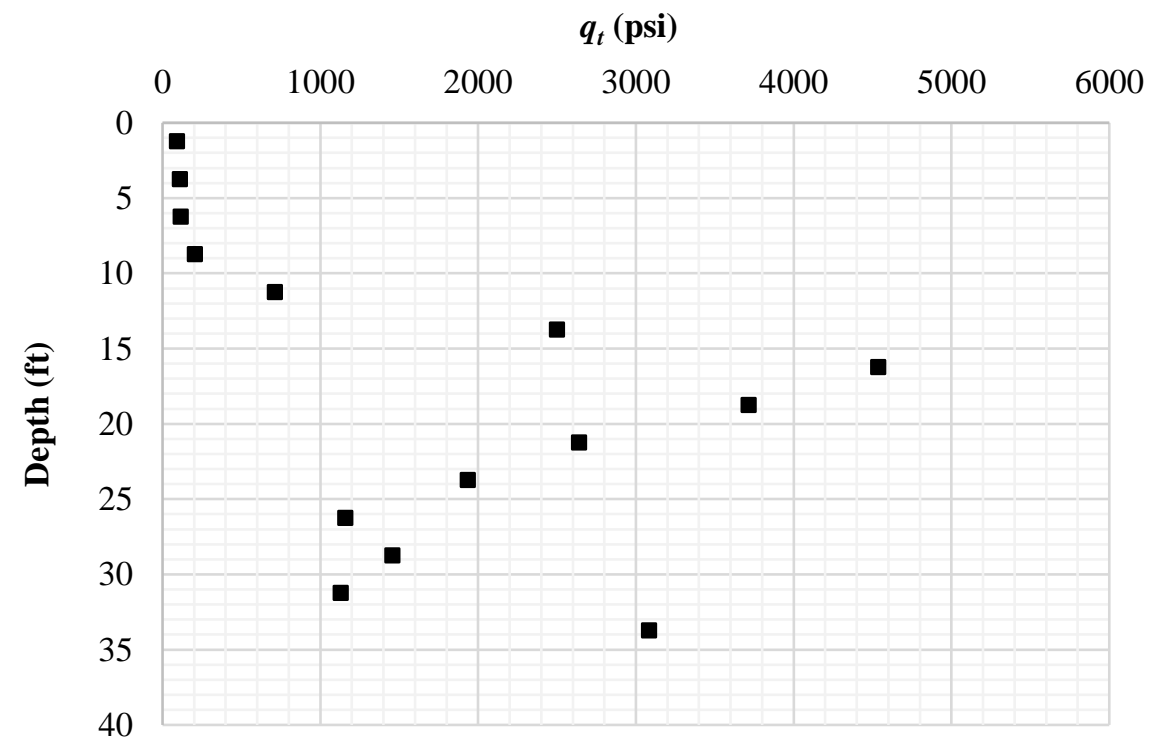

Figure A.43 - Point 121 tip resistance $\left(q_{t}\right)$ vs depth (z). 


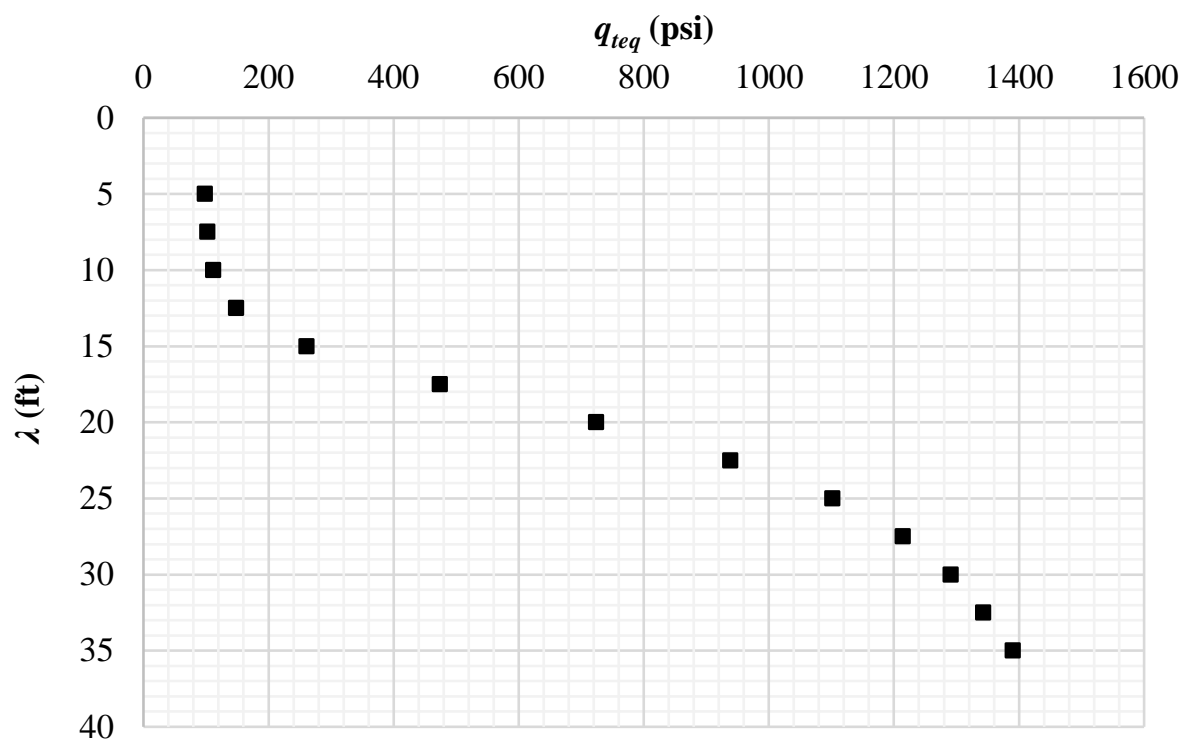

Figure A.44 - Point 121 equivalent tip resistance $\left(q_{t e q}\right)$ vs wavelength (ג).

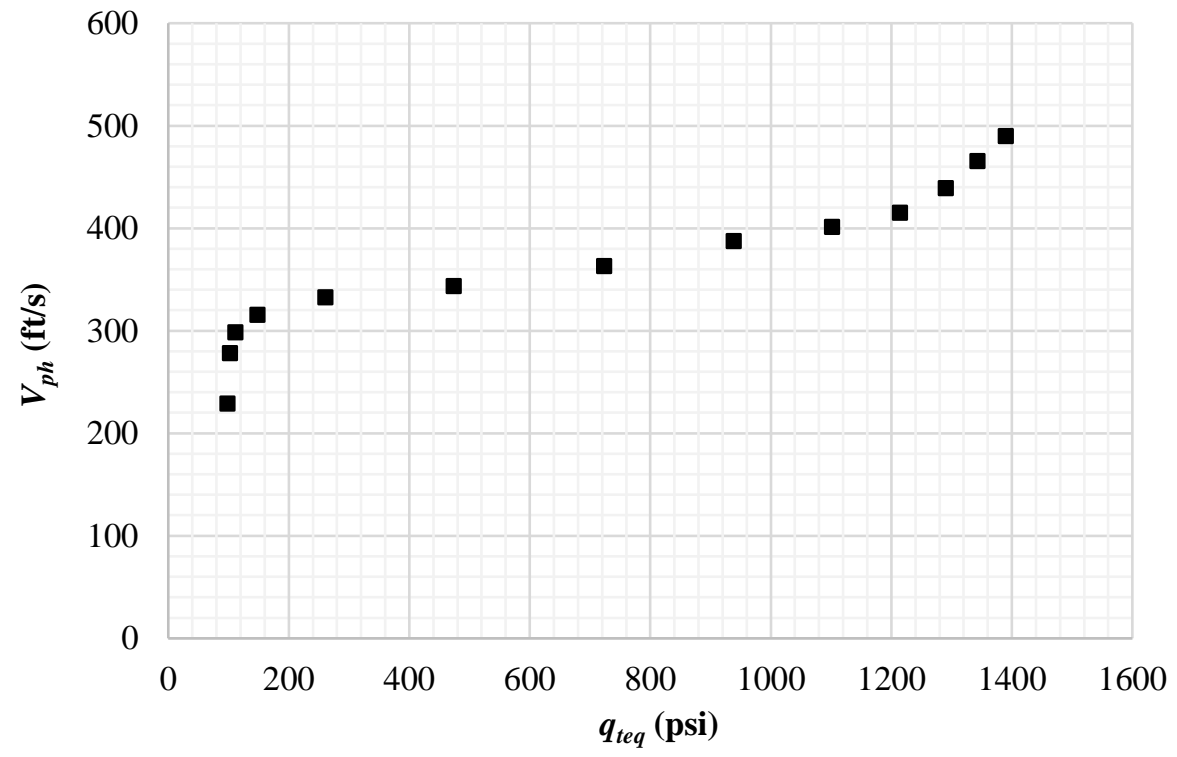

Figure A.45 - Pont 121 equivalent tip resistance $\left(q_{t e q}\right)$ vs phase velocity $\left(V_{p h}\right)$ for the same $\lambda$. 


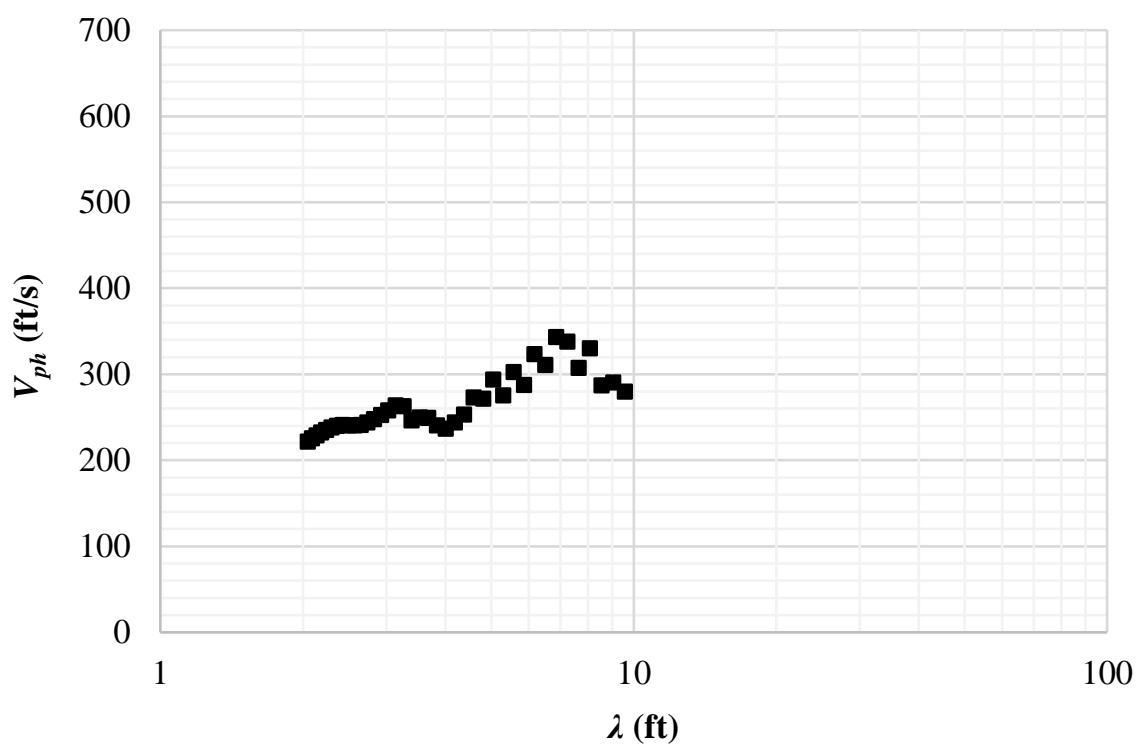

Figure A.46 - Point 123 dispersion curve.

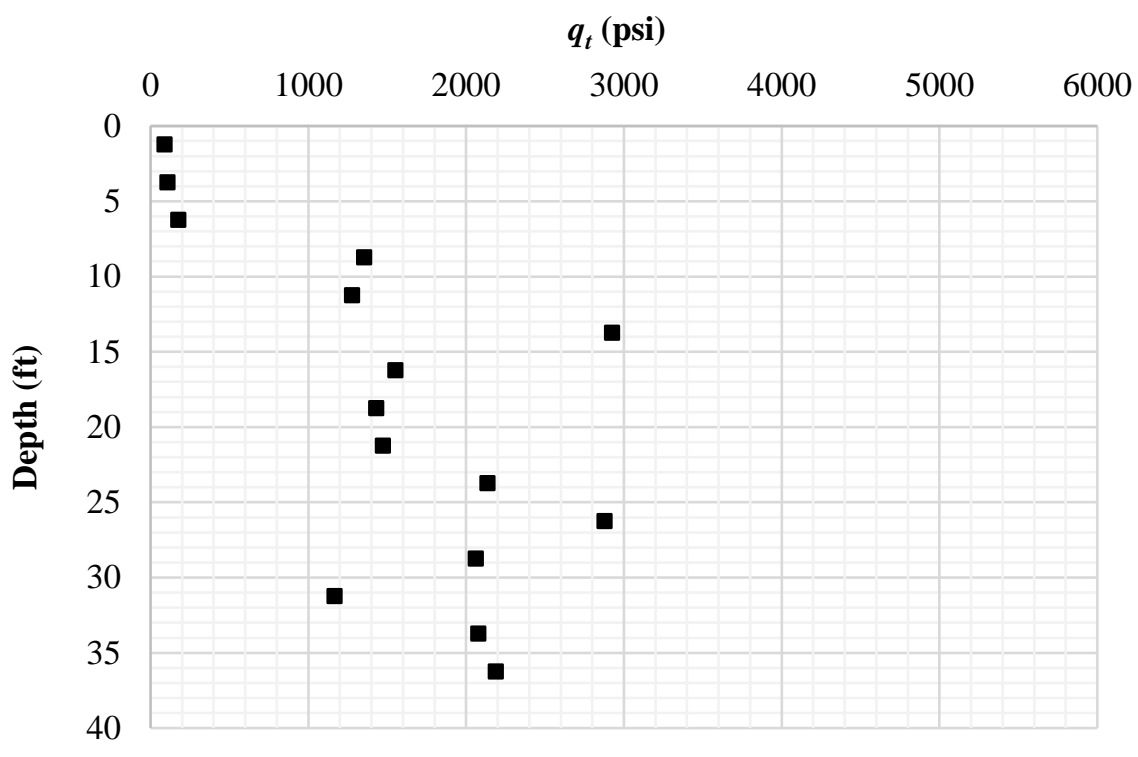

Figure A.47 - Point 123 tip resistance $\left(q_{t}\right)$ vs depth (z). 


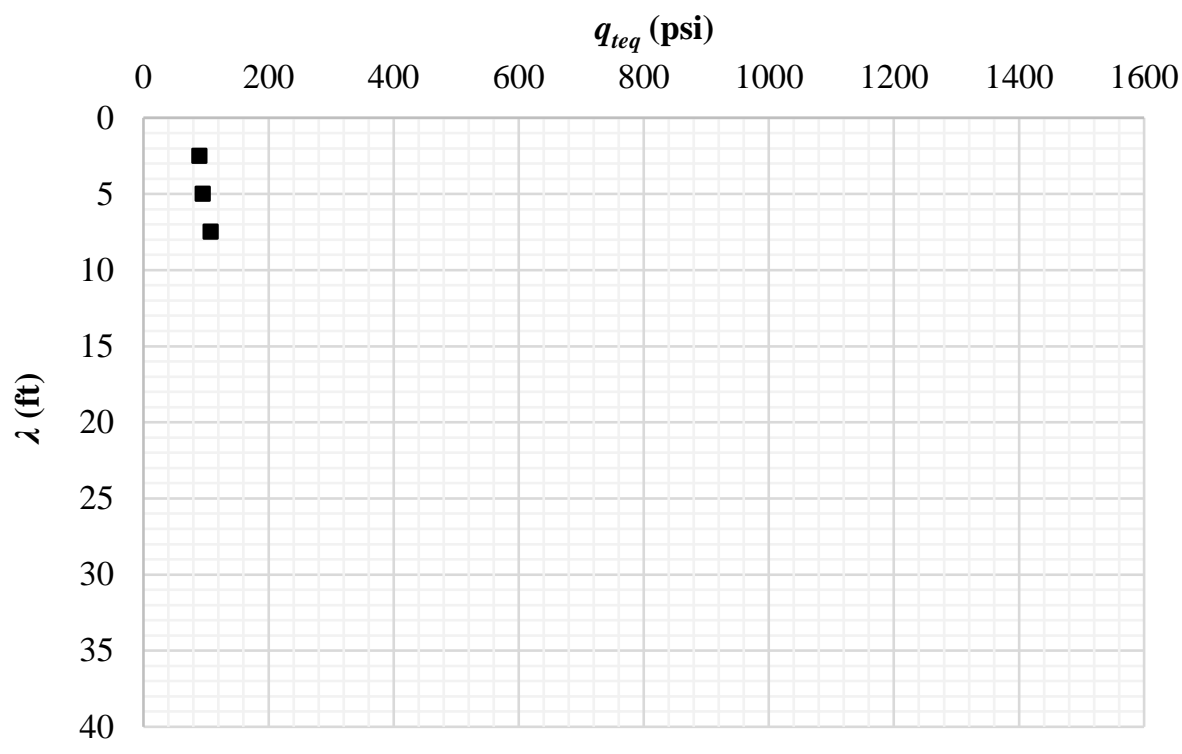

Figure A.48 - Point 123 equivalent tip resistance $\left(q_{t e q}\right)$ vs wavelength (ג).

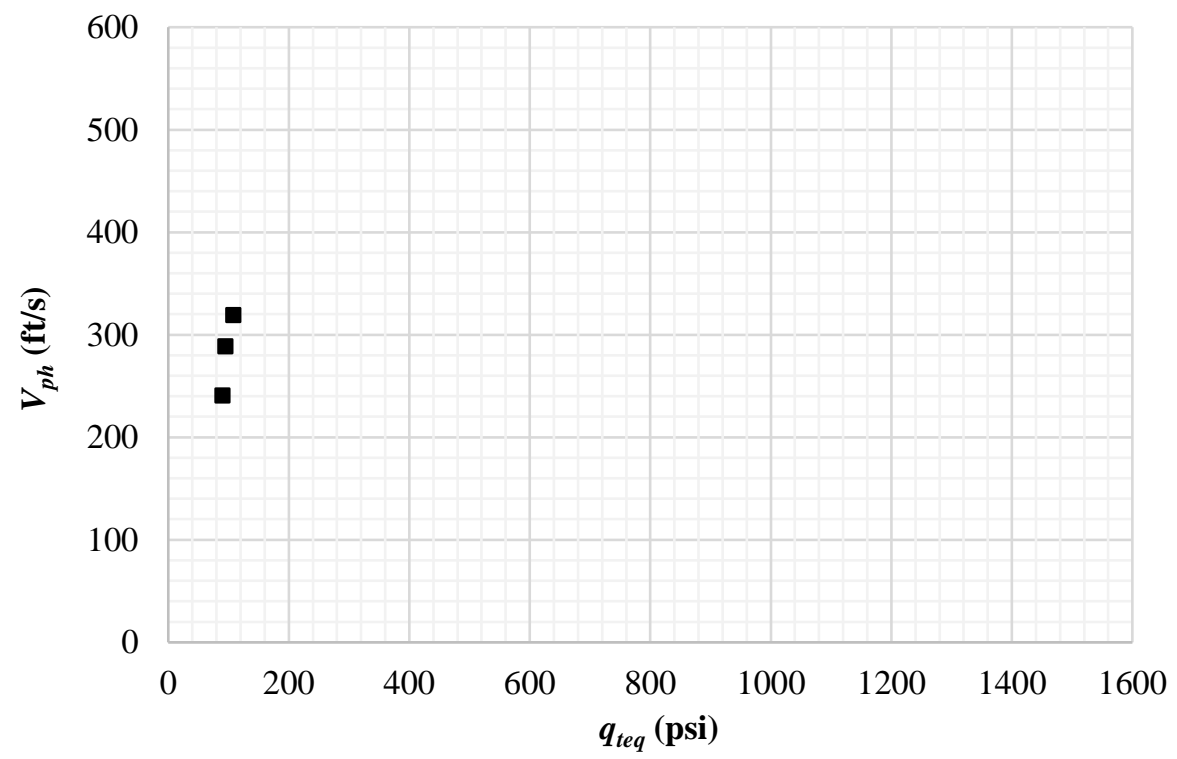

Figure A.49 - Pont 123 equivalent tip resistance $\left(q_{t e q}\right)$ vs phase velocity $\left(V_{p h}\right)$ for the same $\lambda$. 


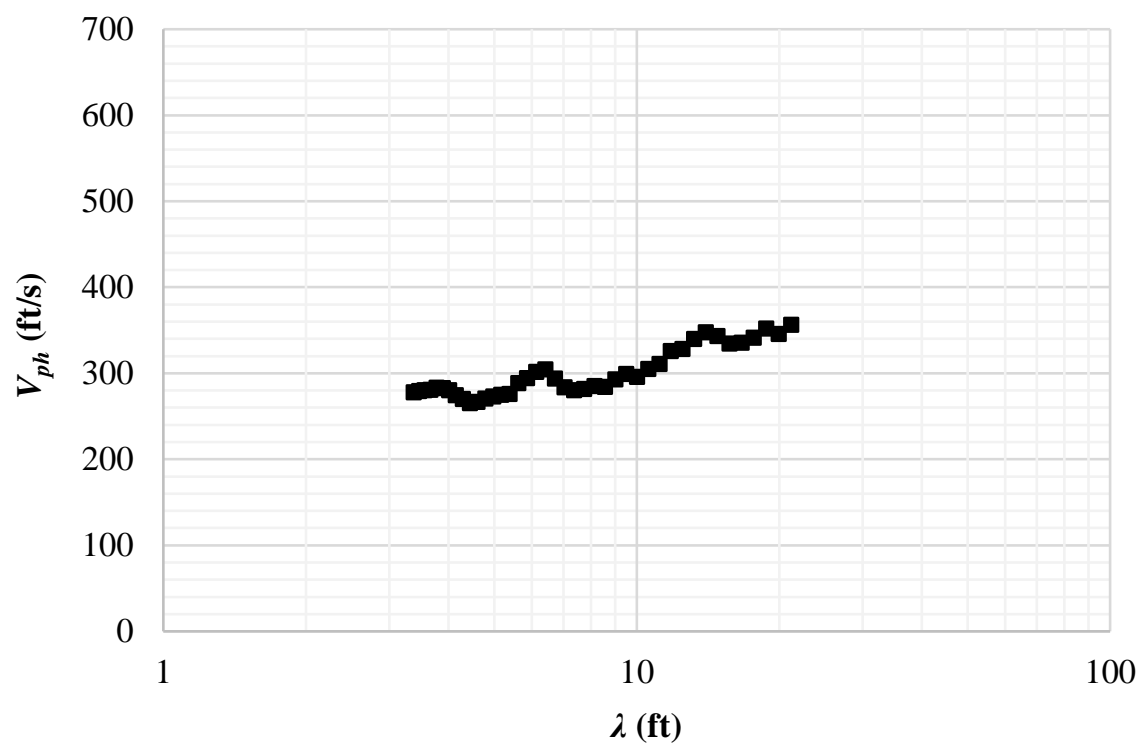

Figure A.50 - Point 135 dispersion curve.

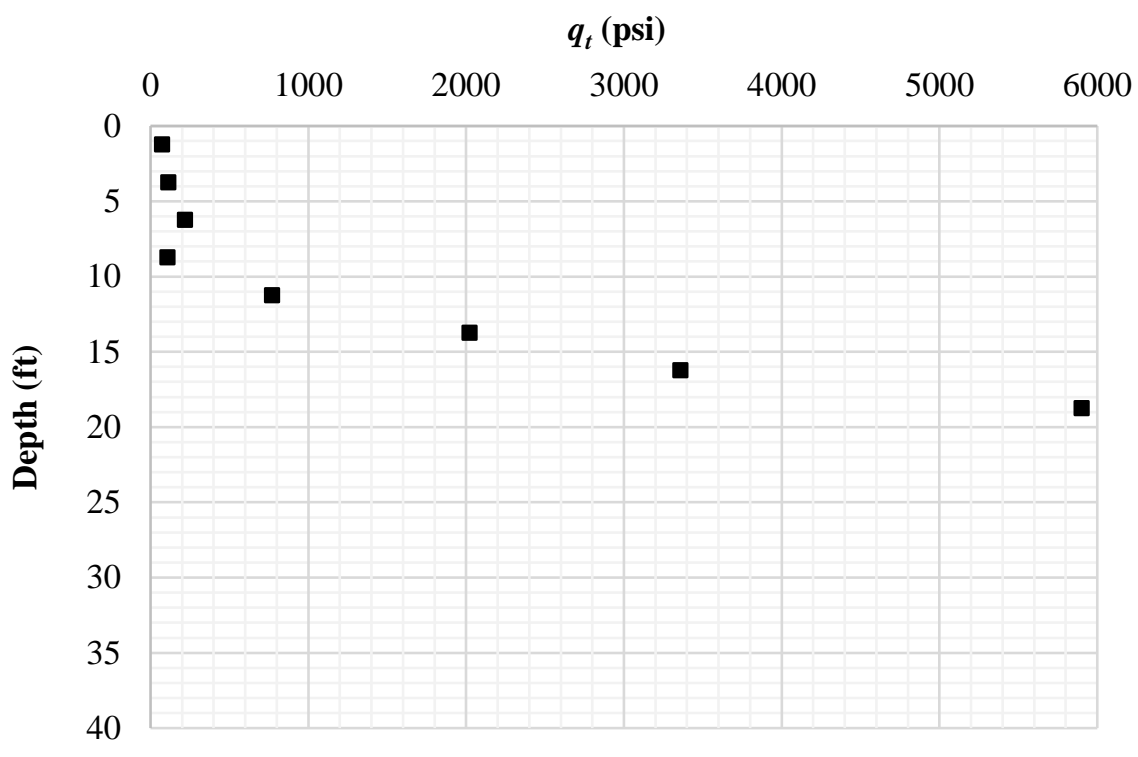

Figure A.51 - Point 135 tip resistance $\left(q_{t}\right)$ vs depth (z). 


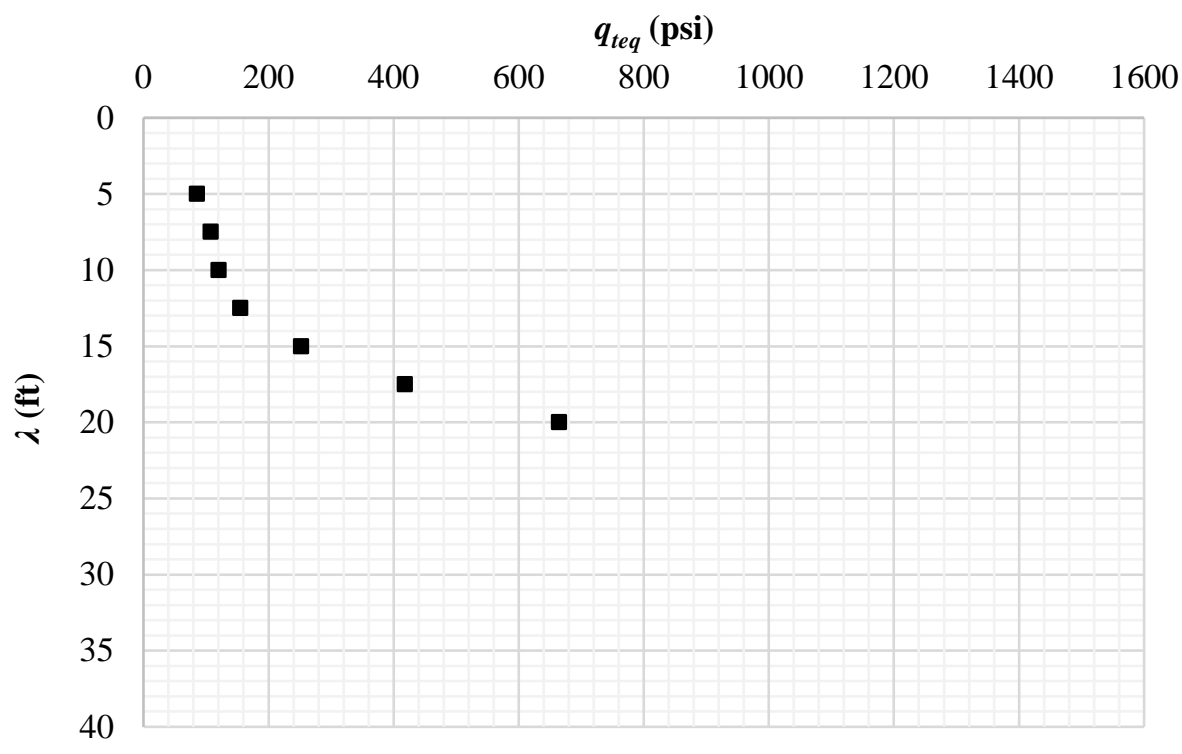

Figure A.52 - Point 135 equivalent tip resistance $\left(q_{t e q}\right)$ vs wavelength (ג).

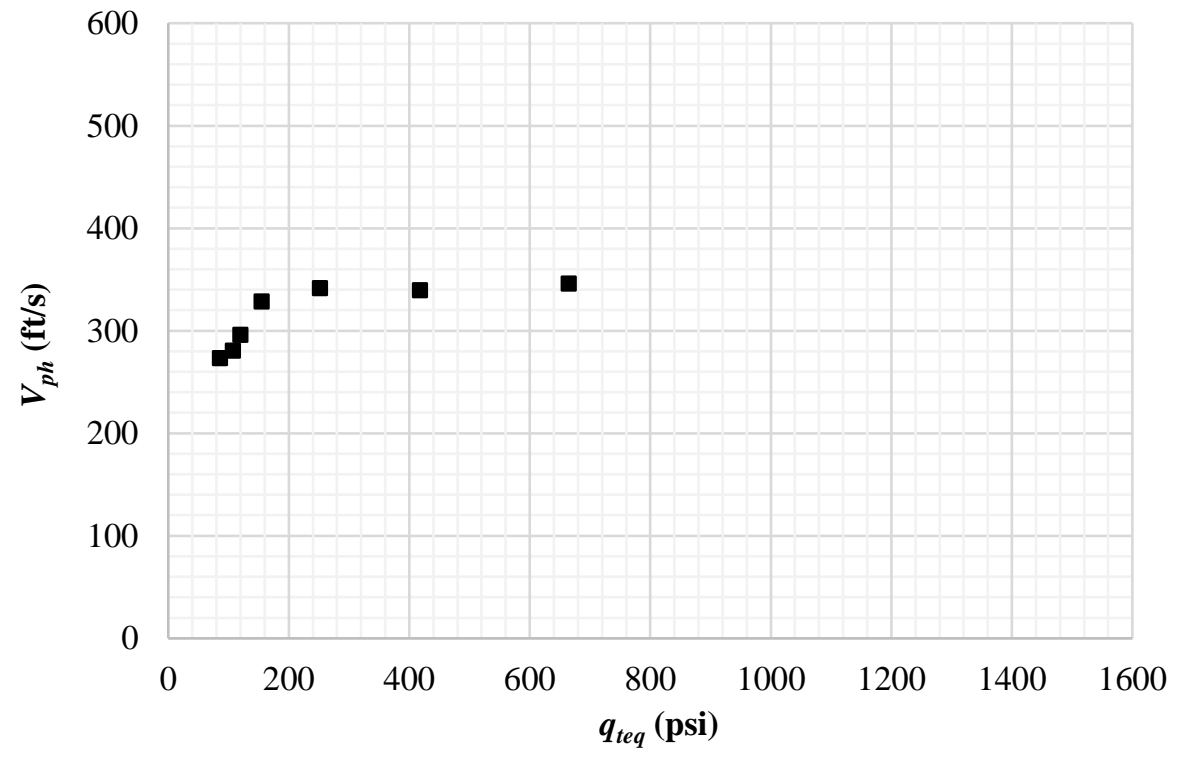

Figure A.53 - Pont 135 equivalent tip resistance $\left(q_{\text {teq }}\right)$ vs phase velocity $\left(V_{p h}\right)$ for the same $\lambda$. 


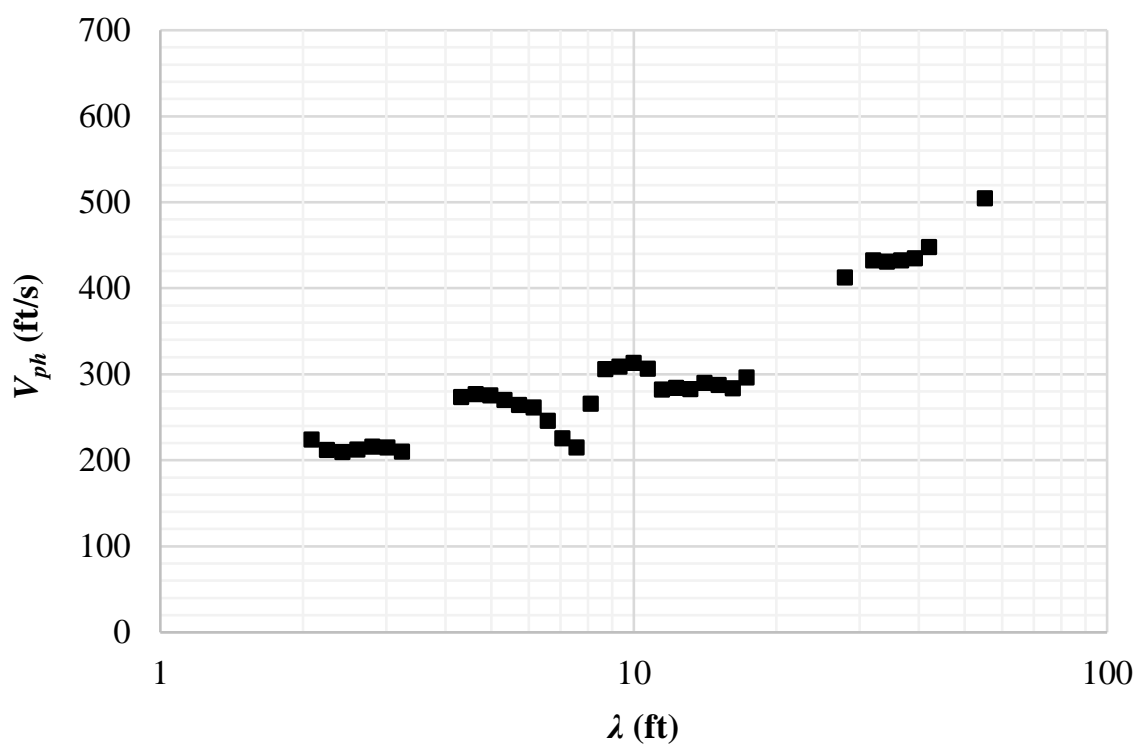

Figure A.54 - Point 147A dispersion curve.

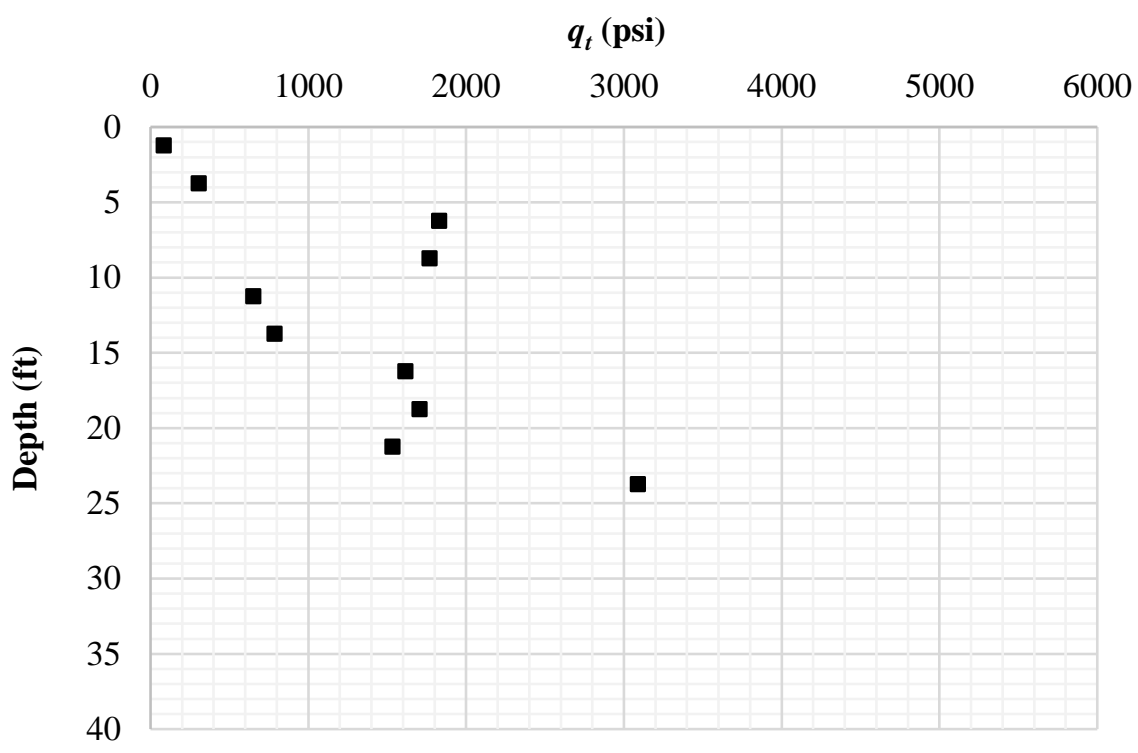

Figure A.55 - Point 147A tip resistance $\left(q_{t}\right)$ vs depth (z). 


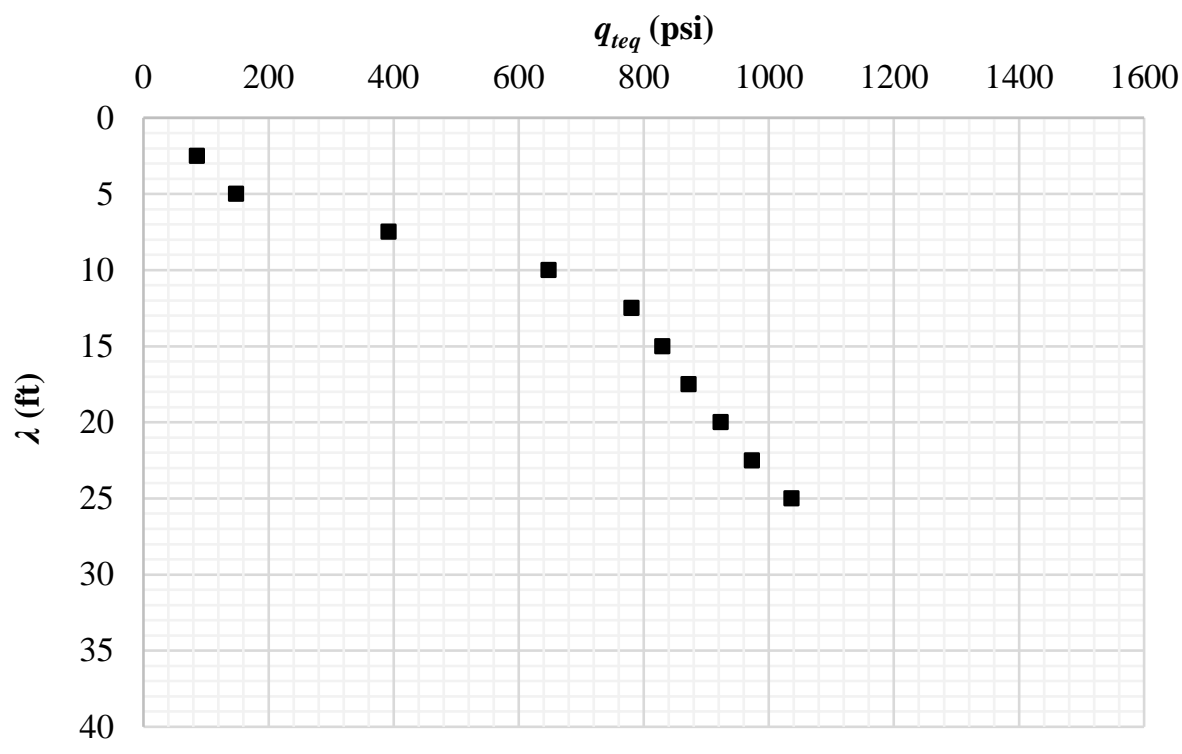

Figure A.56 - Point 147A equivalent tip resistance $\left(q_{t e q}\right)$ vs wavelength (ג).

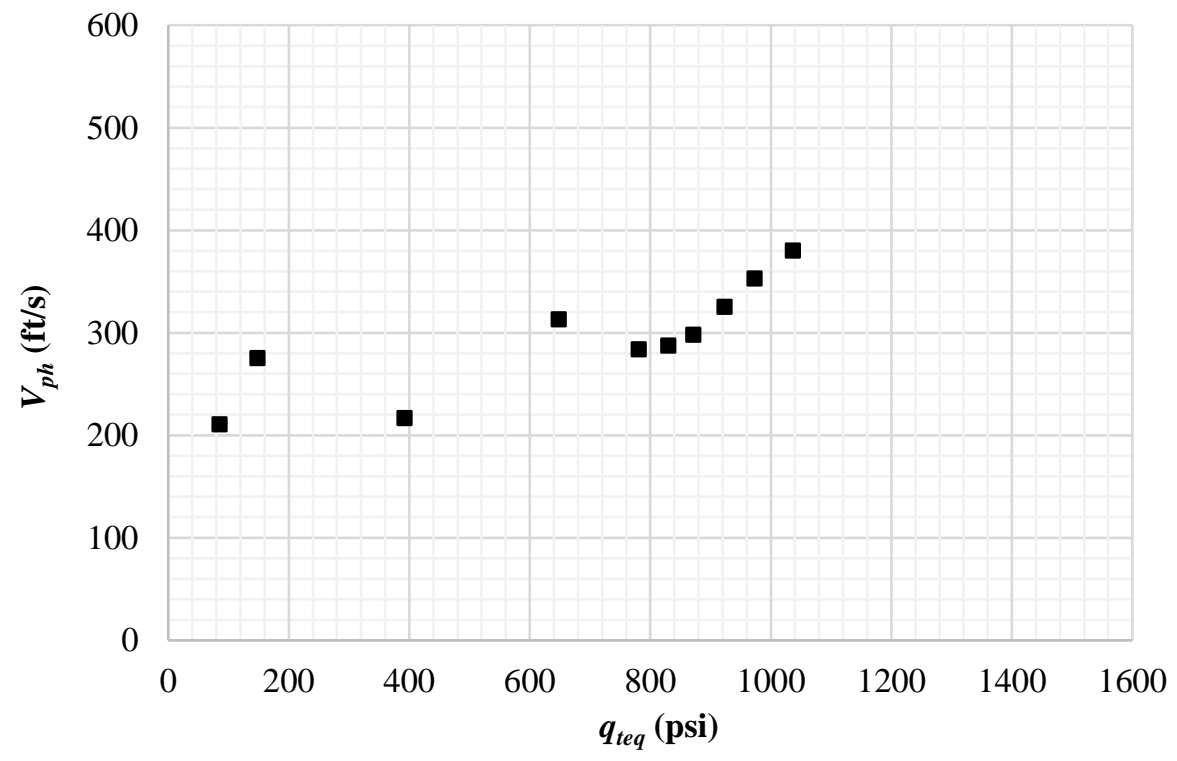

Figure A.57 - Pont 147A equivalent tip resistance $\left(q_{\text {teq }}\right)$ vs phase velocity $\left(V_{p h}\right)$ for the same $\lambda$. 


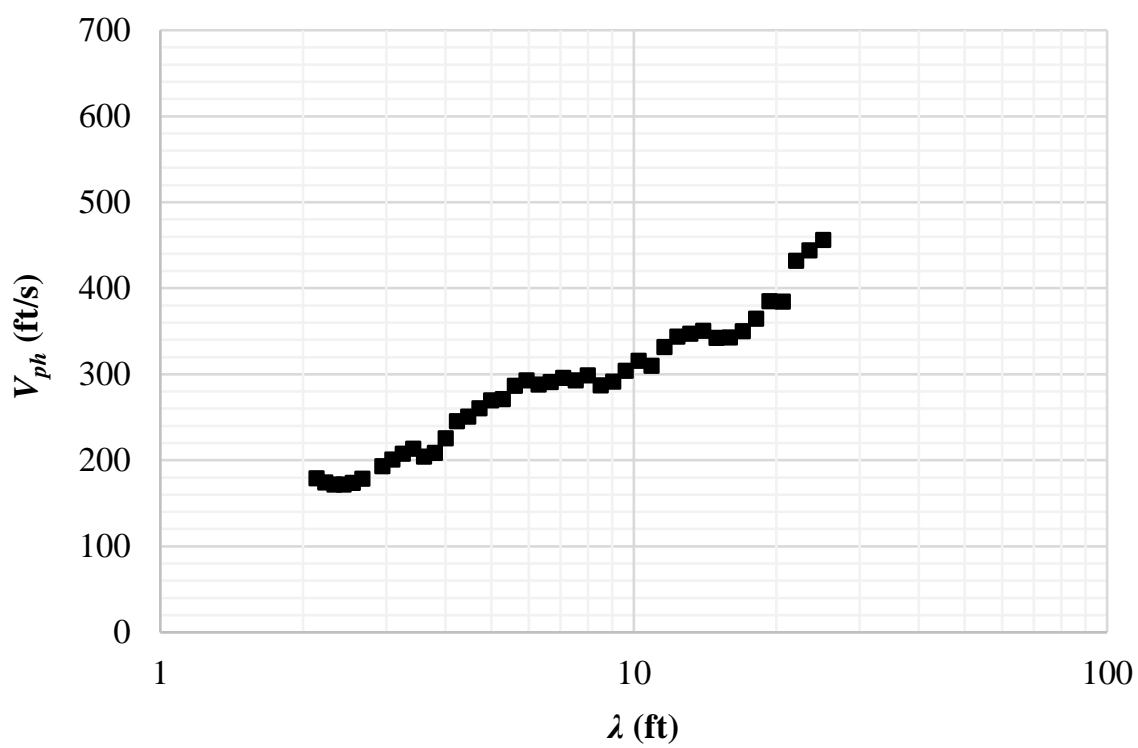

Figure A.58 - Point 149 dispersion curve.

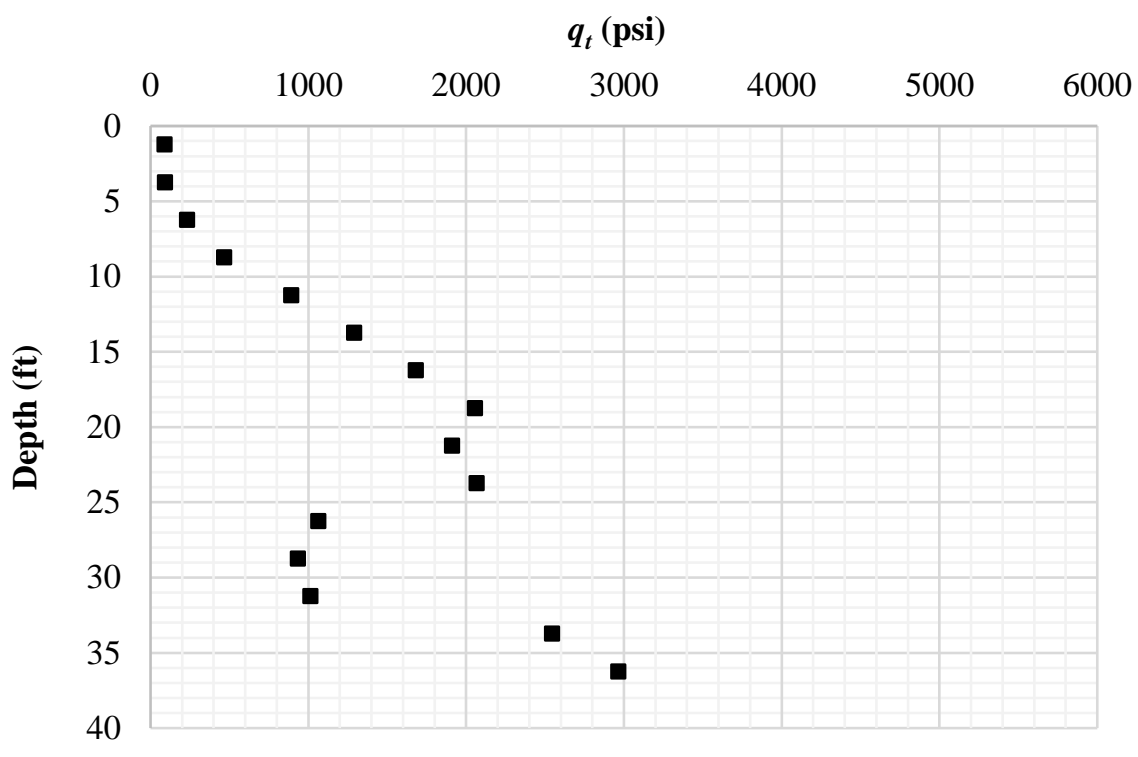

Figure A.59 - Point 149 tip resistance $\left(q_{t}\right)$ vs depth (z). 


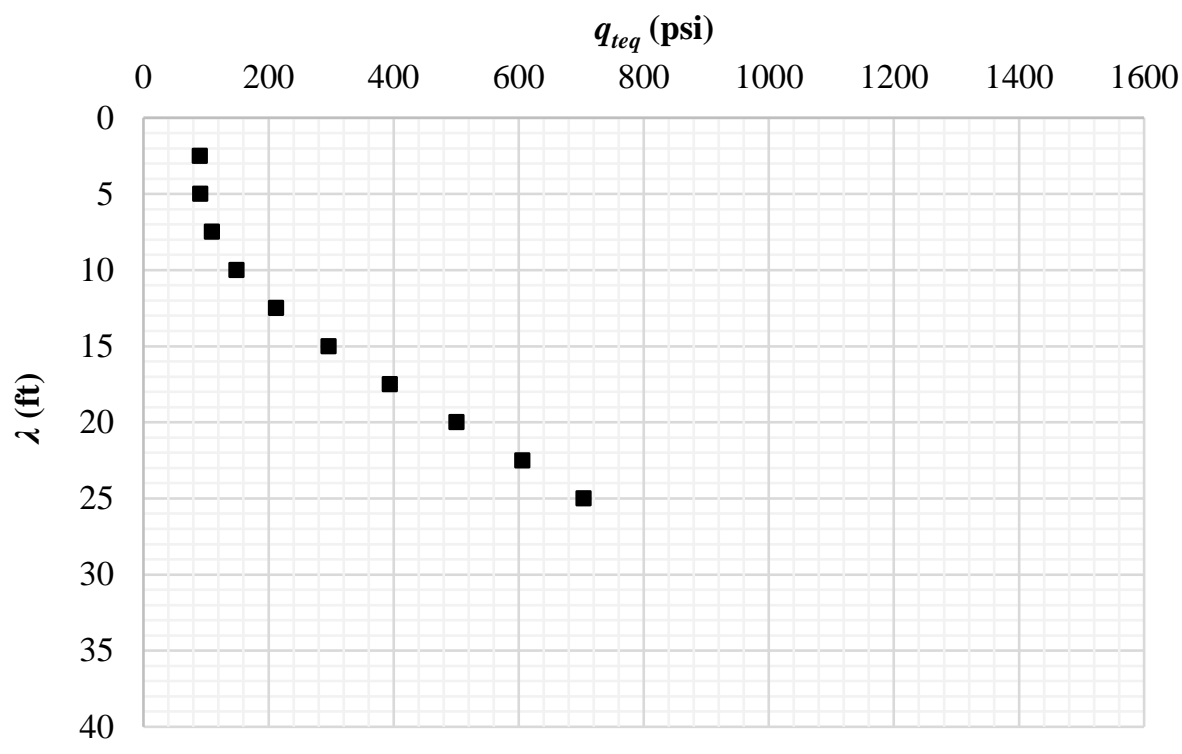

Figure A.60 - Point 149 equivalent tip resistance $\left(q_{\text {teq }}\right)$ vs wavelength (ג).

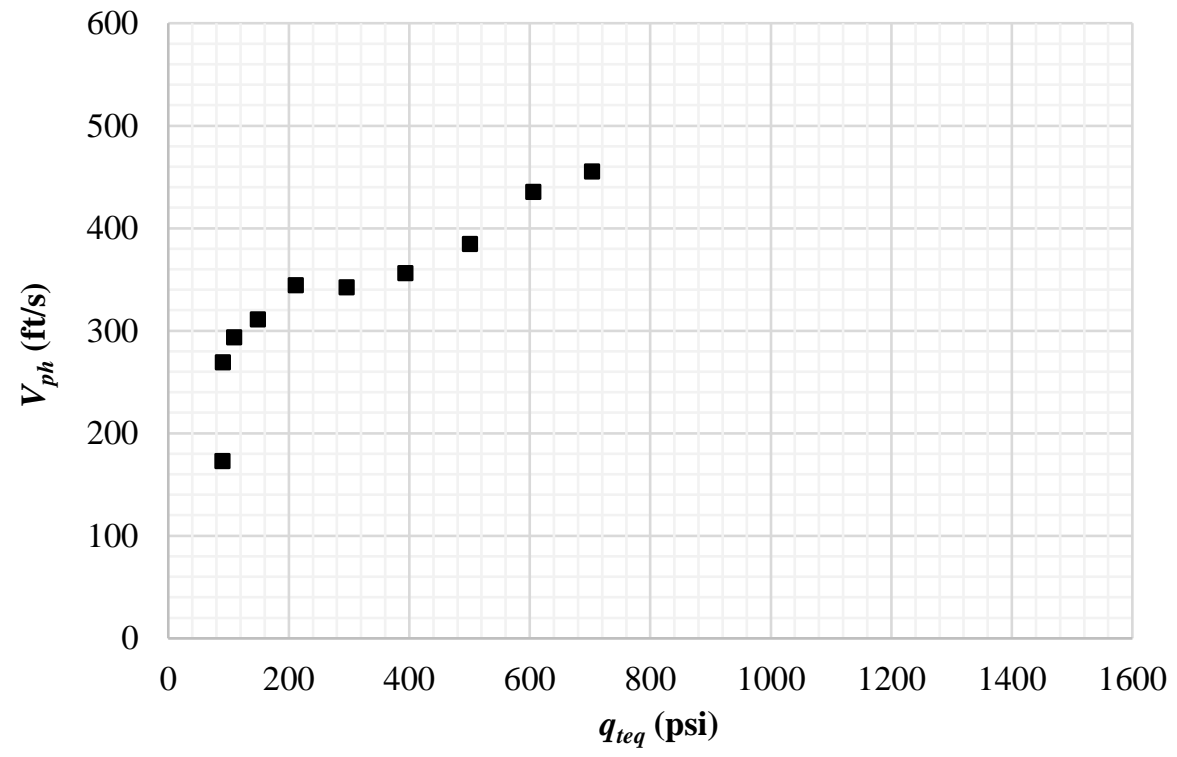

Figure A.61 - Pont 149 equivalent tip resistance $\left(q_{t e q}\right)$ vs phase velocity $\left(V_{p h}\right)$ for the same $\lambda$. 


\section{Group 180}

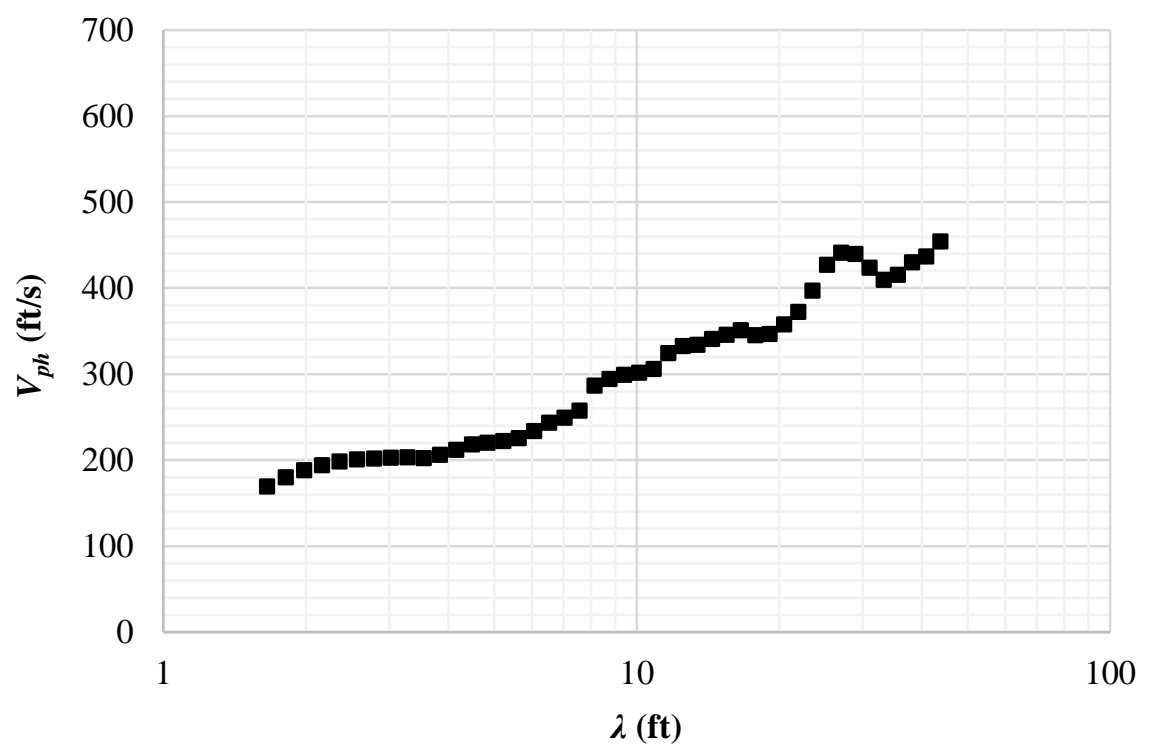

Figure A.62 - Point 168 dispersion curve.

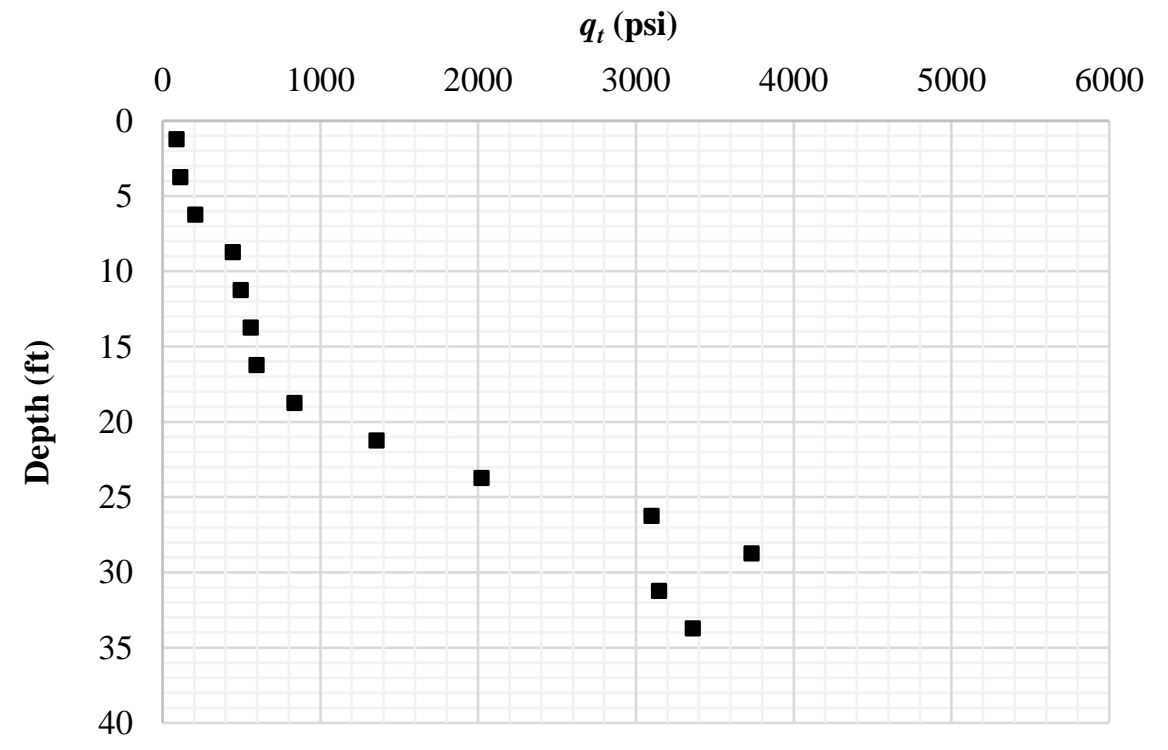

Figure A.63 - Point 168 tip resistance $\left(q_{t}\right)$ vs depth (z). 


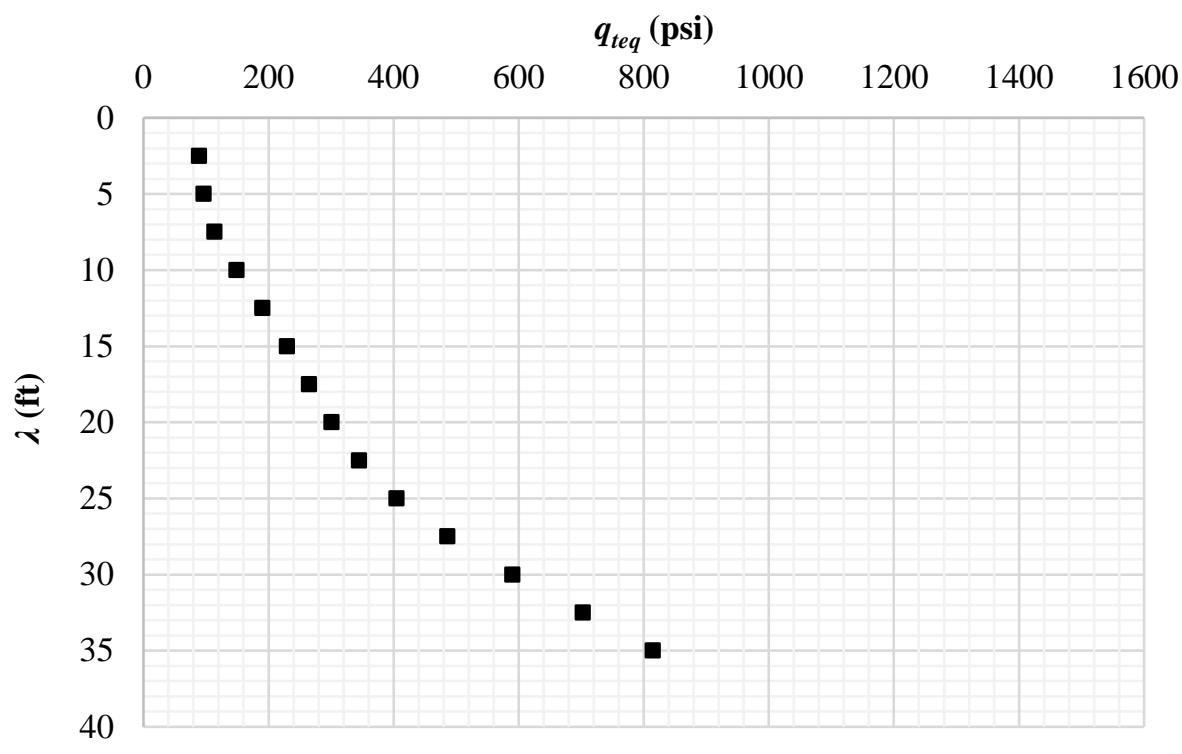

Figure A.64 - Point 168 equivalent tip resistance $\left(q_{\text {teq }}\right)$ vs wavelength (ג).

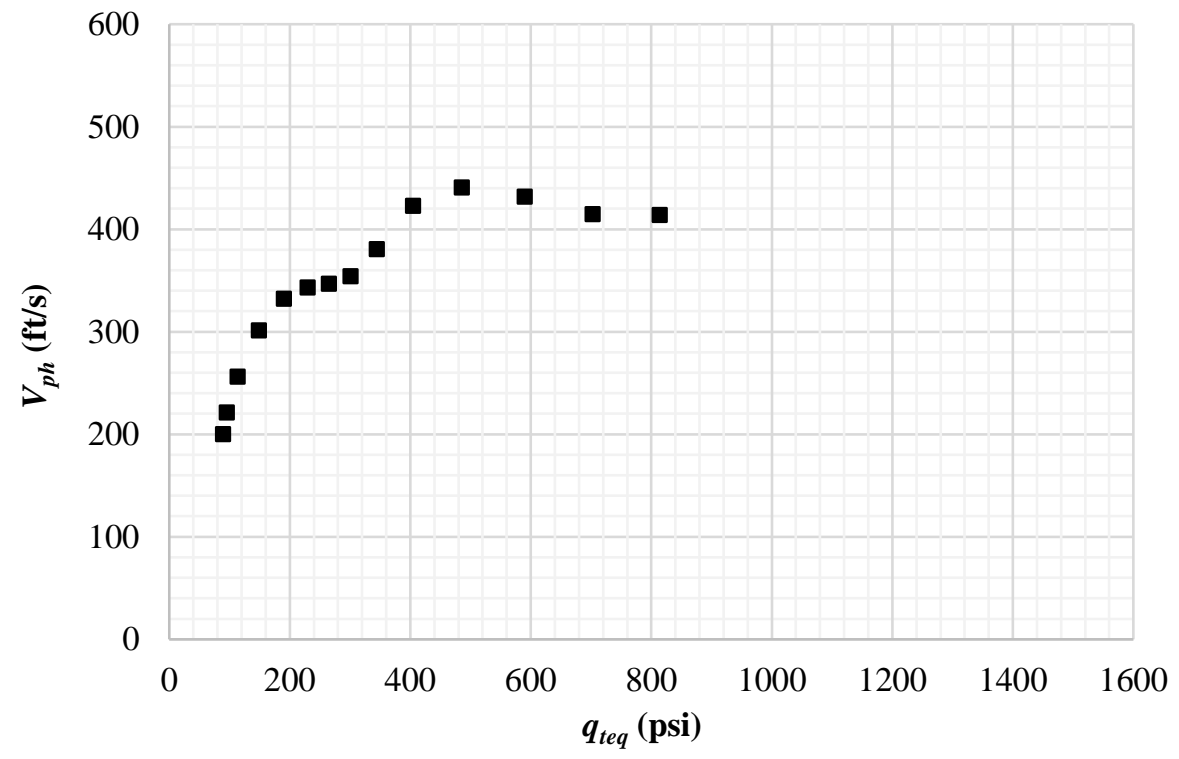

Figure A.65 - Pont 168 equivalent tip resistance $\left(q_{\text {teq }}\right)$ vs phase velocity $\left(V_{p h}\right)$ for the same $\lambda$. 


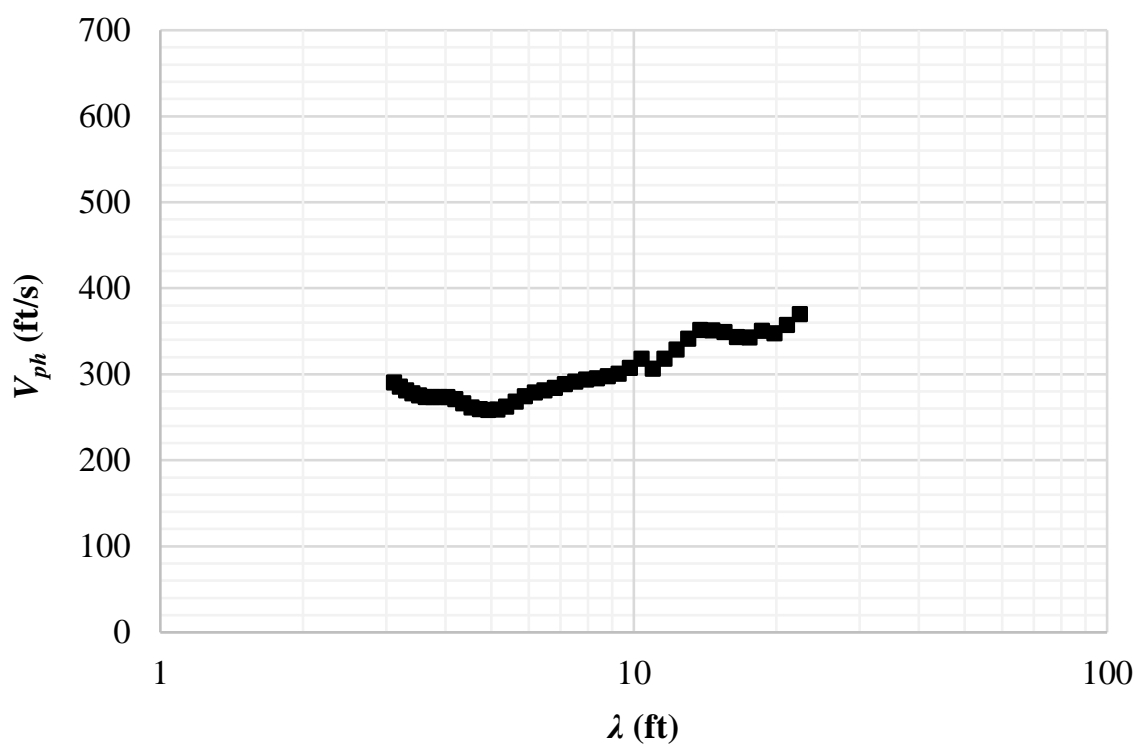

Figure A.66 - Point 170 dispersion curve.

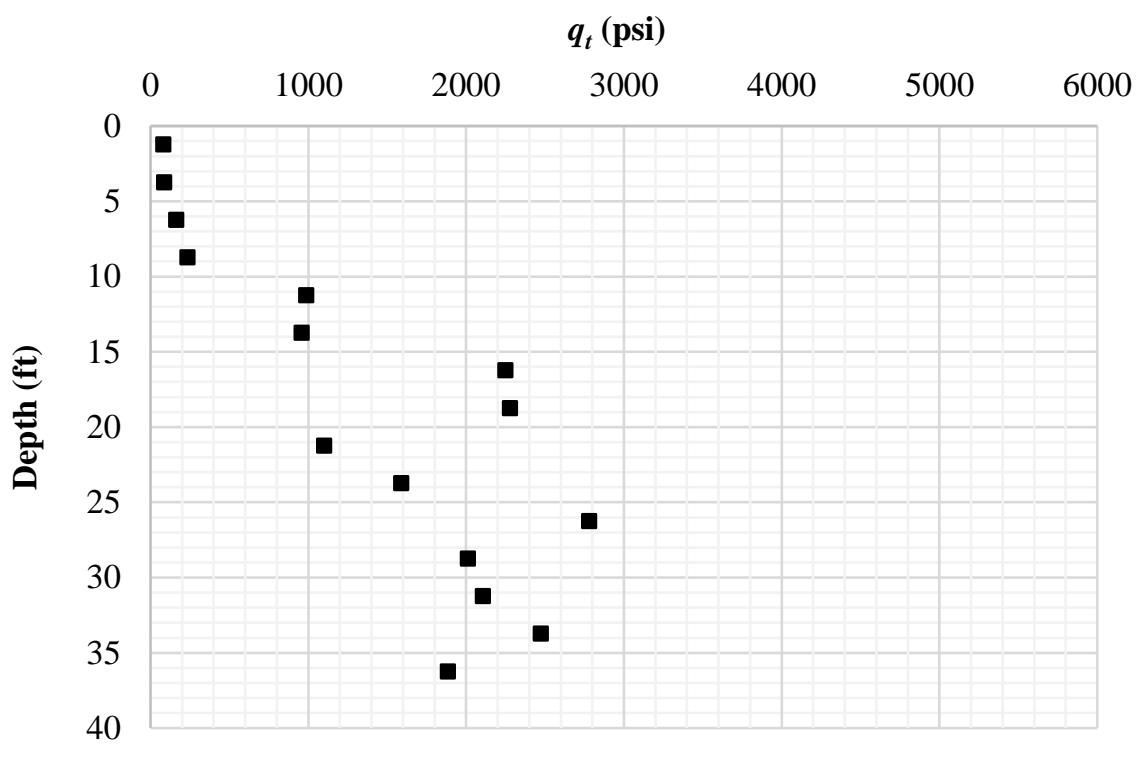

Figure A.67 - Point 170 tip resistance $\left(q_{t}\right)$ vs depth (z). 


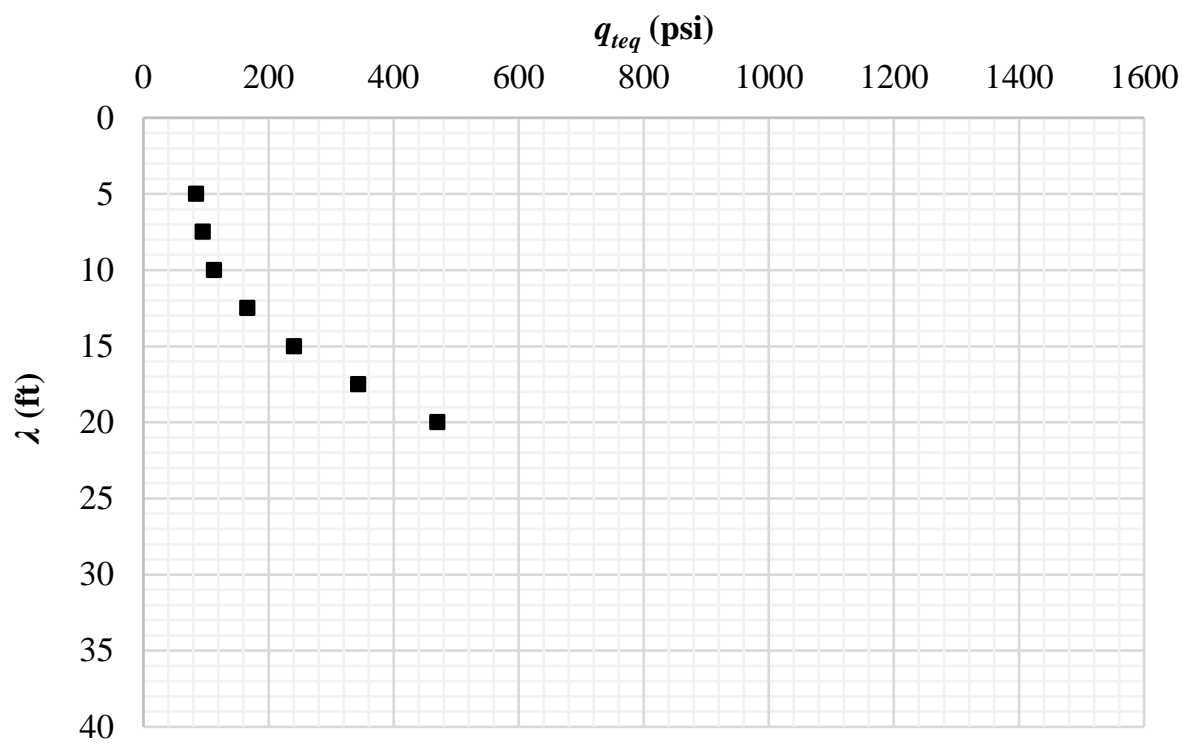

Figure A.68 - Point 170 equivalent tip resistance $\left(q_{t e q}\right)$ vs wavelength (ג).

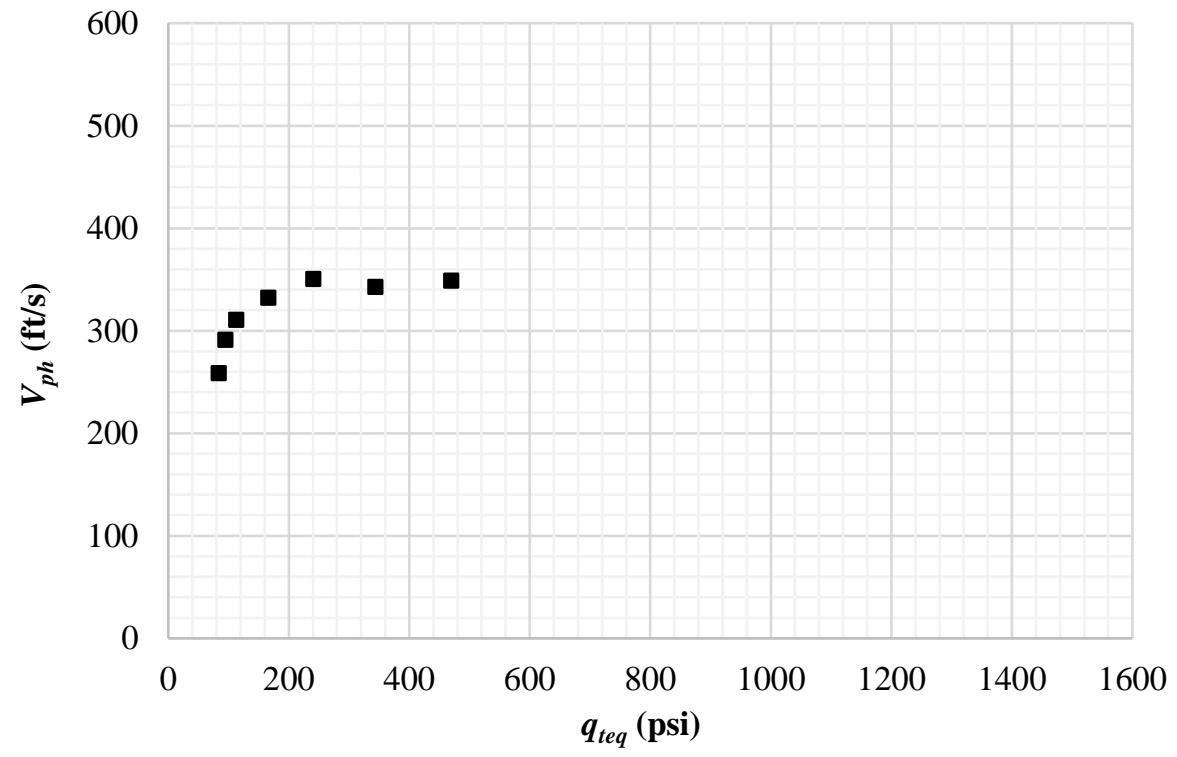

Figure A.69 - Pont 170 equivalent tip resistance $\left(q_{t e q}\right)$ vs phase velocity $\left(V_{p h}\right)$ for the same $\lambda$. 


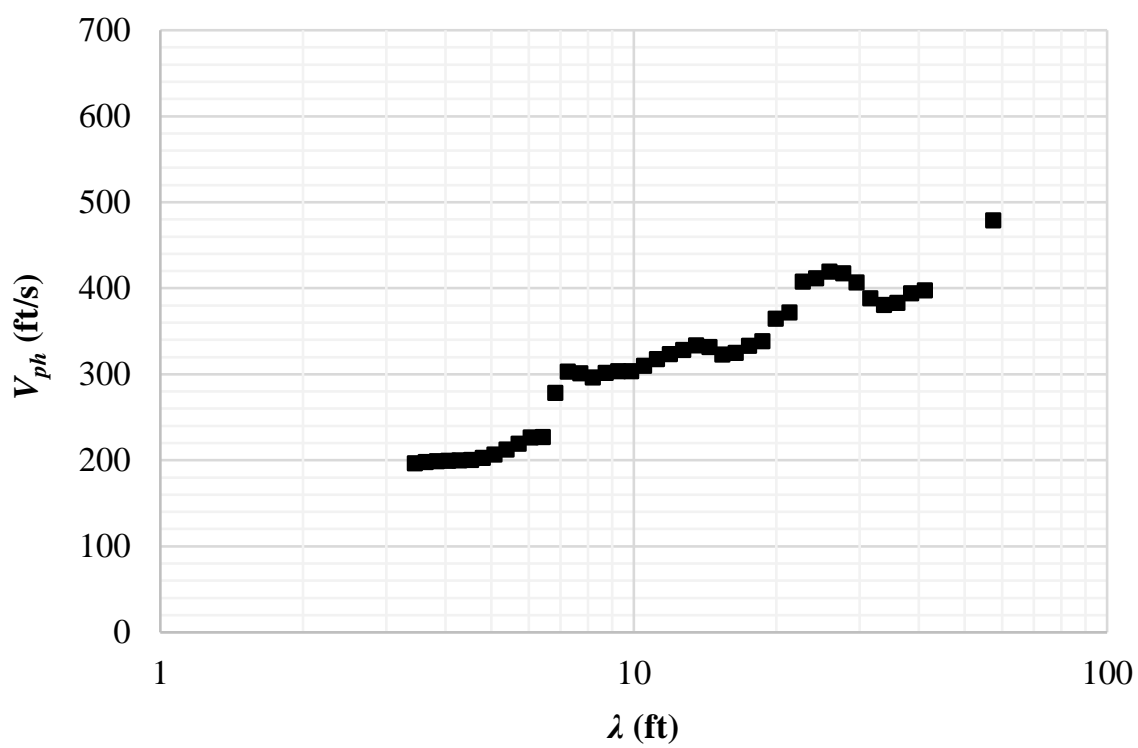

Figure A.70 - Point 180 dispersion curve.

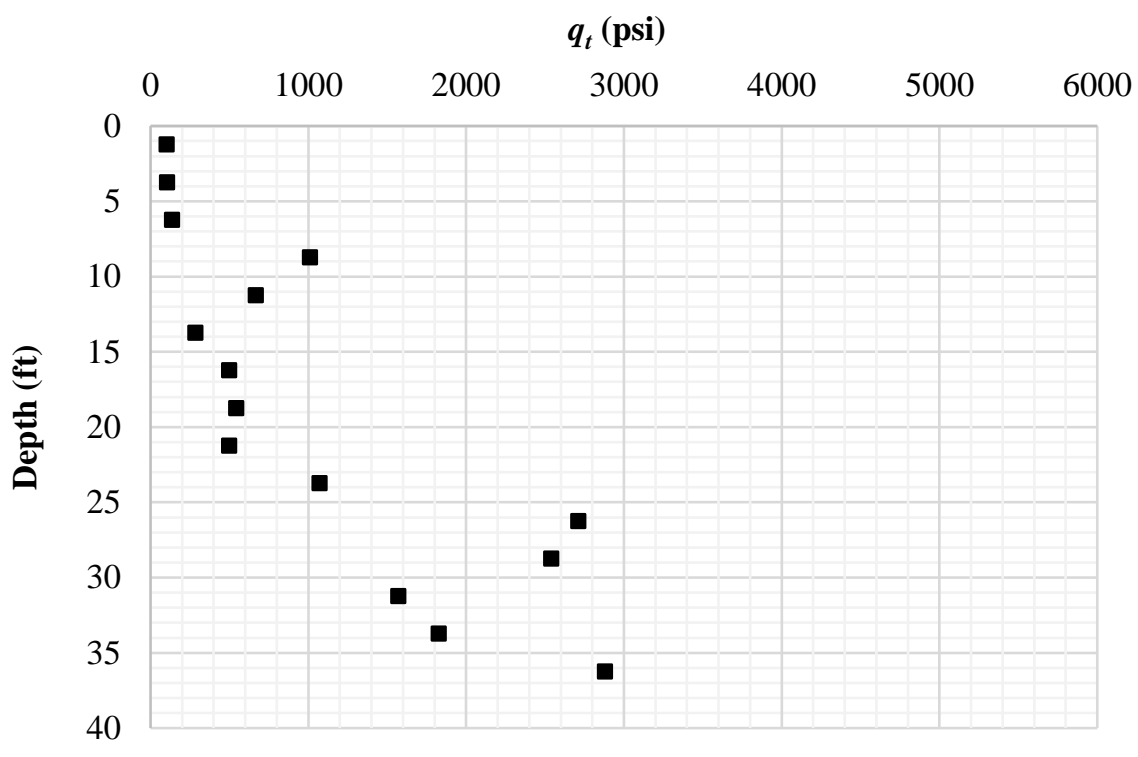

Figure A.71 - Point 180 tip resistance $\left(q_{t}\right)$ vs depth (z). 


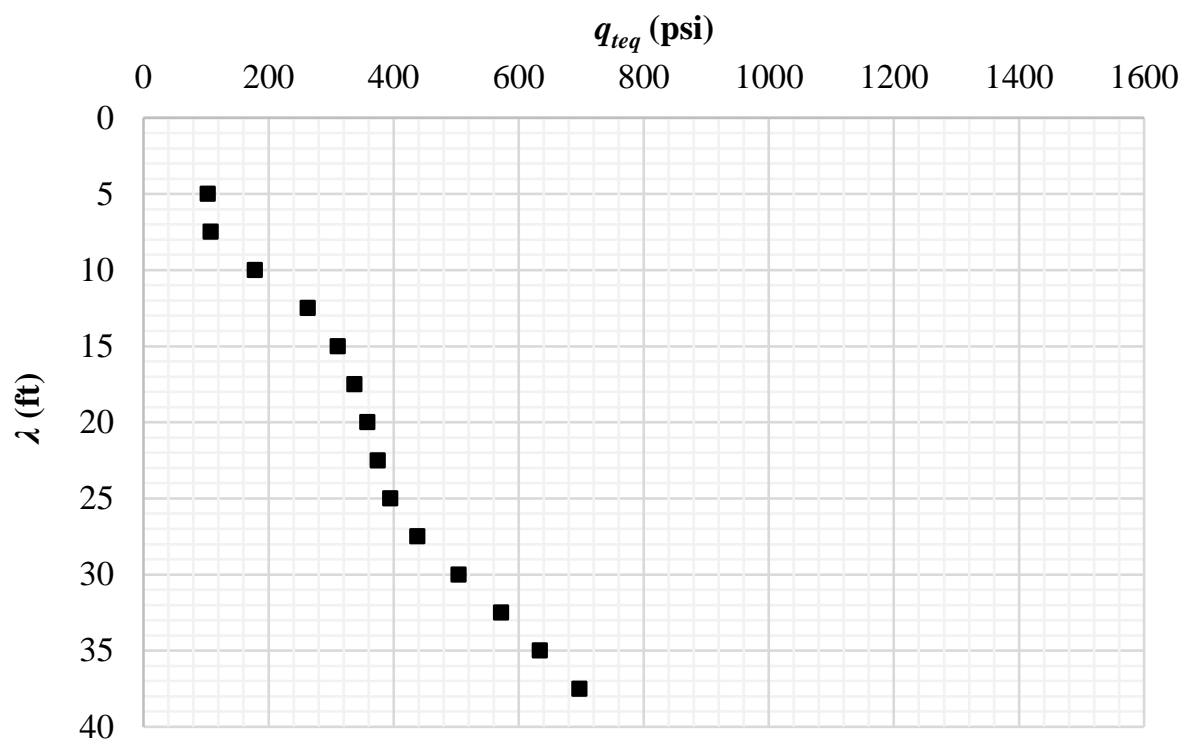

Figure A.72 - Point 180 equivalent tip resistance $\left(q_{t e q}\right)$ vs wavelength $(\lambda)$.

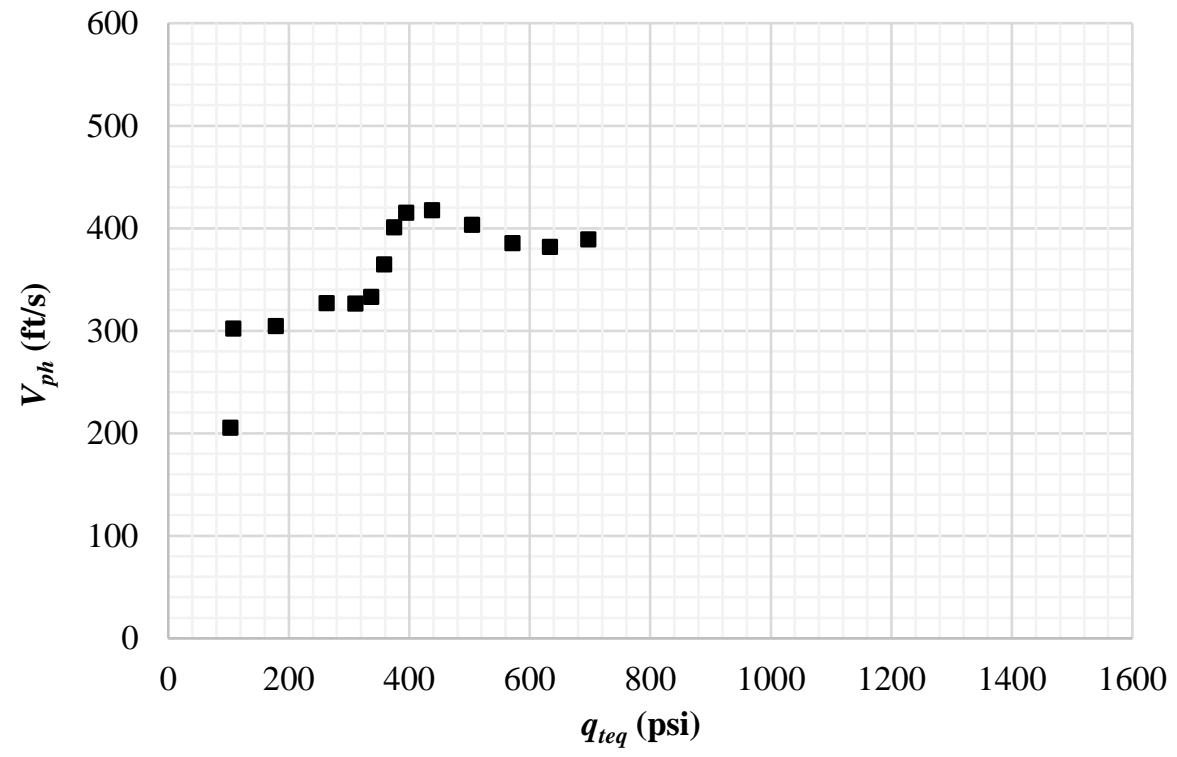

Figure A.73 - Pont 180 equivalent tip resistance $\left(q_{\text {teq }}\right)$ vs phase velocity $\left(V_{p h}\right)$ for the same $\lambda$. 


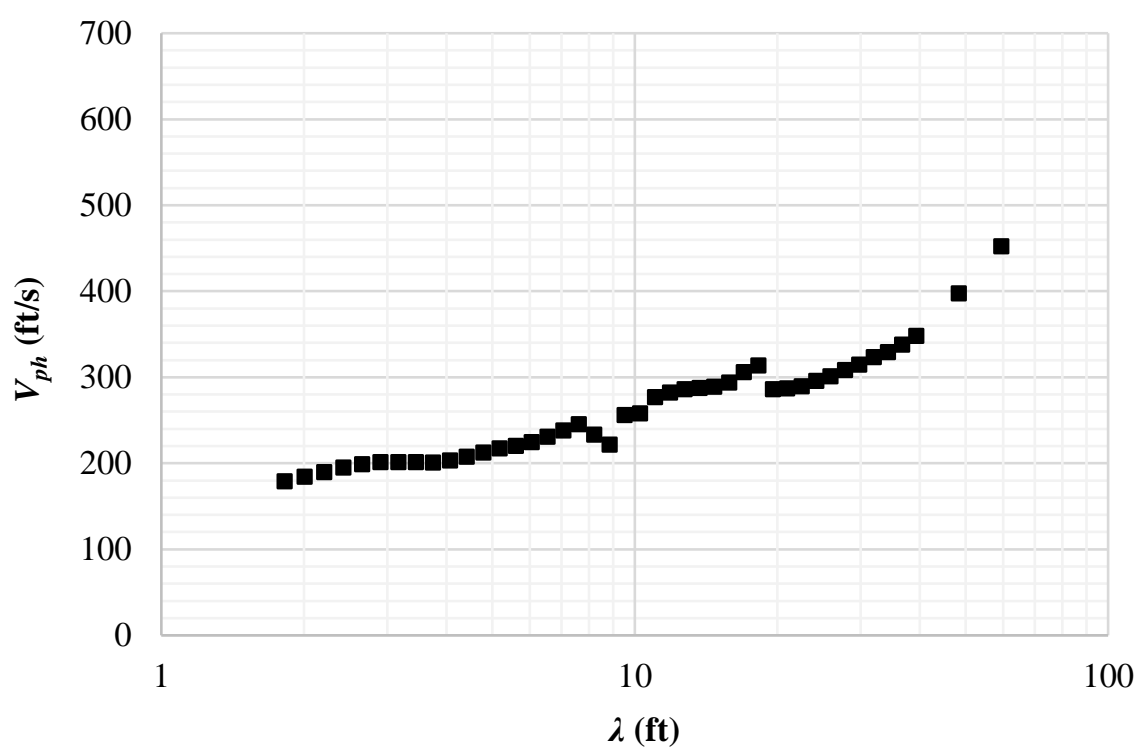

Figure A.74 - Point 190 dispersion curve.

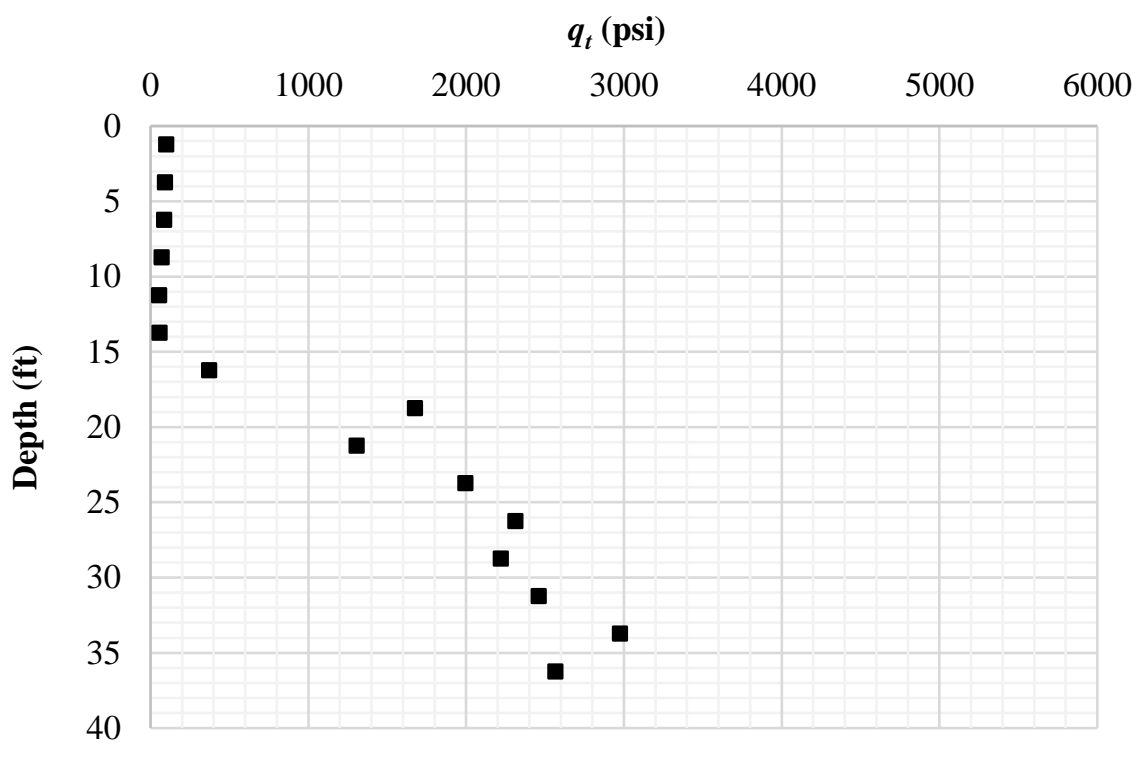

Figure A.75 - Point 190 tip resistance $\left(q_{t}\right)$ vs depth (z). 


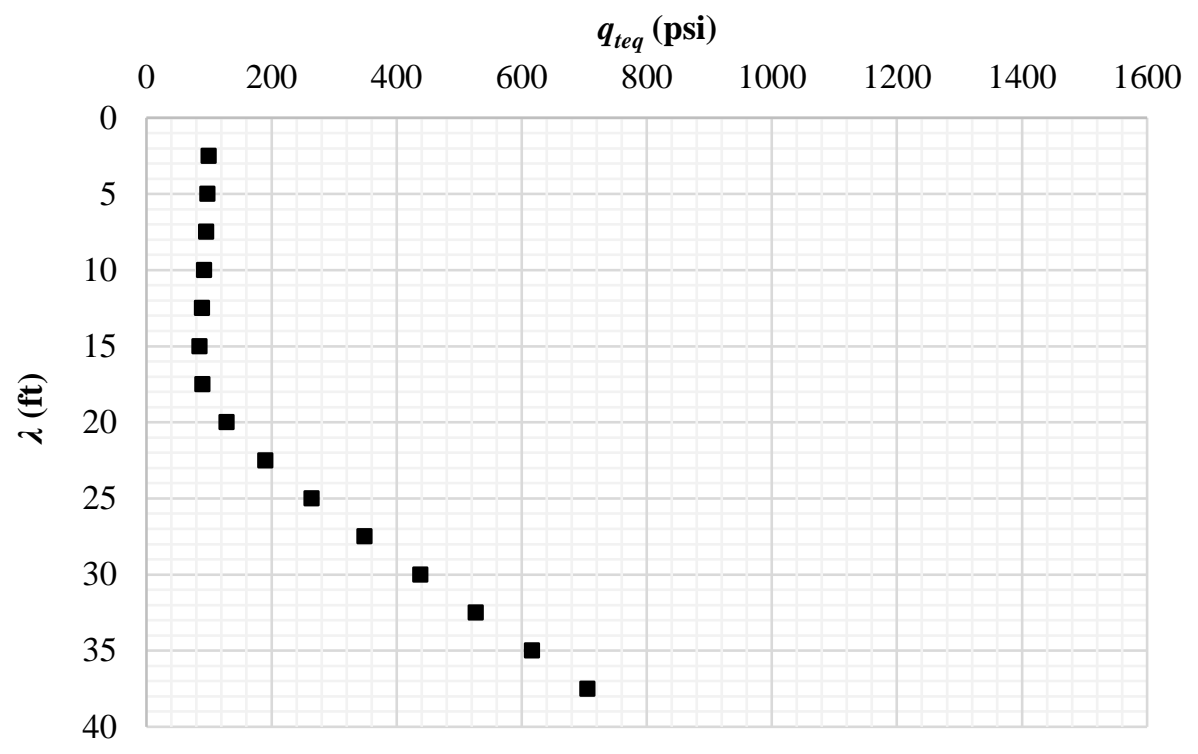

Figure A.76 - Point 190 equivalent tip resistance $\left(q_{t e q}\right)$ vs wavelength (ג).

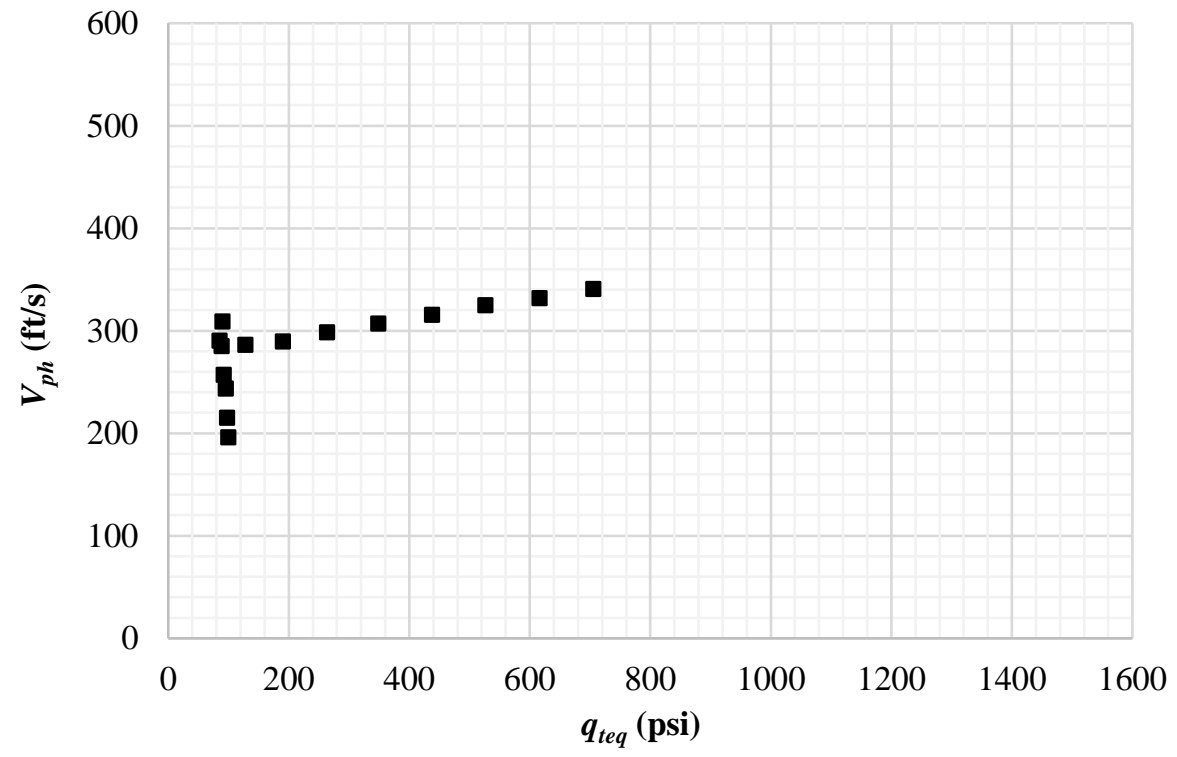

Figure A.77 - Pont 190 equivalent tip resistance $\left(q_{\text {teq }}\right)$ vs phase velocity $\left(V_{p h}\right)$ for the same $\lambda$. 


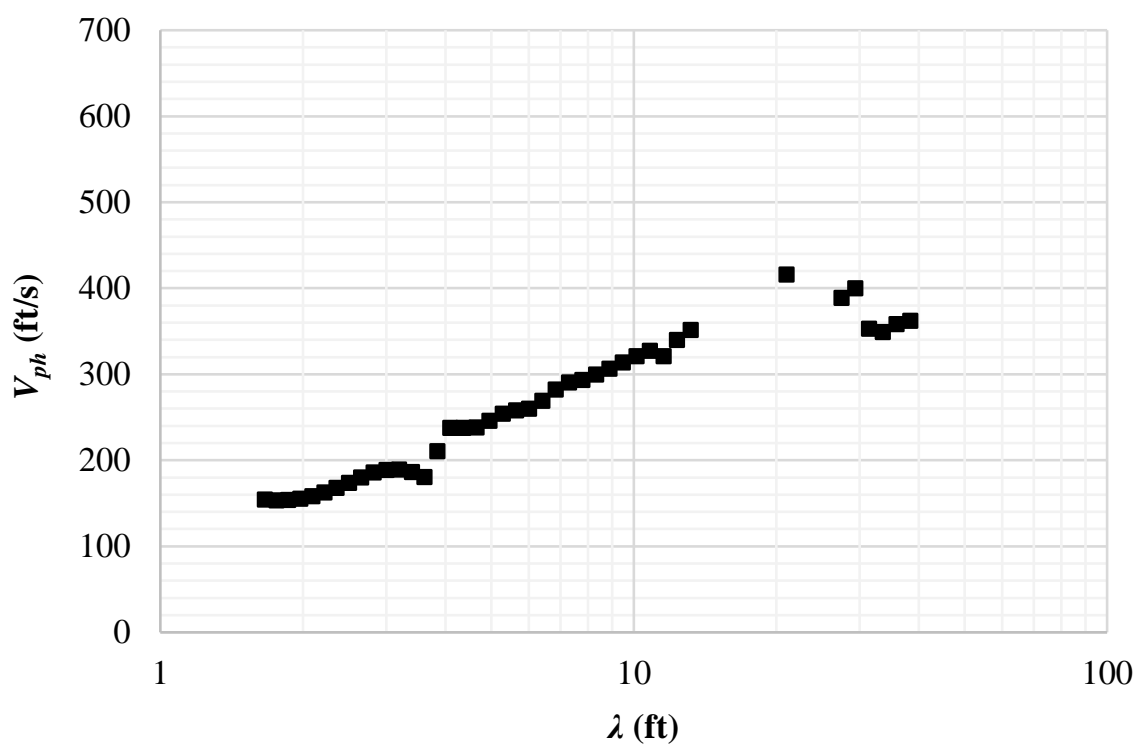

Figure A.78 - Point 192 dispersion curve.

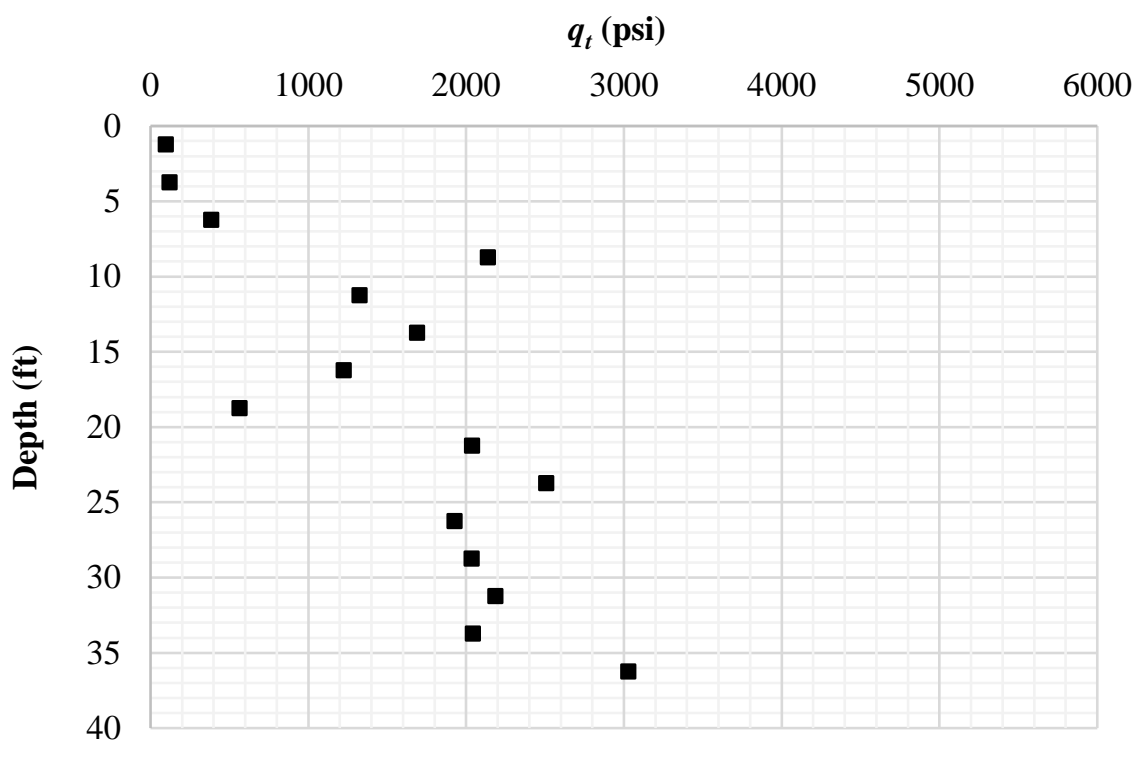

Figure A.79 - Point 192 tip resistance $\left(q_{t}\right)$ vs depth (z). 


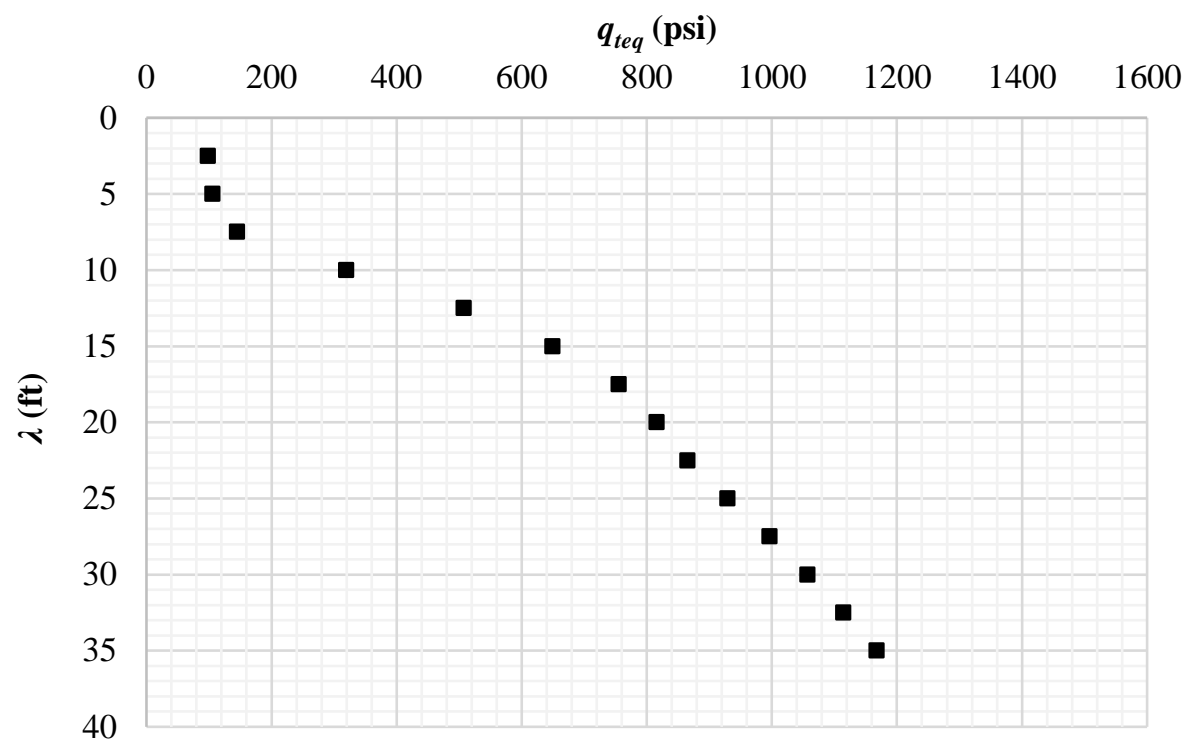

Figure A.80 - Point 192 equivalent tip resistance $\left(q_{t e q}\right)$ vs wavelength (ג).

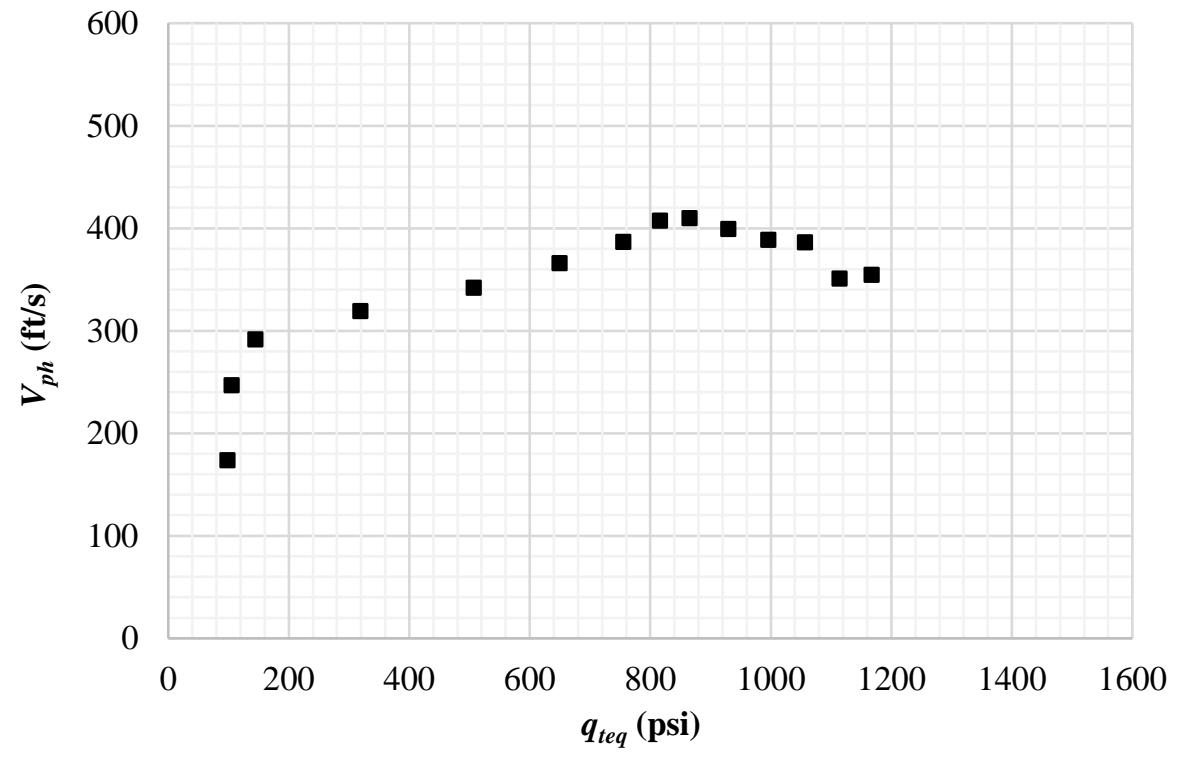

Figure A.81 - Pont 192 equivalent tip resistance $\left(q_{t e q}\right)$ vs phase velocity $\left(V_{p h}\right)$ for the same $\lambda$. 


\section{Group 184}

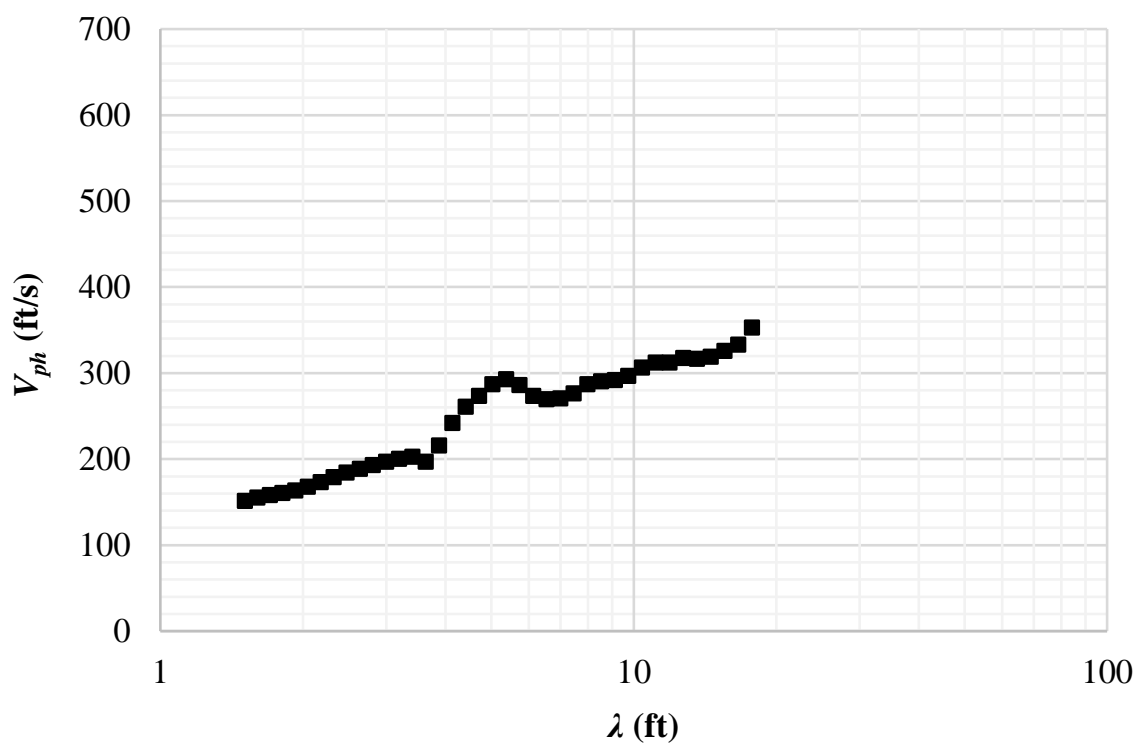

Figure A.82 - Point 172 dispersion curve.

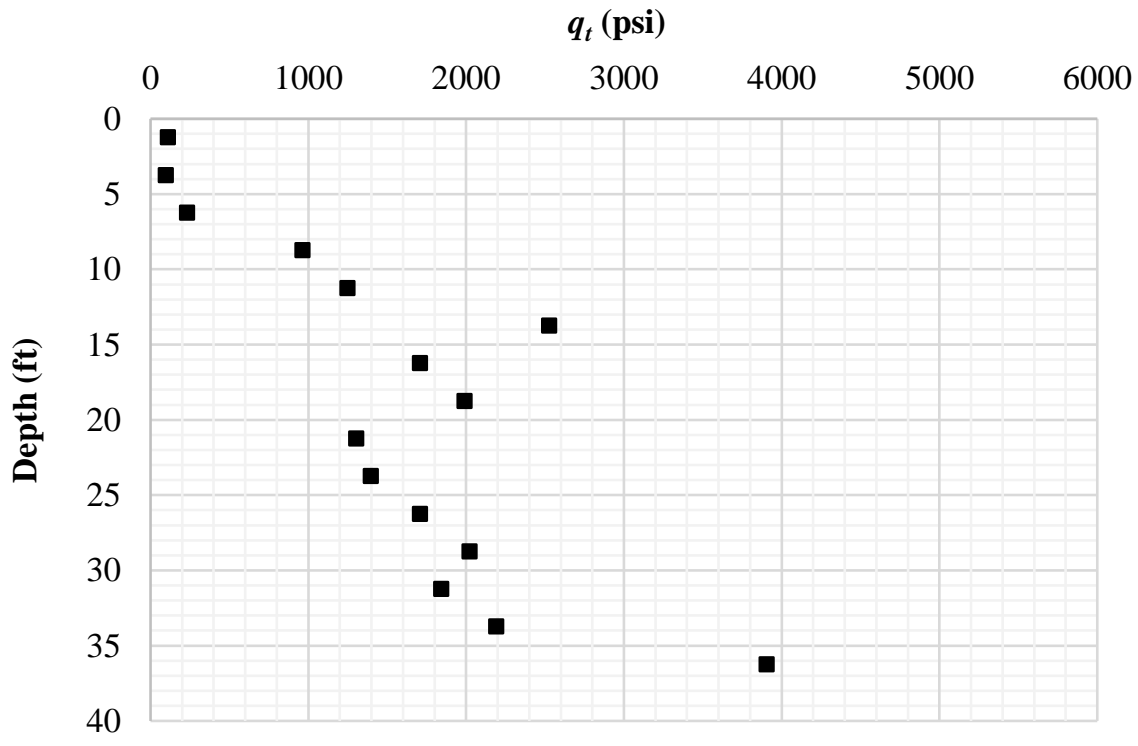

Figure A.83 - Point 172 tip resistance $\left(q_{t}\right)$ vs depth (z). 


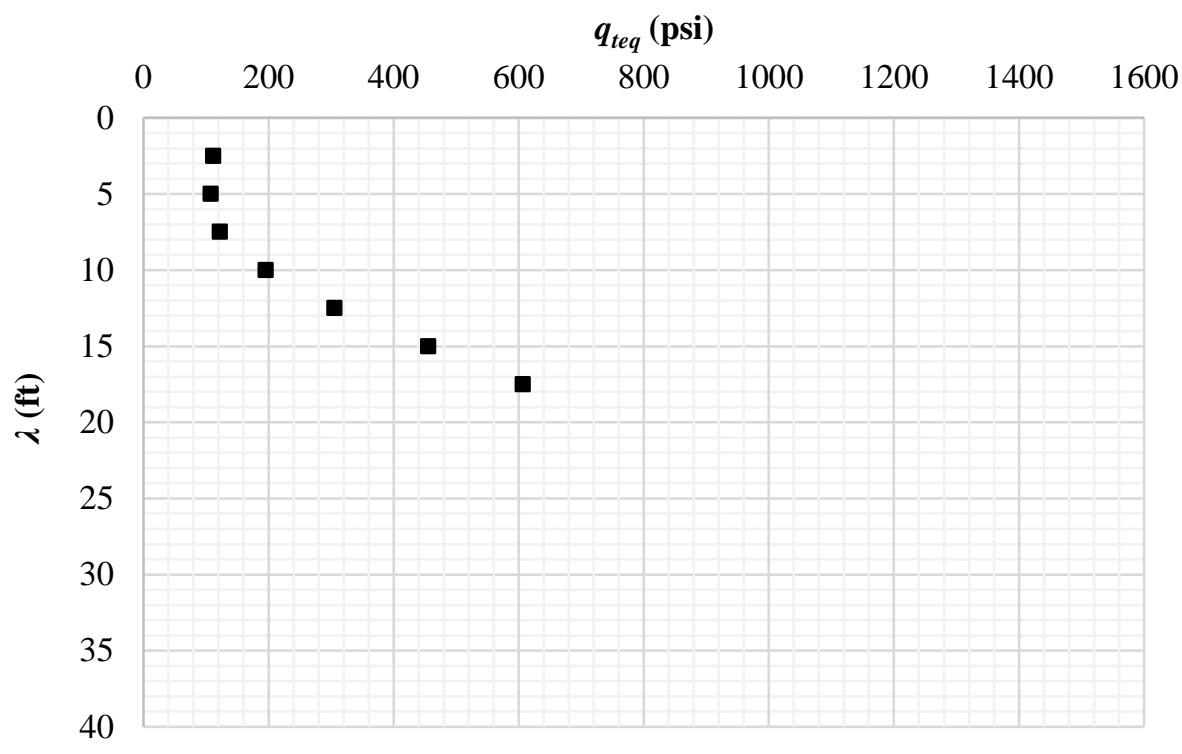

Figure A.84 - Point 172 equivalent tip resistance $\left(q_{t e q}\right)$ vs wavelength (ג).

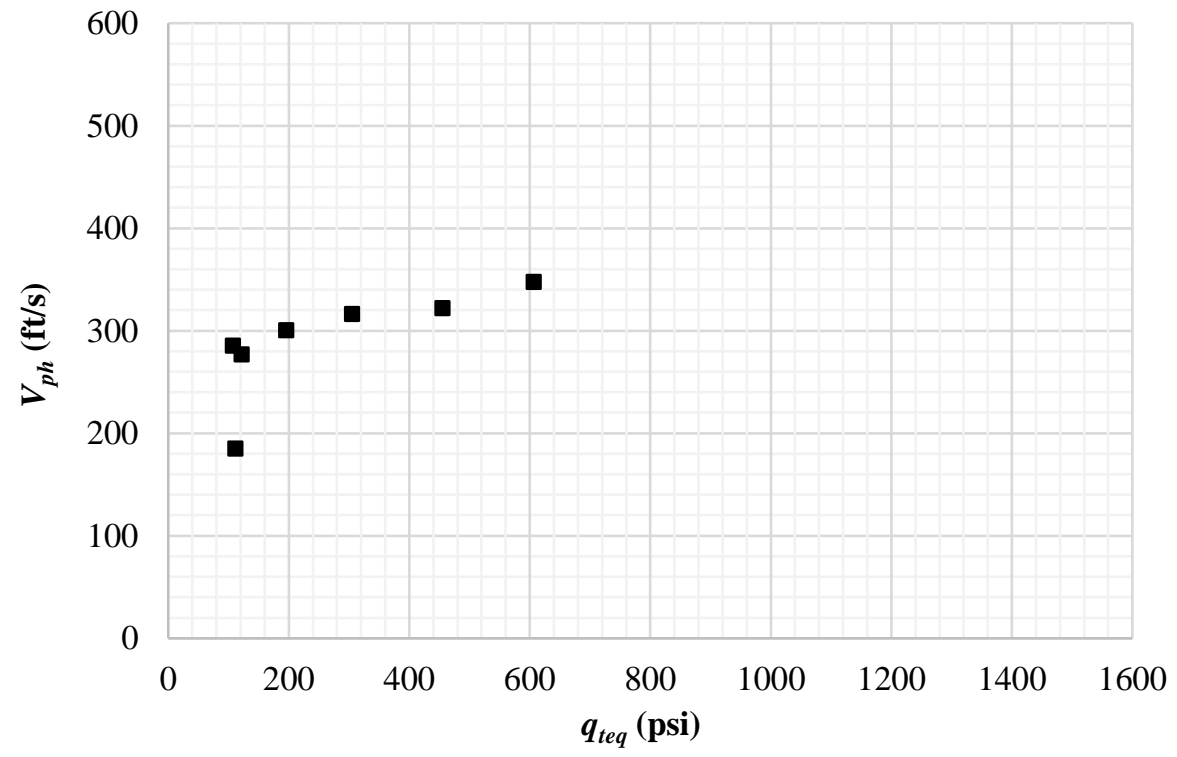

Figure A.85 - Pont 172 equivalent tip resistance $\left(q_{t e q}\right)$ vs phase velocity $\left(V_{p h}\right)$ for the same $\lambda$. 


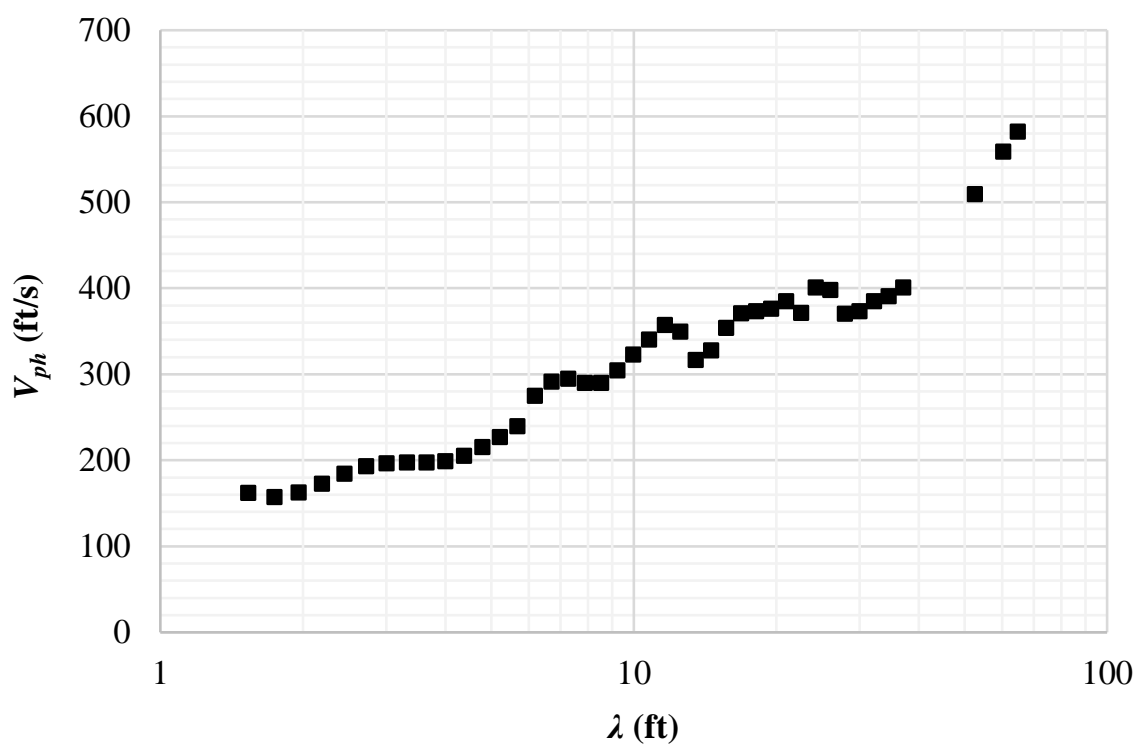

Figure A.86 - Point 174 dispersion curve.

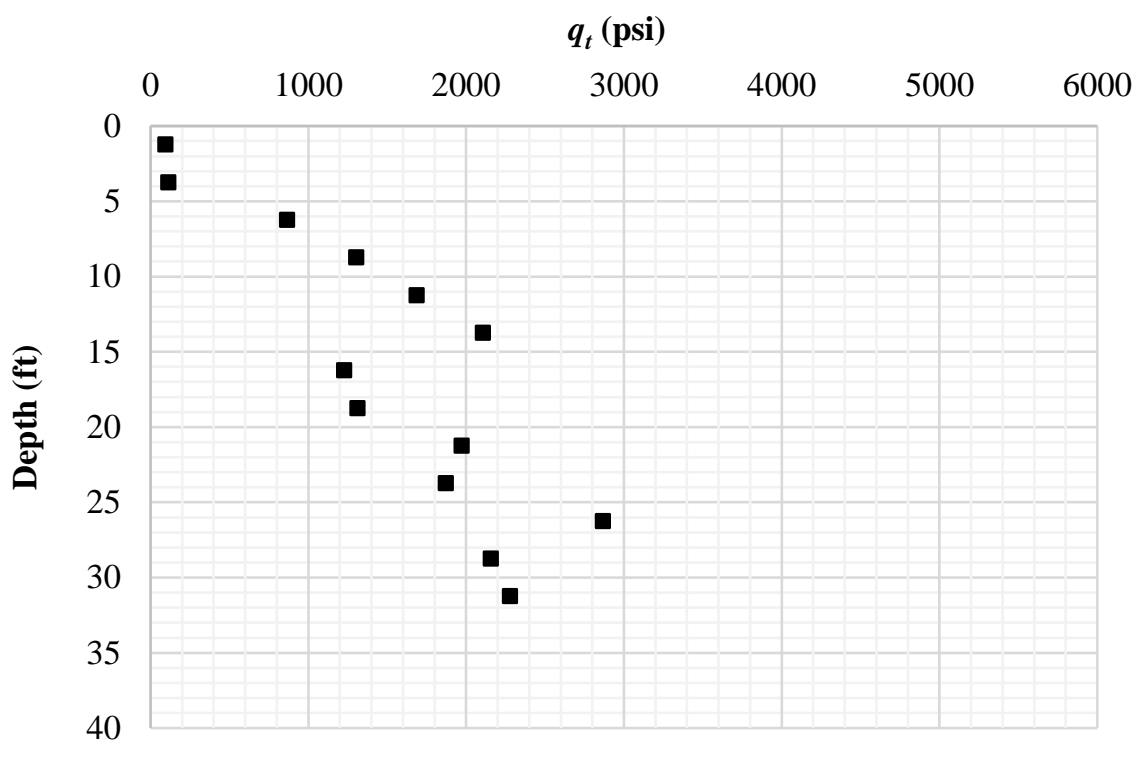

Figure A.87 - Point 174 tip resistance $\left(q_{t}\right)$ vs depth (z). 


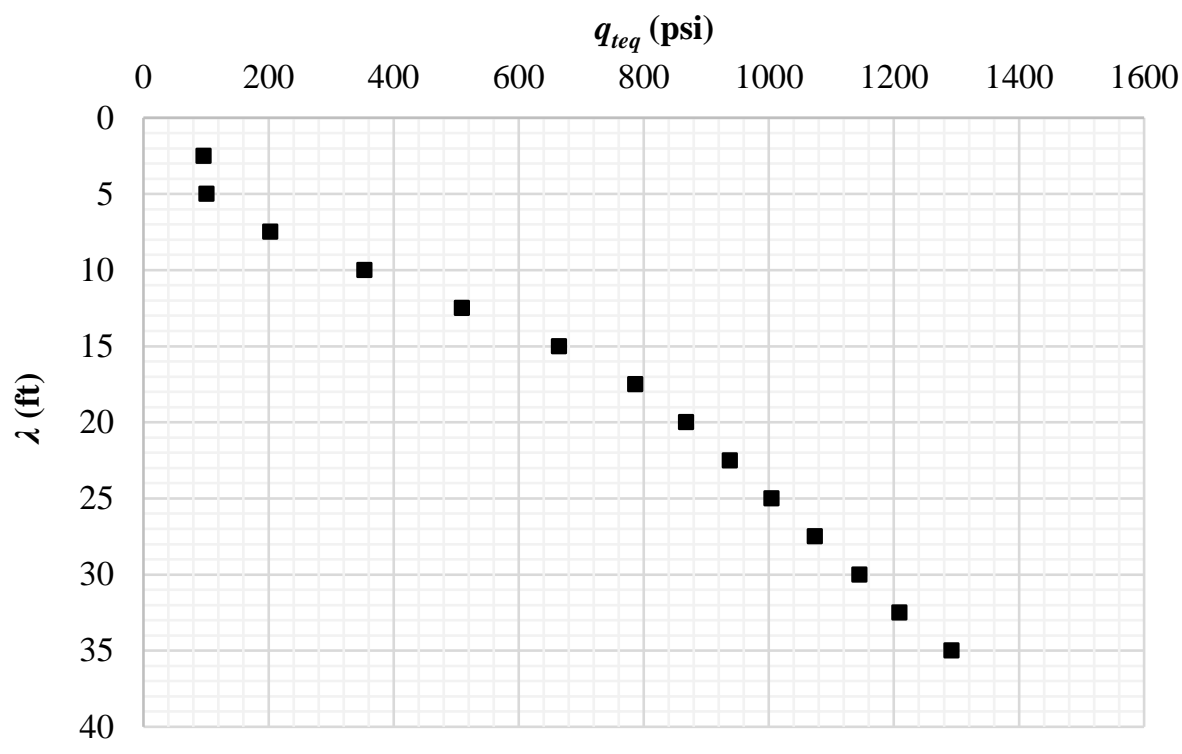

Figure A.88 - Point 174 equivalent tip resistance $\left(q_{t e q}\right)$ vs wavelength (ג).

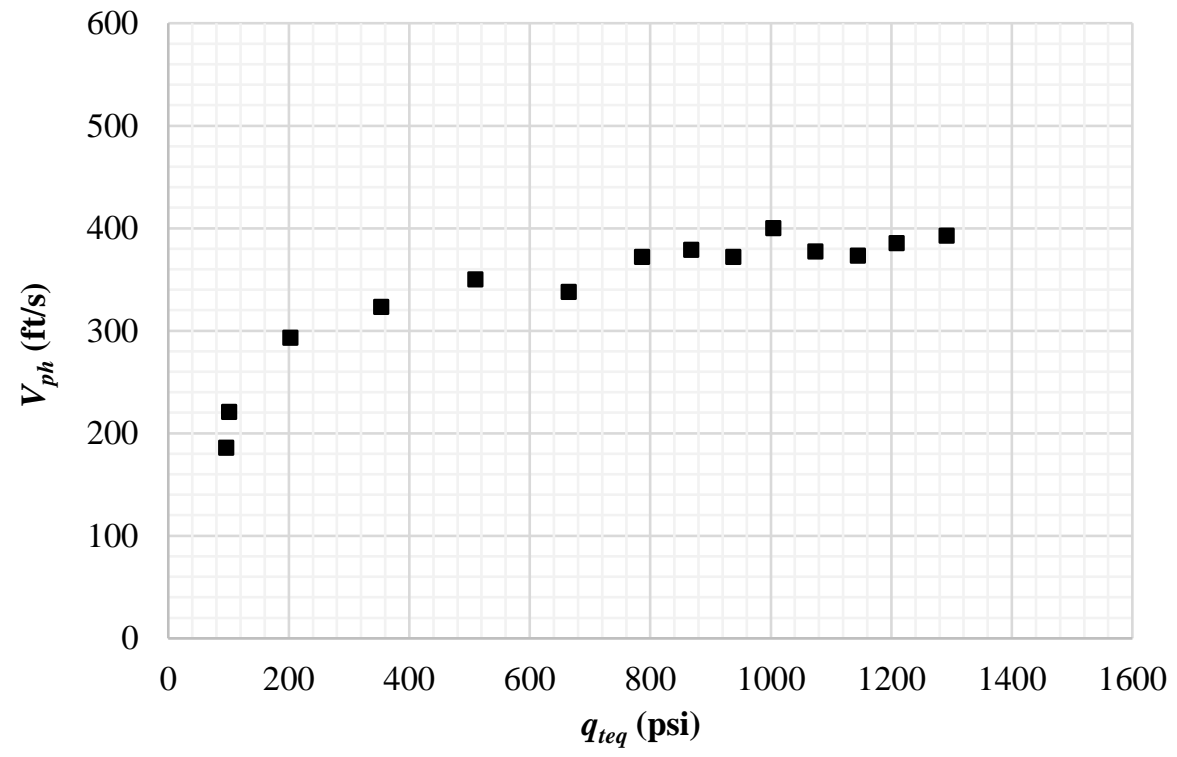

Figure A.89 - Pont 174 equivalent tip resistance $\left(q_{t e q}\right)$ vs phase velocity $\left(V_{p h}\right)$ for the same $\lambda$. 


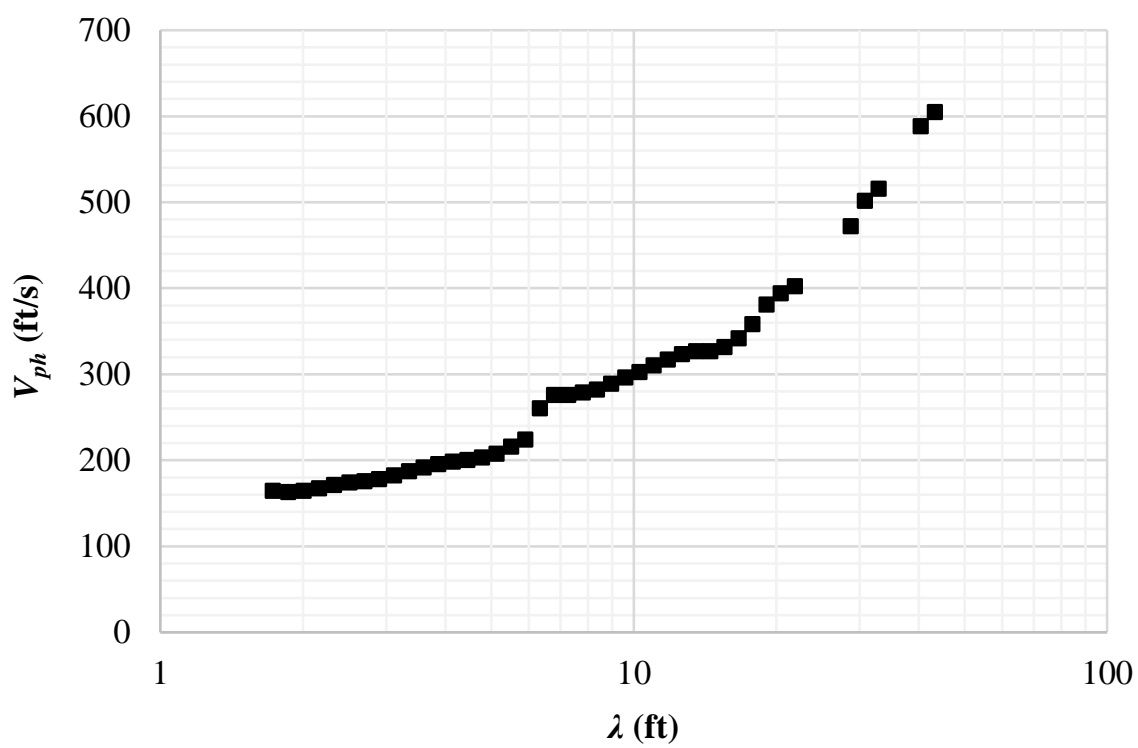

Figure A.90 - Point 184 dispersion curve.

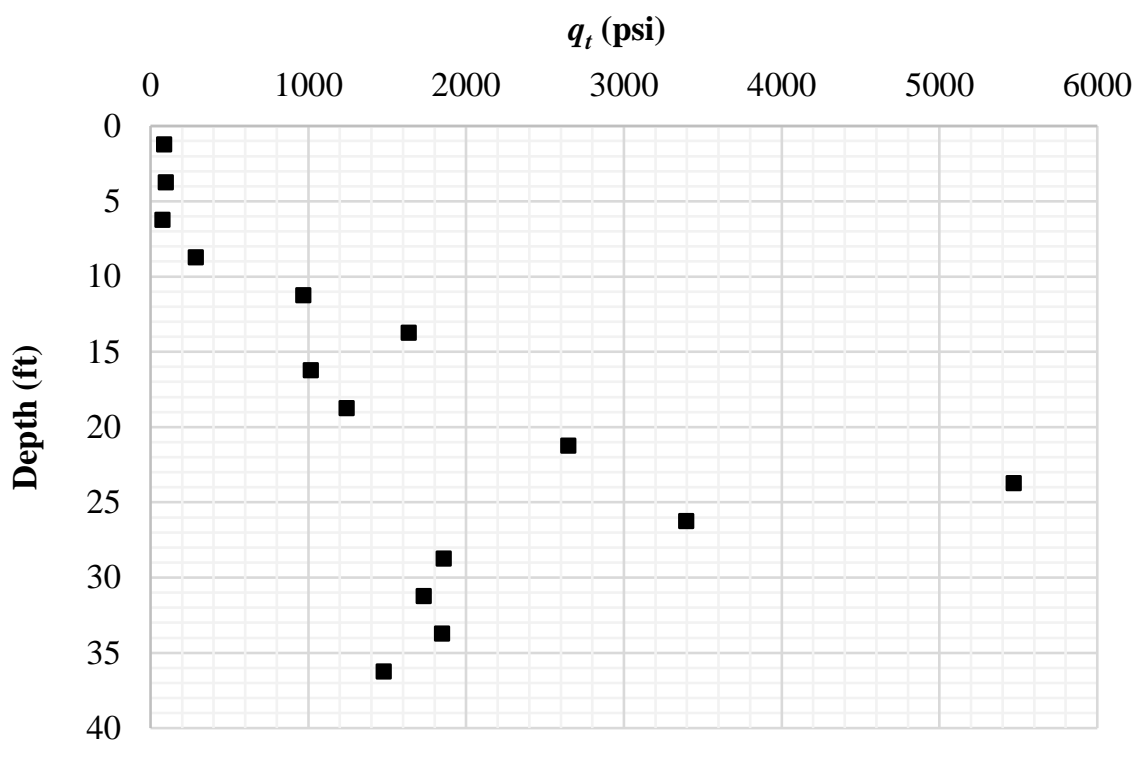

Figure A.91 - Point 184 tip resistance $\left(q_{t}\right)$ vs depth (z). 


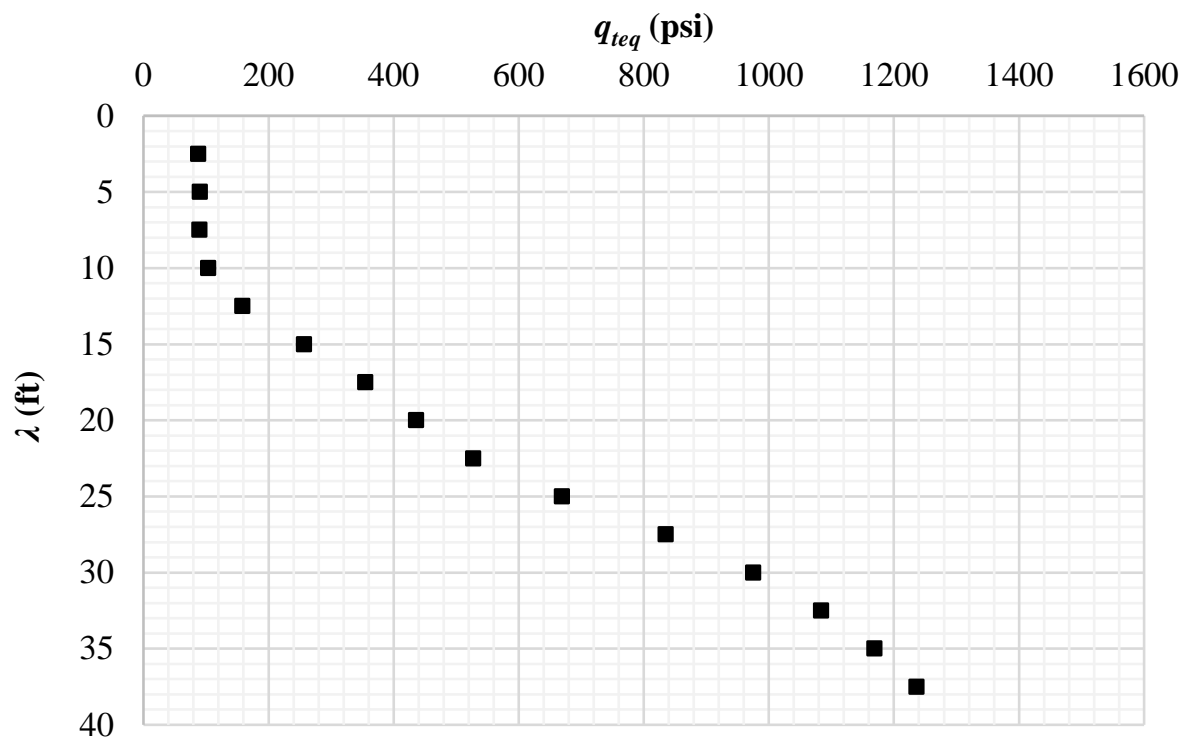

Figure A.92 - Point 184 equivalent tip resistance $\left(q_{t e q}\right)$ vs wavelength (ג).

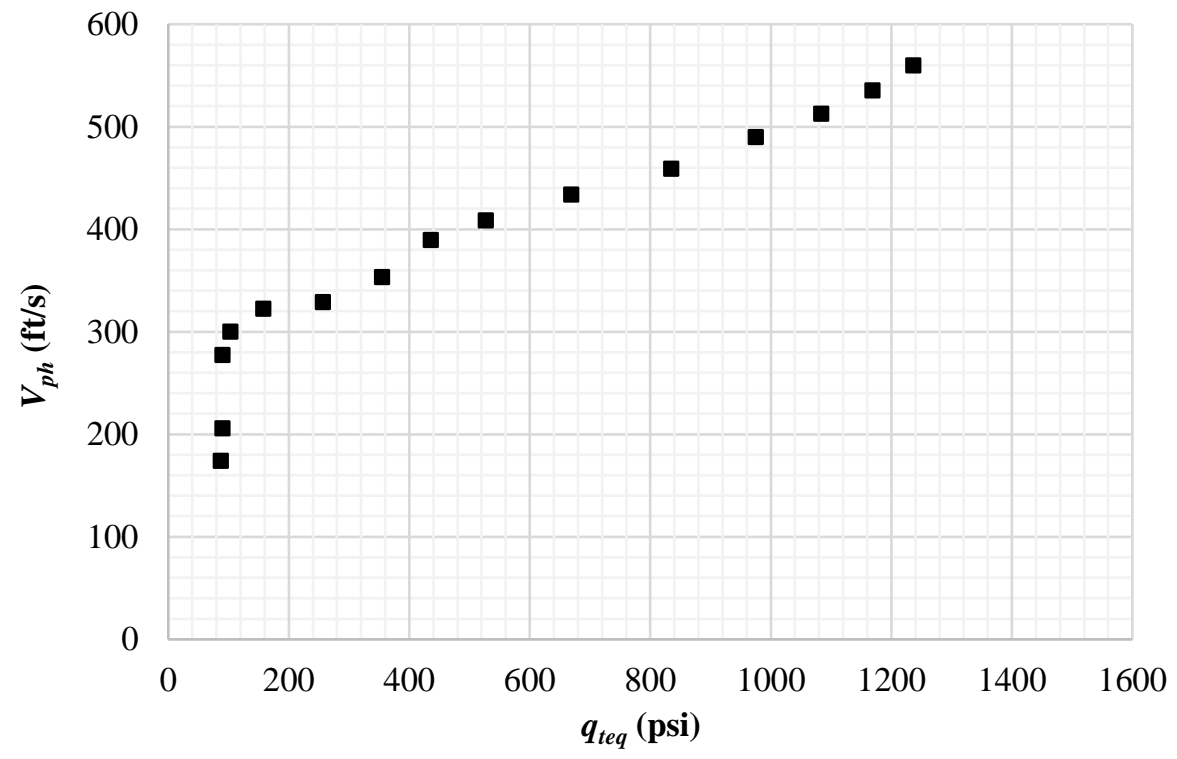

Figure A.93 - Pont 184 equivalent tip resistance $\left(q_{\text {teq }}\right)$ vs phase velocity $\left(V_{p h}\right)$ for the same $\lambda$. 


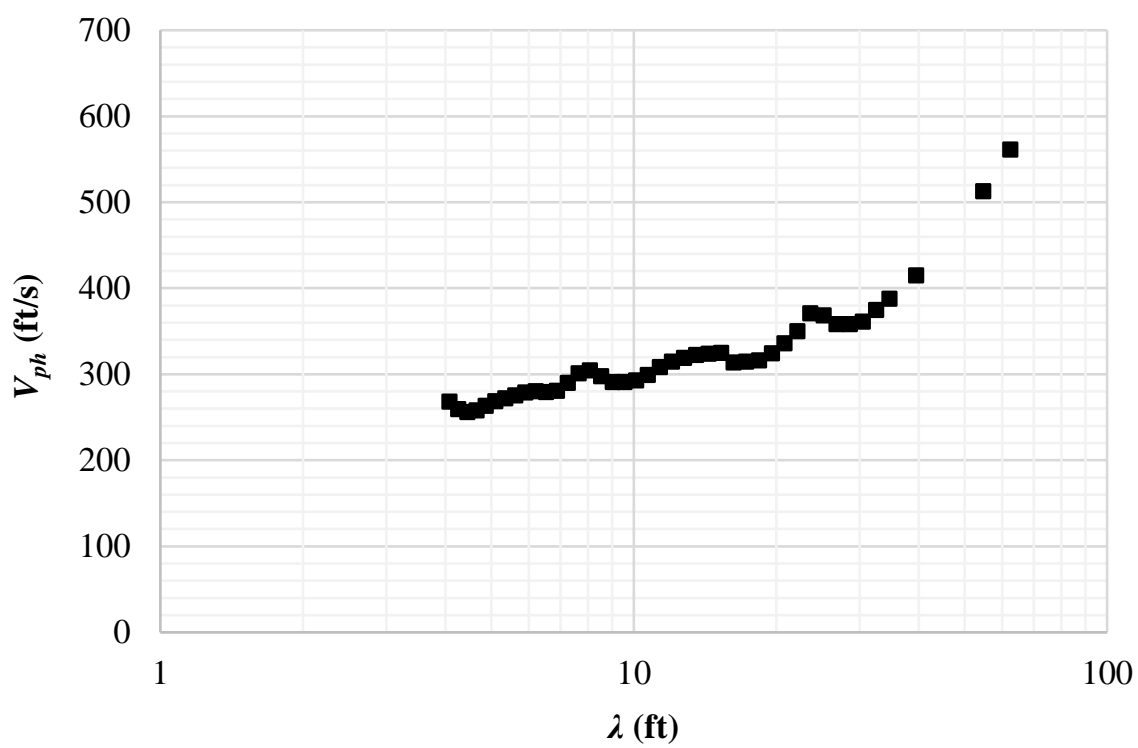

Figure A.94 - Point 194 dispersion curve.

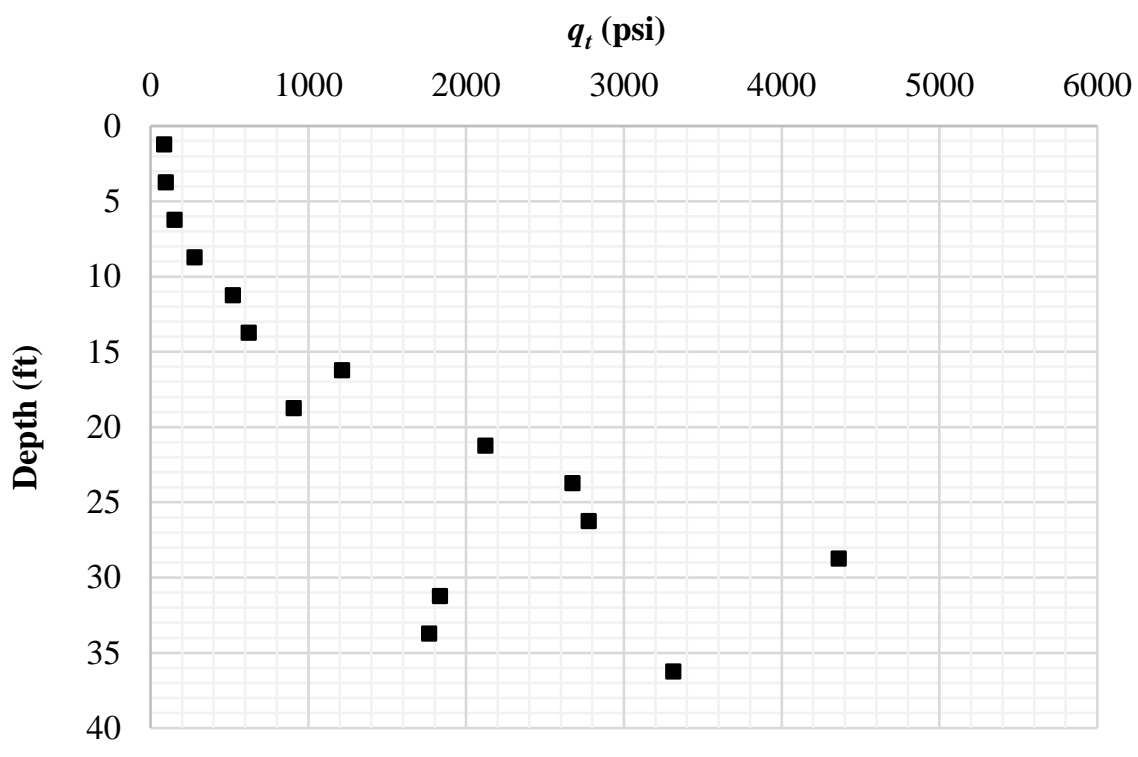

Figure A.95 - Point 194 tip resistance $\left(q_{t}\right)$ vs depth (z). 


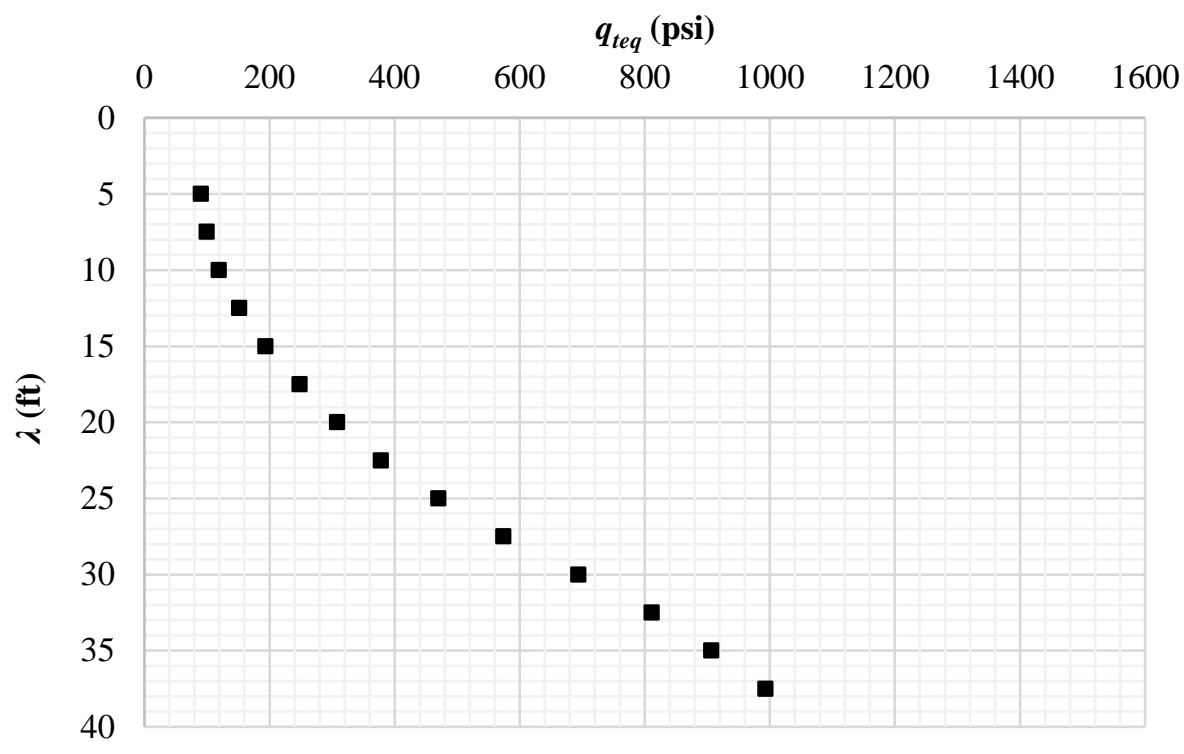

Figure A.96 - Point 194 equivalent tip resistance $\left(q_{t e q}\right)$ vs wavelength (ג).

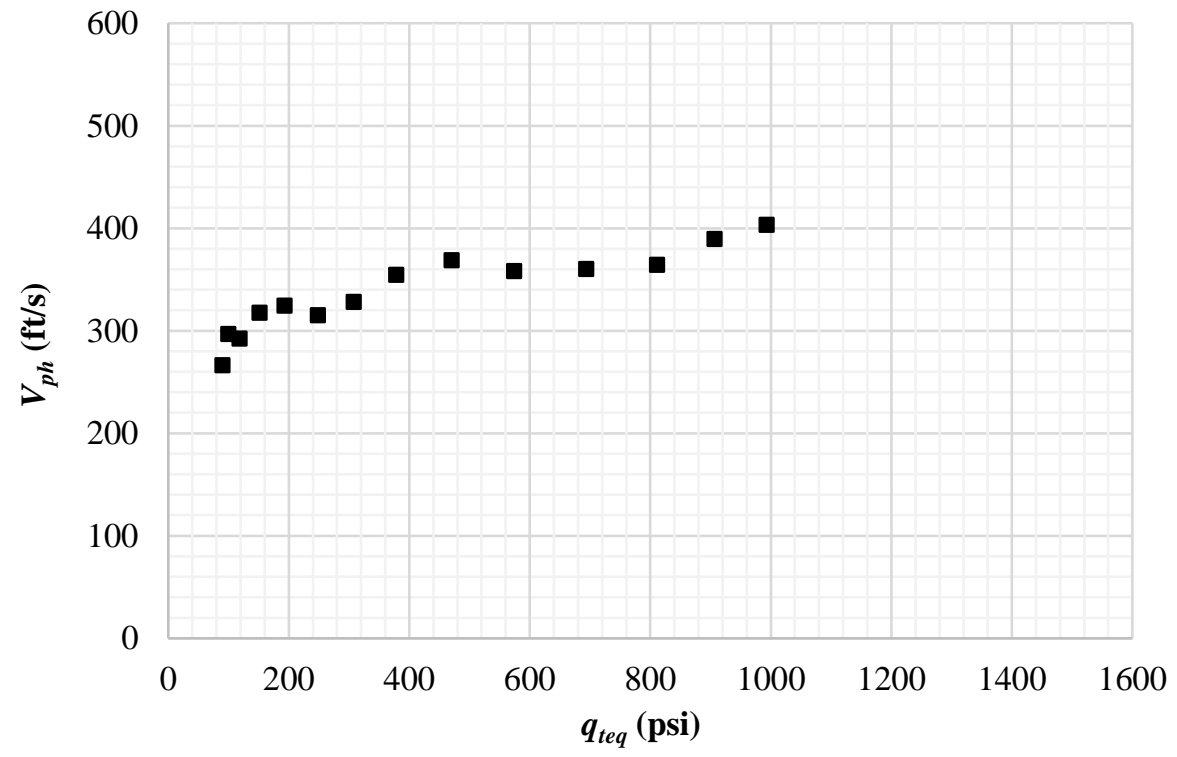

Figure A.97 - Pont 194 equivalent tip resistance $\left(q_{t e q}\right)$ vs phase velocity $\left(V_{p h}\right)$ for the same $\lambda$. 


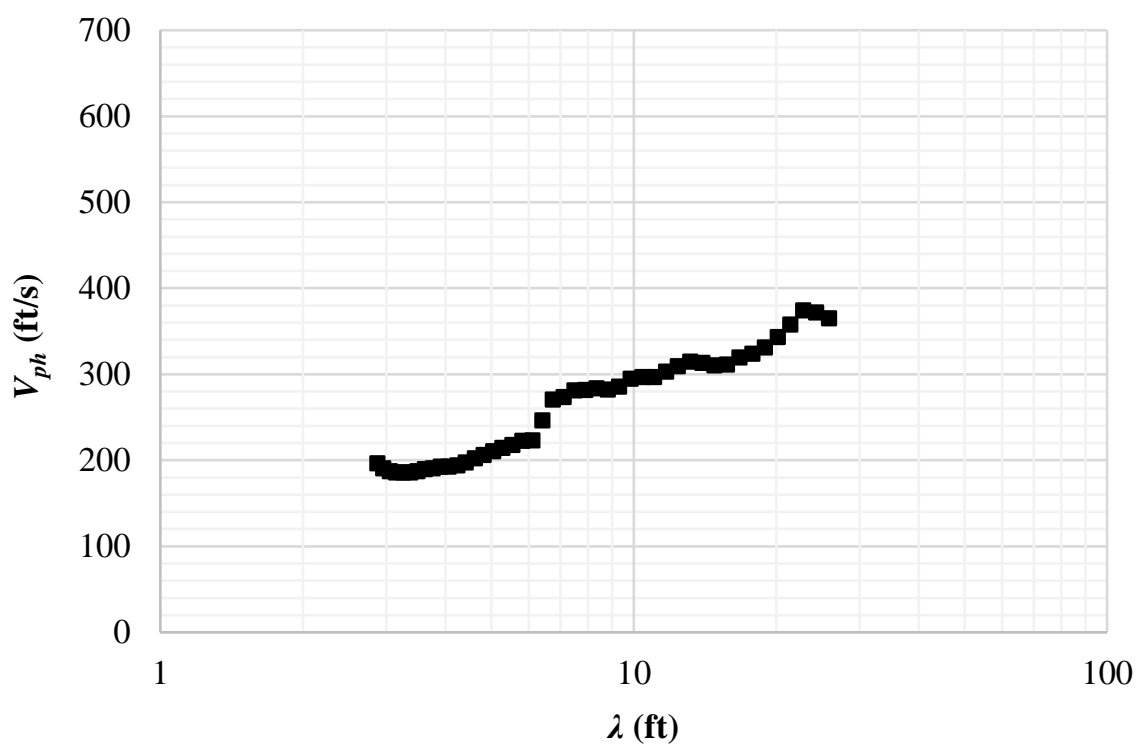

Figure A.98 - Point 196 dispersion curve.

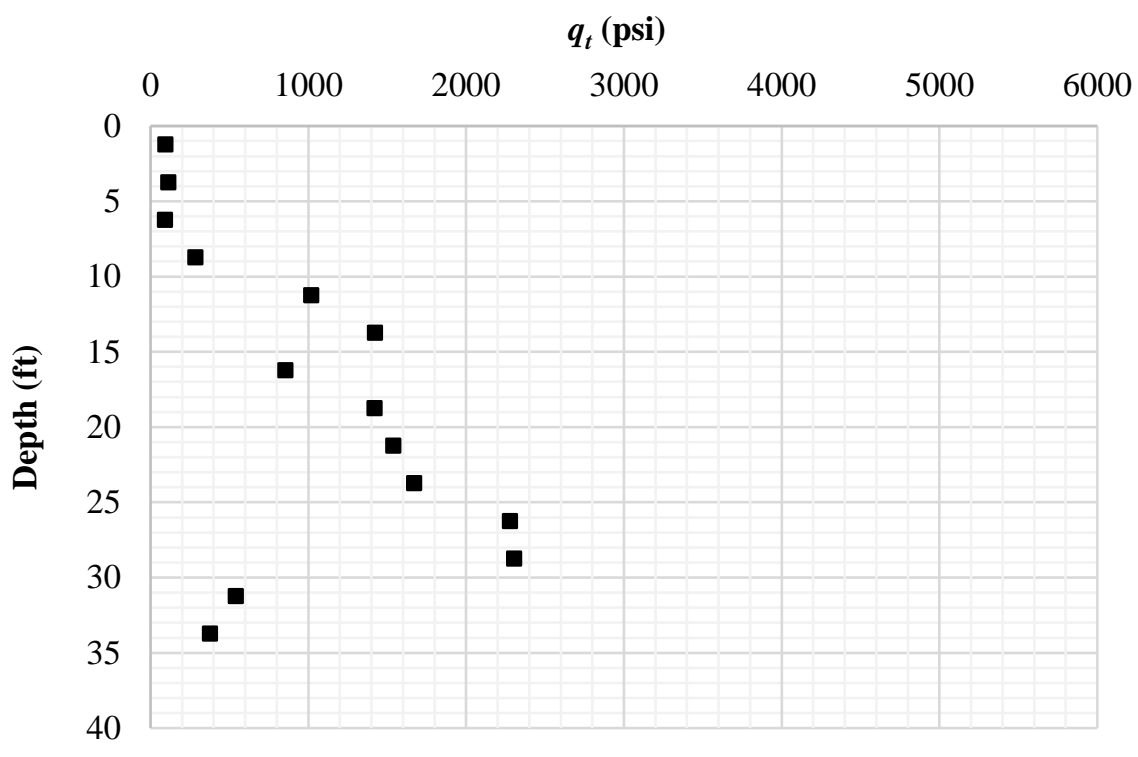

Figure A.99 - Point 196 tip resistance $\left(q_{t}\right)$ vs depth (z). 


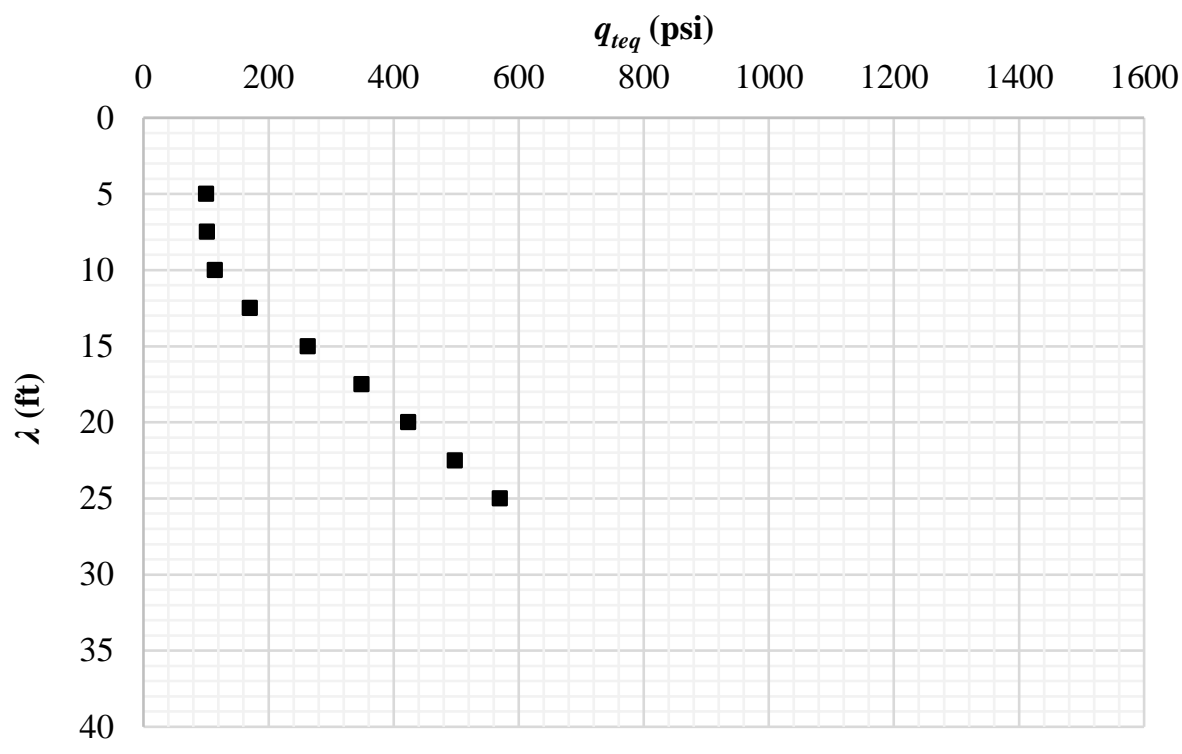

Figure A.100 - Point 196 equivalent tip resistance $\left(q_{t e q}\right)$ vs wavelength (ג).

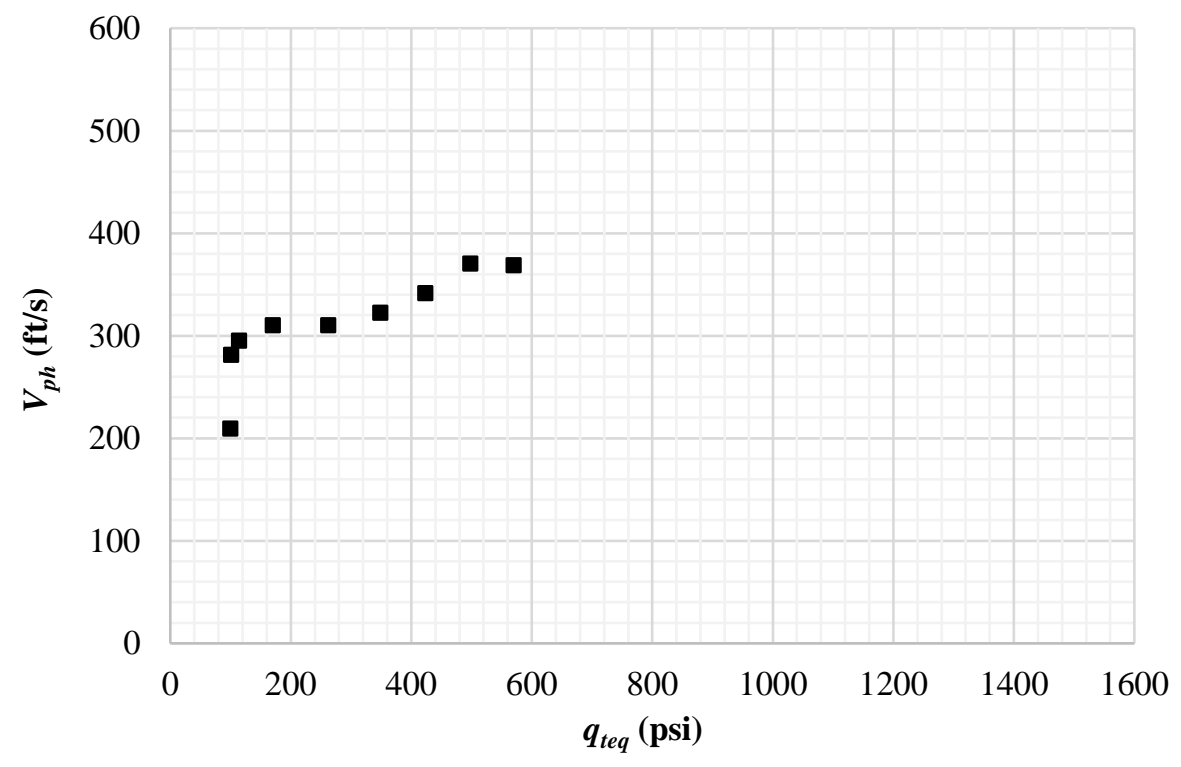

Figure A.101 - Pont 196 equivalent tip resistance $\left(q_{\text {teq }}\right)$ vs phase velocity $\left(V_{p h}\right)$ for the same $\lambda$. 


\section{Appendix B: Group plots}

\section{Group 23}

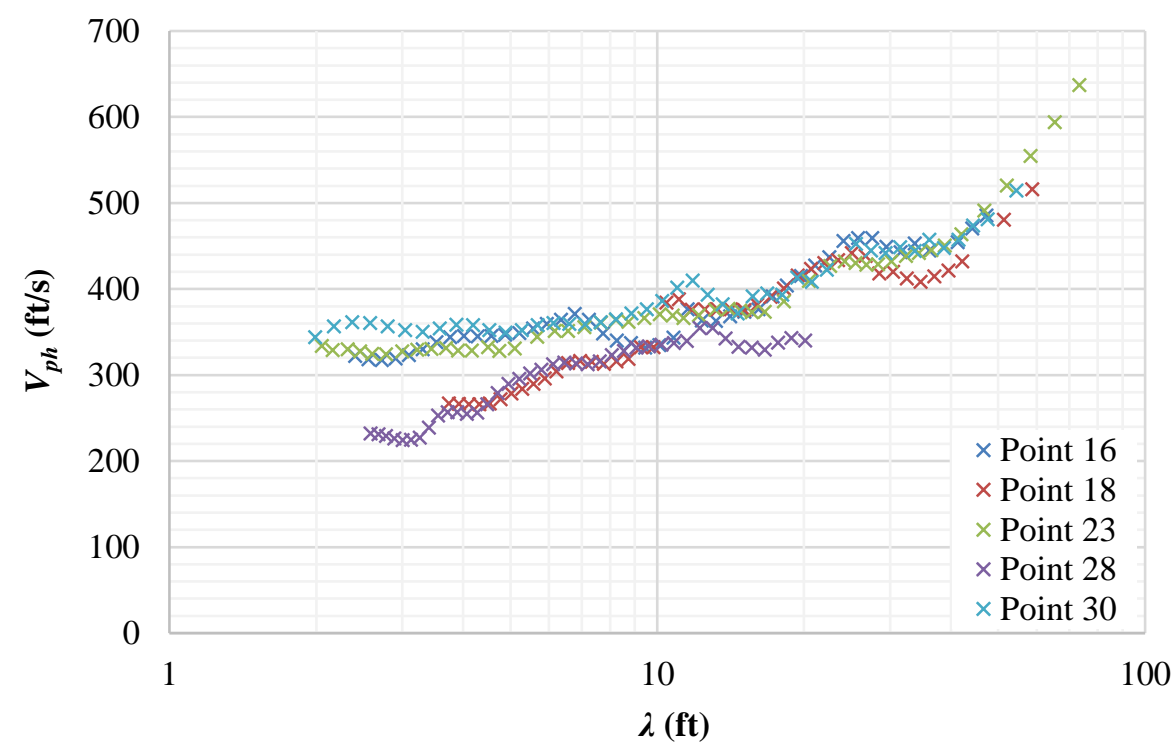

Figure B.1 - Group 23 dispersion curves.

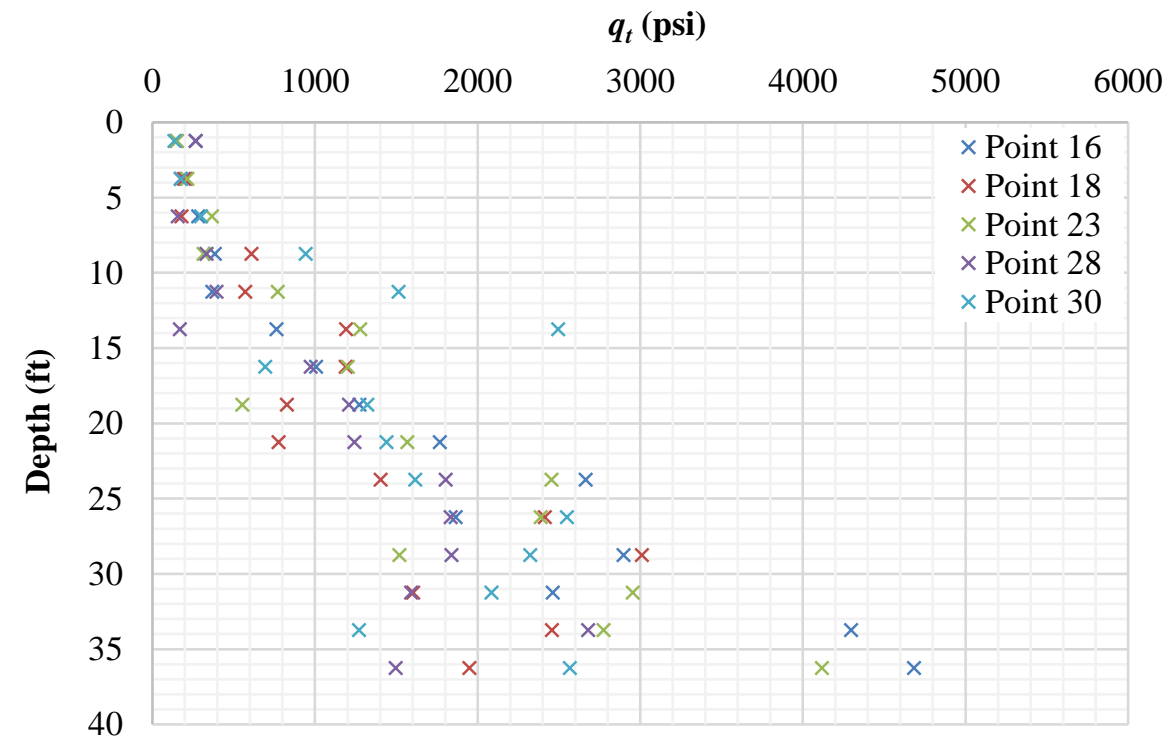

Figure B.2 - Group 23 tip resistance $\left(q_{t}\right)$ vs depth $(\mathrm{z})$. 


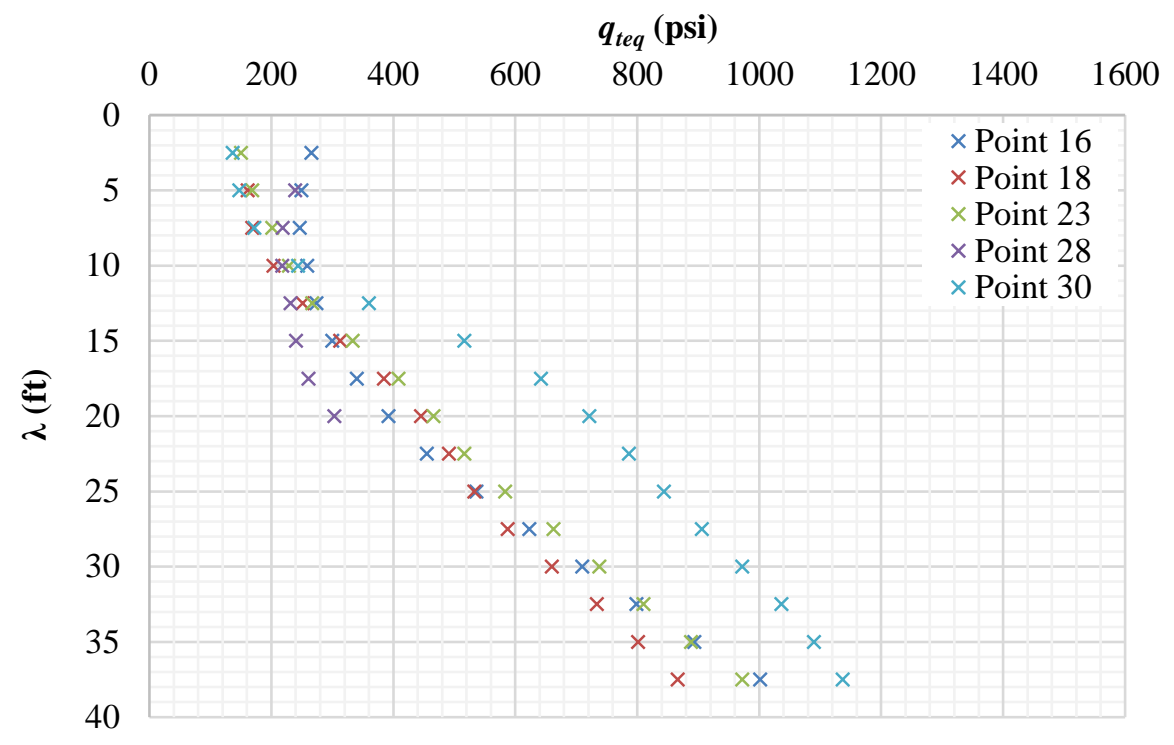

Figure B.3 - Group 23 equivalent tip resistance $\left(q_{t e q}\right)$ vs wavelength ( $\lambda$ ).

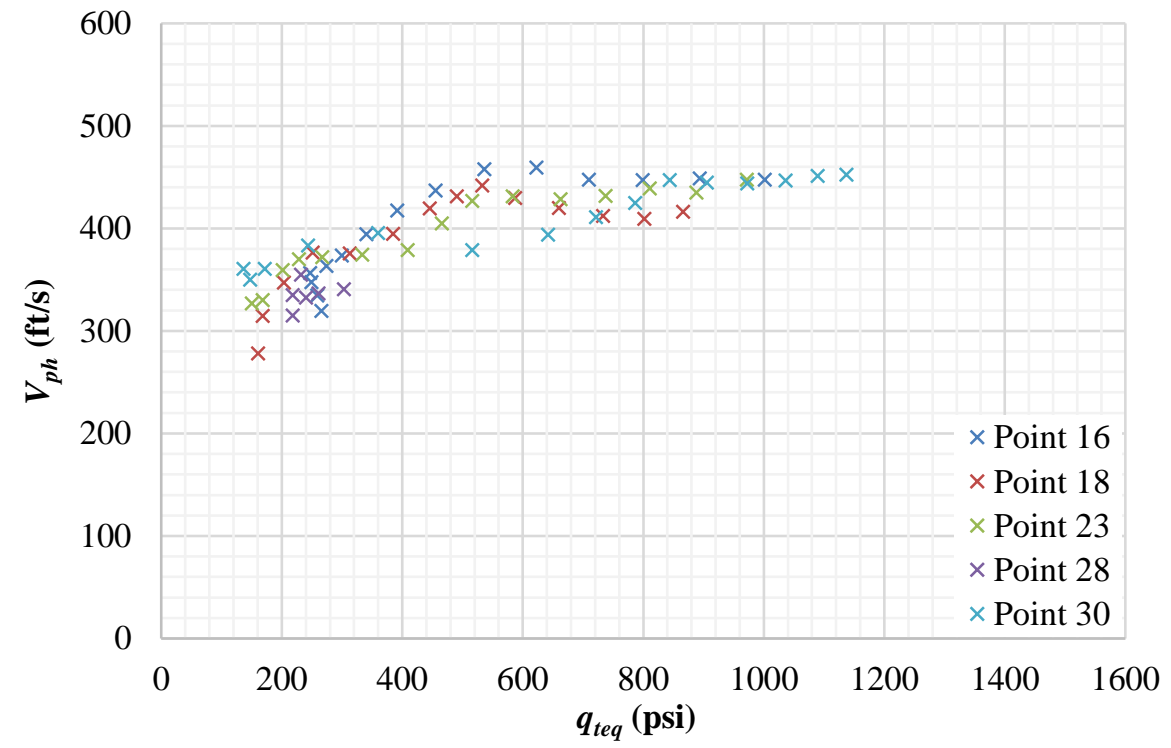

Figure B.4 - Group 23 equivalent tip resistance $\left(q_{t e q}\right)$ vs phase velocity $\left(V_{p h}\right)$ for the same $\lambda$. 


\section{Group 80}

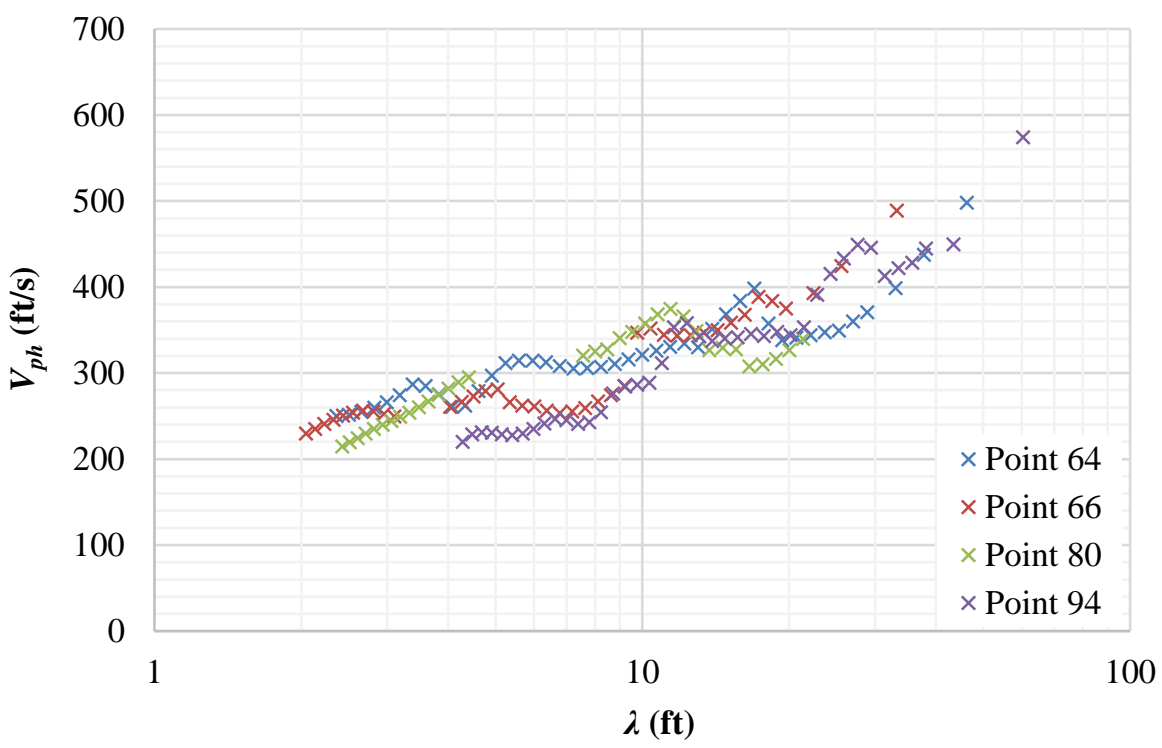

Figure B.5 - Group 80 dispersion curves.

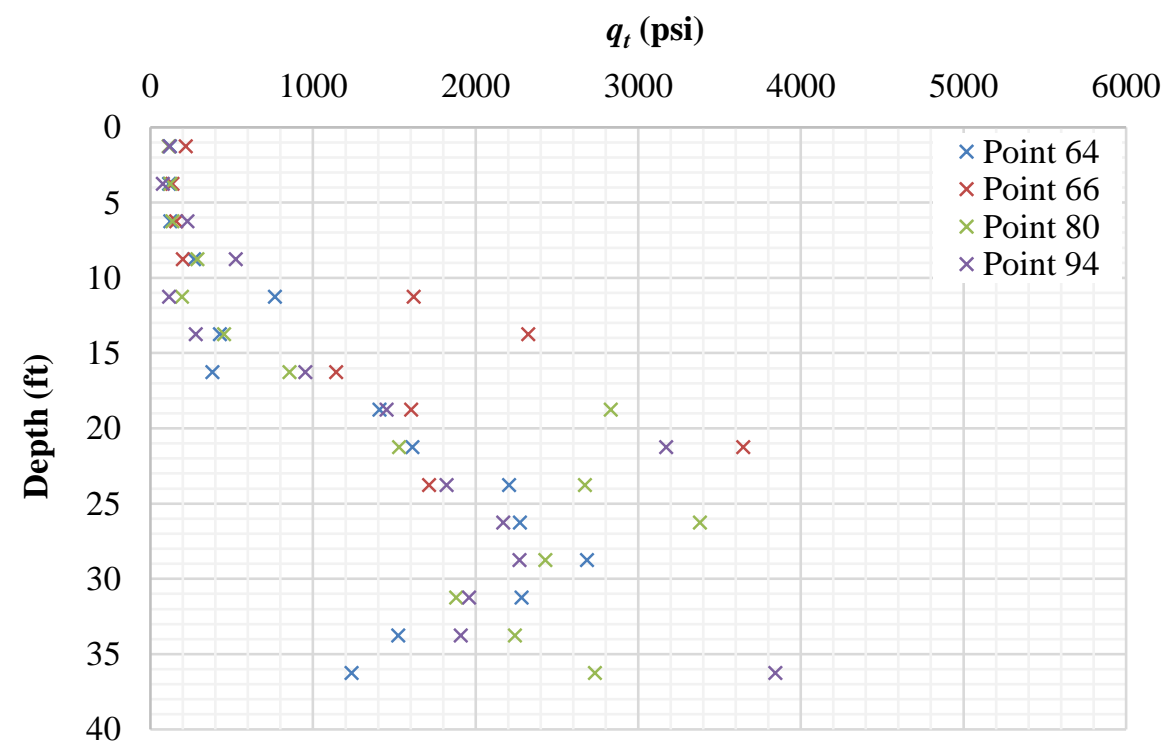

Figure B.6 - Group 80 tip resistance $\left(q_{t}\right)$ vs depth $(z)$. 


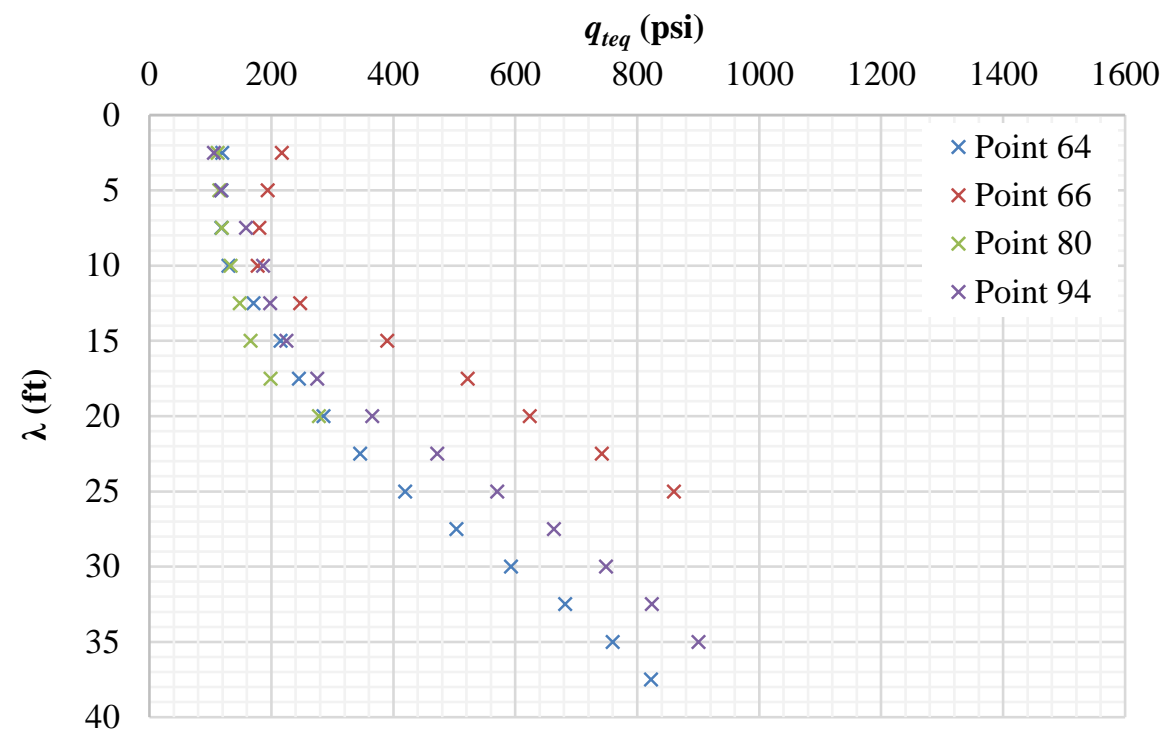

Figure B.7 - Group 80 equivalent tip resistance $\left(q_{t e q}\right)$ vs wavelength (ג).

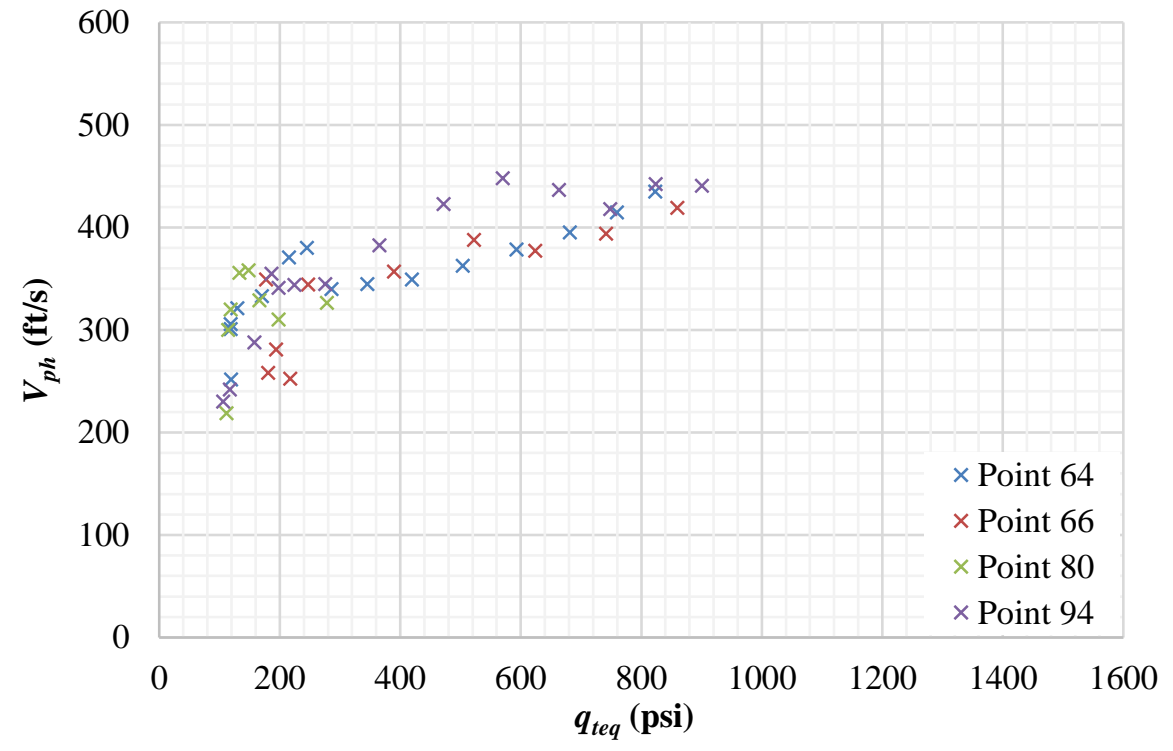

Figure B.8 - Group 80 equivalent tip resistance $\left(q_{t e q}\right)$ vs phase velocity $\left(V_{p h}\right)$ for the same $\lambda$. 


\section{Group 84}

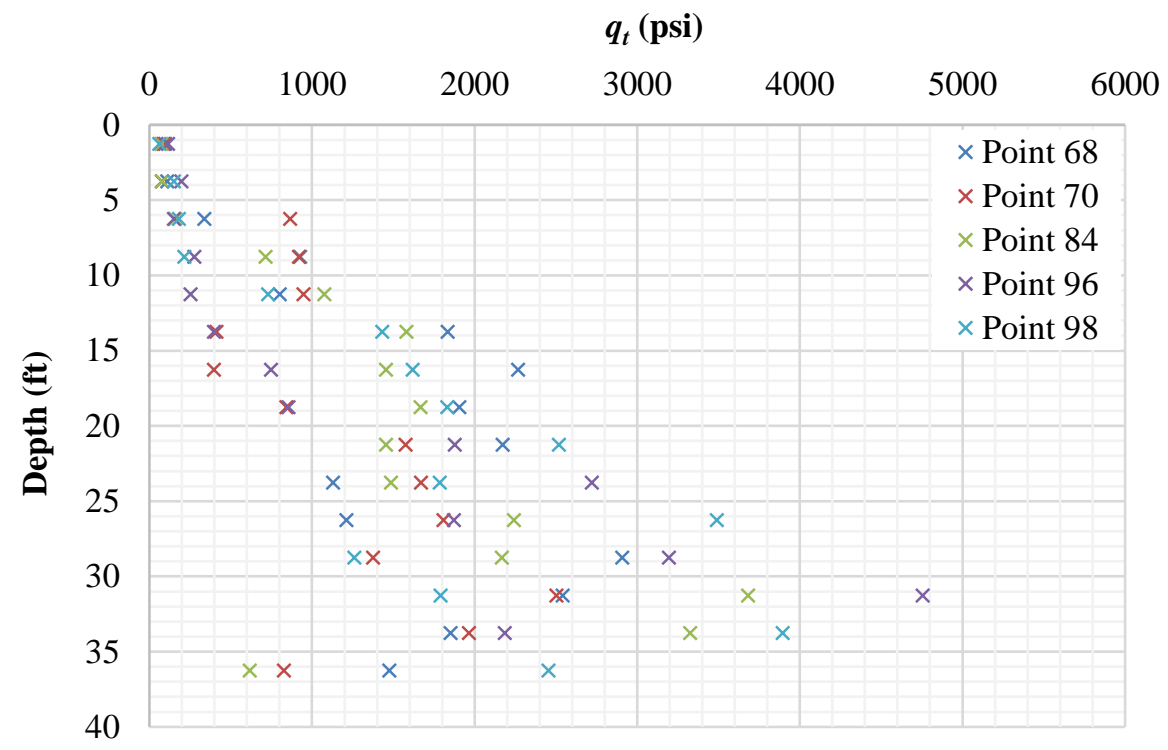

Figure B.9 - Group 84 tip resistance $\left(q_{t}\right)$ vs depth $(z)$. 


\section{Group 135}

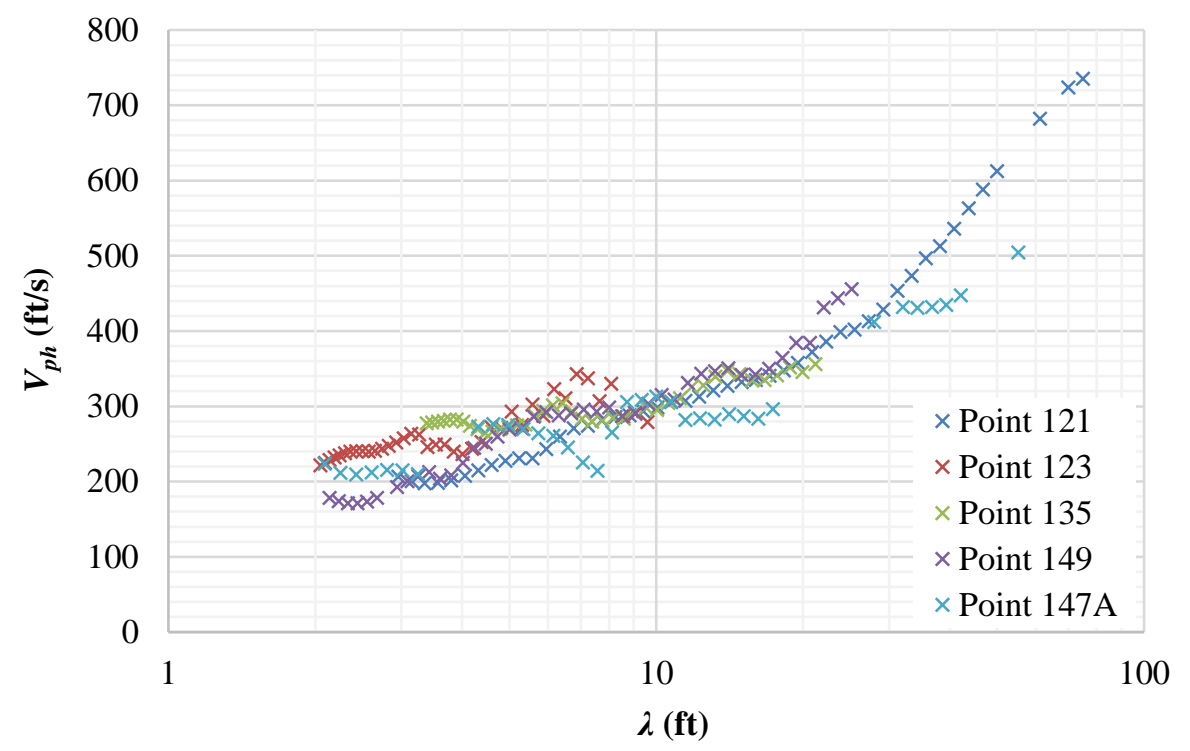

Figure B.10 - Group 135 dispersion curves.

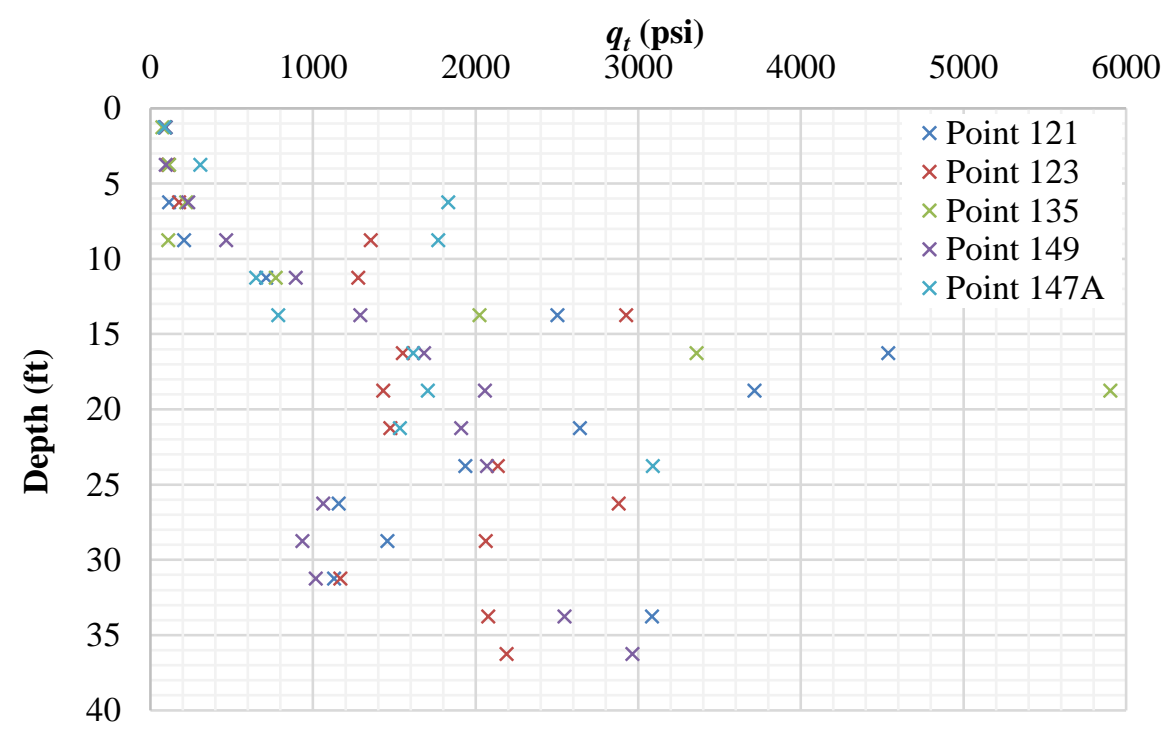

Figure B.11 - Group 135 tip resistance $\left(q_{t}\right)$ vs depth (z). 


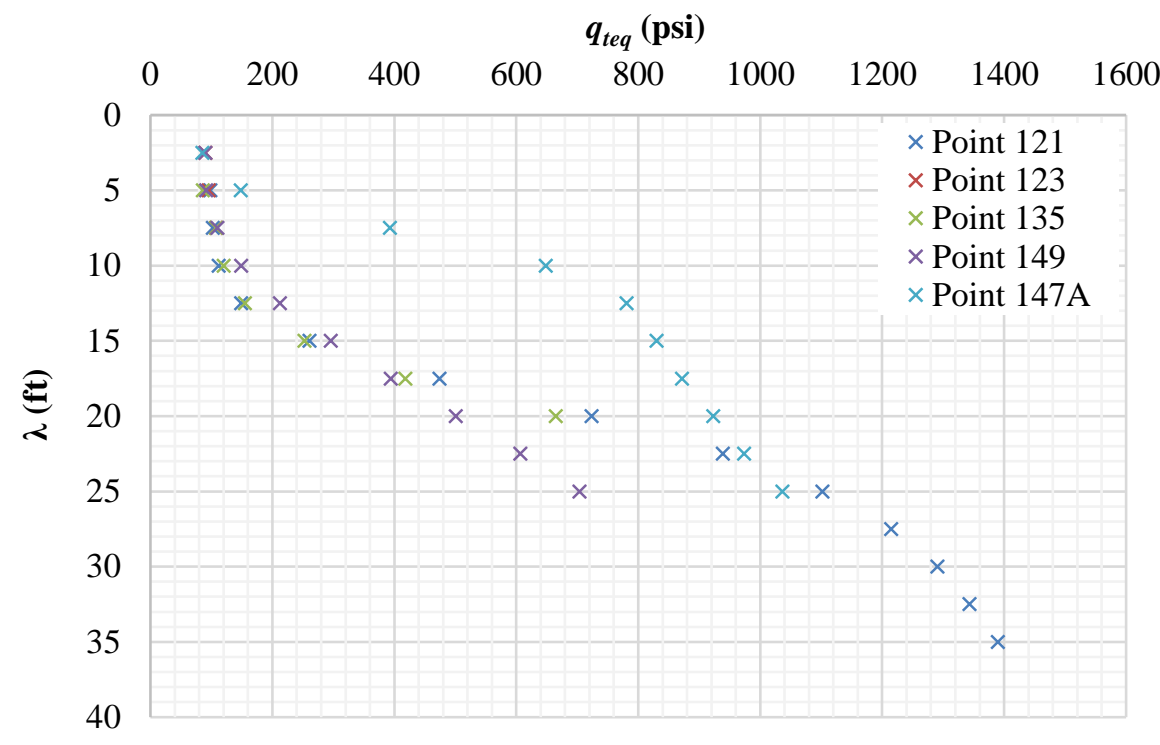

Figure B.12 - Group 135 equivalent tip resistance $\left(q_{t e q}\right)$ vs wavelength $(\lambda)$.

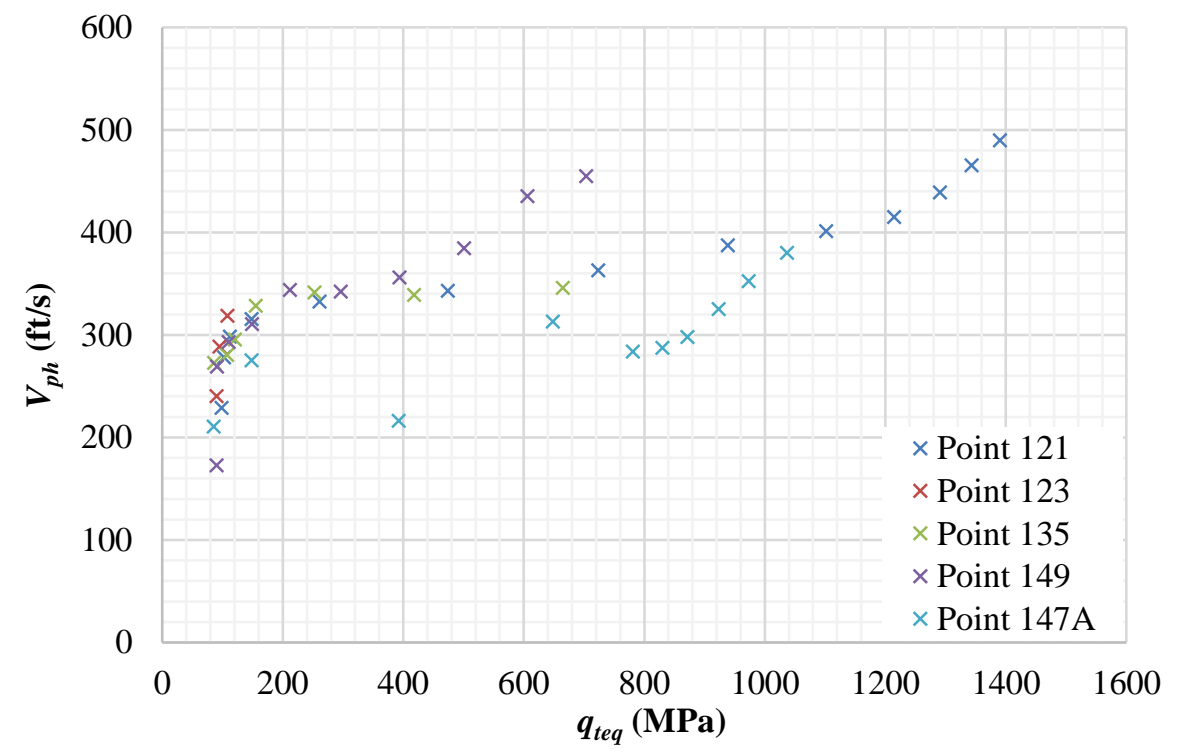

Figure B.13 - Group 135 equivalent tip resistance $\left(q_{t e q}\right)$ vs phase velocity $\left(V_{p h}\right)$ for the same $\lambda$. 


\section{Group 180}

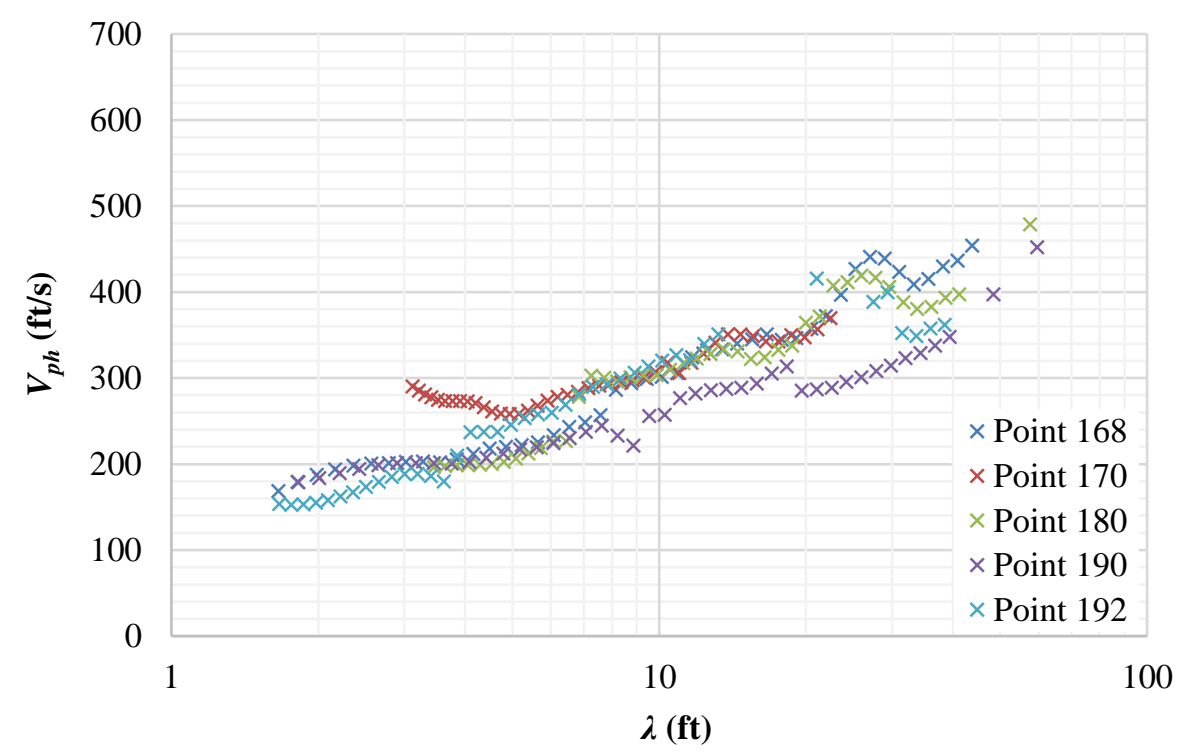

Figure B.14 - Group 180 dispersion curves.

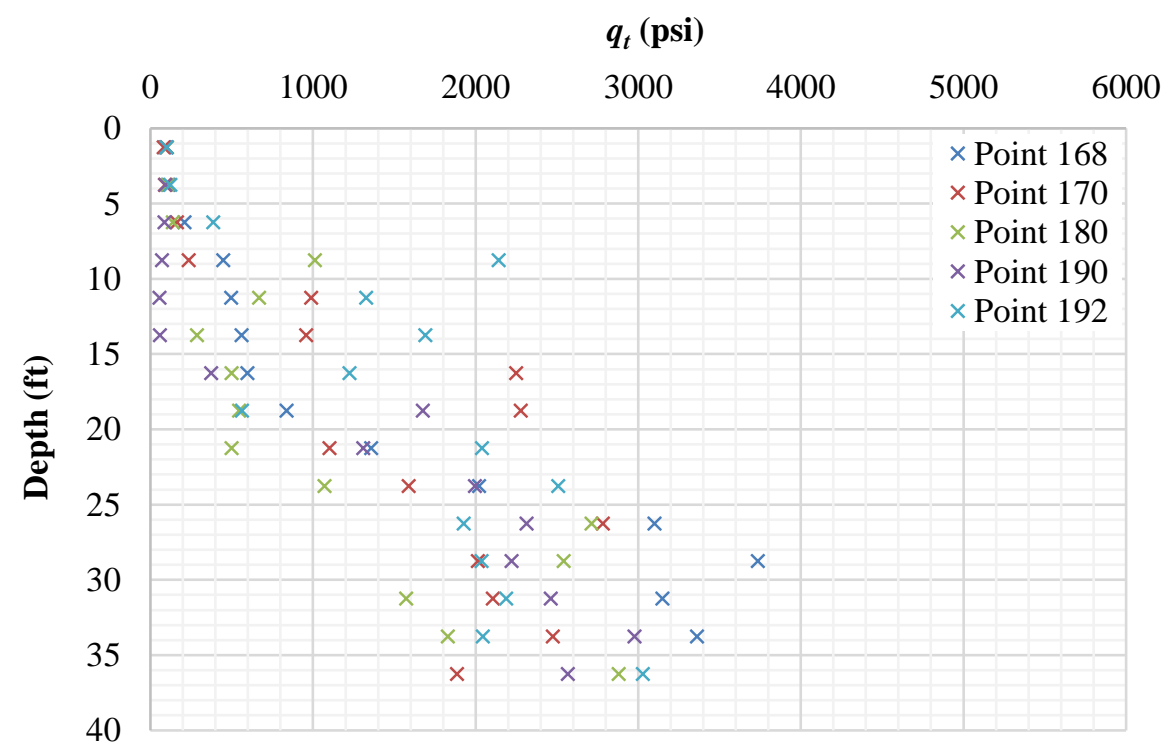

Figure B.15 - Group 180 tip resistance $\left(q_{t}\right)$ vs depth (z). 


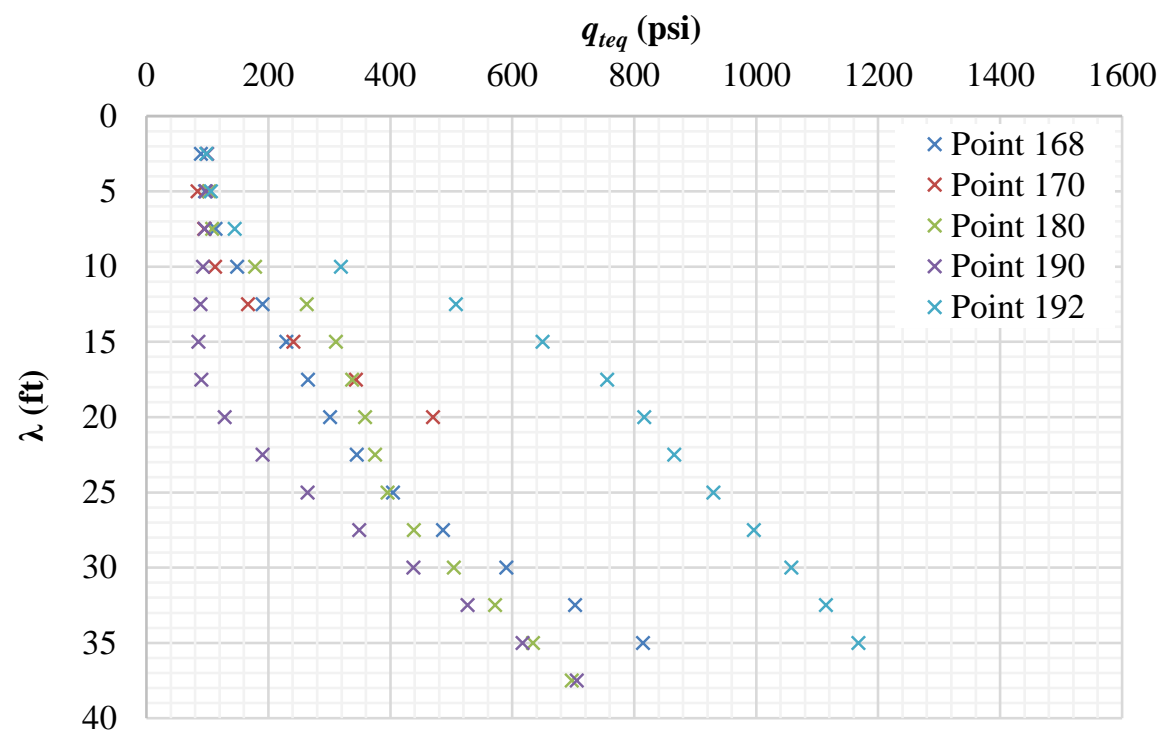

Figure B.16 - Group 180 equivalent tip resistance $\left(q_{t e q}\right)$ vs wavelength (

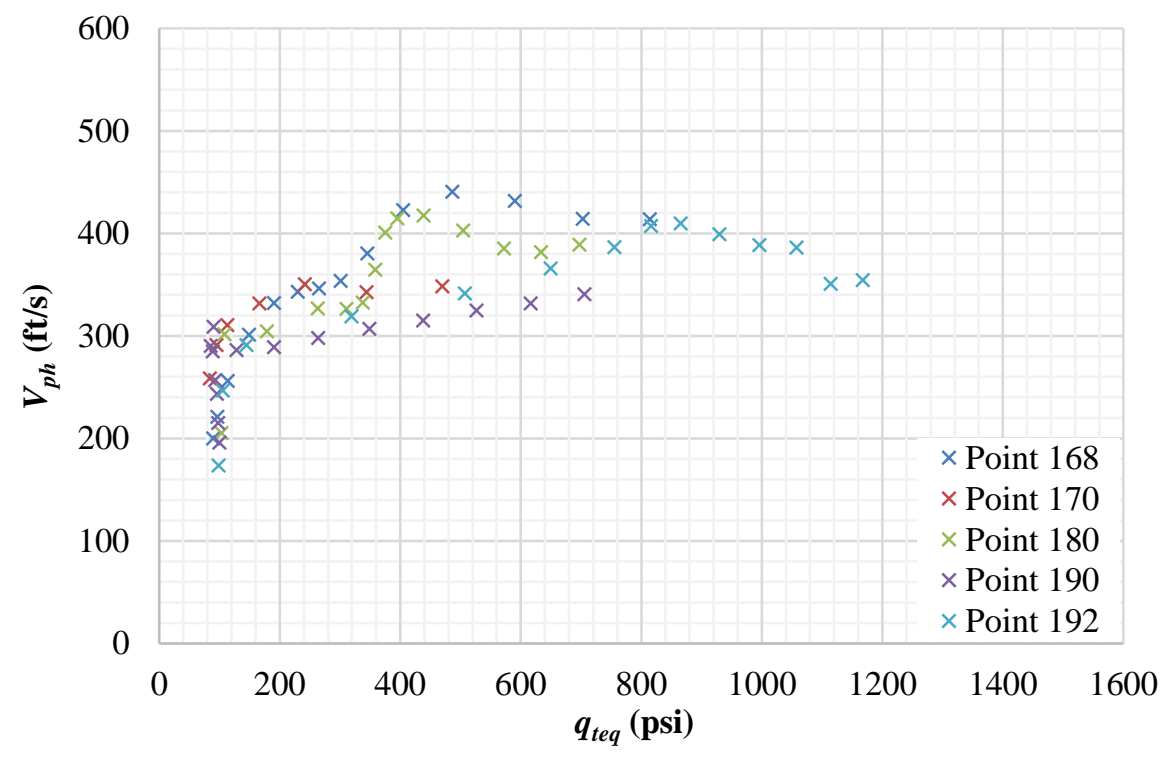

Figure B.17 - Group 180 equivalent tip resistance $\left(q_{t e q}\right)$ vs phase velocity $\left(V_{p h}\right)$ for the same $\lambda$. 


\section{Group 184}

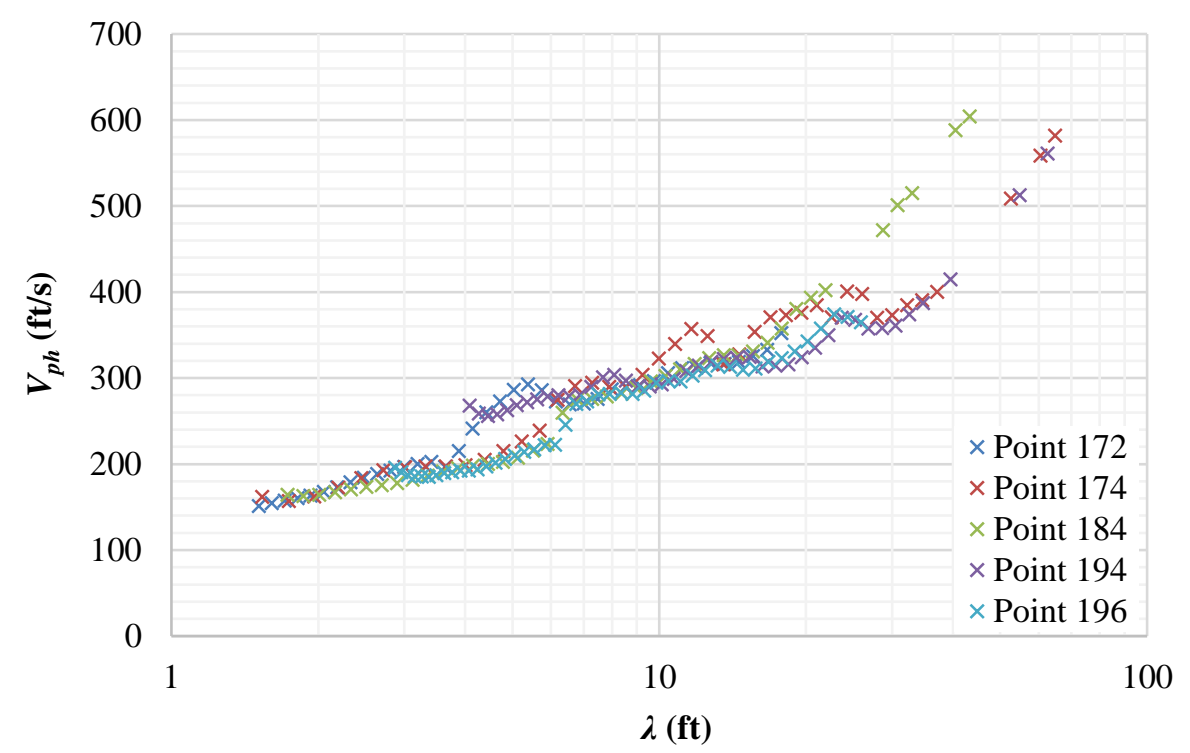

Figure B.18 - Group 184 dispersion curves.

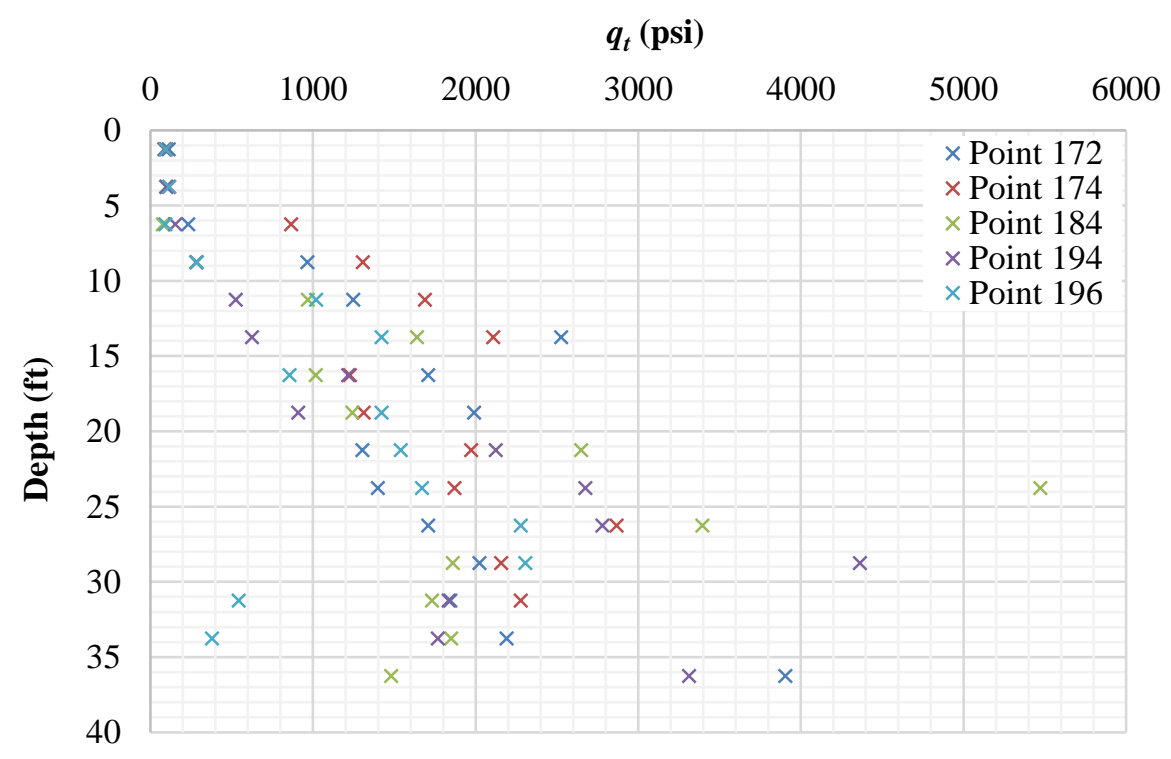

Figure B.19 - Group 184 tip resistance $\left(q_{t}\right)$ vs depth (z). 


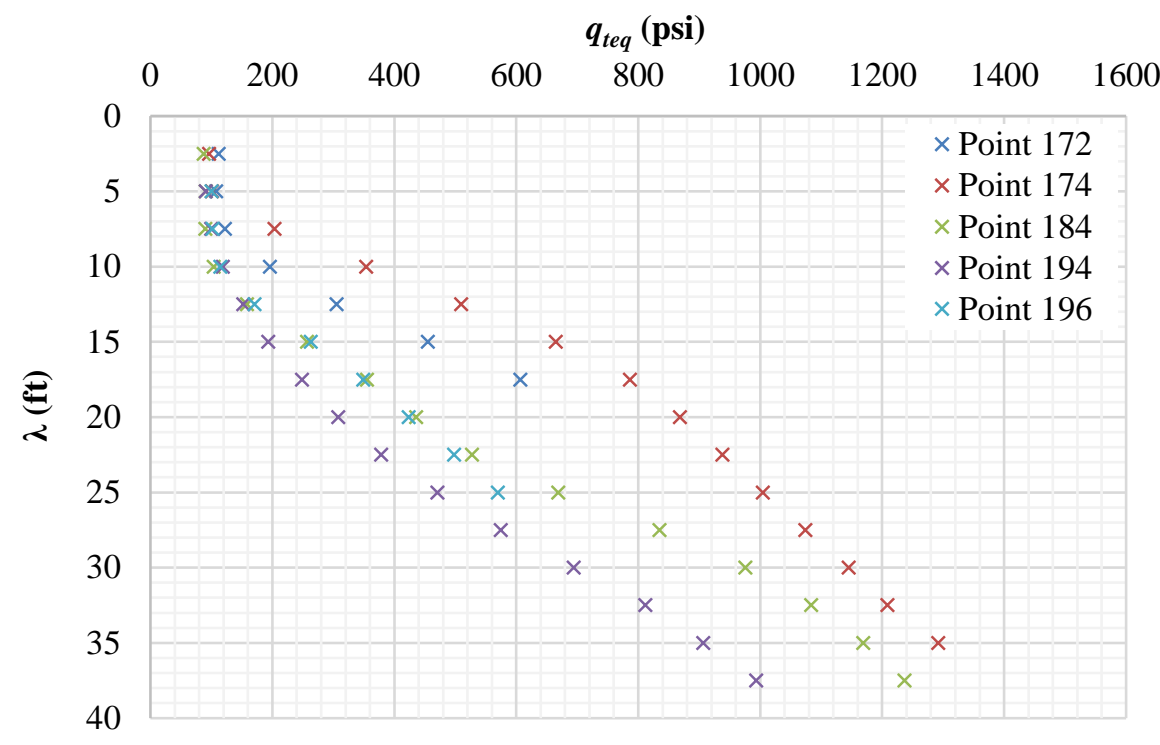

Figure B.20 - Group 184 equivalent tip resistance $\left(q_{t e q}\right)$ vs wavelength $(\lambda)$.

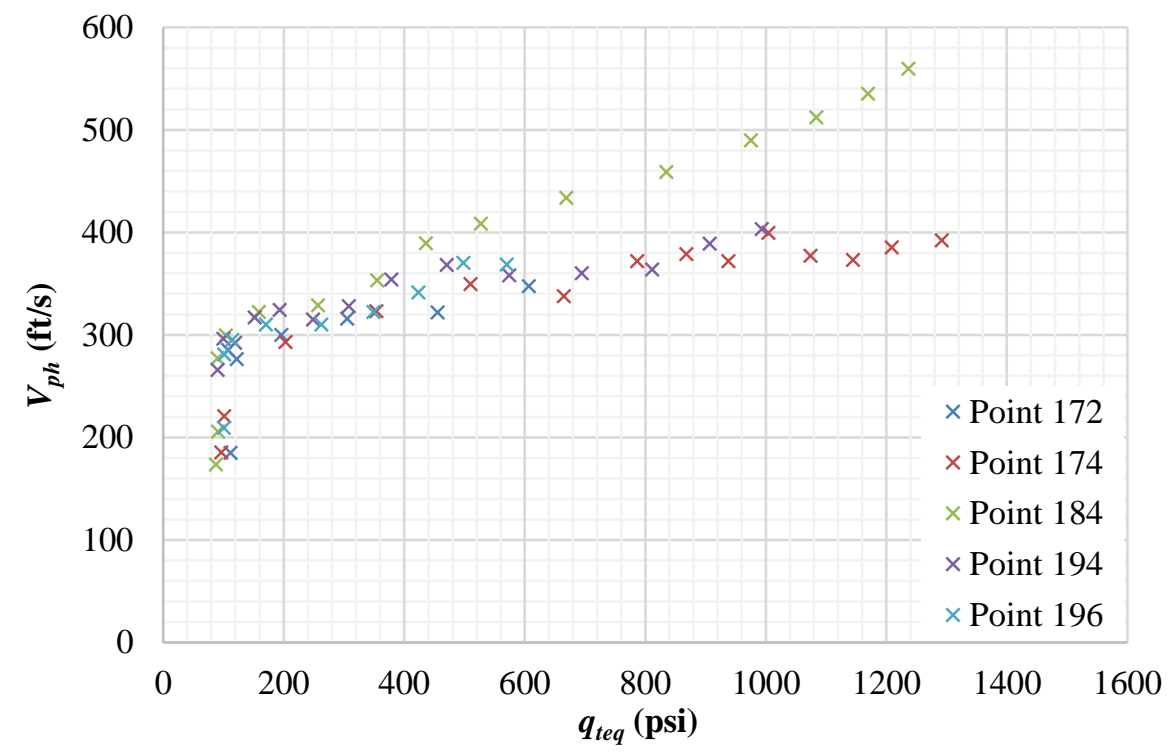

Figure B.21 - Group 184 equivalent tip resistance $\left(q_{\text {teq }}\right)$ vs phase velocity $\left(V_{p h}\right)$ for the same $\lambda$. 


\section{Appendix C: Measured vs calculated dispersion curves}

$$
V_{p h}(f t / s)=101\left(q_{t e q}(p s i)\right)^{0.212}
$$

\section{Group 23}

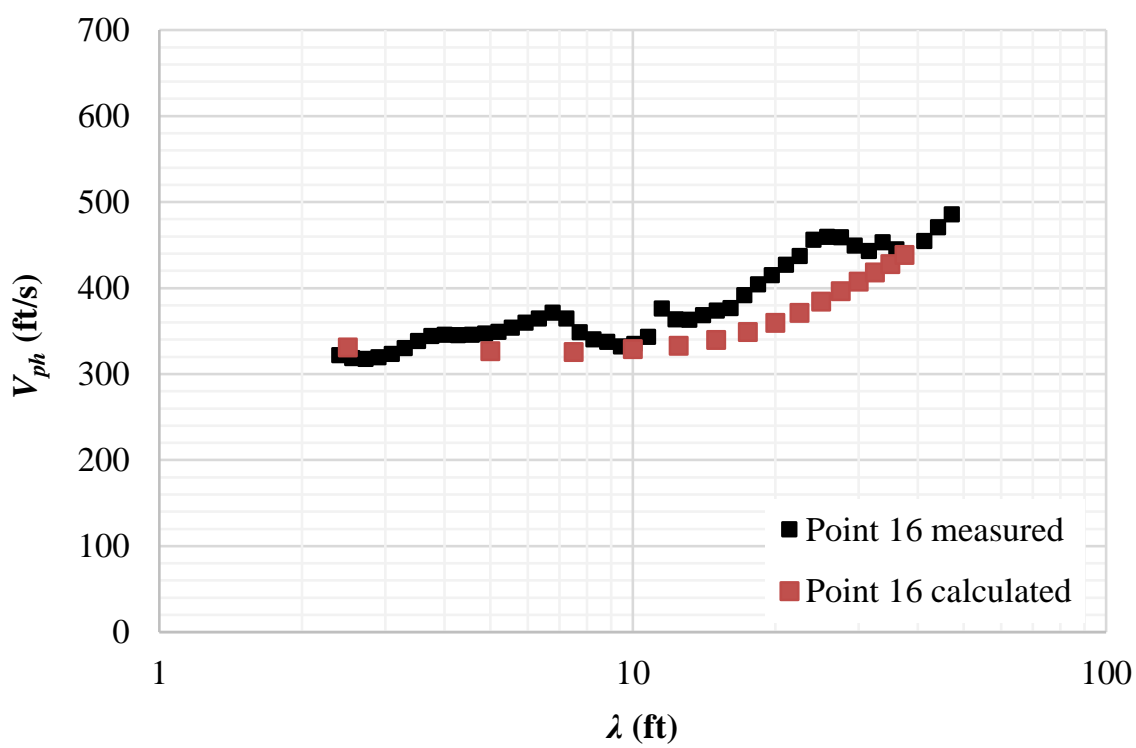

Figure C.1 - Point 16 measured vs calculated dispersion curves.

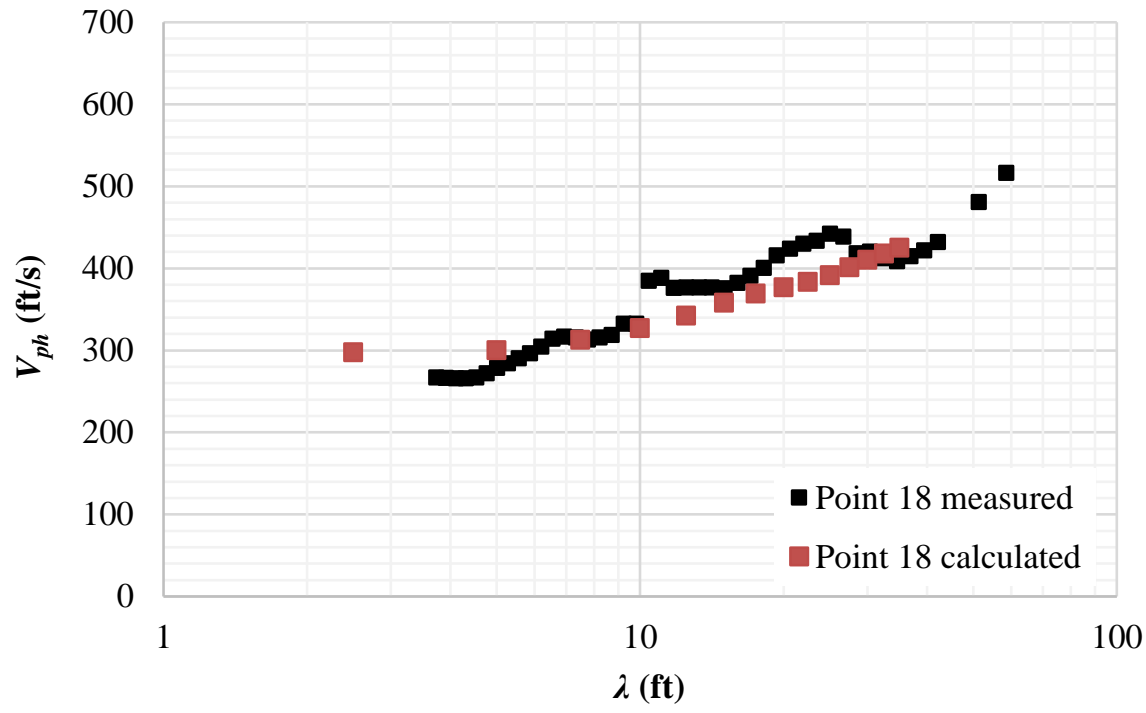

Figure C.2 - Point 18 measured vs calculated dispersion curves. 


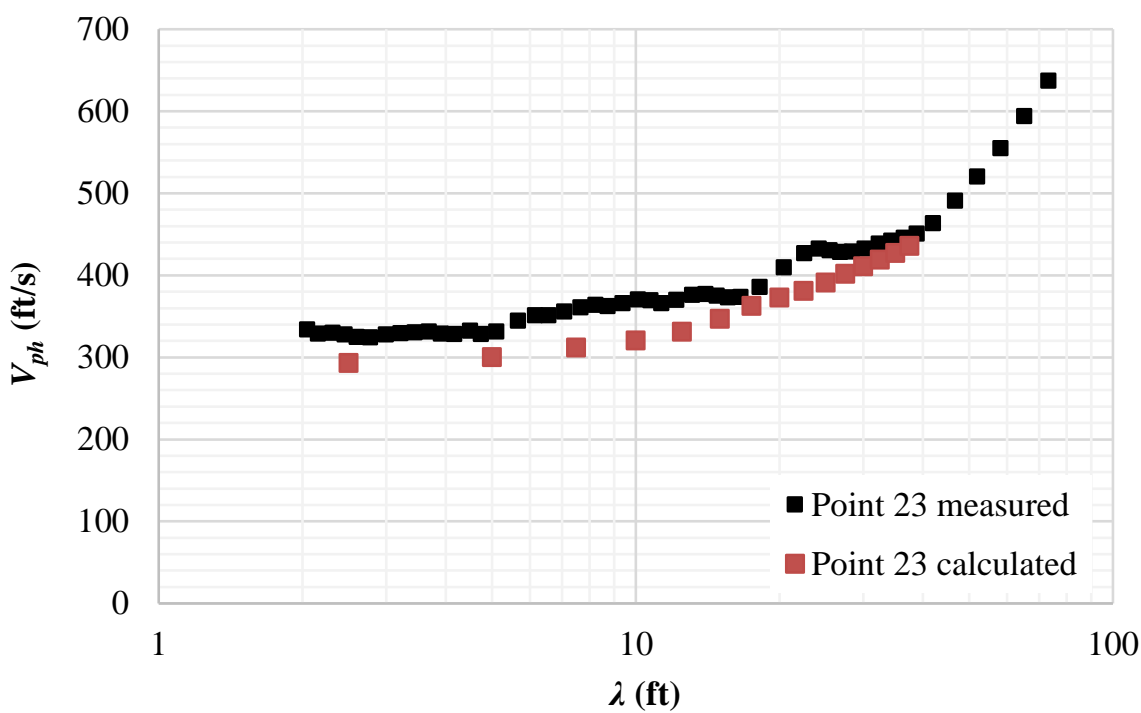

Figure C.3 - Point 23 measured vs calculated dispersion curves.

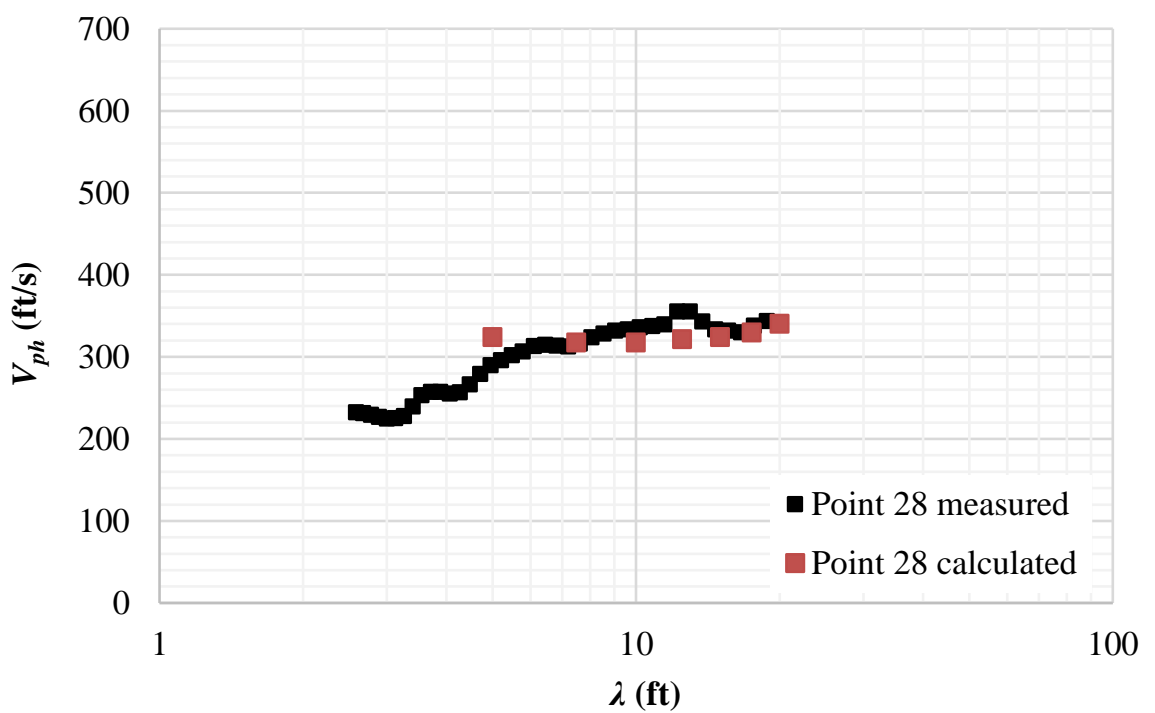

Figure C.4 - Point 28 measured vs calculated dispersion curves. 


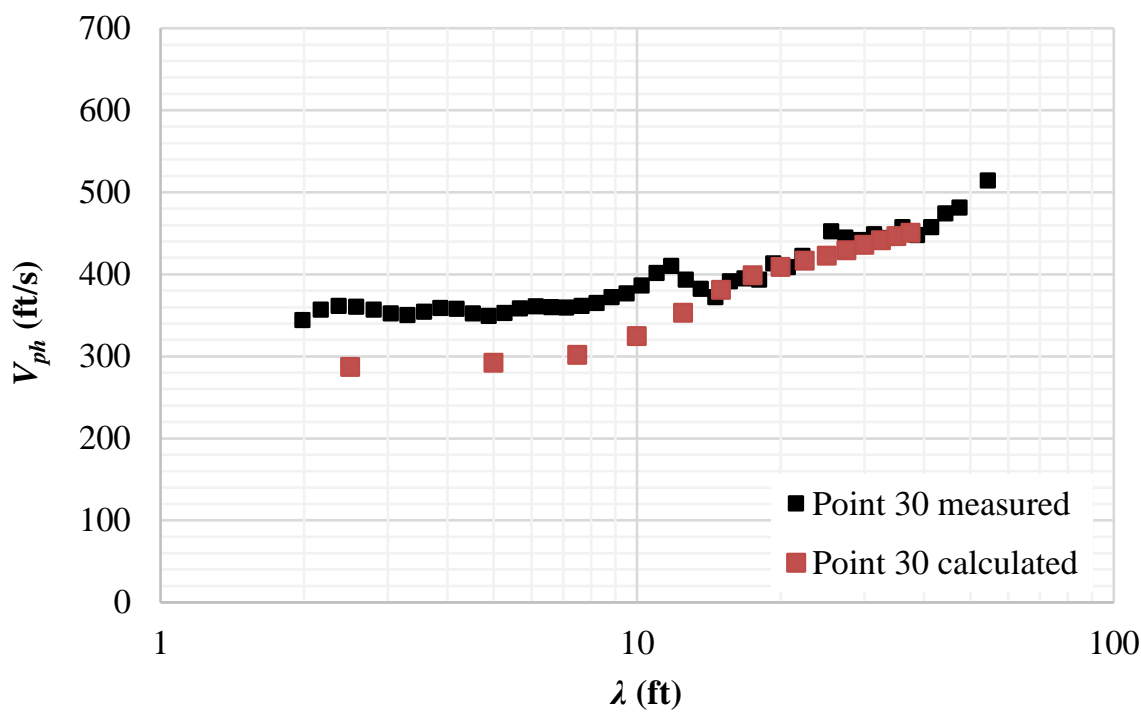

Figure C.5 - Point 30 measured vs calculated dispersion curves.

\section{Group 80}

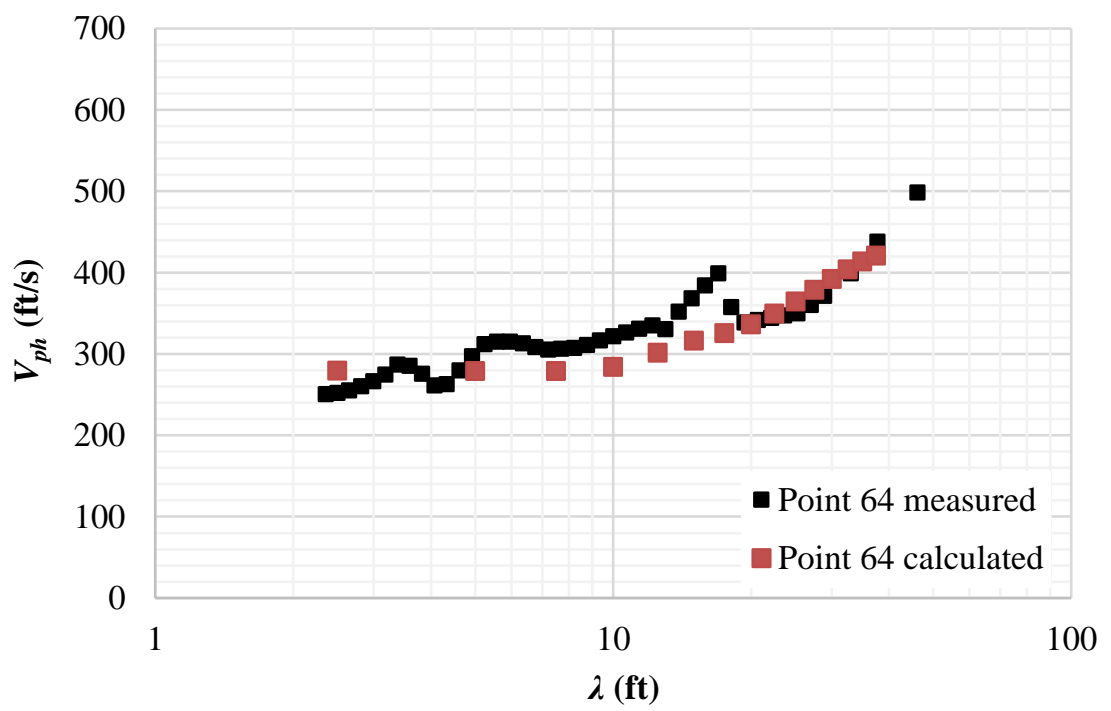

Figure C.6 - Point 64 measured vs calculated dispersion curves. 


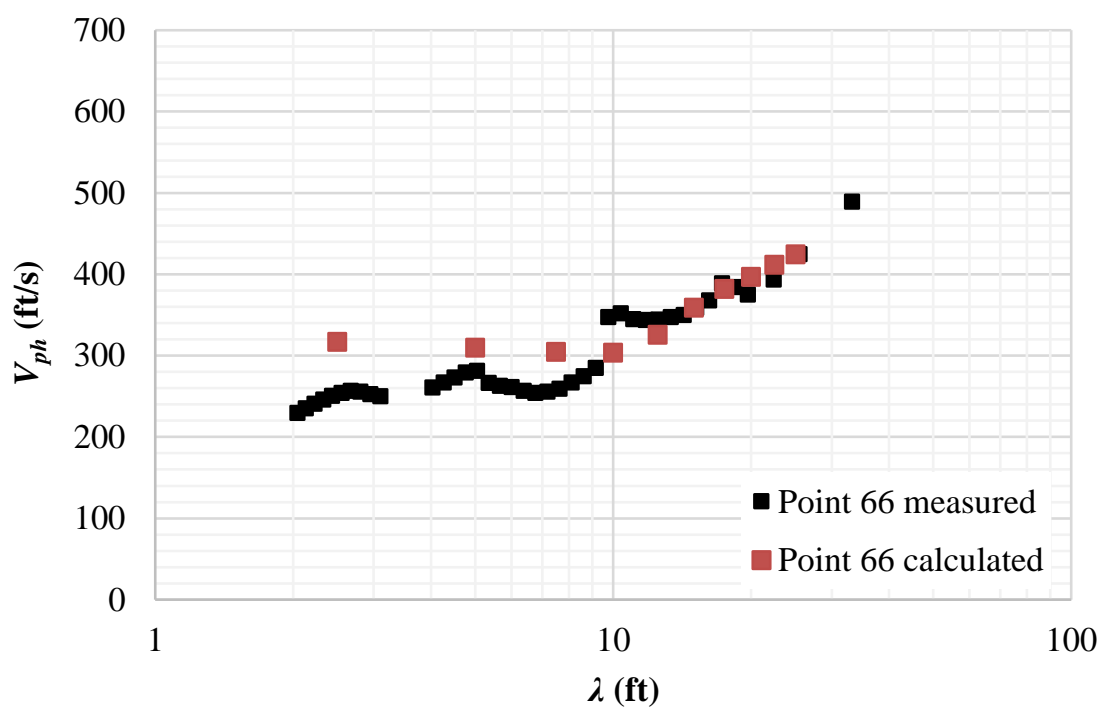

Figure C.7 - Point 66 measured vs calculated dispersion curves.

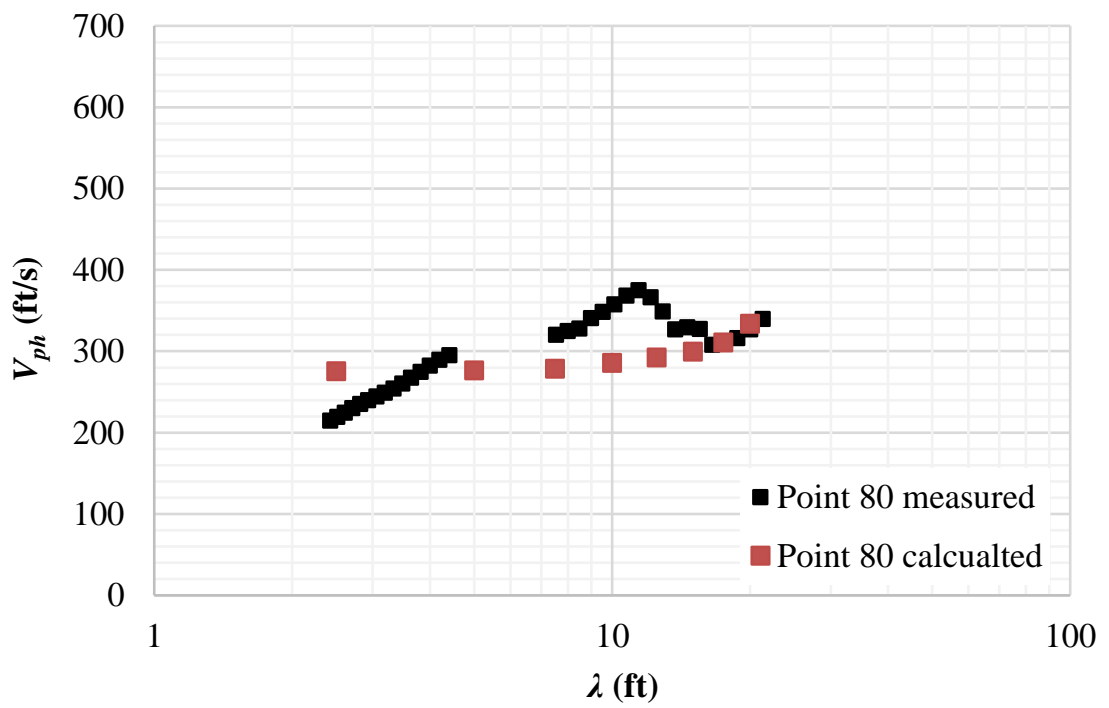

Figure C.8 - Point 80 measured vs calculated dispersion curves. 


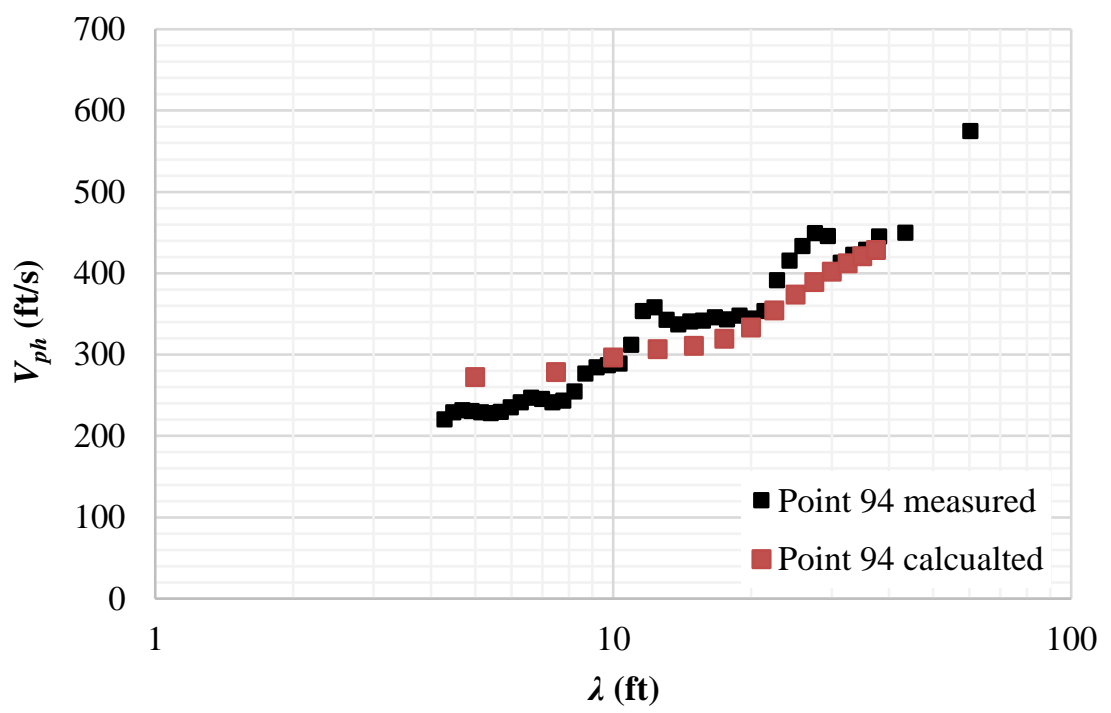

Figure C.9 - Point 94 measured vs calculated dispersion curves.

\section{Group 135}

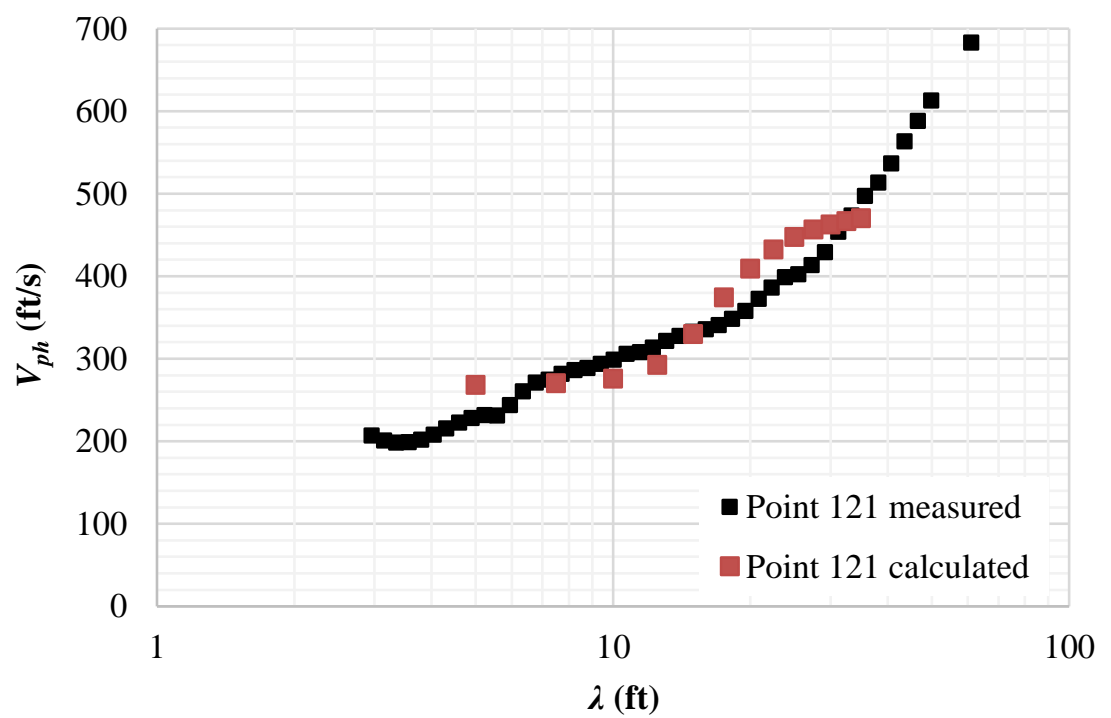

Figure C.10 - Point 121 measured vs calculated dispersion curves. 


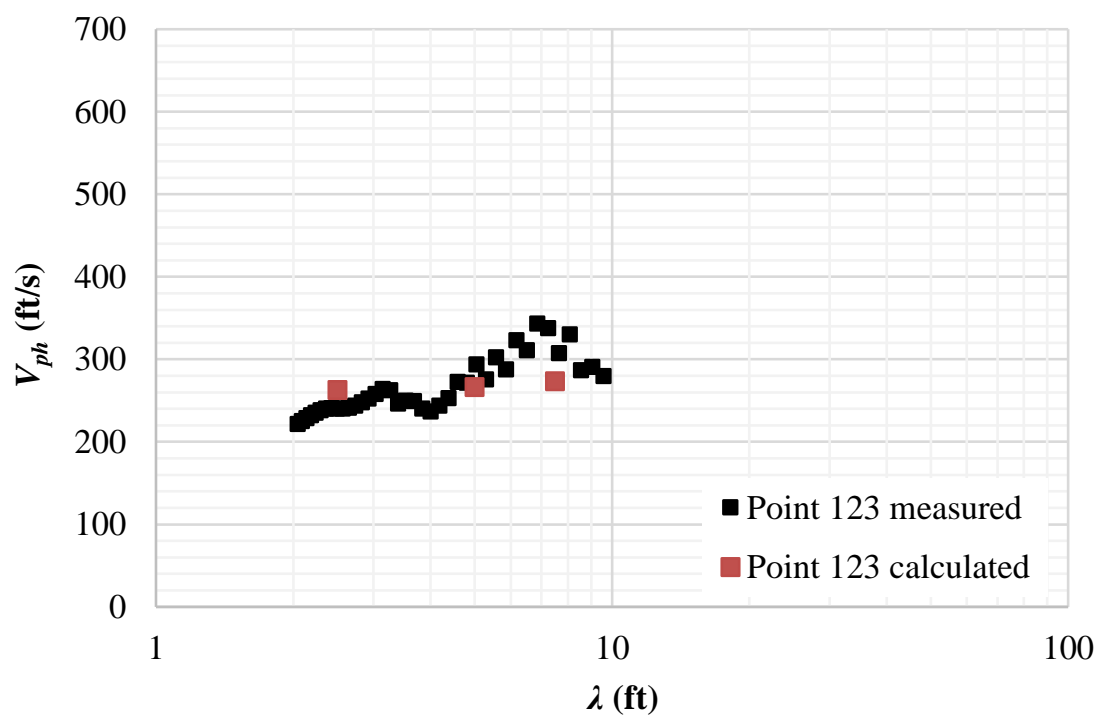

Figure C.11 - Point 123 measured vs calculated dispersion curves.

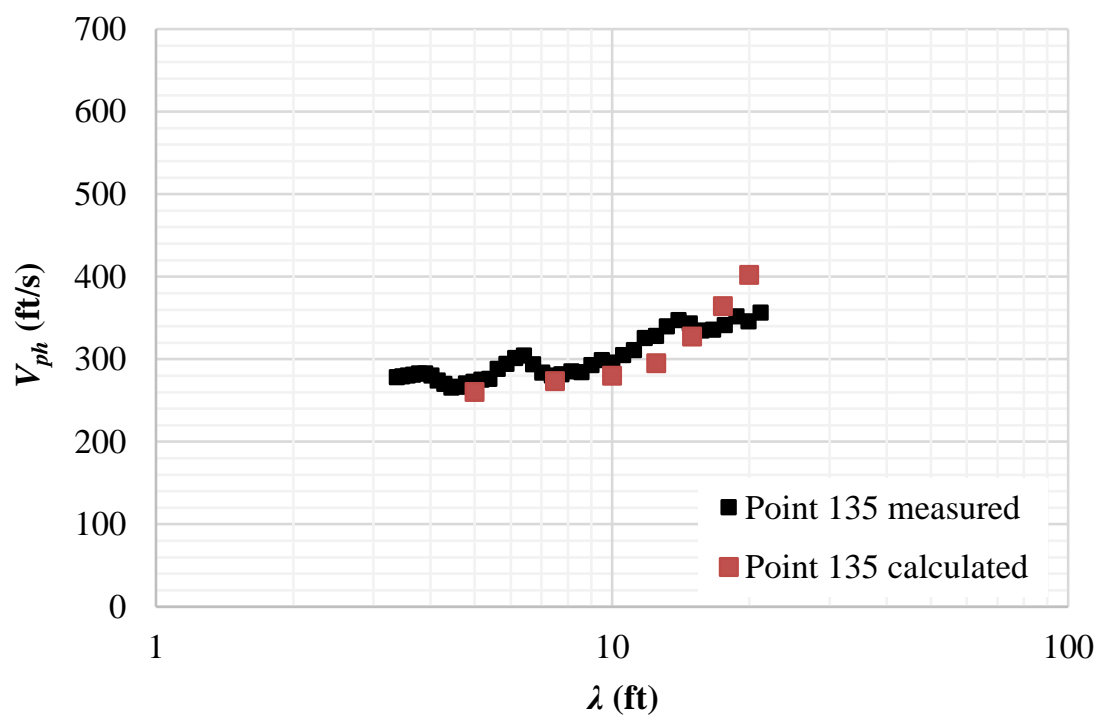

Figure C.12 - Point 135 measured vs calculated dispersion curves. 


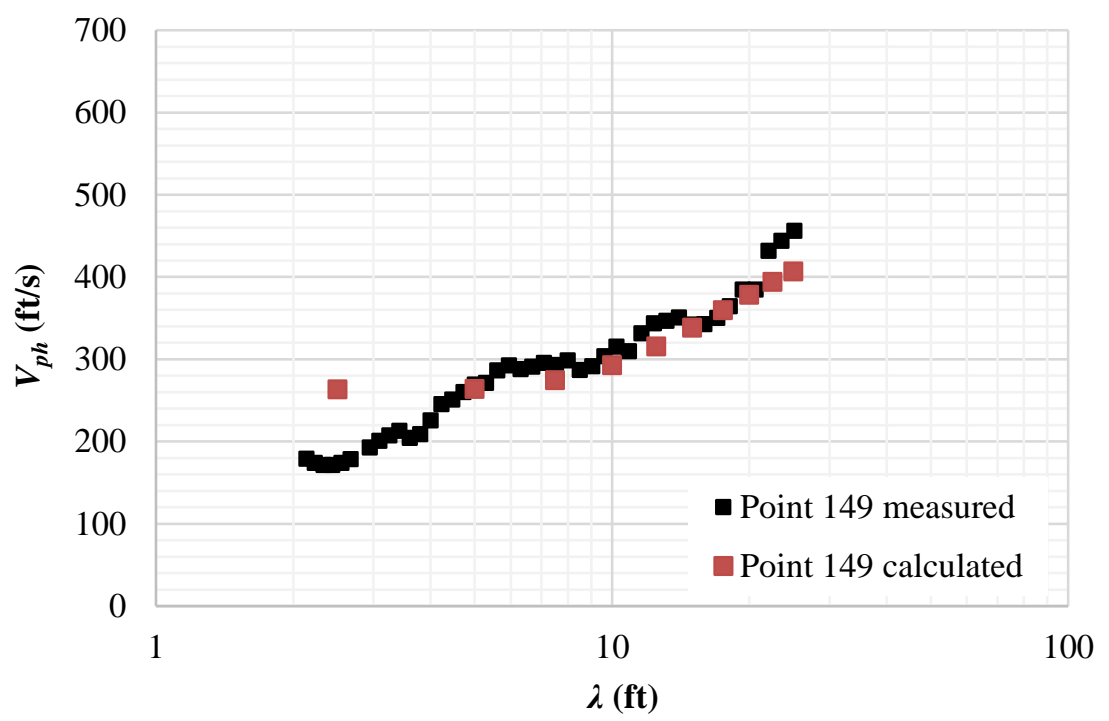

Figure C.13 - Point 149 measured vs calculated dispersion curves.

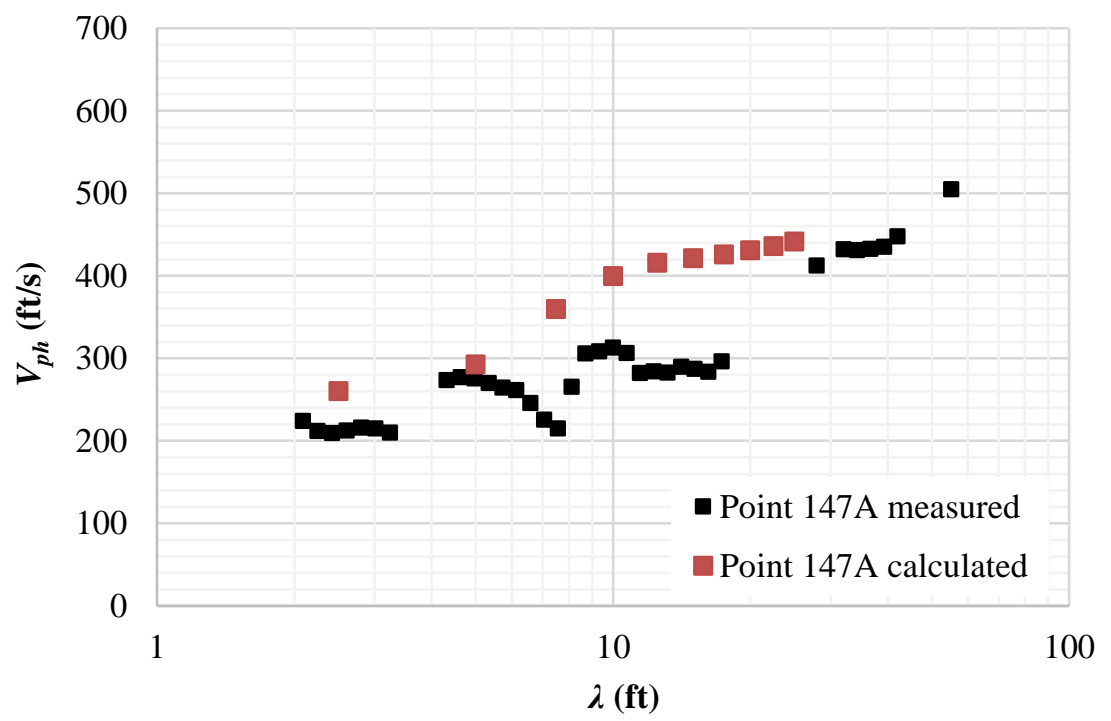

Figure C.14 - Point 147A measured vs calculated dispersion curves. 


\section{Group 180}

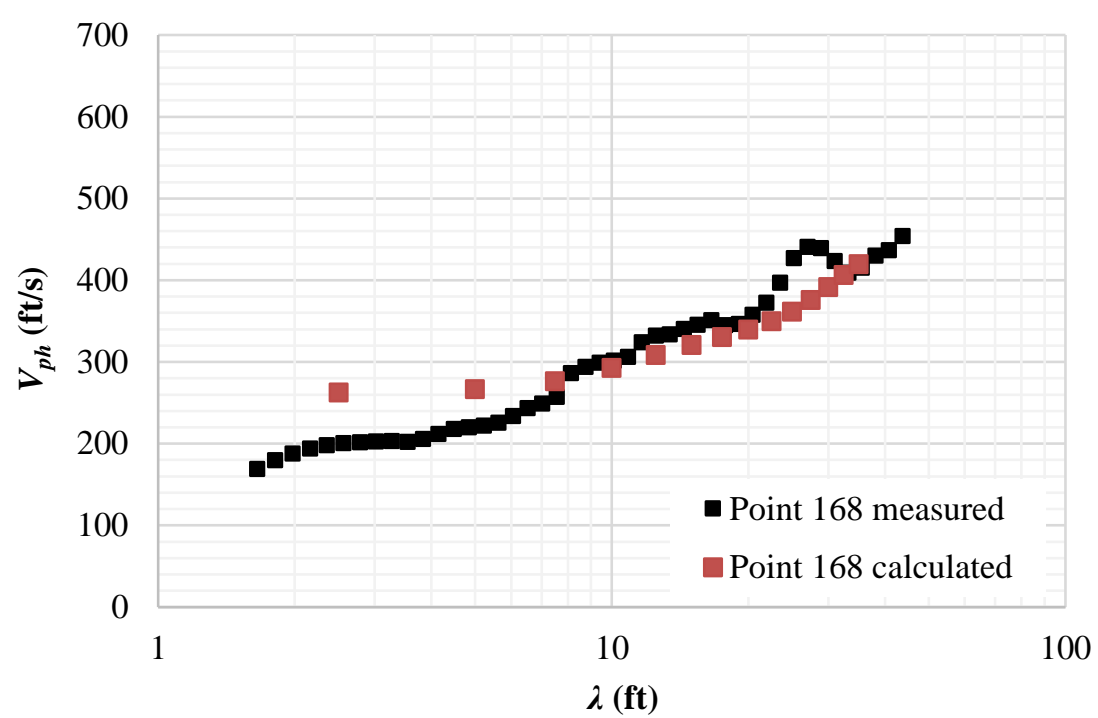

Figure C.15 - Point 168 measured vs calculated dispersion curves.

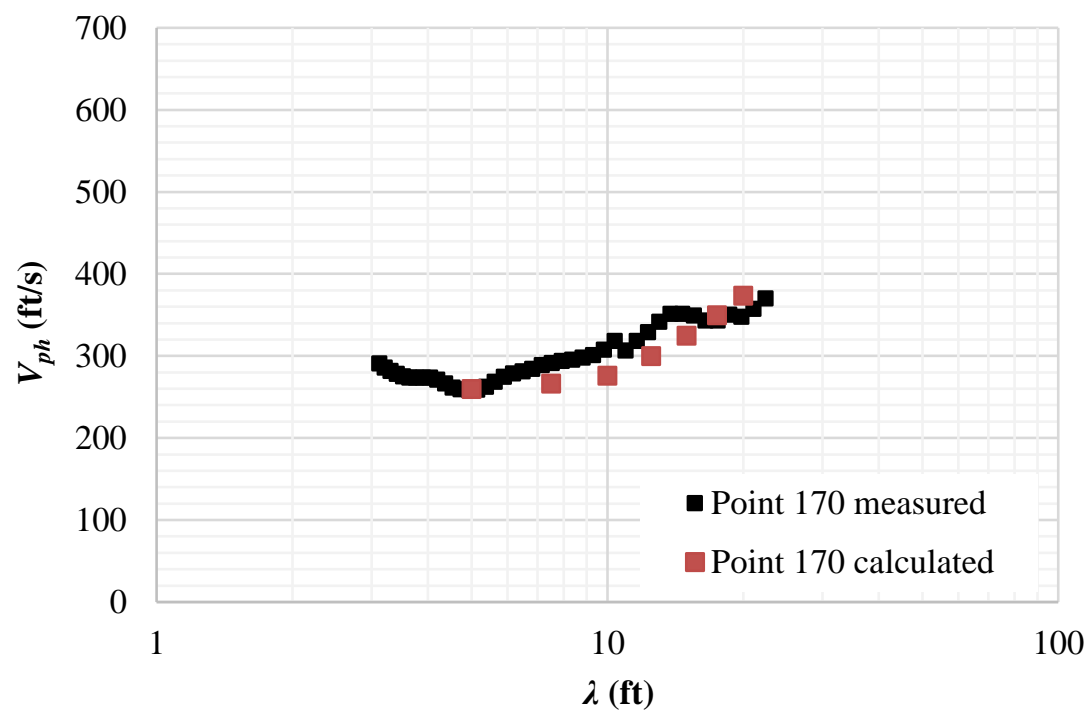

Figure C.16 - Point 170 measured vs calculated dispersion curves. 


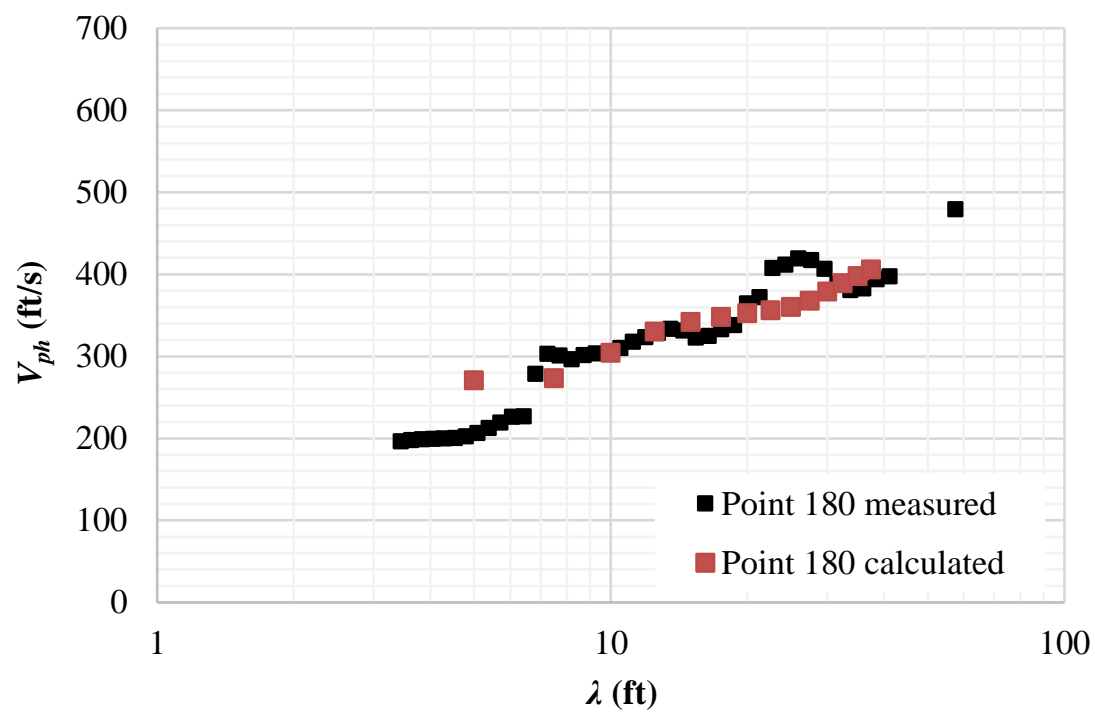

Figure C.17 - Point 180 measured vs calculated dispersion curves.

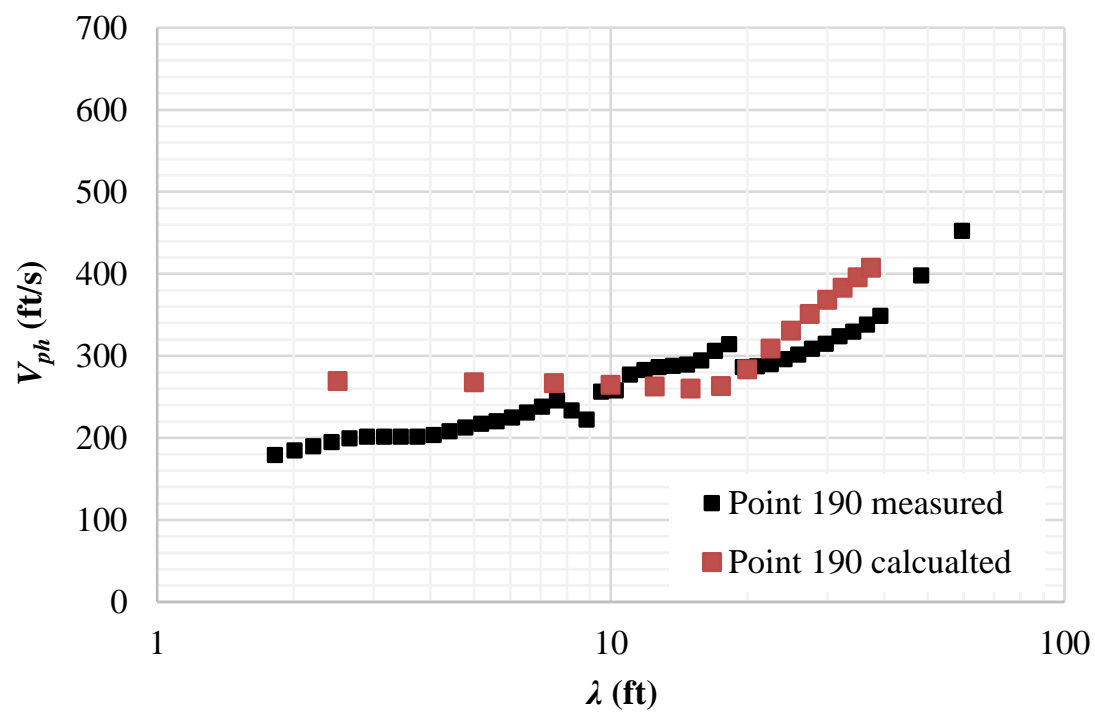

Figure C.18 - Point 190 measured vs calculated dispersion curves. 


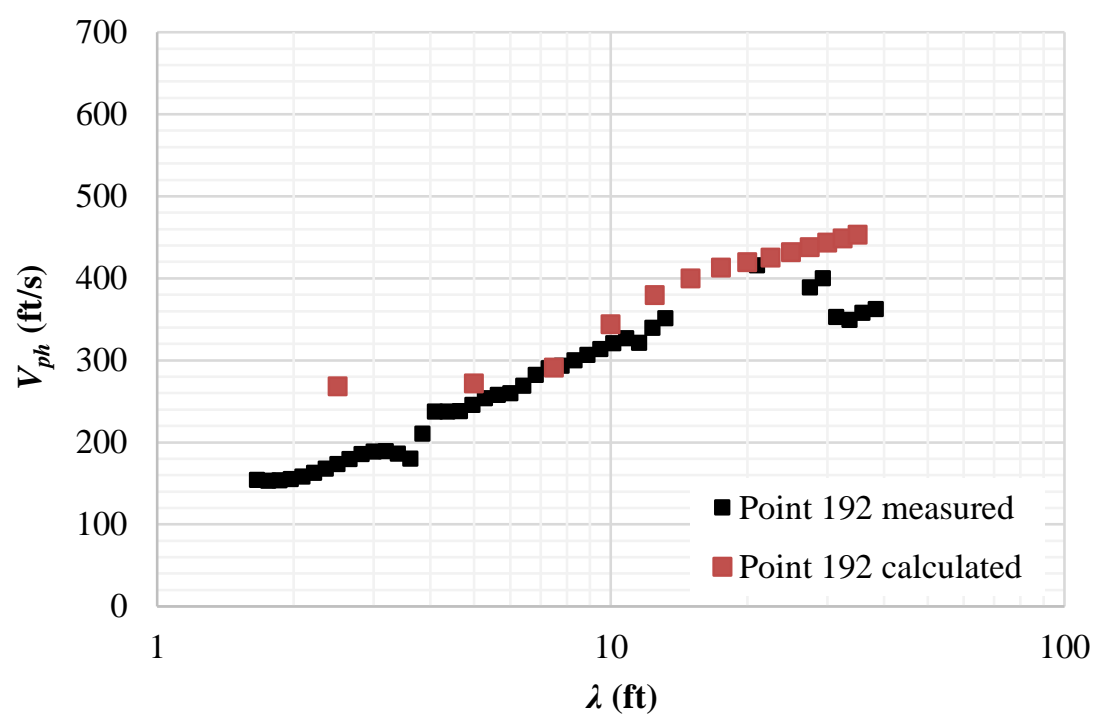

Figure C.19 - Point 192 measured vs calculated dispersion curves.

\section{Group 184}

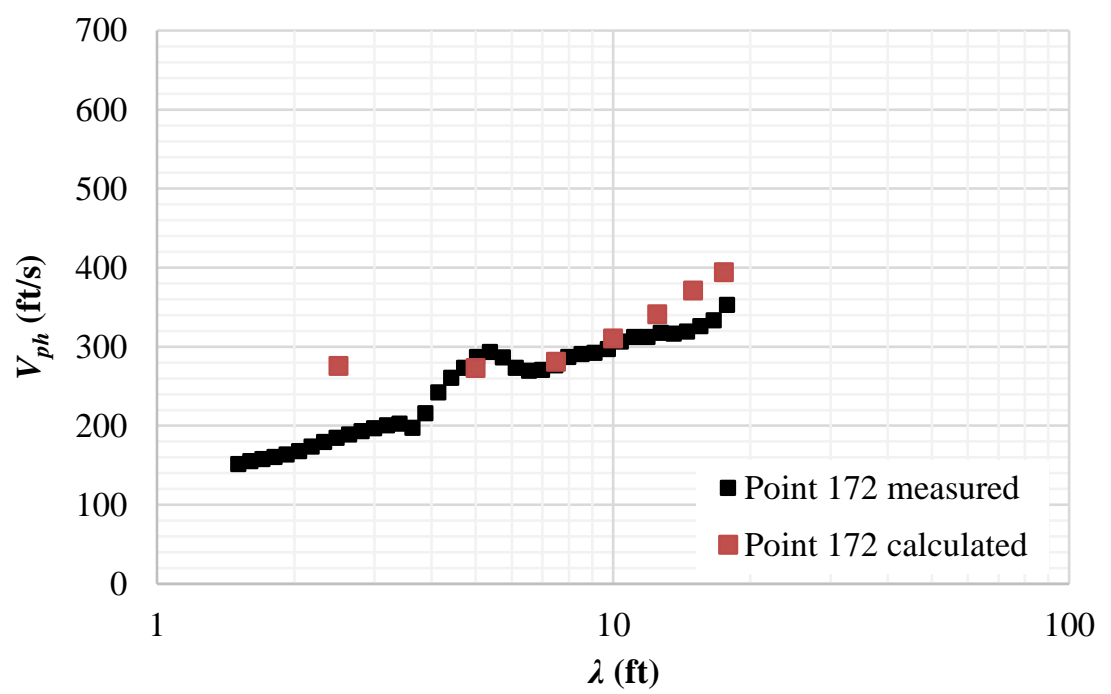

Figure C.20 - Point 172 measured vs calculated dispersion curves. 


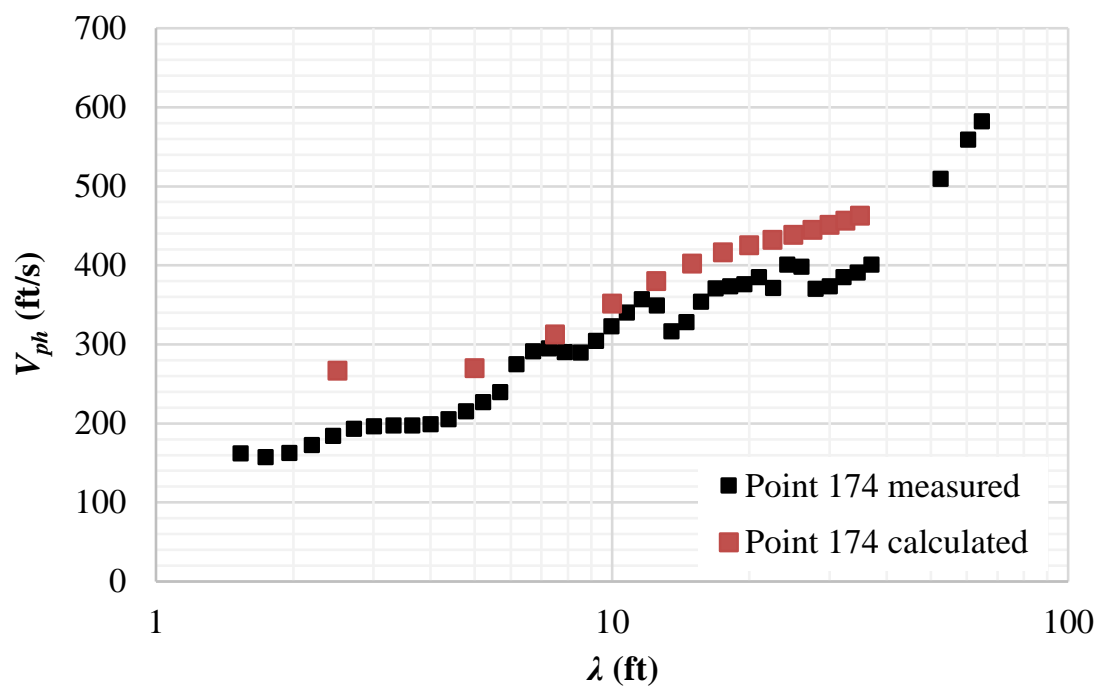

Figure C.21 - Point 174 measured vs calculated dispersion curves.

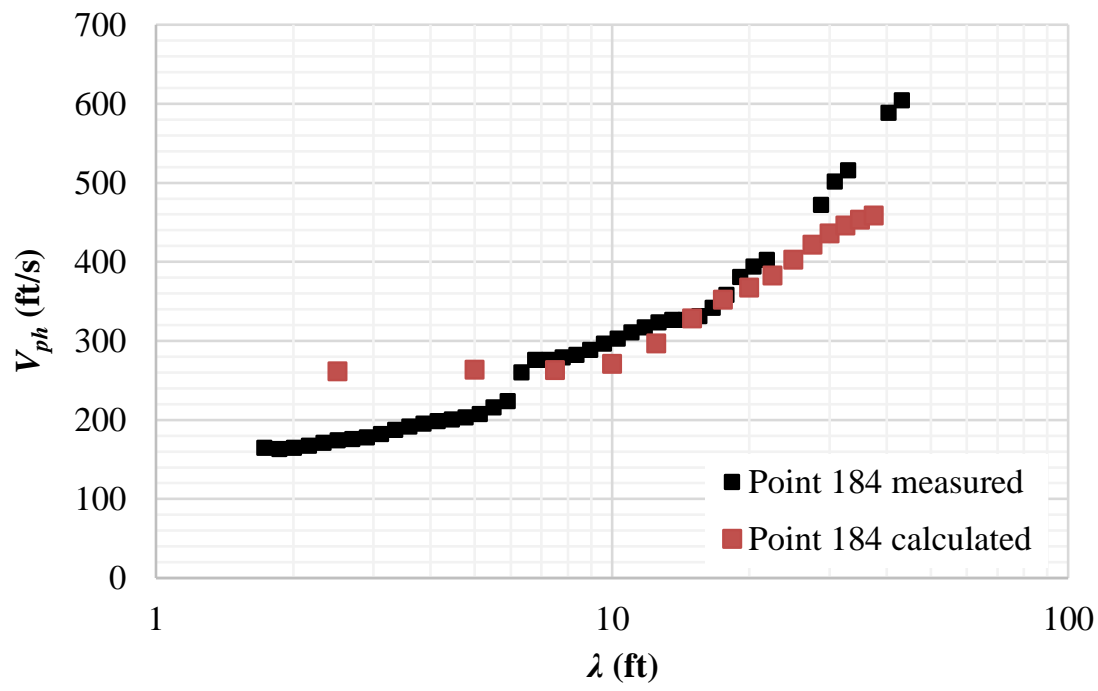

Figure C.22 - Point 184 measured vs calculated dispersion curves. 


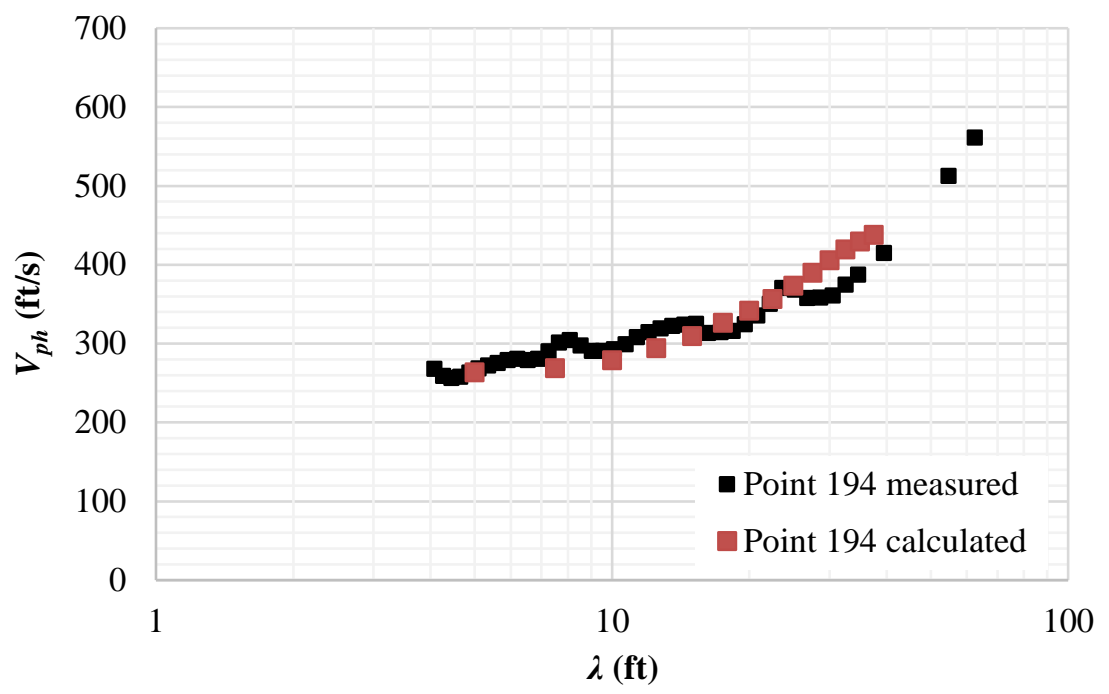

Figure C.23 - Point 194 measured vs calculated dispersion curves.

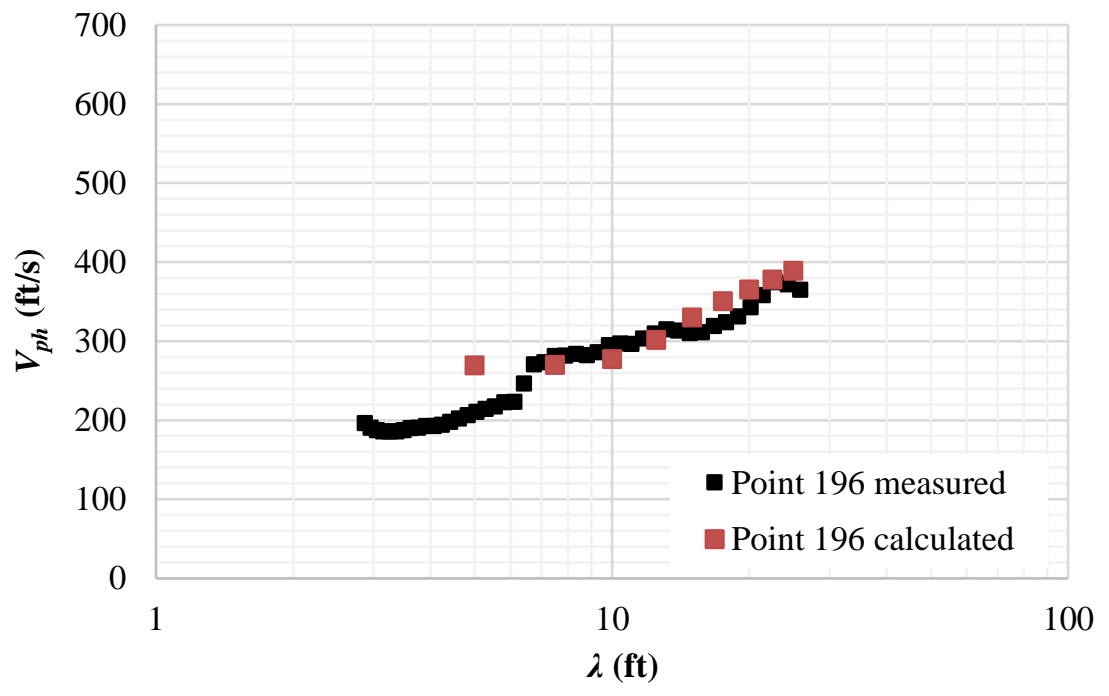

Figure C.24 - Point 196 measured vs calculated dispersion curves. 


\section{Appendix D: Sample Labadie UWL borings}

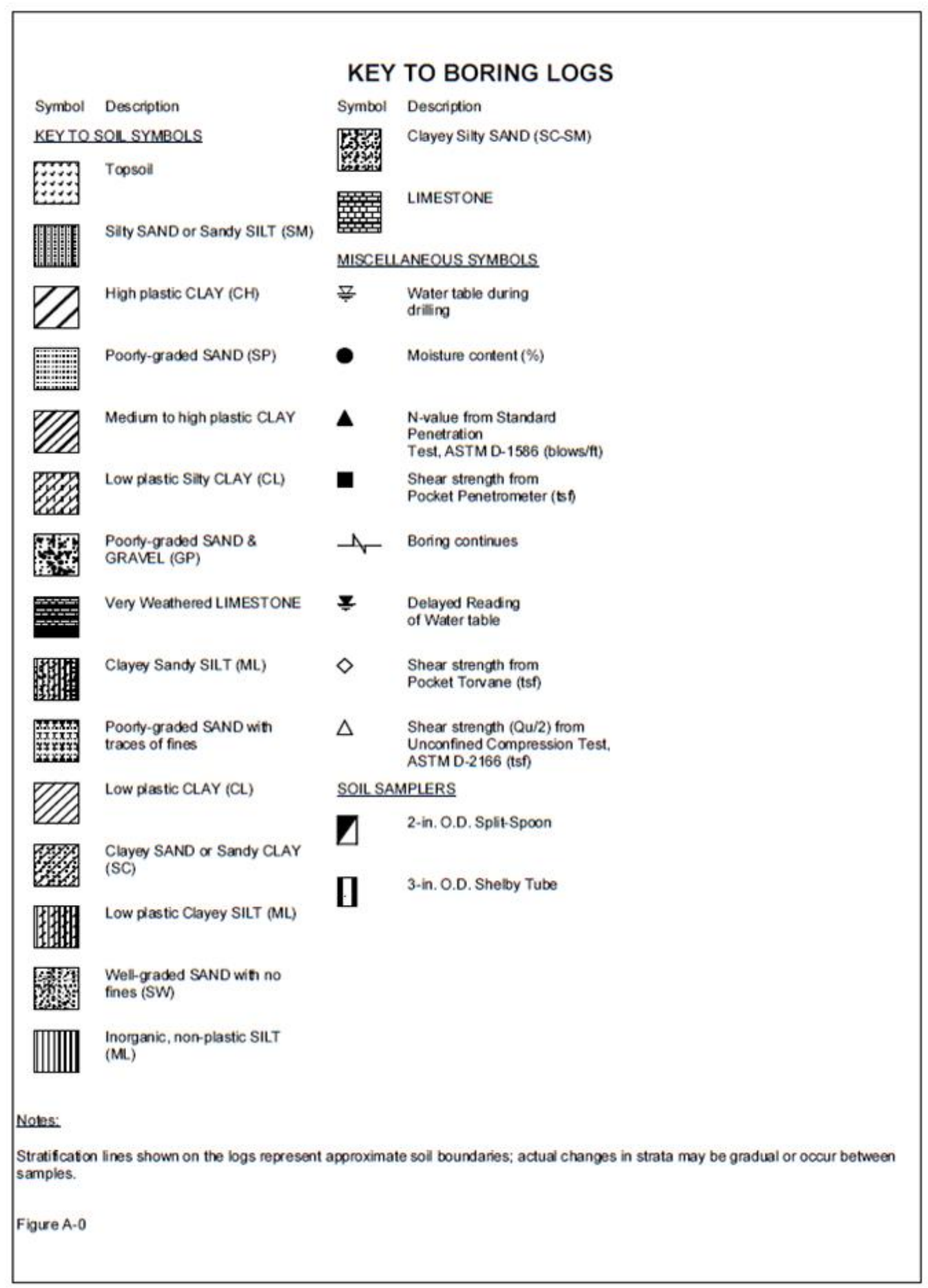

Figure D.1 - Boring key from GREDELL Engineering Resources, Inc. and Reitz \& Jens, Inc. 2011. 


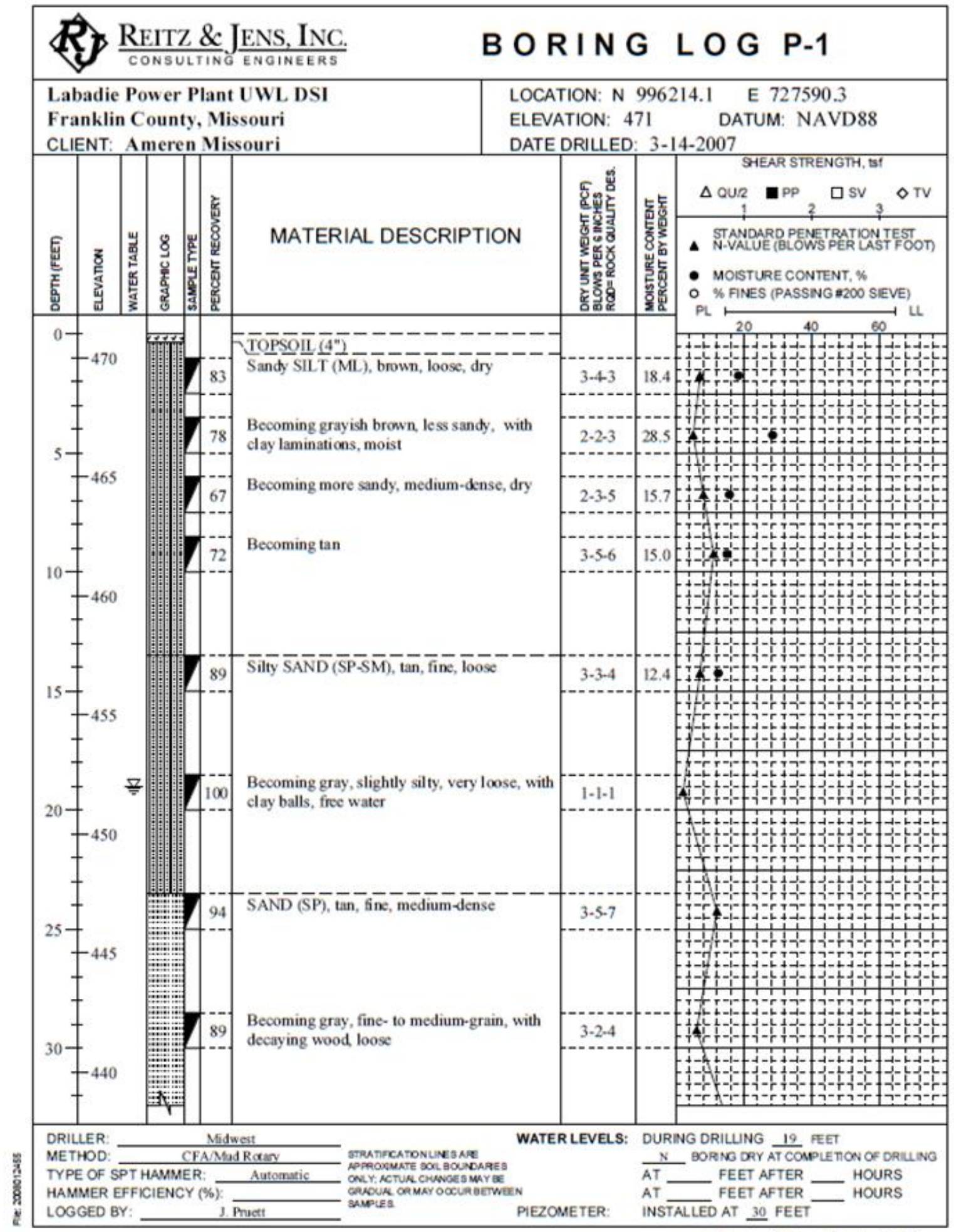

Figure A-1 Sheet 1 of 3

Figure D.2.1 - Boring P1 (approximately 130 yards north of Point 16) from GREDELL Engineering Resources, Inc. and Reitz \& Jens, Inc. 2011. 


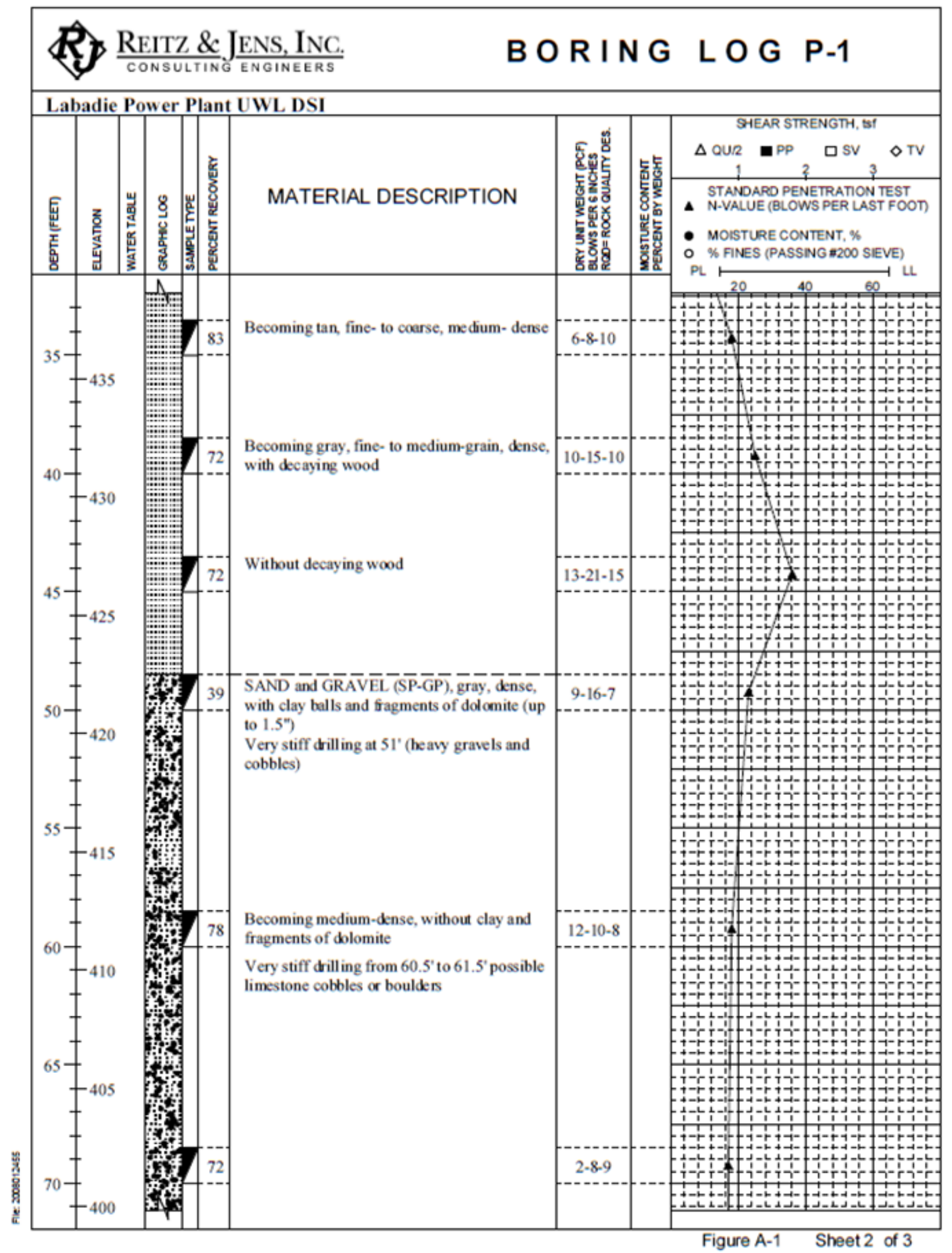

Figure D.2.2 - Boring P1 (approximately 130 yards north of Point 16) from GREDELL Engineering Resources, Inc. and Reitz \& Jens, Inc. 2011. 


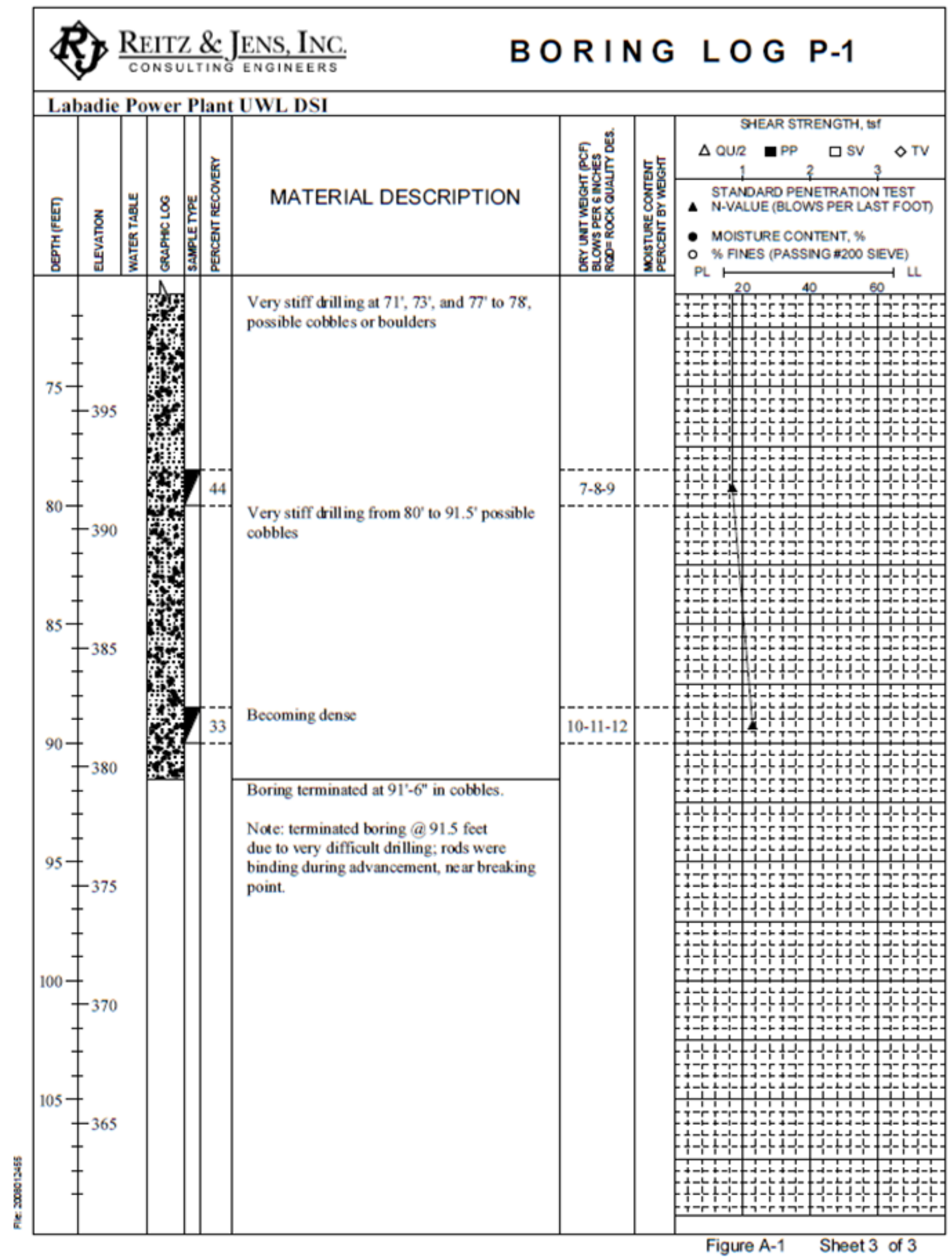

Figure D.2.3 - Boring P1 (approximately 130 yards north of Point 16) from GREDELL Engineering Resources, Inc. and Reitz \& Jens, Inc. 2011. 


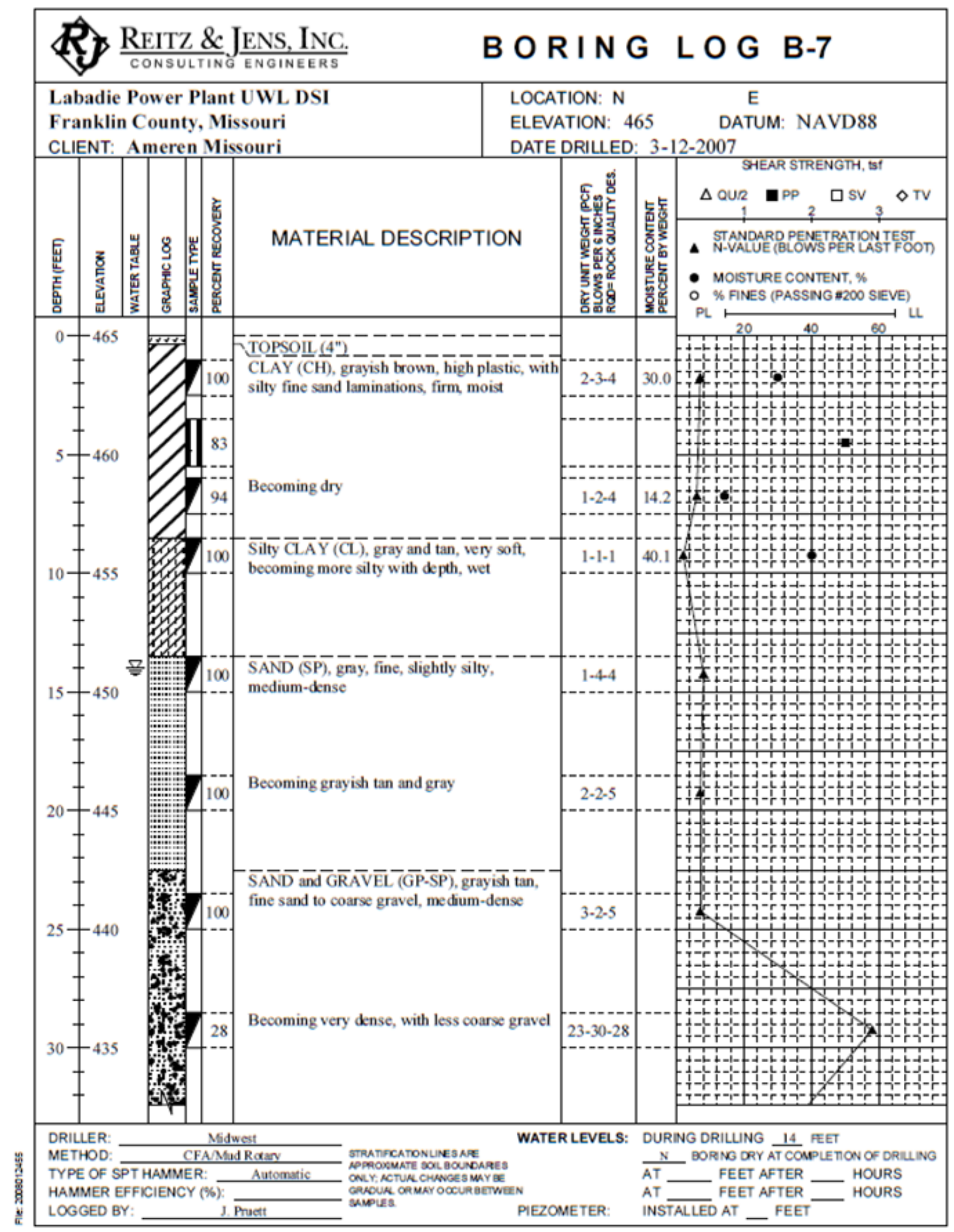

Figure A-7 Sheet 1 of 3

Figure D.3.1 - Boring B7 (approximately 200 yards northwest of Point 168) from GREDELL Engineering Resources, Inc. and Reitz \& Jens, Inc. 2011. 


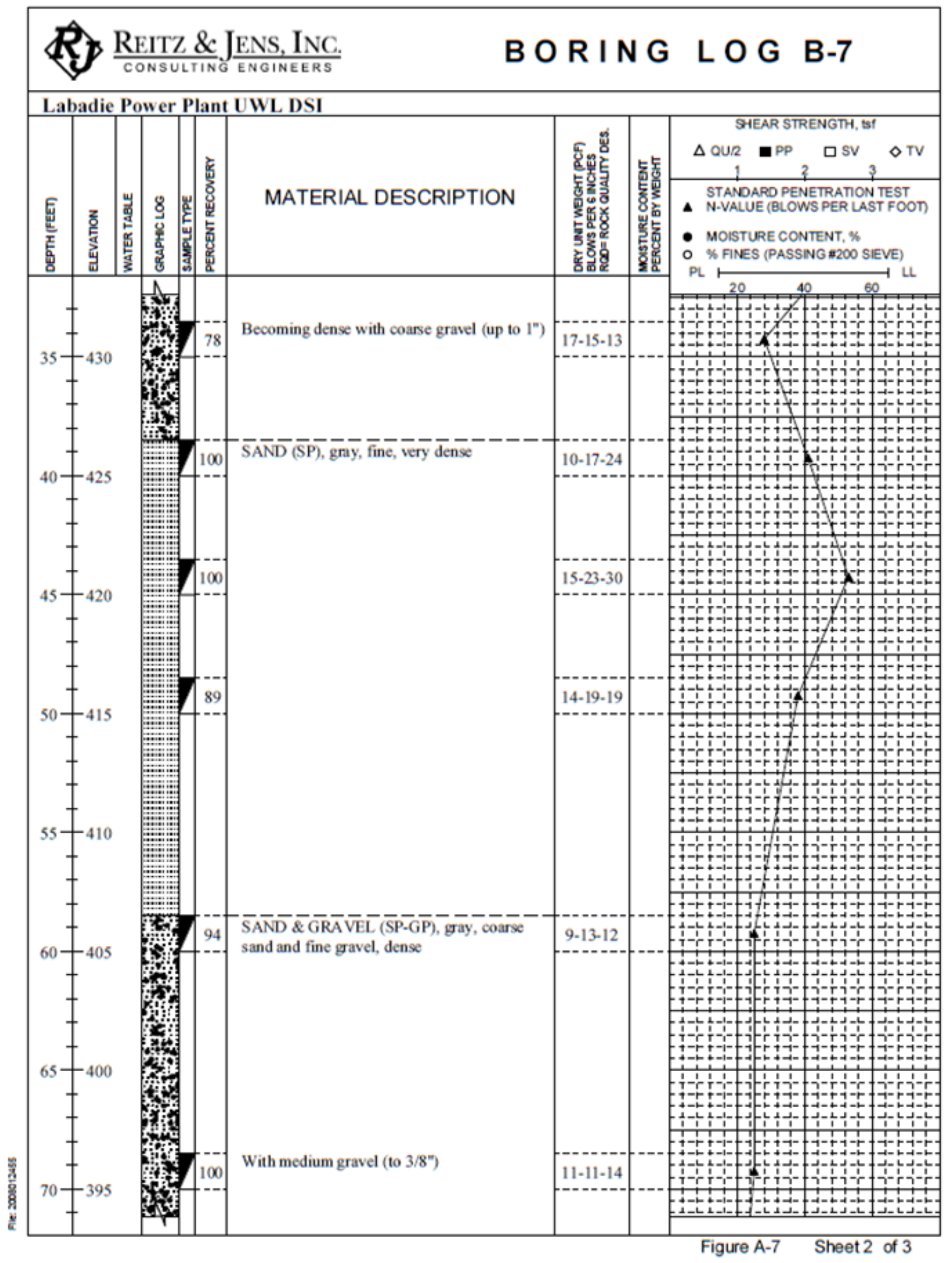

Figure D.3.2 - Boring B7 (approximately 200 yards northwest of Point 168) from GREDELL Engineering Resources, Inc. and Reitz \& Jens, Inc. 2011. 


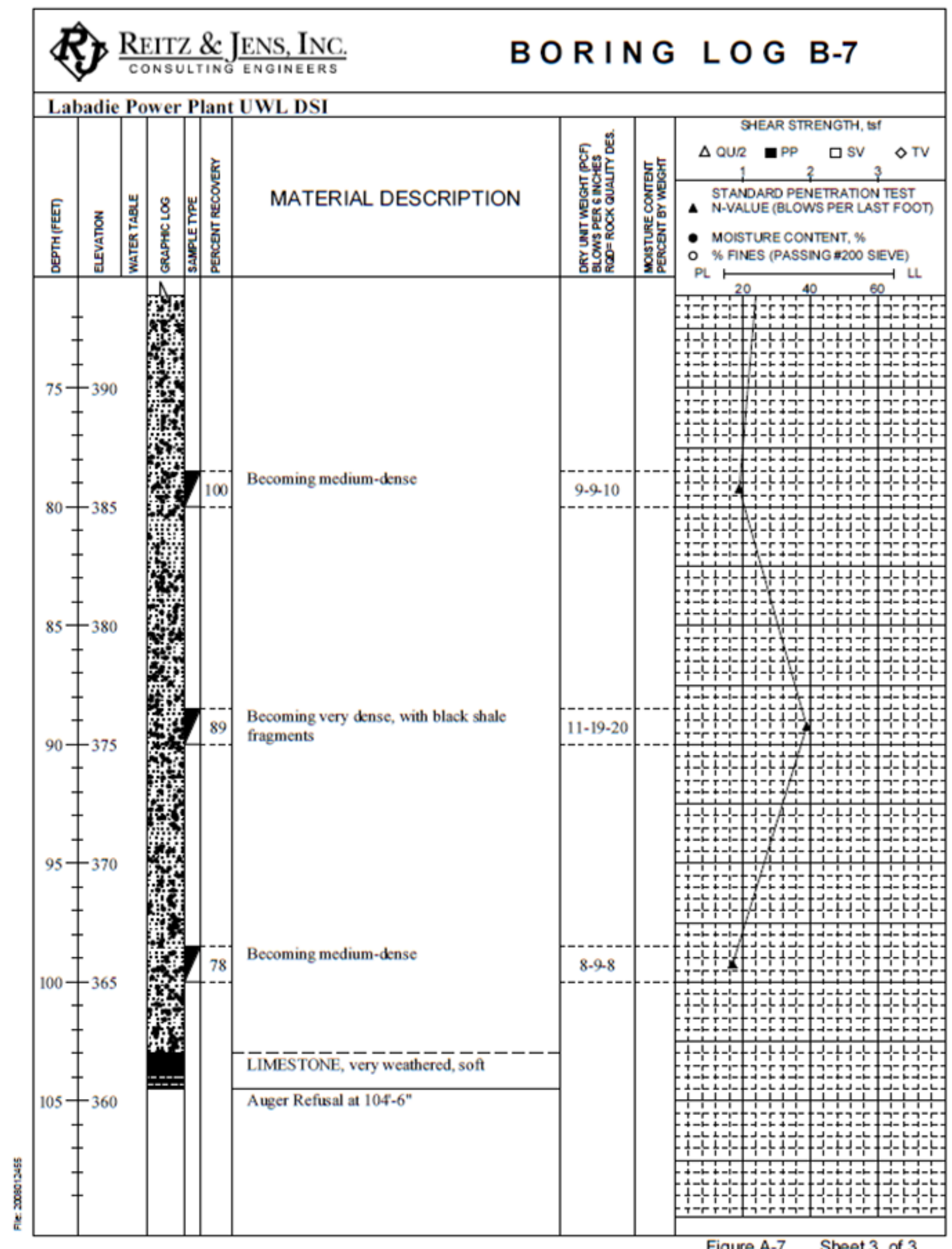

Figure A-7 Sheet 3 of 3

Figure D.3.3 - Boring B7 (approximately 200 yards northwest of Point 168) from GREDELL Engineering Resources, Inc. and Reitz \& Jens, Inc. 2011. 